\begin{tabular}{|l|l|}
\hline $\begin{array}{l}\text { 2. 10: (Receivin, Organization) } \\
\text { Distribution }\end{array}$ & $\begin{array}{l}\text { 3. From: (Originating Organization) } \\
\text { Production Planning \& Control }\end{array}$ \\
\hline 5. Proj./Prog./Dest./Div.: & $\begin{array}{l}\text { 6. Design Authority/ Design Agent/Cog. } \\
\text { Engr.: }\end{array}$ \\
Tank 241-S-109/Waste & \\
$\begin{array}{l}\text { Management/PPC/Analytical } \\
\text { Services }\end{array}$ & \\
\hline
\end{tabular}

8. Originator Remarks:

This document is being released into the supporting document system for retrievability purposes.

11. Receiver Remarks: 11A. Design Baseline Document? [] Yes [X] No

4. Related EDT No.:
N/A
7. Purchase Order No.:
N/A

$N / A$
For release.

9. Equip./Component No.: $N / A$

10. System/Bldg./Faeility: 241-5-109

12. Major Assm. Dug. No.: $N / A$

13. Permit/Permit Application No.: $N / A$

14. Required Response Date: $08 / 12 / 96$

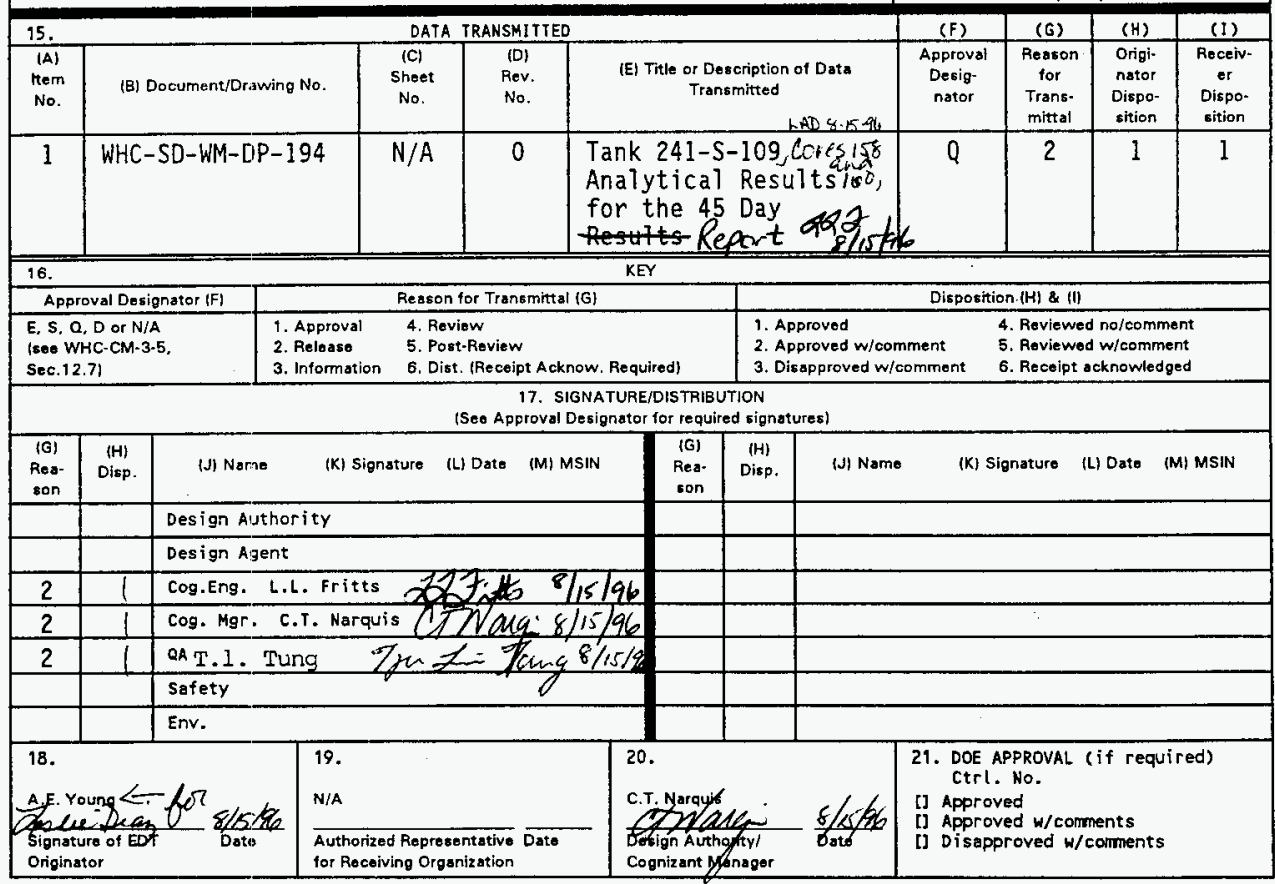




\section{Tank 241-S-109, Cores 158 and 160, Analytical Results for the 45 day Report}

\section{Larry L. Fritts}

West inghouse Hanford Company, Richland, WA 99352

U.S. Department of Energy Contract DE-AC06-87RL10930

EDT/ECN: EDT- 617492 UC: 2070

Org Code: $57670-7576 B$ Charge Code: MD378

B\&R Code: EW 3120074 Total Pages: 165

Key Words: Tank 241-S-109, Tank S-109, S-109, S Farm, 222-S Laboratory, 222-S, Analytical Results, Push Mode, Push, Core 158, Core 160

Abstract: $N / A$

TRADEMURK DISCLAIMER. Reference herein to any specific comercial product, process, or service by trade name, trademark, menufacturer, or otherwise, does not mecessarily constitute or imply its endorsment, recomendation, or favoring by the United States Government or any agency thereof or its contractors or subcontractors.

Printed in the United States of America. To obtain copies of this document, contact: WHC/BCS Document Control Services, P.0. Box 1970, Mailstop H6-08, Richland WA 99352, Phone (509) 372-2420; Fox $(509) 376-4989$.
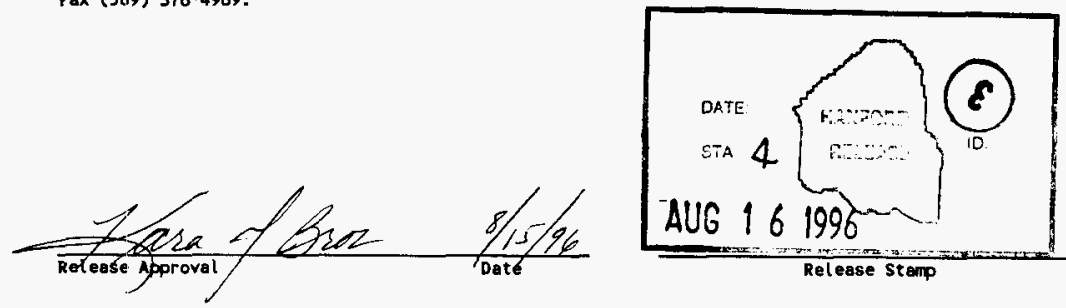

\section{Approved for Public Release}


P.O. Box 1970 Richland, WA 99352

WHC-SD-WM-DP-194, REV. 0

ANALYTICAL SERVICES

TANK 241-S-109

ANALYTICAL RESULTS

FOR THE 45 DAY REPORT

Project Coordinator: LARRY L. FRITTS

Prepared for the U.S. Department of Energy

Office of Environmental Restoration and Waste Management

by

Westinghouse Hanford Company

Box 1970

Richland, Washington 
WHC-SD-WM-DP-194, REV. 0

THIS PAGE WAS INTENTIONALLY LEFT BLANK 
WHC-SD-WM-DP-194, REV. 0

TABLE OF CONTENTS

Narrative ..................... 1

S-109 Sample Breakdown (Attachment I) . . . . . . . . . 13

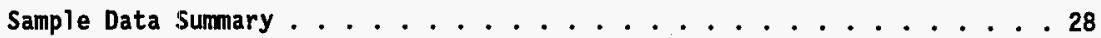

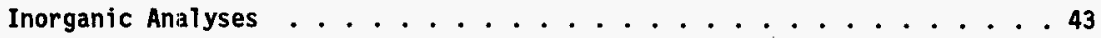

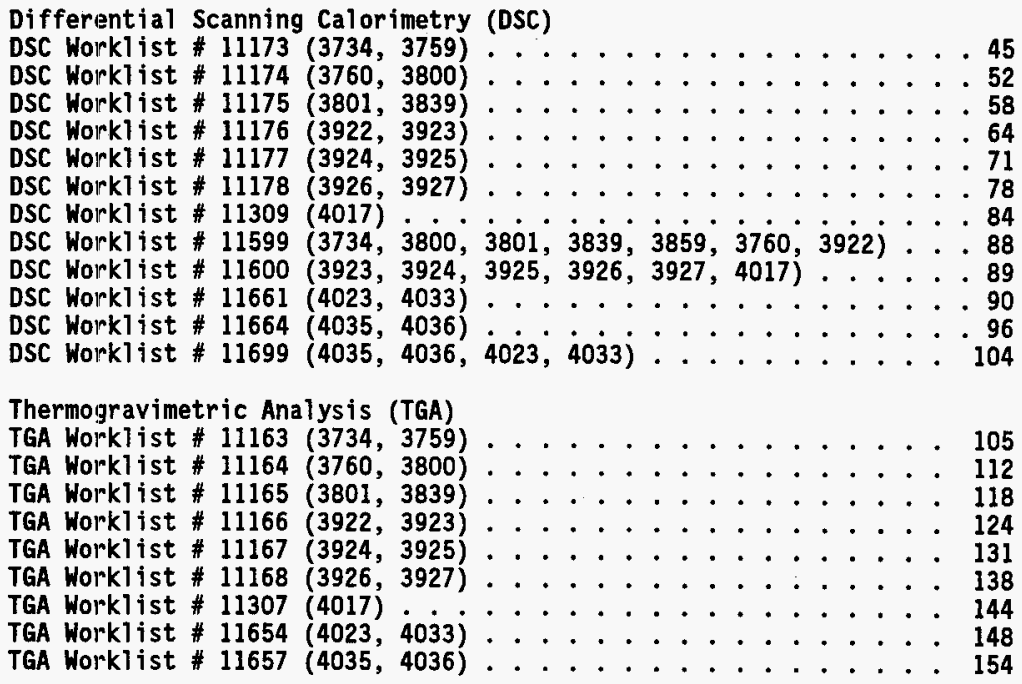

This document: consists of pages $1,2,11,12,13,14,28$, and 29 were intentionally left blank.

\section{TRADEMARKS:}

Perkin-Elmer is a Registered Trademark of Research and Manufacturing Company, Inc. Mettler is a Registered Trademark of Mettler Electronics 
WHC-SD-WM-DP-194, REV. 0

NARRATIVE 
WHC-SD-WM-DP-194, REV. 0

THIS PAGE WAS INTENTIONALLY LEFT BLANK 
WHC-SD-WM-DP-194, REV. 0

222-S ANALYTICAL SERVICES

TANK 241-S-109, CORES 158 AND 160,
ANALYTICAL RESULTS FOR THE 45 DAY REPORT

\section{Summary}

This document is the 45-day laboratory report for tank 241-S-109. Push mode core segments were removed from risers 14 and 16 between June 21, 1996, and July 3,1996. Segments were received and extruded at the 222-S Analytical Laboratory. Analyses were performed in accordance with the Tank 24l-S-109 Push Mode Core Sampling and Analysis Plan (TSAP) for this tank (Field, 1996) and the Safety Screening Data Quality Objective (DQO) (Dukelow, et al., 1995).

None of the subsamples submitted for Total Alpha Activity (AT) analysis or Differential Scanning Calorimetry (DSC) exceeded the action limits as stated in the Safety Screening Data Quality Objective (Dukelow, et al., 1995).

Primary safety screening results are included in the data summary table (Table 1). The raw data from DSC and TGA analyses are included in this report.

\section{Appearance and Sample Handling}

Two cores of twelve segments were expected from this tank. Sampling problems prevented the acquisition of complete cores.

Attachment 1 is a cross reference to relate the tank farm identification numbers to the 222-S Laboratory LabCore sample numbers. The subsamples generated in the laboratory for analysis are identified in these diagrams with their sources shown.

\section{Core 158}

Seven push mode core segments were removed from tank 241-S-109 riser 14 between June 21, 1996, and June 27, 1996. All segments were received by the 222-S Laboratory between July 11, 1996, and July 16, 1996. Table 2 summarizes the extrusion information.

\section{Core 160}

Five push mode core segments were removed from tank 241-S-109 riser 16 between July 2 , 1996, and July 3, 1996. All segments were received by the 222-S Laboratory between July 9 , 1996, and July 12, 1996. Table 3 summarizes the extrusion information. 
WHC-SD-WM-DP-194, REV. 0

\section{Field Blank}

A field blank was provided to the 222-S Laboratory with core 160 . This sample was treated as a drainable liquid in accordance with the TSAP.

\section{Liner Liquid}

The liner liquid recovered during extrusions will be analyzed by Inductively Coupled Plasma (ICP) spectroscopy and Ion Chromatography (IC) only. The results will be reported in a revision to this document. 
WHC-SD-WM-DP-194, REV. 0

Table 2. Sample Receipt and Extrusion Information for S-109, Core 158.

\begin{tabular}{|c|c|c|c|c|c|c|c|c|}
\hline $\begin{array}{l}\text { Qustumer } \\
\text { Id }\end{array}$ & Seginent & Sampled & Ractived & $\begin{array}{l}\text { Date } \\
\text { Dxurutidi }\end{array}$ & $\begin{array}{l}\text { Inthes } \\
\text { intridefe }\end{array}$ & $\begin{array}{l}\text { Liquid } \\
\text { necovered }(\mathrm{B})\end{array}$ & $\begin{array}{l}\text { Sothds } \\
\text { Acoovered }(g) \\
\end{array}$ & 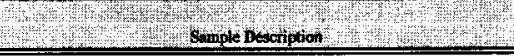 \\
\hline $96-362$ & 1 & $6 / 21 / 96$ & $7 / 11 / 96$ & $7 / 19 / 96$ & 8.0 & 0.0 & $\begin{array}{l}\text { 120.3--upper half } \\
57.5 \text { - lowet half }\end{array}$ & $\begin{array}{l}\text { The upper half solids were gray and resembled a moist crumbly salicake. } \\
\text { The lower half solids were yeliow and resembled a moist crumbly } \\
\text { saltcake. }\end{array}$ \\
\hline 96.363 & 2 & $6 / 24 / 96$ & $7 / 11 / 96$ & $7 / 16 / 96$ & 3.0 & 60.8--liner & 38.9.. Jower half & $\begin{array}{l}\text { The solids were yellow and resembled a dry crumbly saltcake. The liner } \\
\text { liquid was dark gray and opaque. }\end{array}$ \\
\hline $96.363 \mathrm{~A}$ & $2 \mathrm{~A}$ & $6 / 24 / 96$ & $7 / 11 / 96$ & $7 / 22 / 96$ & 2.0 & 0.0 & 23.9--lower half & $\begin{array}{l}\text { The solids were light yellow with small gray pieces and resembled a dry } \\
\text { saltcake. }\end{array}$ \\
\hline $96-363 \mathrm{~B}$ & 2B & $6 / 24 / 96$ & $7 / 11 / 96$ & $7 / 16 / 96$ & 5.0 & 0.0 & $\begin{array}{l}\text { 43.6--upper half } \\
\text { 20.9--lower half }\end{array}$ & $\begin{array}{l}\text { The upper half solids were bluish-gray and resembled a moist saltcake. } \\
\text { The lower half solids were light yellow and resembled a moist saltcake. }\end{array}$ \\
\hline $96-364$ & 3 & $6 / 29 / 96$ & $7 / 11 / 96$ & $7 / 19 / 96$ & 5.0 & 116.2-liner & $\begin{array}{l}\text { 77.5-upper half } \\
\text { 51.1-1ower half }\end{array}$ & $\begin{array}{l}\text { The upper half solids were gray-white and resembled a dry saltcake. The } \\
\text { lower half solids were yellow and resembled a dry saltcake. The liner } \\
\text { liquid was light gray and opaque. }\end{array}$ \\
\hline $96-364 A$ & $3 \mathrm{~A}$ & $6 / 26 / 96$ & $7 / 16 / 96$ & $7 / 19 / 96$ & 5.0 & 0.0 & 107.2--iower half & $\begin{array}{l}\text { The solids were yellow with a light gray tint on the upper end and } \\
\text { resembled a dry crumbly salicake. }\end{array}$ \\
\hline 96-365 & 4 & $6 / 27 / 96$ & $7 / 16 / 96$ & 7/19/96 & 5.0 & 0.0 & 80.9- lower half & The solids were light gray-white and resembled a flaky, moist saltcake. \\
\hline
\end{tabular}




\section{WHC-SD-WM-DP-194, REV. 0}

Table 3. Sample Receipt and Extrusion Information for S-109, Core 160.

\begin{tabular}{|c|c|c|c|c|c|c|c|c|}
\hline $\begin{array}{l}\text { Cuspond } \\
\text { Ild }\end{array}$ & Segment & somplod & Necelved & Date & $\begin{array}{l}\text { Inches } \\
\text { Extrudied }\end{array}$ & Lecovered $(\mathrm{g})$ & $\begin{array}{l}\text { solids } \\
\text { Recovered }(\mathrm{g})\end{array}$ & $\begin{array}{llll} & & & \\
\end{array}$ \\
\hline Field Blank & $\begin{array}{l}\text { Field } \\
\text { Blank }\end{array}$ & $7 / 8 / 96$ & $7 / 12 / 96$ & $7 / 22 / 96$ & 0.0 & 242.9--drainable & 0.0 & The drainable liquid was clear and colorless. \\
\hline $96-378$ & 1 & $7 / 2 / 96$ & $7 / 9 / 96$ & $7 / 16 / 96$ & 6.0 & 0.0 & 61.7-lower half & The solids were gray-white and resembled a dry saltcake. \\
\hline 96.379 & 2 & $7 / 2 / 96$ & $7 / 9 / 96$ & $7 / 16 / 96$ & 6.0 & 0.0 & 77,0 --lower half & $\begin{array}{l}\text { The solids were light yellow with a small amount of light greenish-gray } \\
\text { crystals and resembled a dry saltcake. }\end{array}$ \\
\hline $96-379 \mathrm{~A}$ & $2 \mathrm{~A}$ & $7 / 2 / 96$ & 7/9/96 & $7 / 16 / 96$ & 1.0 & 15.0--liner & 6.0--lower half & $\begin{array}{l}\text { The lower half solids were light yellow with a slight green tint and } \\
\text { resembled a fine, crumbly, dry saltcake. The liner liquid was dark gray } \\
\text { and opaque. }\end{array}$ \\
\hline 96-379-B & 2B & $7 / 2 / 96$ & $7 / 11 / 96$ & $7 / 22 / 96$ & 3.5 & 125.2-liner & $\begin{array}{l}\text { 30.0--upper half } \\
32.5 \text {--lower half }\end{array}$ & $\begin{array}{l}\text { The liner liquid had a slight yellow color and was opaque. The upper half } \\
\text { solids were yellow with a gray tint and resembled a dry, crumbly saltcake } \\
\text { The lower half solids were gray and resembled a dry, crumbly saltcake. }\end{array}$ \\
\hline $96-379 \mathrm{C}$ & $2 \mathrm{C}$ & $7 / 3 / 96$ & $7 / 12 / 96$ & $7 / 22 / 96$ & Unmeasurable & 68.8--drainable & Unmeasurable & $\begin{array}{l}\text { The drainable liquid was green and opaque. The solids were gray and } \\
\text { resembled a wet salt. Separation of solids and liquids was not possible. } \\
\text { All of the sample was subsampled as drainable liquid. The filtered solids } \\
\text { will be archived. }\end{array}$ \\
\hline
\end{tabular}

Approximate inches Extruded 


\section{WHC-SD-WM-DP-194, REV. 0}

\section{Analytical Results Summary}

The data summary table (Table 1) included in this report compiles the safety screening analytical results and applicable notification limits associated with each subsample submitted.

\section{Differential Scanning Calorimetry (DSC)}

None of the subsamples submitted for Differential Scanning Calorimetry (DSC) exceeded the action limits as stated in the TSAP.

The DSC analyses were performed in duplicate on direct subsamples. The exothermic energy based on dry weight of subsample was calculated for all subsamples. The average of the thermogravimetric analysis (TGA) results for each subsample was used in the dry weight correction for that subsample.

Relative percent differences (RPD) greater than $20 \%$ were reported for four of the sixteen subsamples. These were the result of small exotherms and sample inhomogeneity. The results for these subsamples were near the detection limit for the instrument; at these levels precision is compromised. No further analysis of these subsamples was requested.

The field blank average result was $0.00 \mathrm{~J} / \mathrm{g}$. Standard recovery criterion was met for all subsamples.

\section{Thermogravimetric Analysis (TGA)}

The TGA analyses were performed in duplicate on direct subsamples. Typically results were determined by summing weight loss steps below $200^{\circ} \mathrm{C}$; weight loss steps above this were not used to determine the result. More information may be obtained by examining the raw data.

RPDs greater than $20 \%$ were reported for five of the sixteen subsamples. These were the result of low water content and sample heterogeneity. The results for these subsamples were near the detection limit for the instrument; at these levels precision is compromised. No further analysis for these subsamples was requested. Low water content for six of the subsamples resulted in thermograms with a small scale. This small scale shows the vibrations of the balance and does not affect data quality. The cognizant chemist noted that the thermogram for sample S96T004033 (core 160, segment 2C, drainable liquid) showed several weight loss reactions occurring. All of these weight loss reactions were identified as the same peak.

The field blank average result was $99.92 \%$. Standard recovery criterion was met for all subsamples. 
WHC-SD-WM-DP-194, REV. 0

\section{Density}

Bulk density was run for eleven of the fifteen solid subsamples. Subsamples S96T004016 (core 158 , segment 2A, lower half), S96T003758 (core 158, segment 2B, lower half), and S96T003771 (core 160 , segment $2 \mathrm{~A}$, lower half) did not provide enough material for a bulk density determination. Sample S96T004029 (core 160, segment 2B, lower half) was too dry to perform a bulk density measurement.

The results of the bulk density test ranged from $1.19 \mathrm{~g} / \mathrm{mL}$ to $1.73 \mathrm{~g} / \mathrm{mL}$. The higher bulk density of $1.73 \mathrm{~g} / \mathrm{mL}$ was used to calculate the solid total alpha activity notification limit for the $\operatorname{tank}(35.5 \mu \mathrm{Ci} / \mathrm{g})$.

Total alpha results for liquids do not require correction for density. The specific gravity results for the liquid subsamples will be reported in a revision to this document.

\section{Total Alpha (AT)}

The liquid AT results were below the total alpha activity notification limit of $61.5 \mu \mathrm{Ci} / \mathrm{mL}$. All solid AT results were below the total alpha activity notification limit of $35.5 \mu \mathrm{Ci} / \mathrm{g}$ (based on a bulk density of $1.73 \mathrm{~g} / \mathrm{mL}$ ).

The AT analyses were performed in duplicate on direct subsamples for the liquids. Solid subsamples were prepared for analysis by performing a fusion digest in duplicate. The fusion digest is indicated with an " $F$ " in the aliquot class (A\#) column in the data summary table (Table 1).

An RPD greater than the precision as defined by the TSAP was reported for eight of the sixteen subsamples. Count replicate results and similar result behavior with the original analysis show this to be the result of low sample alpha activity and sample inhomogeneity. Low spike recoveries are the result of self-absorption by the solids left on the planchet after drying. The low alpha activity allowed a larger sample size to be used in the analysis; lowering the detection limit, but increasing the amount of residual solids due to the salt like nature of these subsamples. The results may be biased low by this process. Continuing reruns were not requested.

The field blank result was near the detection limit giving a sample result less than $6.61 \mathrm{e}-6 \mu \mathrm{Ci} / \mathrm{mL}$ and a duplicate result of $3.58 \mathrm{e}-6 \mu \mathrm{Ci} / \mathrm{mL}$. Standard recovery criterion was met for all subsamples. 


\section{Procedures}

Table 4 lists the analytical procedures used for performing the sample analyses. Abbreviations for analyses are defined in the table notes.

Table 4. Analytical Procedures

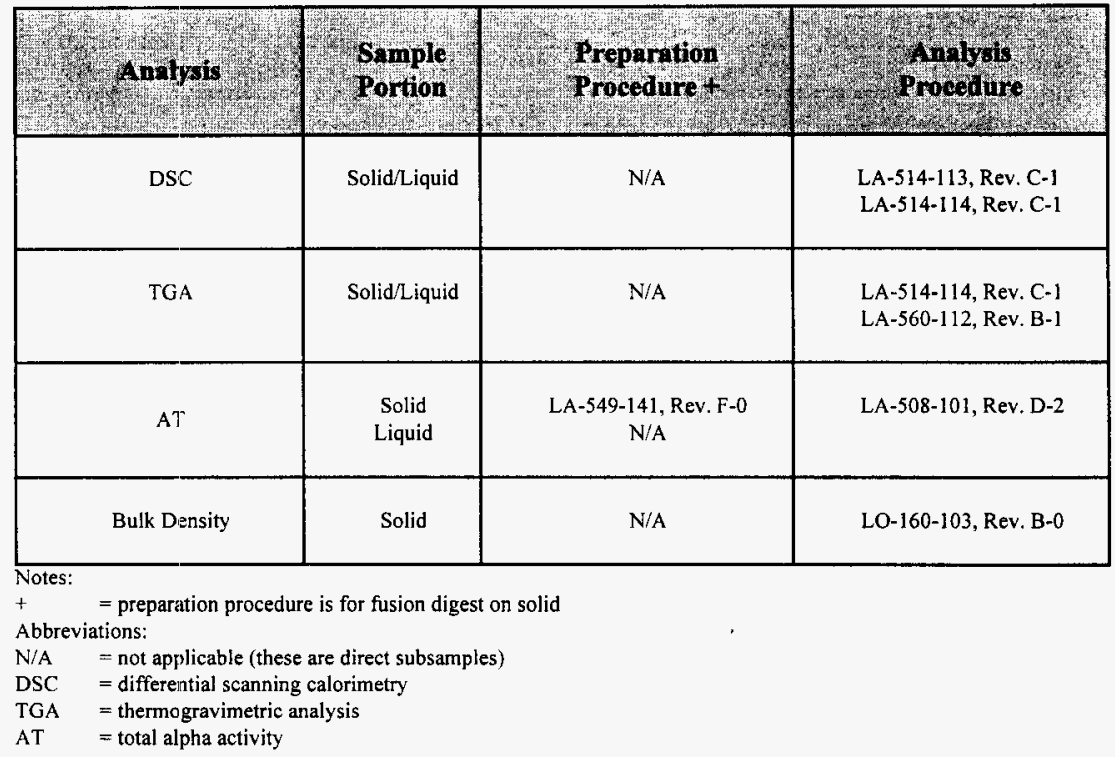




\section{WHC-SD-WM-DP-194, REV. 0}

\section{References}

Dukelow, G. T., J. W. Hunt, H. Babad, and J. E. Meacham, 1995, Tank Safety Screening Data Quality Objective, WHC-SD-WM-SP-004, Rev. 2, Westinghouse Hanford Company, Richland, WA 99352.

Field, J. G., 1996, Tank 24l-S-109 Push Mode Core Sampling and Analysis Plan, WHC-SD-WM-TSAP-087, Rev. 1, Westinghouse Hanford Company, Richland, WA 99352. 
WHC-SD-WM-DP-194, REV. 0

THIS PAGE WAS INTENTIONALLY LEFT BLANK 
WHC-SD-WM-DP-194, REV. 0

THIS PAGE WAS INTENTIONALLY LEFT BLANK 
WHC-SD-WM-DP-194, REV. 0

ATTACHMENT I

S-102 SAMPLE BREAKDOWN 
WHC-SD-WM-DP-194, REV. 0

THIS PAGE WAS INTENTIONALLY LEFT BLANK

14 
S-109

Core:160

Seg: FB (Field Blank)

S96T003694
Attachment 1 Page 1 of 13
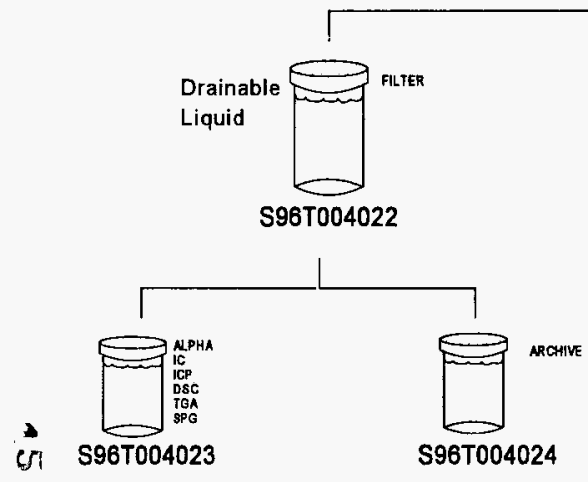

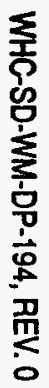




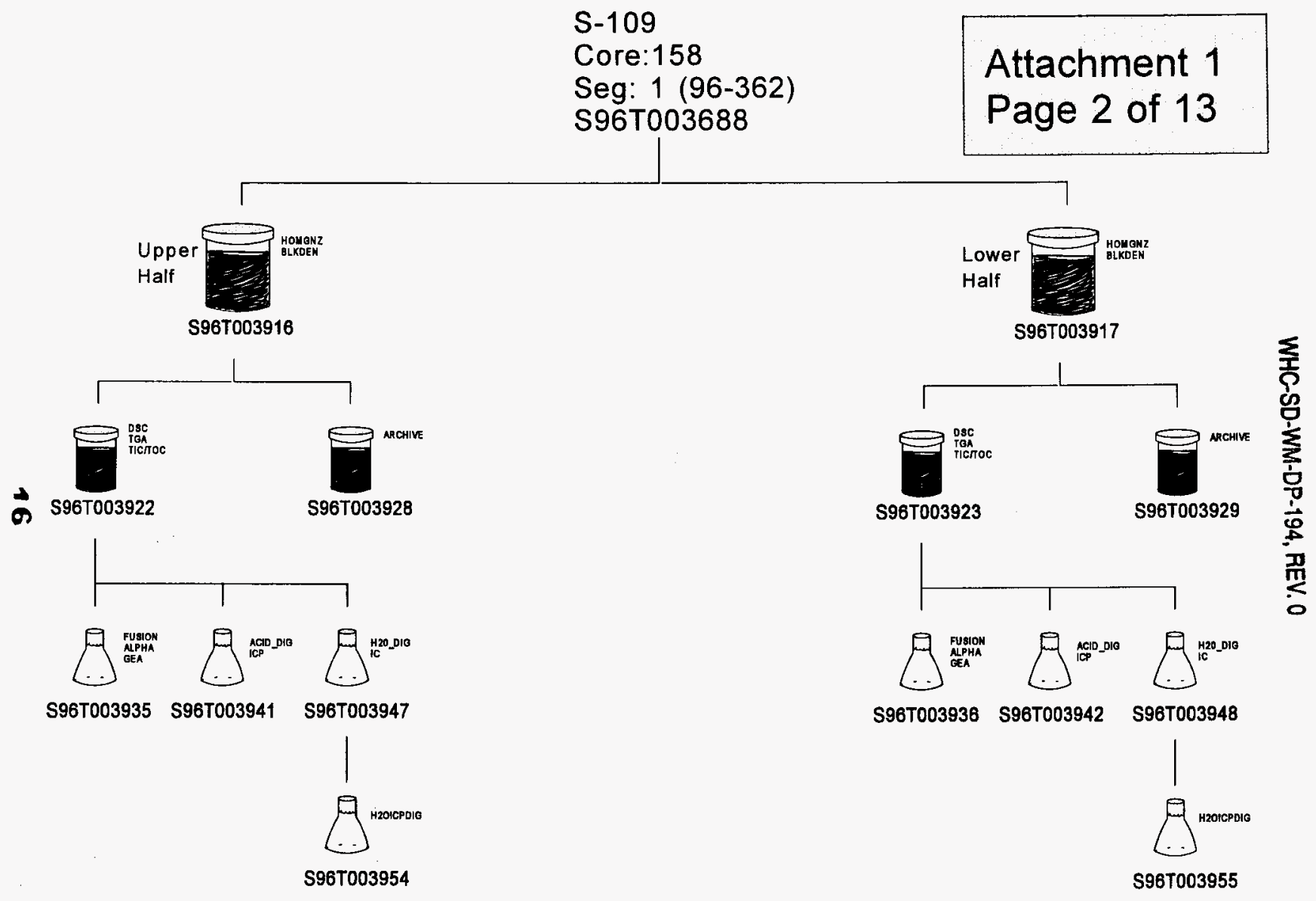




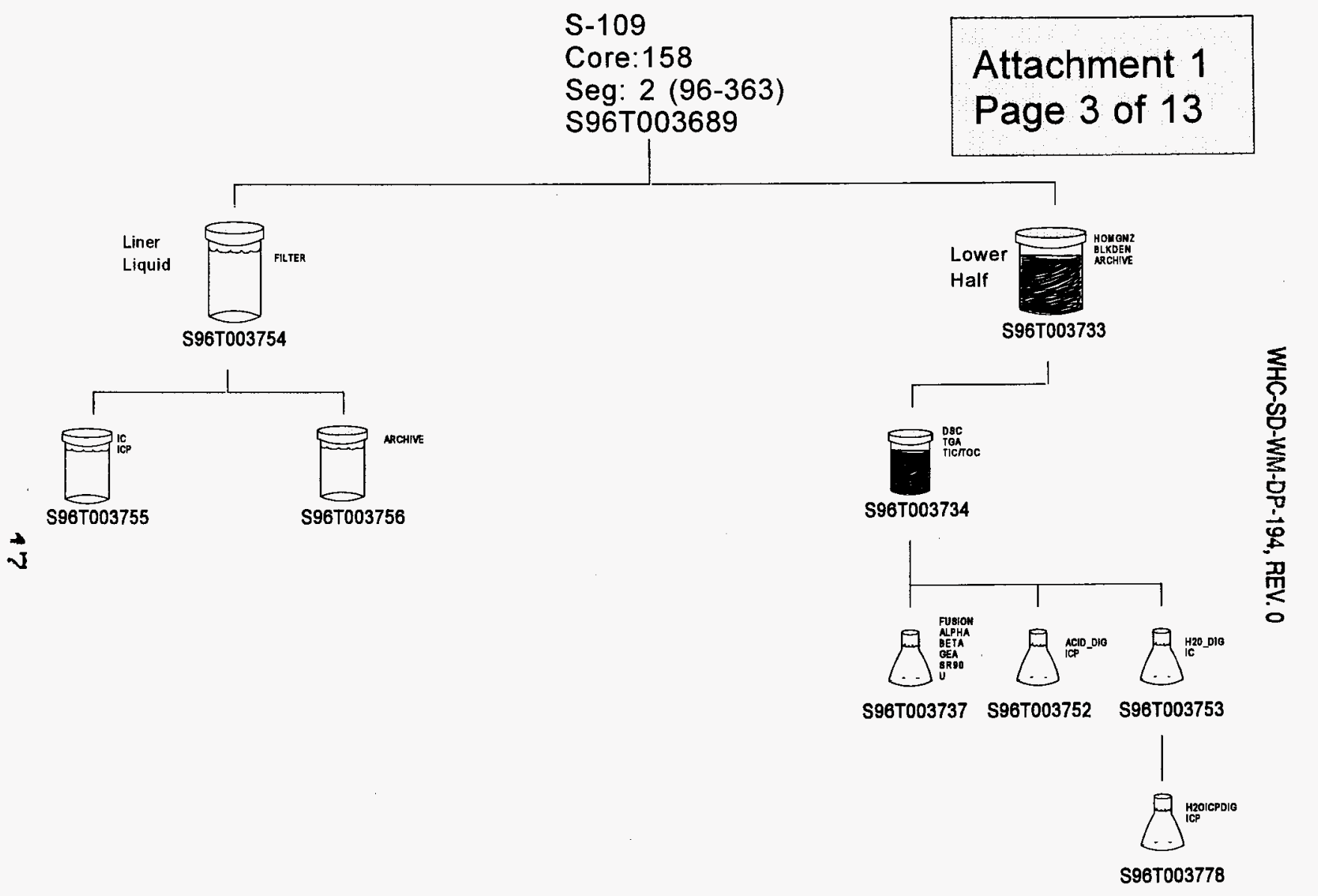




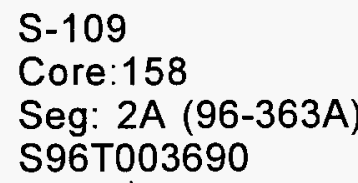

\section{Attachment 1 Page 4 of 13}

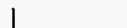

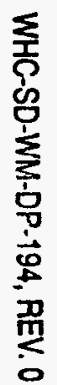

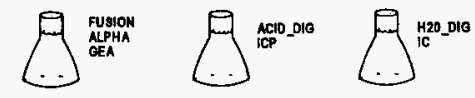

S96T004018 S96T004019

S96T004020

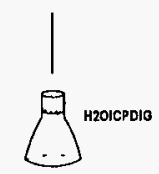

S96T004021 


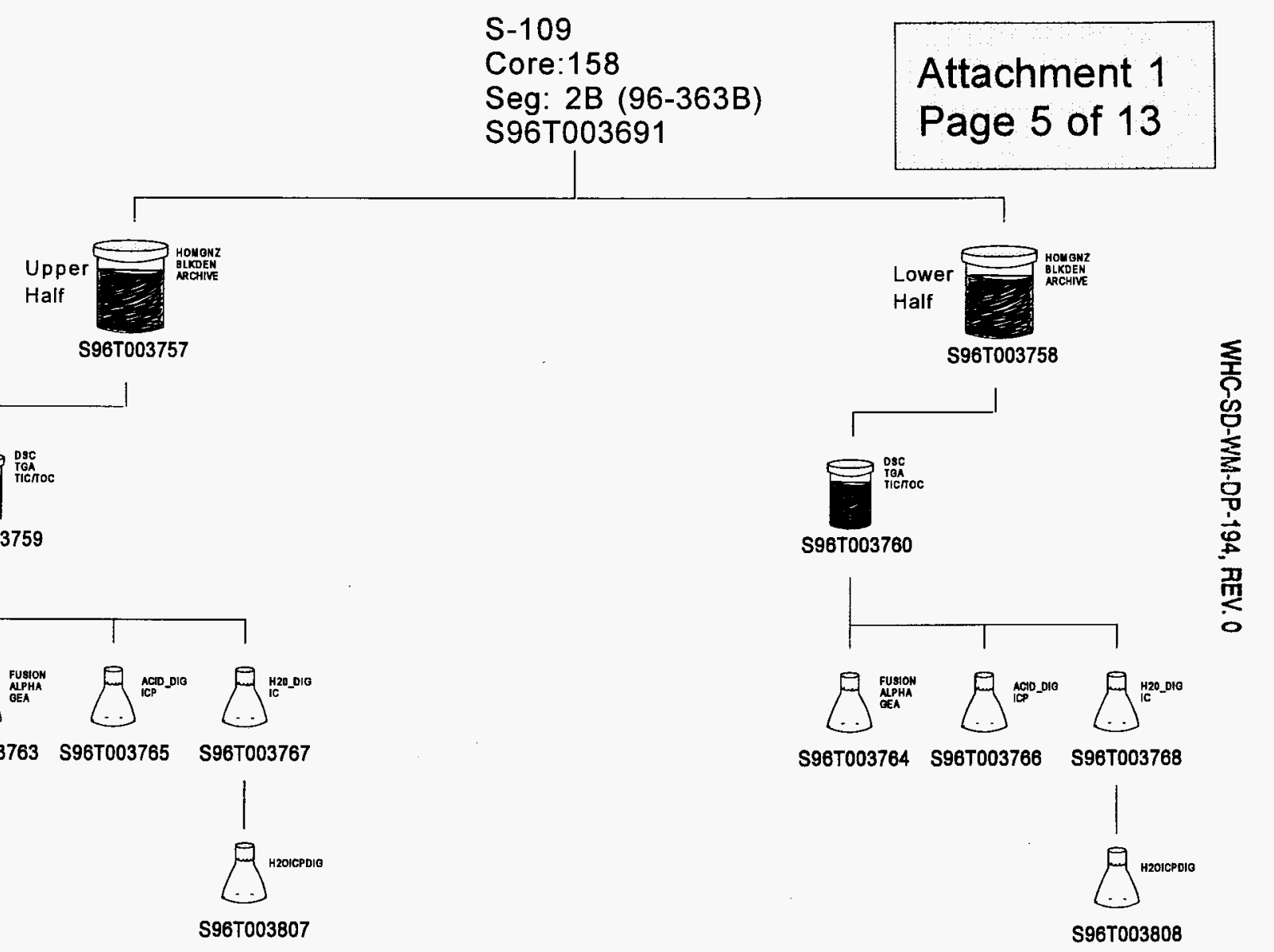




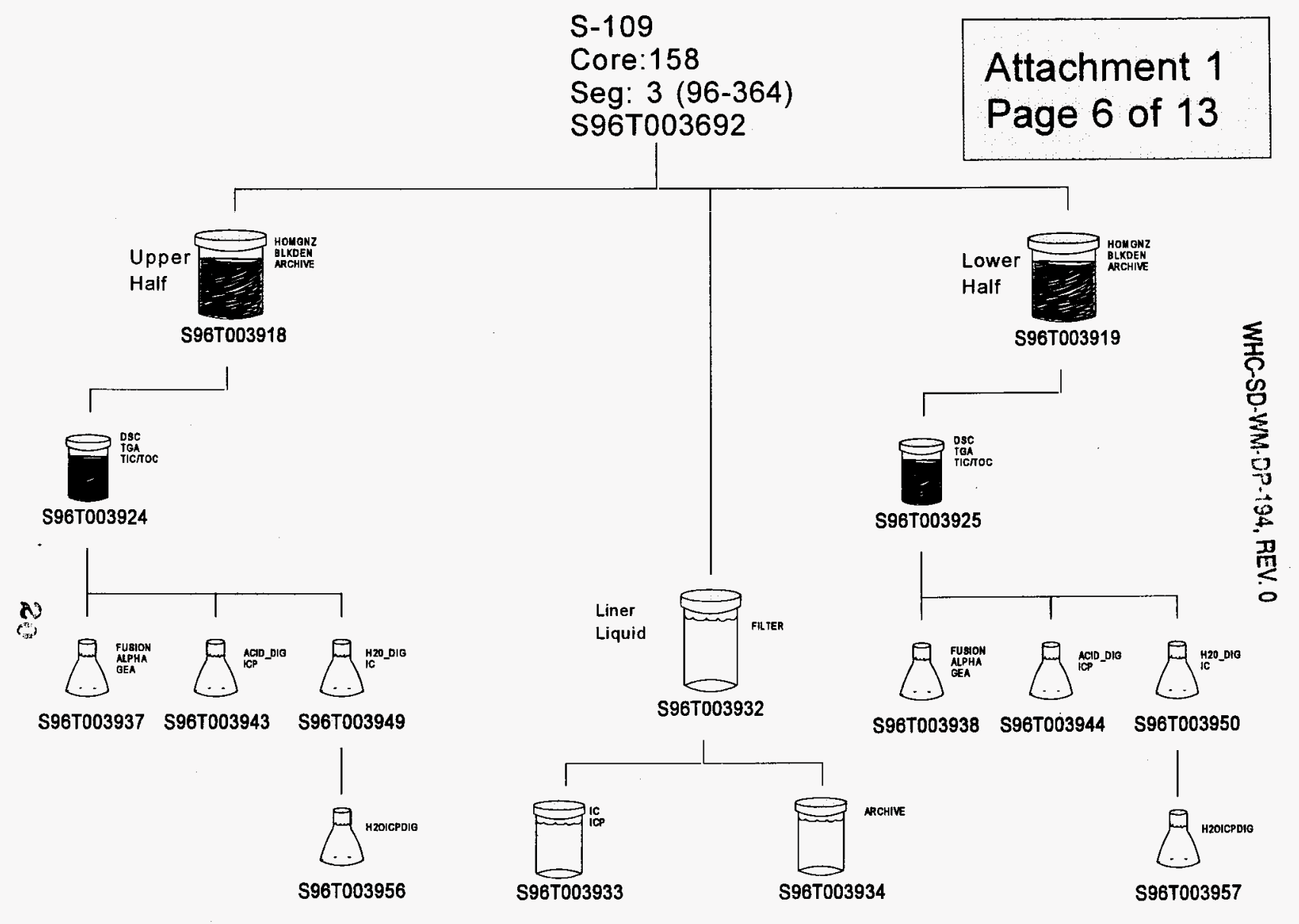




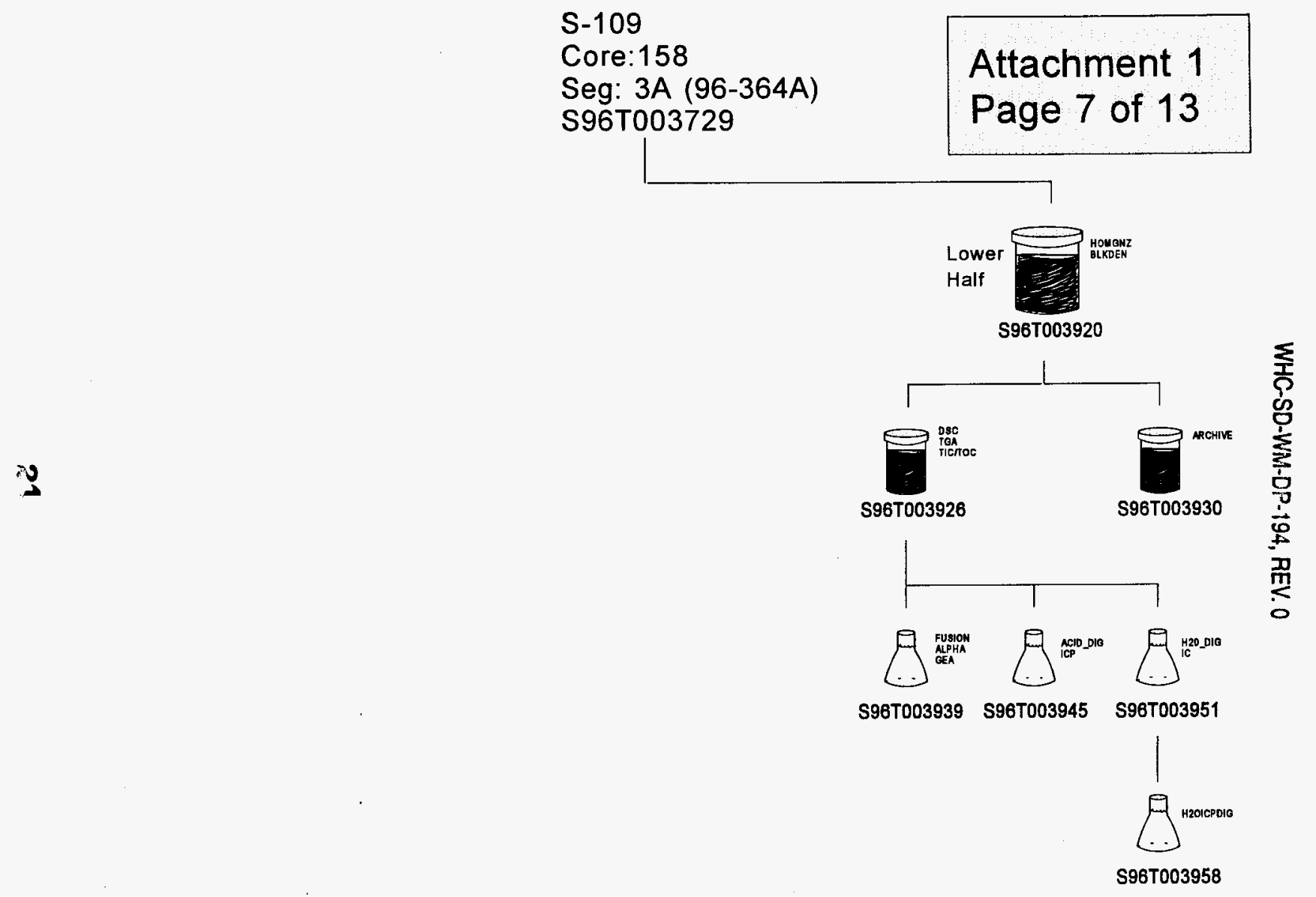


S-109

Core:158

Seg: 4 (96-365)

S96T003730

\section{Attachment 1 Page 8 of 13}

N

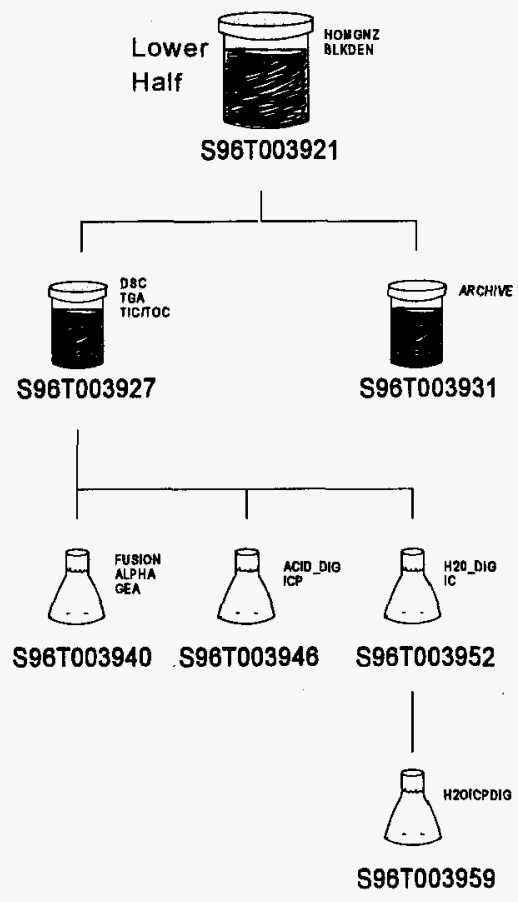

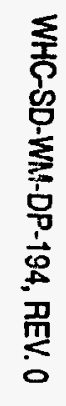


S-109

Core: 160

Seg: 1 (96-378)

S96T003671

\section{Attachment 1 Page 9 of 13}

2

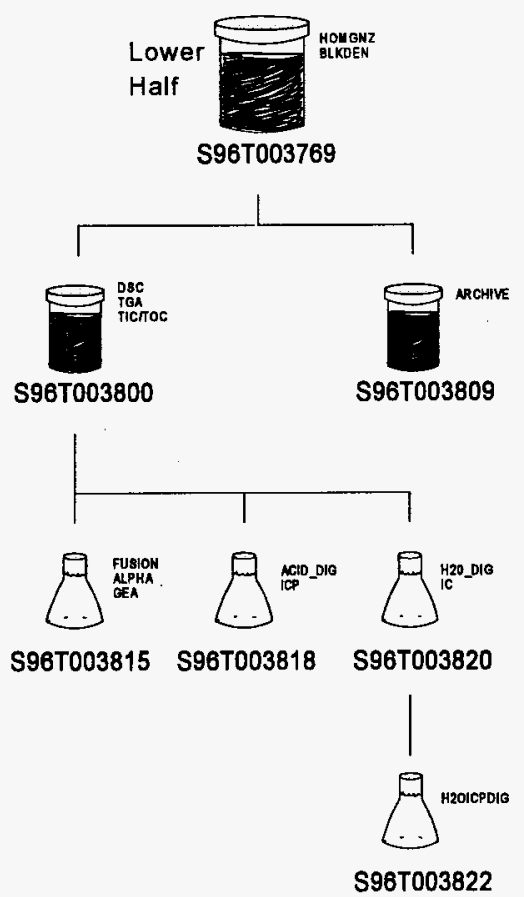


S-109

Core: 160

Seg: 2 (96-379)

S96T003672

\section{Attachment 1 Page 10 of 13}

$\omega$
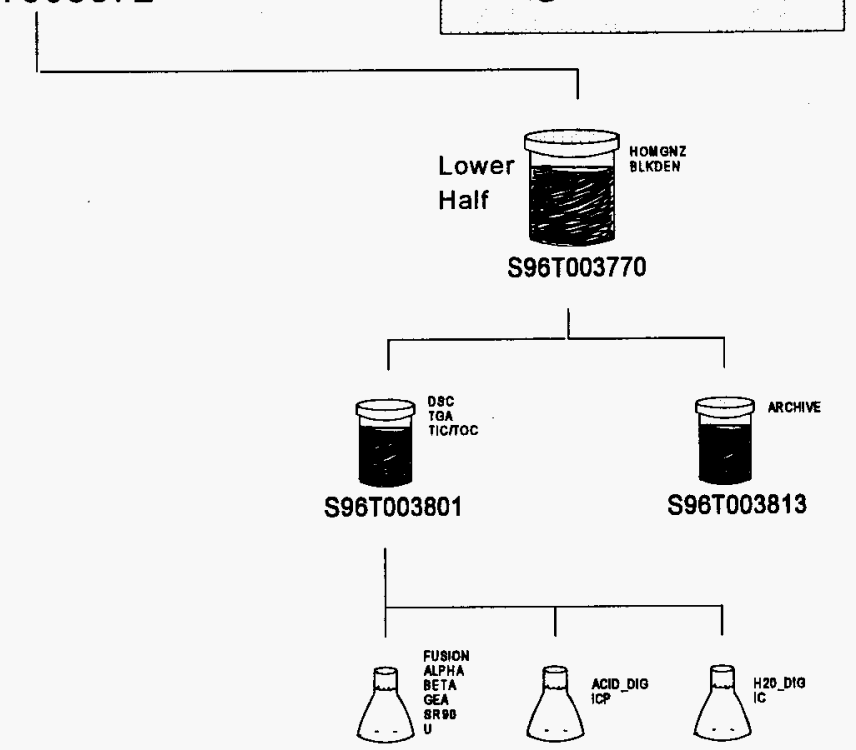

S96T003814

S96T003819

S96T003821

章

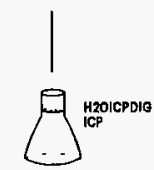

S96T003823 


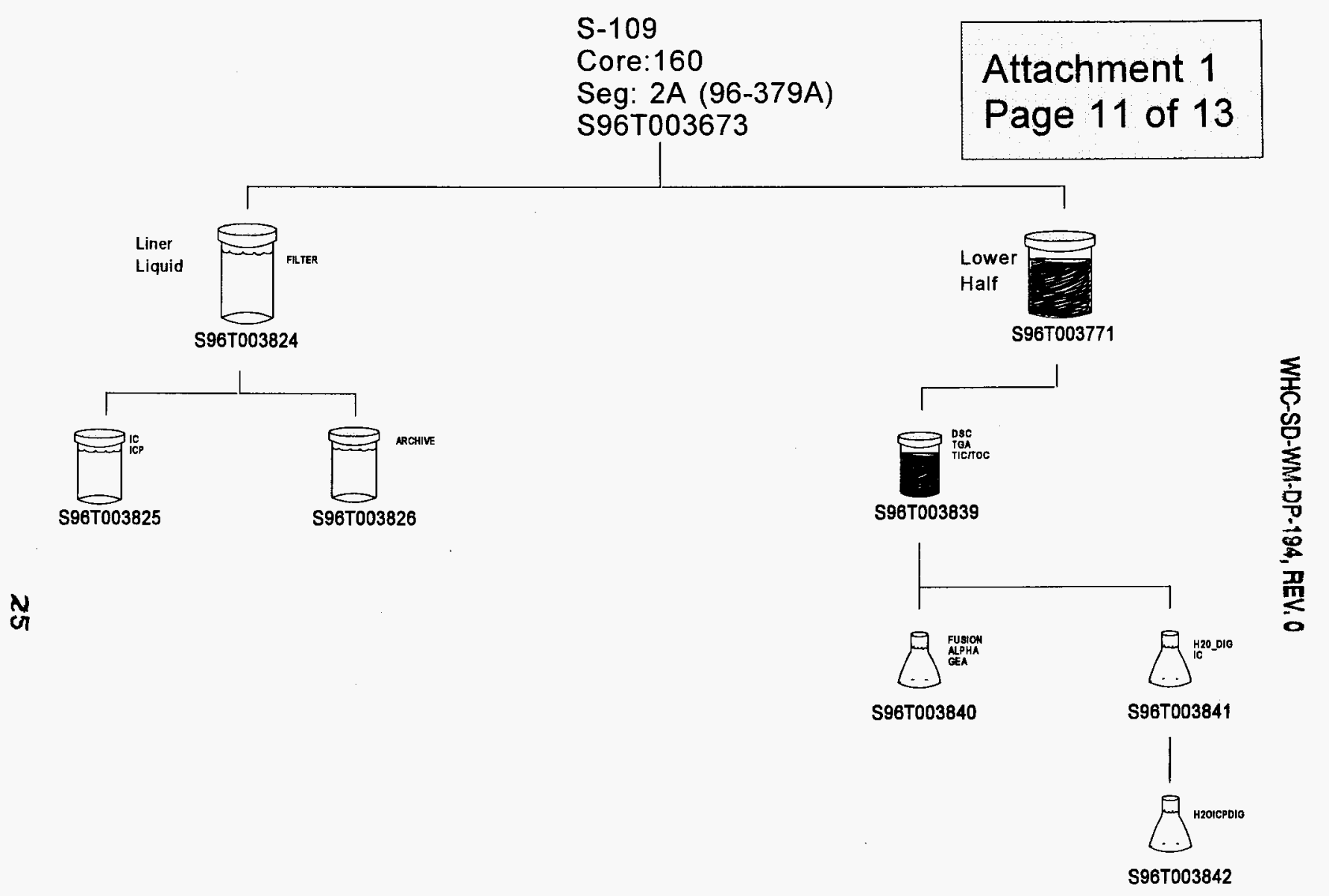




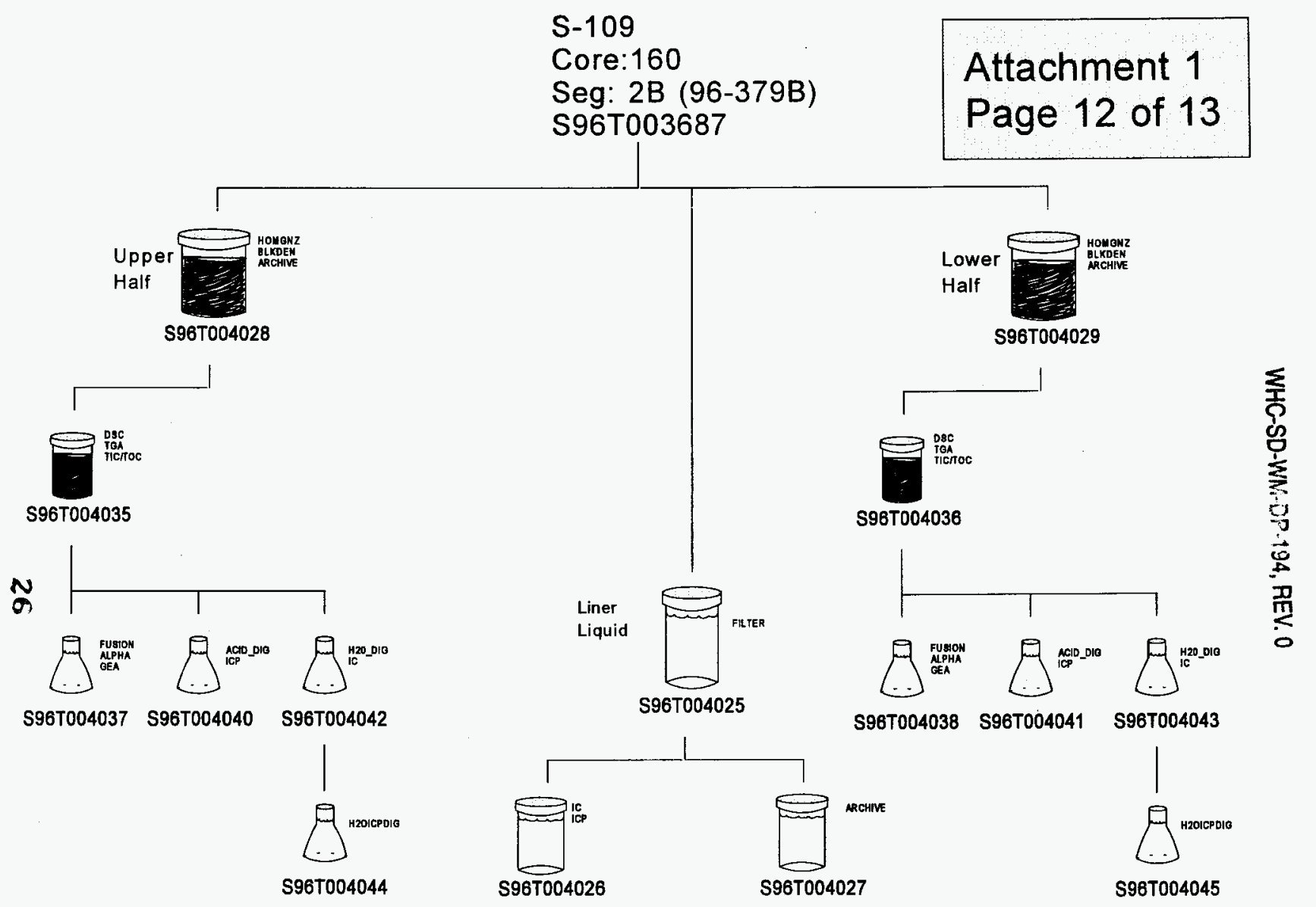




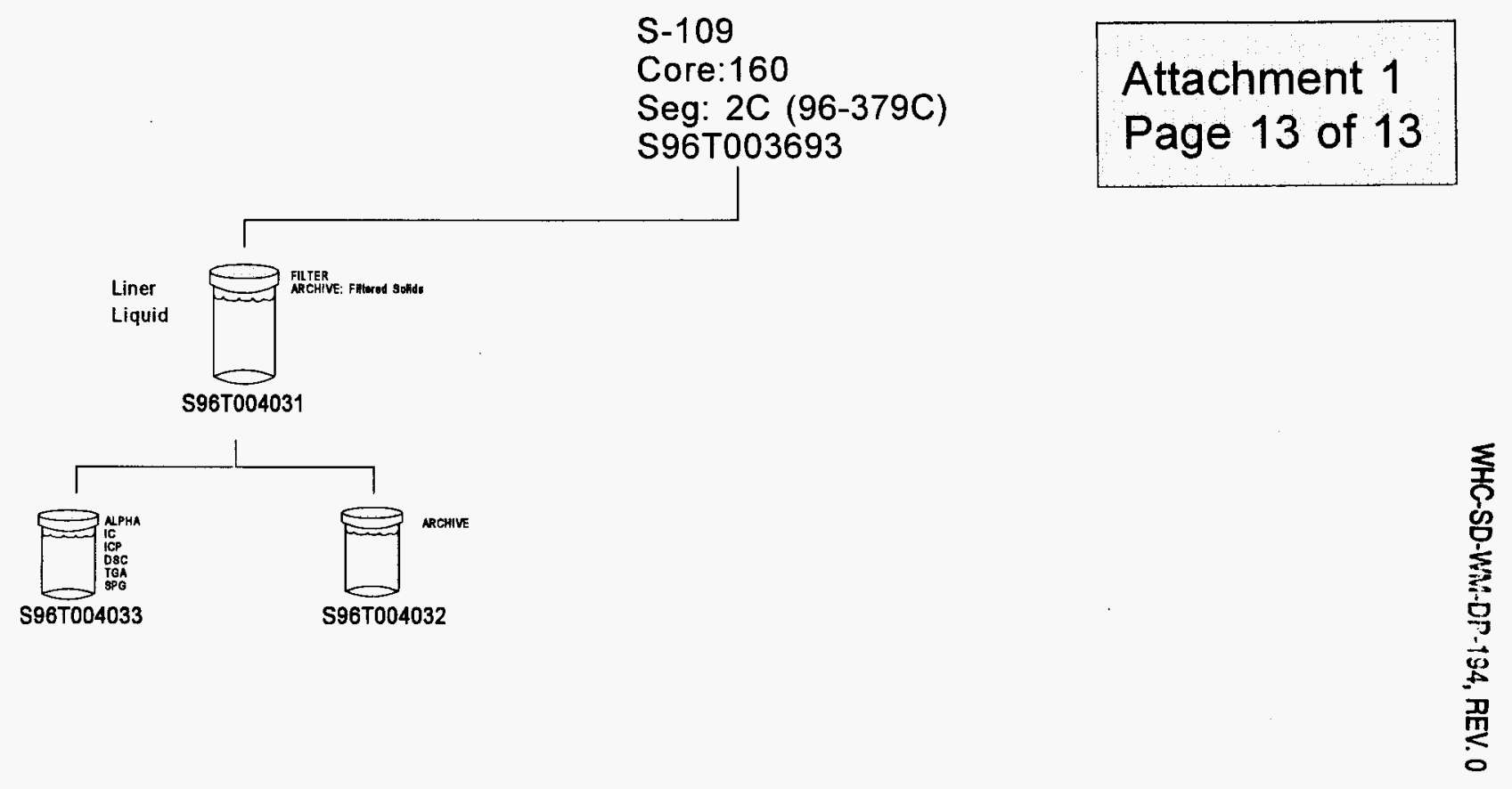


WHC-SD-WM-DP-194, REV. 0

SAMPLE DATA SUMMARY

28 
WHC-SD-WM-DP-194, REV. 0

THIS PAGE WAS INTENTIONALLY LEFT BLANK

29 
CORE MUMBER: 160

SEGMENT \#; Field Blank

SEGMENT PORTION: Field Blank

\begin{tabular}{|c|c|c|c|c|c|c|c|c|c|c|c|c|c|c|}
\hline & & & & Action & imits & & & & & & & & & \\
\hline Samplez & RlAH & Analyte & Unit & Lower & toper & Standard \% & Blank & Result & Dup & Averaqe & RPD & Spk Rec $z$ : & Limit & unt Err\% \\
\hline 5961004023 & & DSC Exotherm using Mettler & Joules/g & $-1.0 \mathrm{e}+00$ & 86 & 94.55 & $\mathrm{n} / \mathrm{a}$ & $0.00 \mathrm{e}+00$ & $0.00 \mathrm{e}+00$ & $0.00 \mathrm{e}+00$ & 0.00 & $n / a$ & $n / a$ & $n / a$ \\
\hline 5961004023 & & DSC Exotherm Dry Calculated & Joules/g Dry & $-1.0 e+00$ & $\%$ & $n / a$ & n/a & $0.00 \mathrm{e}+00$ & $0.00 \mathrm{e}+00$ & $0.00 \mathrm{e}+00$ & 0.00 & $n / a$ & $n / a$ & $n / a$ \\
\hline 5961004023 & & $\%$ Water by TGA using Mettler & $\%$ & None & None & 99.88 & $n / a$ & 99.98 & 99.86 & 99.92 & 0.12 & $n / a$ & $\mathrm{n} / \mathrm{a}$ & $n / a$ \\
\hline 5967004023 & & Alpha in Liquid Samples & UCi/mL & $-1.0 \mathrm{e}+00$ & 8 & 93.75 & $3.88 \mathrm{e}-06$ & $<6.61 \mathrm{e}-06$ & $3.58 \mathrm{e}-06$ & n/a & $n / a$ & a & $e-06$ & +02 \\
\hline
\end{tabular}

$\Rightarrow$ Limit violated

$\Rightarrow$ Selected Limit

๕̊ 
CORE NUMBER: 158

SEGMENT \#: 1

SEGMENT PORTION: U Upper Half of Segment

\begin{tabular}{|c|c|c|c|c|c|c|c|c|c|c|c|c|c|c|}
\hline & & & & Action & Limits & & & & & & & & & \\
\hline Samole\# & $R \mid$ A\# & Analyte & Unit & Lower & Upper & standard \% & Blank & Result & Dupl icate & Average & RPD \% & Spk Rec \% & Det Limit & Err\% \\
\hline S96I003916 & & Bulk Density of Sample & $\mathrm{g} / \mathrm{mL}$ & None & Hone & $n / a$ & $\mathrm{n} / \mathrm{a}$ & 1.730 & $\mathrm{n} / \mathrm{a}$ & $\mathrm{n} / \mathrm{a}$ & $\mathrm{n} / \mathrm{a}$ & $n / a$ & $5.00 e-01$ & $n / a$ \\
\hline $596 \mathrm{~T} 003922$ & & DSC Exotherm Dry Calculated & Joules/g Dry & $-1.0 \mathrm{e}+00$ & (6id & $\mathrm{n} / \mathrm{a}$ & $\mathrm{n} / \mathrm{a}$ & $0.00 \mathrm{e}+00$ & $0.00 \mathrm{e}+00$ & $0.00 \mathrm{e}+00$ & 0.00 & $\mathrm{n} / \mathrm{a}$ & $n / a$ & $n / a$ \\
\hline \$96T003922 & & DSC Exotherm on Perkin Elmer & Joules/g & $-1.0 \mathrm{e}+00$ & $\mathrm{HBO}$ & 94.03 & $\mathrm{n} / \mathrm{a}$ & $0.00 \mathrm{e}+00$ & $0.00 \mathrm{e}+00$ & $0.00 \mathrm{e}+00$ & 0.00 & n/a & $n / a$ & $n / a$ \\
\hline 5961003922 & & $\%$ Water by TGA on Perkin Elmer & $\%$ & None & Hone & 100.0 & $\mathrm{n} / \mathrm{a}$ & 18.90 & 27.27 & 23.09 & 36.3 & $n / a$ & $n / a$ & $n / a$ \\
\hline $596 \mathrm{~T} 003935$ & $F$ & Alpha of Digested Solid & uCi/g & $-1.0 \mathrm{e}+00$ & 8 & 117.2 & $<2.01 \mathrm{e}-03$ & $1.03 \mathrm{e}-02$ & $6.48 \mathrm{e}-03$ & $8.39 \mathrm{e}-03$ & 45.5 & $n / a$ & $5.00 \mathrm{e}-03$ & $=01$ \\
\hline
\end{tabular}

$L$ Lower Half of Segment: L Lower Half of Segment

\begin{tabular}{|c|c|c|c|c|c|c|c|c|c|c|c|c|c|c|}
\hline & & & & Action & imits & & & & & & & & & \\
\hline Sample\# & A\# & Analyte & Unit & Lower & Upper & Standard \% & Blank & Result & Dupl icate & Average & RPD $\%$ & pk Rec \% & Det Limit & Count Err\% \\
\hline $596 T 003917$ & & Bulk Density of Sample & $\mathrm{g} / \mathrm{mL}$ & Mone & None & $n / a$ & $n / a$ & 1.340 & n/a & $\mathrm{n} / \mathrm{a}$ & $n / a$ & n/a & $5.00 e-01$ & $n / a$ \\
\hline 5961003923 & & DSC Exotherm Dry calculated & Joules/g Dry & $-1.0 \mathrm{e}+00$ & $\%$ & n/a & $\mathrm{n} / \mathrm{a}$ & $0.00 \mathrm{e}+00$ & $0.00 \mathrm{e}+00$ & $0.00 \mathrm{e}+00$ & 0.00 & $n / a$ & $n / a$ & $n / a$ \\
\hline $596 r 003923$ & & DSC Exotherm on Perkin Elmer & Joules/g & $-1.0 \mathrm{e}+00$ & H.6\% & 94.03 & $\mathrm{n} / \mathrm{a}$ & $0.00 \mathrm{e}+00$ & $0.00 \mathrm{e}+00$ & $0.00 \mathrm{e}+00$ & 0.00 & n/a & $\mathrm{n} / \mathrm{a}$ & $n / a$ \\
\hline 5961003923 & & $\%$ Water by TGA on Perkin Elmer & $\%$ & None & Mone & 100.0 & $n / a$ & 7.190 & 7.210 & 7.200 & 0.28 & $\mathrm{n} / \mathrm{a}$ & $n / a$ & $\mathrm{n} / \mathrm{a}$ \\
\hline 5967003936 & $F$ & Alpha of Digested Solid & $\mathrm{uCi} / \mathrm{g}$ & $-1.0 \mathrm{e}+00$ & 35 & 117.2 & $<2.01 \mathrm{e}-03$ & $2.24 e-02$ & $2.03 e-02$ & $2.14 \mathrm{e}-02$ & 9.84 & n/al & $5.00 e-03$ & $2.67 \mathrm{E}+01$ \\
\hline
\end{tabular}

$\Rightarrow$ Limit violated

$\Rightarrow$ Selected Limit

$\omega$ 


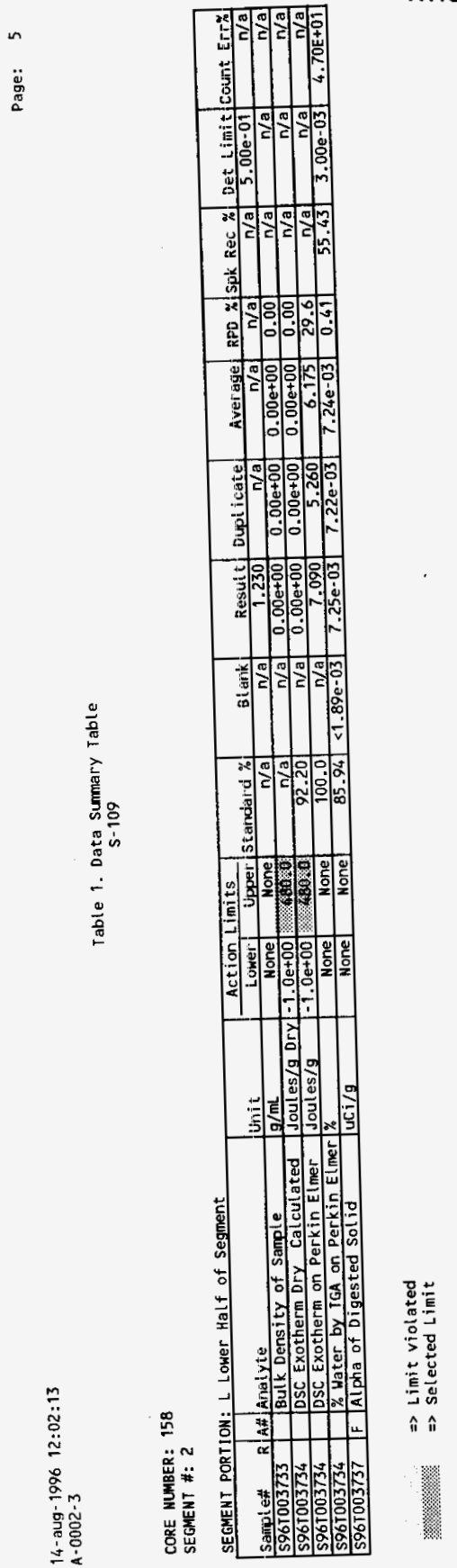


Table 1. Data Sumary Table

S-109

CORE NUMBER: 158

SEGMENT \#: 2 A

SEGMENT PORTION: L LOWer Half of Segment

\begin{tabular}{|c|c|c|c|c|c|c|c|c|c|c|c|c|c|c|}
\hline & & & & Action & imits & & & & & & & & & \\
\hline Samplan & $A=$ & Analyte & Init & Loher & lyper & Standard \% & R!ank! & Result & Dunl icate & Average & RPD \% & Spk Rec \% & Det Limit & Count Err\% \\
\hline$\$ 961004017$ & & DSC Exotherm using Mettler & Joules/g & $-1.0 \mathrm{e}+00$ & 8 & 99.12 & n/a & 31.70 & 47.80 & 39.75 & 40.5 & $n / a$ & $\mathrm{n} / \mathrm{a}$ & $n / a$ \\
\hline$\$ 961004017$ & & DSC Exotherm Dry Calculated & Joules/g Dry & $-1.0 \mathrm{e}+00$ & Sto & $n / a$ & $n / a$ & 34.30 & 51.70 & 43.00 & 40.5 & n/a & $\mathrm{n} / \mathrm{a}$ & $n / a$ \\
\hline S9690 & & $\%$ Water by TGA using Mettler & $\%$ & Hone & None & 99.90 & $\mathrm{n} / \mathrm{a}$ & 7.790 & 7.410 & 7.600 & 5.00 & $\mathrm{n} / \mathrm{a}$ & $\mathrm{n} / \mathrm{a}$ & $n / a$ \\
\hline 967004018 & F & Alpha of Digested Solid & $\mathrm{uCi} / \mathrm{g}$ & $-1.0 \mathrm{e}+00$ & 85 & 117.2 & $<2.01 e-03$ & $8.74 e^{-03}$ & $4.41 e-03$ & $6.57 e-03$ & 65.9 & $\mathrm{n} / \mathrm{a}$ & $5.00 \mathrm{e}-03$ & $.04 \mathrm{E}+01$ \\
\hline
\end{tabular}

$\quad \Rightarrow$ Limit violated

$\Rightarrow$ Selected Limit

$\stackrel{\omega}{\omega}$ 
CORE NUMBER: 158

SEGMENT \#: $2 B$

SEGMENT PORTION: U Upper Half of Segment

\begin{tabular}{|c|c|c|c|c|c|c|c|c|c|c|c|c|c|c|}
\hline & & & & Action & imits & & & & & & & & & \\
\hline Sanp! en & An & Analyte & Unit & Louner & Upoer & Standard \% & Btank & Result & Dupl icate & Average & RPD \% & Spk Rec *] & Det Limit & Count Err\% \\
\hline 5961003757 & & Bulk Density of Sample & $g /$ तit & None & None & $n / a$ & $n / a$ & 1.370 & $n / a$ & $n / a$ & $n / a$ & $n / a$ & $5.00 \mathrm{e}-01$ & $n / a$ \\
\hline $596 \mathrm{~T} 003759$ & & DSC Exotherm Dry Calculated & Joules/g Dry & $-1.0 e+00$ & 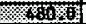 & $n / a$ & $n / a$ & $0.00 \mathrm{e}+00$ & $0.00 \mathrm{e}+00$ & $0.00 \mathrm{e}+00$ & 0.00 & $n / a$ & $n / a$ & $n / a$ \\
\hline 3759 & & DSC Exotherm on Perkin Elmer & Joutes/g & $-1.0 \mathrm{e}+00$ & 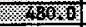 & 92.20 & $n / a$ & $0.00 \mathrm{e}+00$ & $0.00 \mathrm{e}+00$ & $0.00 e+00$ & 0.00 & $\mathrm{n} / \mathrm{a}$ & $n / a$ & $\mathrm{n} / \mathrm{a}$ \\
\hline 5967003759 & & \% Water by TGA on Perkin Elmer & $\%$ & None & None & 100.0 & $\mathrm{n} / \mathrm{a}$ & 11.03 & 10.44 & 10.73 & 5.50 & $n / a$ & $n / a$ & $n / a$ \\
\hline 5967003763 & $\mathrm{~F}$ & Alpha of Digested Solid & $\mathrm{uCi} / \mathrm{g}$ & $-1.0 \mathrm{e}+00$ & 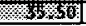 & 117.2 & $01 e-03$ & $2.63 \mathrm{e}-03$ & $5.57 \mathrm{e}-03$ & $4.10 \mathrm{e}+03$ & 71.7 & 83.29 & $5.00 \mathrm{e}-03$ & +02 \\
\hline
\end{tabular}

L Lower Half of Segment: L Lower Half of Segment

\begin{tabular}{|c|c|c|c|c|c|c|c|c|c|c|c|c|c|c|}
\hline \multirow[b]{2}{*}{ Sample\# R } & \multirow[b]{2}{*}{$\mathbf{R}$ A\# } & \multirow[b]{2}{*}{ Analyte } & \multirow[b]{2}{*}{ Unit } & \multicolumn{2}{|c|}{ Action Limits } & \multirow[b]{2}{*}{ Standard \% } & \multirow[b]{2}{*}{ Blank } & \multirow[b]{2}{*}{ Resul $t$} & \multirow[b]{2}{*}{ Dupl icate } & \multirow[b]{2}{*}{ Average } & \multirow[b]{2}{*}{ RPD \% } & \multirow[b]{2}{*}{ Spk Rec \% } & \multirow[b]{2}{*}{ Det Limit } & \multirow[b]{2}{*}{ Count Err\% } \\
\hline & & & & Lower & Upper & & & & & & & & & \\
\hline 5967003758 & & Bulk Density of Sample & $g / 11 \mathrm{~mL}$ & None & Hone & $n / a$ & $n / a$ & insuff.s & $n / a$ & $n / a$ & $n / a$ & n/a & $5.00 e-01$ & $n / a$ \\
\hline 5967003760 & & DSC Exotherm us ing Mettler & Joules/g & $-1.0 \mathrm{e}+00$ & 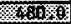 & 91.74 & $n / a$ & $8.00 \mathrm{e}-01$ & 1.100 & $9.50 \mathrm{e}-01$ & 31.6 & $\mathrm{n} / \mathrm{a}$ & $n / a$ & $n / a$ \\
\hline 5967003760 & & DSC Exotherm Dry Calculated & Joules/g Dry & $-1.0 \mathrm{e}+00$ & \% & $\mathrm{n} / \mathbf{a}$ & $n / a$ & $9.00 \mathrm{e}-01$ & 1.200 & 1.050 & 28.6 & $n / a$ & $n / a$ & $n / a$ \\
\hline S96T003760 & & $\%$ Water by TGA using Mettler & $\%$ & None & Mone & 98.72 & $n / a$ & 10.50 & 12.08 & 11.29 & 14.0 & $n / a$ & $n / a$ & $n / a$ \\
\hline \$96T003764 & $F$ & Alpha of Digested Solid & $\mathrm{uCi} / \mathrm{g}$ & $-1.0 \mathrm{e}+00$ & 56 & 86.72 & $<1.61 e^{-03}$ & $<3.09 \mathrm{e}-03$ & $2.95 e-03$ & $n / a$ & $\mathrm{n} / \mathrm{a}$ & 61.56 & $4.00 e-03$ & $1.89 E+02$ \\
\hline
\end{tabular}

$\Rightarrow$ Limit violated

$\Rightarrow$ Selected Limit

W 
CORE NUMBER: 158

SEGMENT \#: 3

SEGMENT PORTION: U Upper Half of Segment

\begin{tabular}{|c|c|c|c|c|c|c|c|c|c|c|c|c|c|c|}
\hline & & & & Action & Limits & & & & & & & & & \\
\hline Sample\# & $\mathrm{R}$ A\# & Analvte & Unit & Lower & Unmer & st andard $\%$ & B! ank & Result t & Dupl icate & Average & RPD $\times$ & Sọk Rec \% & Det Limit & Count Err\% \\
\hline 5961003918 & & Bulk Density of Sample & g/GL & None & None & $n / a$ & $\mathrm{n} / \mathrm{a}$ & 1.280 & $\mathrm{n} / \mathrm{a}$ & $\mathrm{n} / \mathrm{a}$ & $n / a$ & $\mathrm{n} / \mathrm{a}$ & $5.00 \mathrm{e}-01$ & $n / a$ \\
\hline 5967003924 & & DSC Exotherm Dry calculated & Joules/g Dry & $-1.0 \mathrm{e}+00$ & S60 & $n / a$ & n/a & $0.00 \mathrm{e}+00$ & $0.00 \mathrm{e}+00$ & $0.00 \mathrm{e}+00$ & 0,00 & $\mathrm{n} / \mathrm{a}$ & $n / a$ & $n / a$ \\
\hline 5967003924 & & DSC Exotherm on Perkin Elmer & Joules/g & $-1.0 \mathrm{e}+00$ & $860 \%$ & 94.90 & $n / a$ & $0.00 \mathrm{e}+00$ & $0.00 \mathrm{e}+00$ & $0.00 \mathrm{e}+00$ & 0.00 & $n / a$ & $n / a$ & $\mathrm{n} / \mathrm{a}$ \\
\hline 5967003924 & & \% water by TGA on Perkin Elmer & $\%$ & None & None & 100.0 & $n / a$ & 7.150 & 6.690 & 6.920 & 6.65 & $\mathrm{n} / \mathrm{a}$ & $\mathrm{n} / \mathrm{a}$ & $n / a$ \\
\hline $596 T 003937$ & $\mathbf{F}$ & Alpha of Digested Solid & $u C i / g$ & $-1.0 \mathrm{e}+00$ & 5 & 86.72 & $<1.61 \mathrm{e}-03$ & $5.56 \mathrm{e}-03$ & $<5.02 E-3$ & $\mathrm{n} / \mathrm{a}$ & $n / a$ & $n / a$ & $4.00 \mathrm{e}-03$ & $6.59 \mathrm{E}+01$ \\
\hline
\end{tabular}

L Lower Half of Segment: L Lower Half of Segment

\begin{tabular}{|c|c|c|c|c|c|c|c|c|c|c|c|c|c|c|}
\hline & & & & Action & Limits & & & & & & & & & \\
\hline Sample\# & A\# & Analyte & Unit & Lower & Upper & Standard \% & Blank & Result & Dupl icate & Average & RPD \% & Spk Rec \% & Det Limit & at Err\% \\
\hline 5967003919 & & Bulk Density of Sample & $g / B \mathrm{BL}$ & None & None & $n / a$ & $n / a$ & 1.590 & $\mathrm{n} / \mathrm{a}$ & $\mathrm{n} / \mathrm{a}$ & $\mathrm{n} / \mathrm{a}$ & $n / a$ & $5.00 e-01$ & $n / a$ \\
\hline S96T003925 & & DSC Exotherm Dry calculated & Joules/g Dry & $-1.0 \mathrm{e}+00$ & 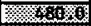 & $\mathrm{n} / \mathrm{a}$ & $n / a$ & $0.00 \mathrm{e}+00$ & $0.00 \mathrm{e}+00$ & $0 . \overline{00} e+00$ & 0.00 & $\mathrm{n} / \mathrm{a}$ & $\mathrm{n} / \mathrm{a}$ & $n / a$ \\
\hline S96T003925 & & DSC Exotherm on Perkin Eimer & Joules/g & $-1.0 \mathrm{e}+00$ & 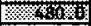 & 94.90 & $n / a$ & $0.00 \mathrm{e}+00$ & $0.00 \mathrm{e}+00$ & $0.00 \mathrm{e}+00$ & 0.00 & $n / a$ & $\mathrm{n} / \mathrm{a}$ & $n / a$ \\
\hline S96T003925 & & \% Hater by TGA on Perkin Elmer & $\%$ & None & None & 100.0 & $n / a$ & 12.79 & 10.74 & 11.77 & 17.4 & $n / a$ & $\mathrm{n} / \mathrm{a}$ & $\mathrm{n} / \mathrm{a}$ \\
\hline \$96T003938 & $\mathrm{F}$ & Alpha of Digested Solid & $u c i / g$ & $-1.0 \mathrm{e}+00$ & 5 & 86.72 & $<1.61 \mathrm{e}-03$ & $6.11 \mathrm{e}-03$ & $8.36 e-03$ & $7.24 \mathrm{e}-03$ & 31.1 & $n / a$ & $4.00 \mathrm{e}-03$ & $.80 \mathrm{E}+01$ \\
\hline
\end{tabular}

$\Rightarrow$ Limit violated

$\Rightarrow$ Selected Limit

$\omega$ 
Table 1. Data Sumary Table

S-109

CORE NUMBER: 158

SEGMENT \#: $3 A$

\begin{tabular}{|c|c|c|c|c|c|c|c|c|c|c|c|c|c|c|}
\hline & & & & Action & Limits & & & & & & & & & \\
\hline Samplent & A & Anolyte & Unit & Lower & Upper & Standard $\%$ & Btonk & Result: & Dupl icatel & Average & RPD \% & Spk nec $\%$ & Det Limit & Count Erry \\
\hline 5967003920 & & Bulk Density of Sample & $\mathrm{g} / \mathrm{mL}$ & None & None & n/a & $n / a$ & 1.340 & n/a & n/a & n/a & $n / a$ & $5.00 e-01$ & $\mathrm{n} / \mathrm{a}$ \\
\hline 5967003926 & & DSC Exotherm using Mettler & Joules $/ g$ & $-1.0 \mathrm{e}+00$ & (3) & 92.09 & $\mathrm{n} / \mathrm{a}$ & 1.200 & $0.00 \mathrm{e}+00$ & $6.00 \mathrm{e}-01$ & 200 & n/a & $n / a$ & $n / a$ \\
\hline 5967003926 & & DSC Exotherm Dry calculated & Joules/g Dry & $-1.0 e+00$ & 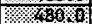 & $n / a$ & $n / a$ & 1.300 & $0.00 \mathrm{e}+00$ & $6.50 e-01$ & 200 & $n / a$ & $\mathrm{n} / \mathrm{a}$ & $n / a$ \\
\hline 5967003926 & & \% Water by IGA using Mettler & $\%$ & None & None & 98.53 & $n / a$ & 9.200 & 8.700 & 8.950 & 5.59 & $n / a$ & $n / a$ & $\mathrm{n} / \mathrm{a}$ \\
\hline S96T003939 & $F$ & Alpha of Digested solid & uCi $/ g$ & $-1.0 \mathrm{e}+00$ & 3 & 86.72 & $<1.61 e^{-03}$ & $3.03 e-03$ & $8.55 e-03$ & $5.79 e-03$ & 95.3 & $n / a$ & $4.00 \mathrm{e}-03$ & $1.17 \mathrm{E}+02$ \\
\hline
\end{tabular}

$\Rightarrow$ Limit violated
$\Rightarrow$ Selected Limit

$\stackrel{\omega}{\circ}$ 
CORE MUMBER: 158

SEGMEHT \#: 4

SEGMENT PORTION: L Lower Half of Segment

\begin{tabular}{|c|c|c|c|c|c|c|c|c|c|c|c|c|c|c|}
\hline \multirow{2}{*}{\multicolumn{2}{|c|}{ Sampl ett R }} & \multirow[b]{2}{*}{ Analyte } & \multirow[b]{2}{*}{ Unit } & \multicolumn{2}{|c|}{ Action Limits } & \multirow[b]{2}{*}{ Standard \& } & \multirow[b]{2}{*}{ Q! ank } & \multirow[b]{2}{*}{ Result } & \multirow[b]{2}{*}{ Dunt icate } & \multirow[b]{2}{*}{ Average } & \multirow[b]{2}{*}{ ppD $x$} & \multirow[b]{2}{*}{ Spk Rec \% } & \multirow[b]{2}{*}{ Det limit } & \multirow[b]{2}{*}{ Count Err\% } \\
\hline & R Af & & & Loher & Inoner & & & & & & & & & \\
\hline 5967003921 & & Bulk Density of Sample & $\mathrm{g} / \mathrm{mL}$ & None & None & $n / a$ & $n / a$ & 1.270 & $n / a$ & $\mathrm{n} / \mathrm{a}$ & $n / a$ & $n / a$ & $5.00 e-01$ & $\mathrm{n} / \mathrm{a}$ \\
\hline 596T003927 & & DSC Exotherm using Mettler & Joules/g & $-1.0 \mathrm{e}+00$ & Kon & 92.09 & $\mathrm{n} / \mathrm{a}$ & $0.00 \mathrm{e}+00$ & 30.50 & 15.25 & 200 & $\mathrm{n} / \mathrm{a}$ & $n / a$ & $n / a$ \\
\hline \$96T003927 & & DSC Exotherm Dry Calculated & Joules/gDry & $-1.0 \mathrm{e}+00$ & 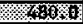 & $n / a$ & $\mathrm{n} / \mathrm{a}$ & $0.00 \mathrm{e}+00$ & 32.50 & 16.25 & 200 & $n / 2$ & $n / a$ & $\mathrm{n} / \mathrm{a}$ \\
\hline \$967003927 & & \% Water by IGA using Mettler & $\%$ & None & None & 98.53 & $\mathrm{n} / \mathrm{a}$ & 6.000 & 6.100 & 6.050 & 1.65 & $n / a$ & $n / a$ & $\mathrm{n} / \mathrm{a}$ \\
\hline S96T003940 & $\mathbf{F}$ & Alpha of Digested Solid & $u C i / g$ & $-1.0 \mathrm{e}+00$ & 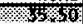 & 91.41 & $1.00 \mathrm{e}-03$ & $9.99 \mathrm{e}-03$ & $8.40 \mathrm{e}-03$ & $9.20 \mathrm{e}-03$ & 17.3 & 51.53 & $1.00 e-03$ & $2.47 E+01$ \\
\hline
\end{tabular}

$\Rightarrow$ Limit violated

$\Rightarrow$ Selected Limi 
Table 1. Data Summary Table

$$
\text { s-109 }
$$

CORE NUNBER: 160

SEGMENI \#; 1

SEGMENT PORTION: L Lower Half of Segment

\begin{tabular}{|c|c|c|c|c|c|c|c|c|c|c|c|c|c|c|}
\hline \multirow[b]{2}{*}{ Sanplen $R$} & \multirow[b]{2}{*}{ AH } & \multirow{2}{*}{ Añalyte } & \multirow[b]{2}{*}{ Uini t } & \multicolumn{2}{|c|}{ Action Limits } & \multirow[b]{2}{*}{ Standard $\%$} & \multirow[b]{2}{*}{$\bar{B} i$ ank } & \multirow[b]{2}{*}{ Kesuit } & \multirow[b]{2}{*}{ Dupi icate } & \multirow[b]{2}{*}{ Áverage } & \multirow[b]{2}{*}{$\bar{K} \bar{P} \bar{O} \hat{z}$} & \multirow[b]{2}{*}{ Spi R̄ec zi } & \multirow[b]{2}{*}{ Det Limit } & \multirow[b]{2}{*}{ Count Errô̆ } \\
\hline & & & & Lower & ipper & & & & & & & & & \\
\hline 5961003769 & & Bulk Density of Sample & $\mathrm{g} / \mathrm{mL}$ & None & None & $n / a$ & $n / a$ & 1.270 & $\mathrm{n} / \mathrm{a}$ & $n / a$ & $n / a$ & n/a & $5.00 \mathrm{e}-01$ & $\mathrm{n} / \mathrm{a}$ \\
\hline 5967003800 & & DSC Exotherm using Mettler & Joules/g & $-1.0 \mathrm{e}+00$ & 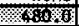 & 91.74 & $n / a$ & $0.00 \mathrm{e}+00$ & $0.00 \mathrm{e}+00$ & $0.00 \mathrm{e}+00$ & 0.00 & $n / a$ & $n / a$ & n/a \\
\hline S96T003800 & & DSC Exotherm Dry Calculated & Joules/g Dry & $-1.0 \mathrm{e}+00$ & (5) & $\mathrm{n} / \mathrm{a}$ & $\mathrm{n} / \mathrm{a}$ & $0.00 \mathrm{e}+00$ & $0.00 \mathrm{e}+00$ & $0.00 \mathrm{e}+00$ & 0.00 & $n / a$ & $\mathrm{n} / \mathrm{a}$ & $\mathrm{n} / \mathrm{a}$ \\
\hline 5961003800 & & $\%$ Water by TGA using Mettler & $\%$ & None & None & 98.72 & $n / a$ & 4.310 & $9.40 \mathrm{e}-01$ & 2.625 & 128 & $n / a$ & $n / a$ & $n / a$ \\
\hline 5961003815 & $F$ & Alpha of Digested Solid & $u C i / g$ & $-1.0 \mathrm{e}+00$ & 3. & 96.09 & $<1.22 \mathrm{e}-03$ & $3.29 \mathrm{e}-03$ & $4.18 \mathrm{e}-03$ & $3.73 e-03$ & 23.8 & 77.44 & $2.00 \mathrm{e}-03$ & $5.51 E+01$ \\
\hline
\end{tabular}

$\quad \Rightarrow$ Limit violated

$\mathscr{\leftrightarrow}$ 


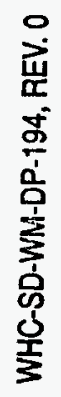

7 1 117 pazaajas < pazejola 3 ILI] $<=$

\begin{tabular}{|c|c|c|c|c|c|c|c|c|c|c|c|c|c|c|}
\hline $10+36 L \cdot \varepsilon$ & 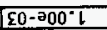 & 19.65 & $E_{L} \cdot 4$ & EO-วZद. 2 & $\overline{E O}-\partial \angle \Sigma^{*} \mathcal{L}$ & $50-395^{\circ} \cdot \overline{2}$ & $70-99 L^{\prime} \varepsilon>$ & 15.56 & DUON & DUON & $6 / ! 3 n$ & p! jos palsab!a to eld & & 718500196 \\
\hline E/4 & E/U & E/U & $8 . \varepsilon^{7}$ & $082 \cdot 1$ & $095 \% 1$ & $000 \% 1$ & E/4 & $S L \cdot 86$ & DUOH & गUON & $\%$ & 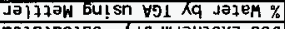 & & $\frac{108500196}{10960196}$ \\
\hline$E / \bar{U}$ & $e / 4$ & $\mathrm{e} / \mathrm{u}$ & 00.0 & $00+200^{\circ} 0$ & $100+200.0$ & $00+200^{\circ} 0$ & $\mathrm{E} / \mathrm{u}$ & $e / 4$ & 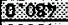 & $00+20^{-1}$ & Auq $6 /$ sajnor & 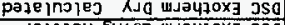 & & 0196 \\
\hline $\mathrm{e} / \mathrm{u}$ & $0 / 4$ & e/u & $00 \div 0$ & $00+200 \cdot 0$ & $00+200=0$ & $00+200^{\circ} 0$ & $\mathrm{E} / \mathrm{u}$ & 60.26 & Oxul & $00+20 \div=$ & E/szinor & 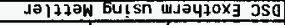 & & 60020011 \\
\hline e/u & $10-200^{\circ} \mathrm{s}$ & $8 / 4$ & $e / 4$ & $\mathrm{E} / \mathrm{u}$ & $\mathrm{E} / \mathrm{u}$ & $022 \% 1$ & E/U & $\mathrm{e} / \mathrm{u}$ & DUOH & OUON & $7 \omega / 5$ & ग0dues to Ki! süa ying & & $0 \angle L 200196$ \\
\hline \%ग19 7unos & $7 ! 4 ! 7700$ & \% ग्वे ऋds & \% Odd & DÉ̃JaAy & Prestiकo & finsay & 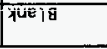 & \% PJEPUE TS & 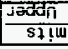 & $\begin{array}{l}\text { JaHO } \\
0 ! 17 P V\end{array}$ & F!un & 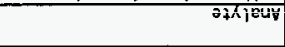 & & Hajolle \\
\hline
\end{tabular}

2 :\# 1 NJWOAS 09 : :y 


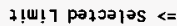
parejo!ุ มเพ!า <

\begin{tabular}{|c|c|c|c|c|c|c|c|c|c|c|c|c|c|}
\hline $10+312 \cdot 8$ & $20-200+7$ & Je/u & e/u & e/u & $\varepsilon-3\left|9^{\prime} \eta\right\rangle$ & $\sqrt{20-97_{7}^{*}}$ & $|20-955 \cdot 1\rangle$ & 60.96 & 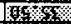 & $00+201=$ & $5 / ! 3 n$ & p!los palsas!a fo eqaly 1 & 0485001965 \\
\hline E/u & e/u & e/u & E:G2 & $598^{\circ} 2$ & $098^{-8}$ & $0<8^{\circ} 9$ & $\mathrm{e} / \mathrm{u}$ & 52.86 & BuOH & DUON & $\%$ & 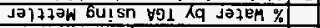 & $658 \varepsilon 00196 \mathrm{~S}$ \\
\hline$e / 4$ & $e / 4$ & e/u & 00.0 & $00+200.0$ & $00+200.0$ & $00+2000$ & E/u & $\mathrm{e} / \mathrm{u}$ & 10000 & $00+20 \div 1-$ & KJa $6 / \mathrm{sajnof}$ & 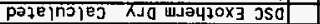 & $658900196 \mathrm{~S}$ \\
\hline $\mathrm{E} / \mathrm{u}$ & e/u & e/u & 00.0 & $00+200^{\circ} 0$ & $00+200^{\circ} 0$ & $00+200 \cdot 0$ & e/u & 60.26 & 9xon & $00+20.1-$ & 6/sajnop & 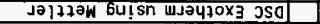 & 6585001965 \\
\hline$\%$ Jj了 tunioj & T!Ui! T Tau & 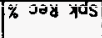 & $\overline{\text { Q }} \overline{\mathrm{d} d \bar{d}}$ & 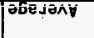 & 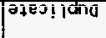 & tiñsay & Tive & $\overline{\%}$ PJEPLIEAS & 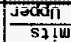 & $\frac{\text { Jomu i }}{\text { Uo!子Jy }}$ & +! पोi & गิरंpuy \#甘 & \#ânes \\
\hline
\end{tabular}

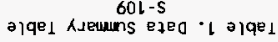


CORE NUM8ER: 160

SEGMENT \#: 28

SEGMENT PORIIION: U UPpper Half of Segment

\begin{tabular}{|c|c|c|c|c|c|c|c|c|c|c|c|c|c|c|}
\hline & & & & Action & Limits & & & & & & & & & \\
\hline Sample\# & $\left.\mathrm{R}\right|_{\text {A\# }}$ & Analyte & Unit & Lower & Upper & Standard \% & Blank & Result & Dupl icate & Average & RPO $x$ & $\mid$ Spk ReC $x_{0} \mid$ & Det Limit & Count Err\% \\
\hline 5967004028 & & Bulk Density of Sample & $\mathrm{g} / \mathrm{mL}$ & None & None & $\mathrm{n} / \mathrm{a}$ & $n / a$ & 1.190 & $\mathrm{n} / \mathrm{a}$ & $\mathrm{n} / \mathrm{a}$ & $\mathrm{n} / \mathrm{a}$ & $n / a$ & $5.00 e-01$ & $n / a$ \\
\hline S96T004035 & & DSC Exotherm using Mettler & Joules/g & $-1.0 e+00$ & 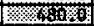 & 93.50 & $n / a$ & $0.00 \mathrm{e}+00$ & $0.00 e+00$ & $0.00 \mathrm{e}+00$ & 0.00 & $n / a$ & $n / a$ & $n / a$ \\
\hline S96T004035 & & DSC Exotherm Dry Calculated & Joules/g Dry & $-1.0 e+00$ & 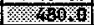 & $n / a$ & $n / a$ & $0.00 \mathrm{e}+00$ & $0.00 \mathrm{e}+00$ & $0.00 \mathrm{e}+00$ & 0.00 & $\mathrm{n} / \mathrm{a}$ & $n / a$ & $n / a$ \\
\hline $596 \mathrm{~T} 004035$ & & $\%$ Water by TGA using Mettler & $\%$ & None & None & 99.93 & $\mathrm{n} / \mathrm{a}$ & 5.950 & 6.100 & 6.025 & 2.49 & $n / a$ & $\mathrm{n} / \mathrm{a}$ & $n / a$ \\
\hline S96T004037 & $\mathbf{F}$ & Alpha of Digested Solid & $u C i / 9$ & $-1.0 \mathrm{e}+00$ & 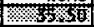 & 89.06 & $<7.92 \mathrm{e}-03$ & $5.43 \mathrm{e}-03$ & $6.50 \mathrm{e}-03$ & $5.96 \mathrm{e}-03$ & 17.9 & 60.17 & $3.00 \mathrm{e}-03$ & $6.28 E+01$ \\
\hline
\end{tabular}

\section{t}

\begin{tabular}{|c|c|c|c|c|c|c|c|c|c|c|c|c|c|c|}
\hline & & & & Action & imits & & & & & & & & & \\
\hline Sample\# & A\# & Analyte & Unit & Lower & Upper & Standard \% & Blank & Result & Dupl icate & Average & RPD $\%$ & Spk Rec \% & Det Limit & Count Err\% \\
\hline$\$ 967004029$ & & Bulk Density of Sample & g/mL & None & None & $n / a$ & $n / a$ & NA & n/a & $\mathrm{n} / \mathrm{a}$ & $n / a$ & n/a & $5.00 \mathrm{e}-01$ & $n / a$ \\
\hline 5967004036 & & DSC Exotherm using Mettler & Joules $/ g$ & $-1.0 \mathrm{e}+00$ & 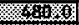 & 109.6 & $\mathrm{n} / \mathrm{a}$ & 9.500 & 8.900 & $\overline{9} .200$ & 6.52 & $n / a$ & $n / a$ & n/a \\
\hline 5967004036 & & DSC Exotherm Dry Calculated & Joules/g Dry & $-1.0 \mathrm{e}+00$ & 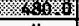 & $\mathrm{n} / \mathrm{a}$ & $n / a$ & 11.77 & 11.03 & 11.40 & 6.49 & n/a & $n / a$ & $n / a$ \\
\hline 5967004036 & & $\%$ water by TGA using Mettler & $\%$ & None & None & 100.2 & $\mathrm{n} / \mathrm{a}$ & 20.04 & 18.57 & 19.30 & 7.61 & $\mathrm{n} / \mathrm{a}$ & $\mathrm{n} / \mathrm{a}$ & $n / a$ \\
\hline S96T004038 & F & Alpha of bigested Solid & uci/g & $-1.0 \mathrm{e}+00$ & 35 & 89.06 & $<1.92 \mathrm{e}-03$ & $1.91 \mathrm{e}-02$ & $1.32 e-02$ & $1.61 \mathrm{e}-02$ & 36.5 & $n / a$ & $3.00 \mathrm{e}-03$ & $2.89 E+01$ \\
\hline
\end{tabular}

$\Rightarrow$ Limit violated

$\quad \Rightarrow$ Selected Limit

\section{出}


CORE MUMBER: 160 SEGMENT \#: $2 \mathrm{C}$

\begin{tabular}{|c|c|c|c|c|c|c|c|c|c|c|c|c|c|c|}
\hline & & & & Action & inits & & & & & & & & & \\
\hline Sample\# & R|A\# & Analyte & Unit & Lower & Upper & standard \% & Blank & Result & Dupl icate & Average & RPD $\%$ & Spk Rec \% & Det Limit & n \\
\hline \$96T004033 & & DSC Exotherm using Mettler & Joules $/ \mathrm{g}$ & $-1.0 \mathrm{e}+00$ & 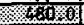 & 94.55 & $n / a$ & $0.00 \mathrm{e}+00$ & $0.00 \mathrm{e}+00$ & $0.00 \mathrm{e}+00$ & 0.00 & $\mathrm{n} / \mathrm{a}$ & $\mathrm{n} / \mathrm{a}$ & n \\
\hline 4033 & & DSC Exotherm Dry Calculated & Joules/g Dry & $-1.0 \mathrm{e}+00$ & 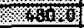 & $n / a$ & $\mathrm{n} / \mathrm{a}$ & $0.00 \mathrm{e}+00$ & $0.00 \mathrm{e}+00$ & $0.00 \mathrm{e}+00$ & 0.00 & $n / a$ & $n / a$ & $n$ \\
\hline 596T & & $\%$ Hater by IGA using Mettler & $\%$ & None & None & 99.88 & $\mathrm{n} / \mathrm{a}$ & 52.07 & 52.78 & 52.42 & 1.35 & $\mathrm{n} / \mathrm{a}$ & $n / a$ & $5+0$ \\
\hline & & 4 & UCi & $-1.0 e+00$ & 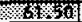 & 93.75 & $3.88 \mathrm{e}-06$ & $3.44 e-03$ & $4.14 e^{-04}$ & $1.93 e-0.3$ & 157 & 69.64 & $.32 e-04$ & $B E+C$ \\
\hline
\end{tabular}

\$96T004033 - \% Hater by IGA using Mettle

uCi/mL \begin{tabular}{l|r|r|}
\hline 69.64 & $2.32 e-04$ & $1.68 E+01$ \\
\hline
\end{tabular}

\section{$\Rightarrow$ Limit violated \\ $\Rightarrow$ Selected Limit}


WHC-SD-WM-DP-194, REV. 0

INORGANIC ANALYSES 
WHC-SD-WM-DP-194, REV. O

\section{THIS PAGE WAS INTENTIONALLY LEFT BLANK}


worklistrpt Version 2.1 05/15/95 WHC-SD-WM-DP-194, REV. 0

\section{LABCORE Data Entry Template for Worklist\#}

Analyst: $\quad$ KRM Instrument: $\mathrm{DSCO}_{3}$ Book \#12N14B

Method: LA-514-114 Rev/Mod $\quad \mathrm{C}-1$

Worklist Comment: S-109 DSC, RUN UNDER N2. RCJ

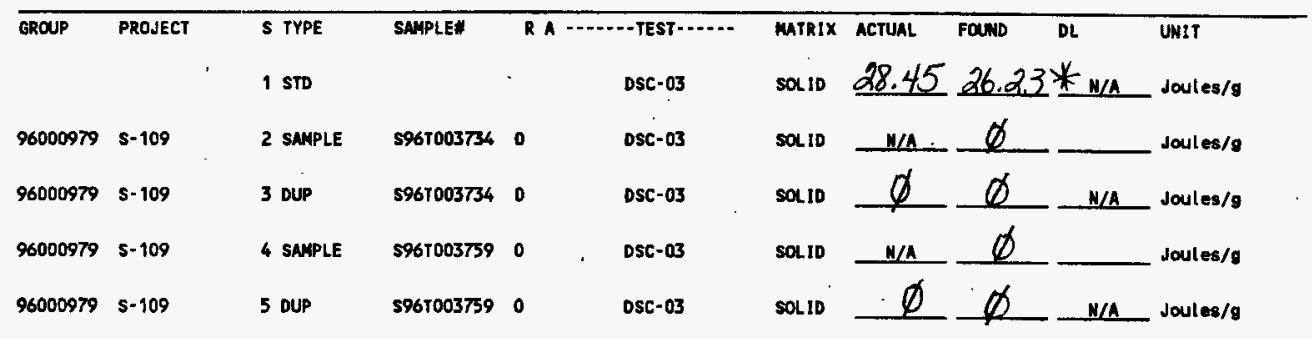

Ae attached lor signatures

Analyst Signature Date $\bigcup_{7-29-94}$

Verified/Validated bry

Blandina

Valenzuela $8-1-96$
11173

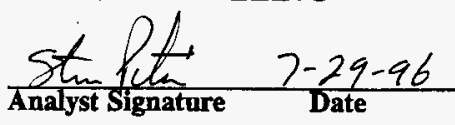

Data Entry Comments:

Units shown for $Q C$ (SPK \& STD) may not reflect the actual units. $D L=$ Detection Limit, $S=$ Worklist Slot Number, $\boldsymbol{R}=$ Replicate Number, $A=$ Aliquot Code. 


\section{LABCORE Data Entry Template for Worklist\#}

Analyst: KPM Instrument: DSC0 3 Book \# 12 NI4-B

Method: LA-514-113 Rev/Mod $C-1$

Worklist Comment: S-109 DSC, RUN UNDER N2. RCJ

\begin{tabular}{|c|c|c|c|c|c|c|c|c|c|c|}
\hline \multirow[t]{2}{*}{ GROUP } & PROJECT & S TYPE & SAMPLE\# & R A & ........TEST -...... & MATRIX & ACTUAL & FOUND & $\mathrm{DL}$ & UNIT \\
\hline & & 1 sto & & & DSC-01 & SOL.10 & & & $N / A$ & Joules/9 \\
\hline 96009979 & $s-109$ & 2 SAMPLE & 5961003734 & 0 & DSC-01 & SOLID & N/A & & & Joules/g \\
\hline 96000979 & s-109 & 3 DUP & s961003734 & 0 & DSC-01 & SOLID & & & $N / A$ & Joules/g \\
\hline 96000979 & s-109 & 4 SAMPLE & s96T003759 & 0 & DSC-01 & SOLID & $N / A$ & & & Joules/g \\
\hline 96000979 & $s-109$ & 5 DUP & s96T003759 & 0 & DSC-01 & SOLID & & & $H / A$ & Joules/g \\
\hline
\end{tabular}

\section{Analyst Signature Date}

\section{Final page for worklist \#}

\section{3}

\section{$7-28.96$}

\section{Analyst Signature Date}

$$
\text { DSC-03 Instrument was used. }
$$

Data Entry Comments:

Units shown for $Q C$ (SPK \& STD) may not reflect the actual wnits. $D L=$ Detection Limit, $S=$ Worklist Slot Number, $\boldsymbol{R}=$ Replicate Number, $A=$ Aliquot Cade. 
Cumve 1: DEC

F1le info: IMD07e801 Sun Jul 88 04: 50: 231996

Sample Weight: 19.750 ag

12N94-B INDIUN AT 10C/MIN

SICVATURE BELOW REPRESENTS CHEMICAL TECHNOLOGIST/CHEMIST THAT

COIALLETED/VERIFIED THE CALIBRATION/ANALYSIS ON PAGES $4 \geq$ TO $\Sigma \%$

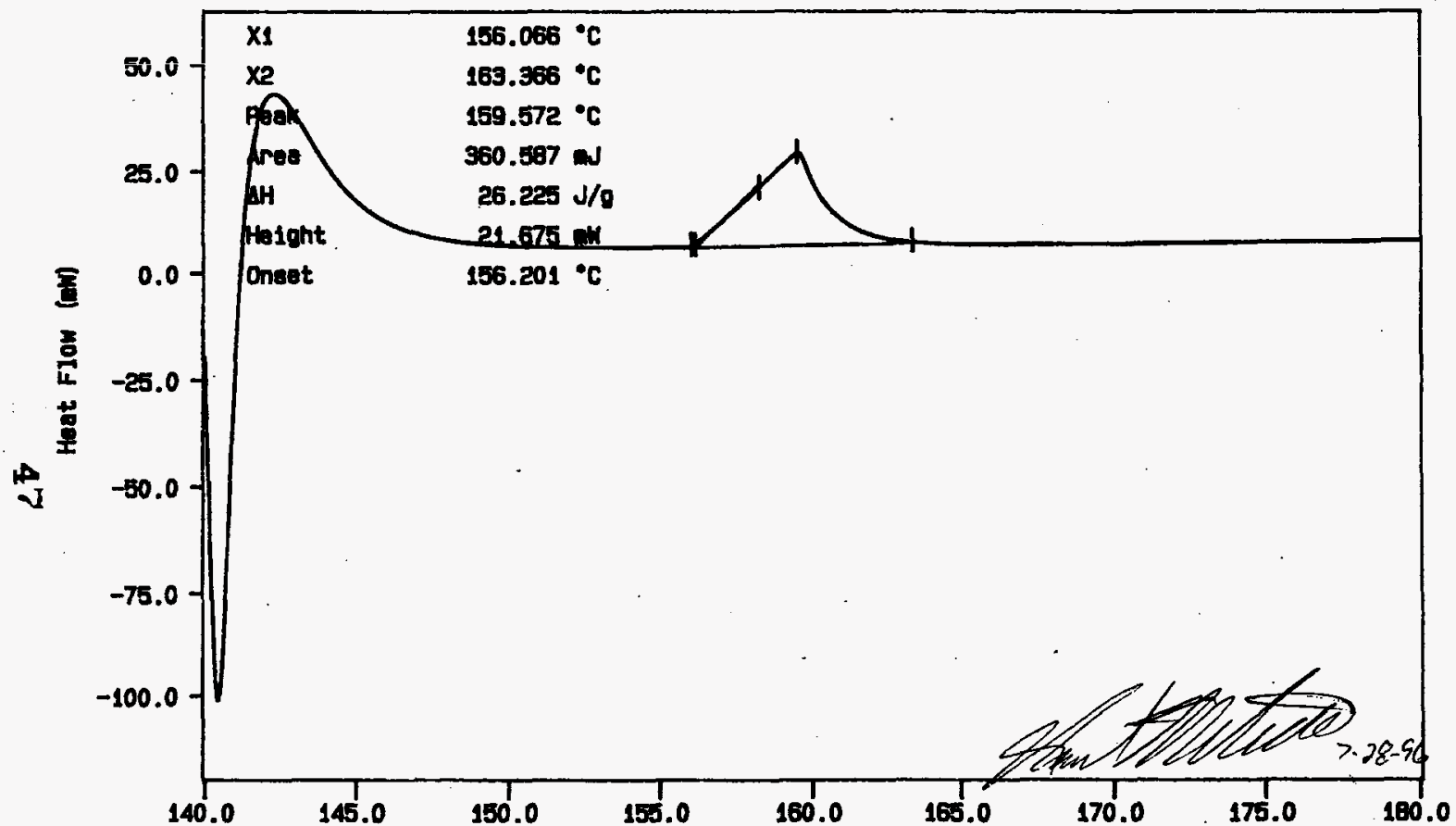

N2, EXOTHERM DOWN

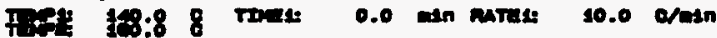

Temperature $\left(^{\circ} \mathrm{C}\right)$ KP MONTEITH

PERIN-Ely

7 Series Thermal Ansiysis Syatem 
WHC-SD-WM-DP-194, REV. 0

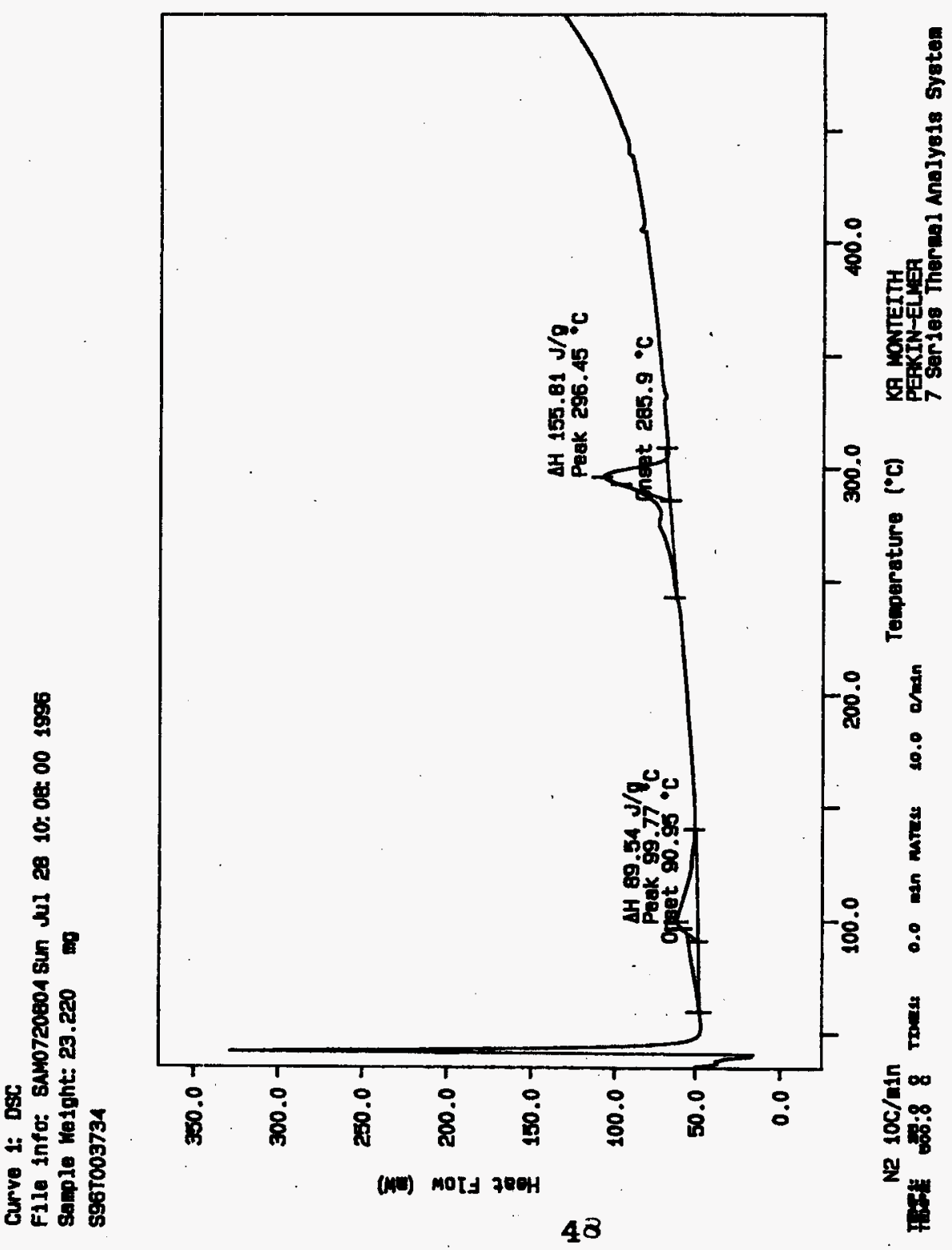


Curve 1: DSC

F1le Info: samp72805 sun wul 28 12 40:00 1996

Saple Weight: 25.720

S96T003734 DUP

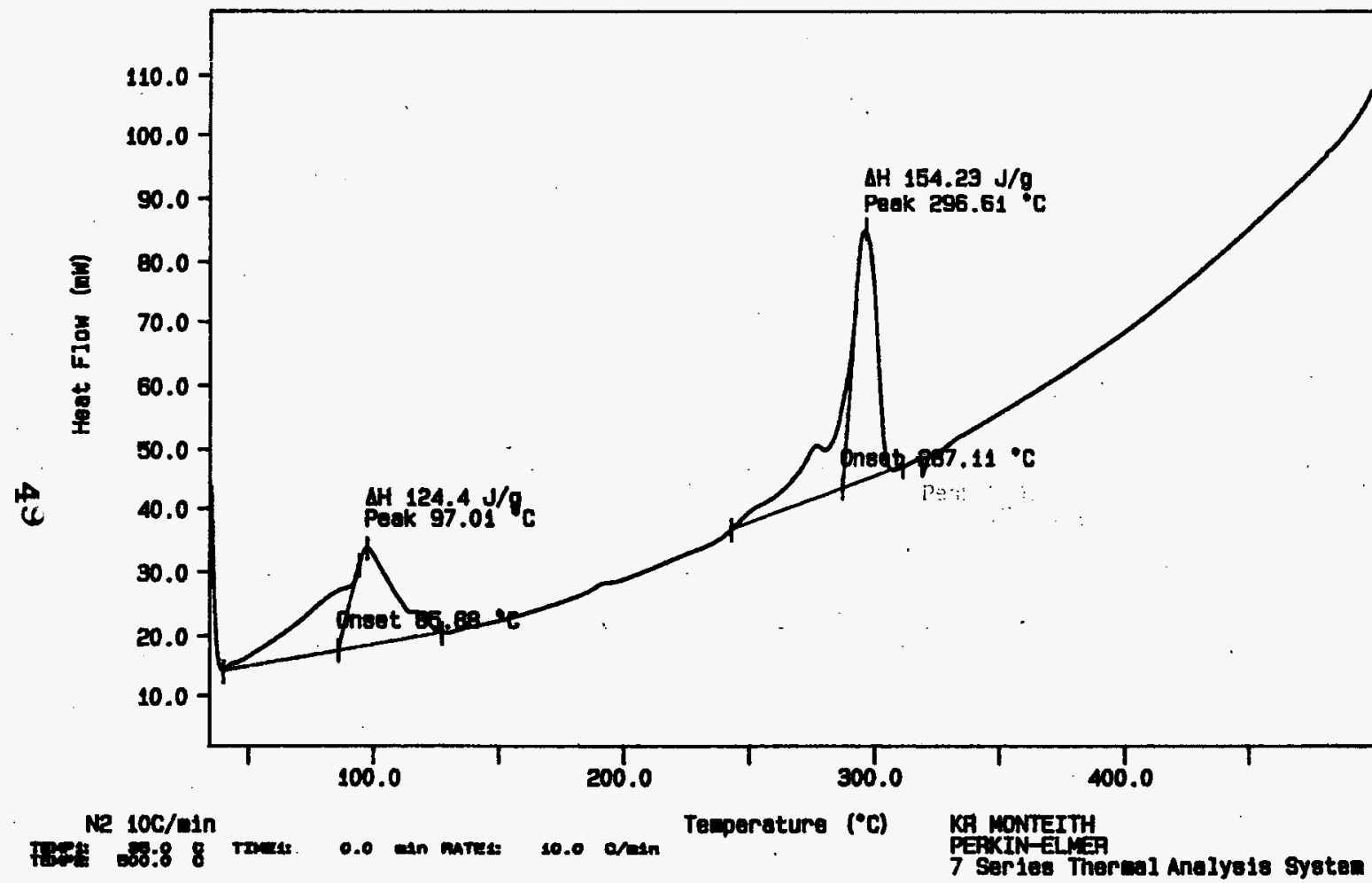


Curve i: DSC

F118 Info: samo7ea02 sun Jul 28 06: 23; 471996

Sanple Weight: 27.490

S96T003759

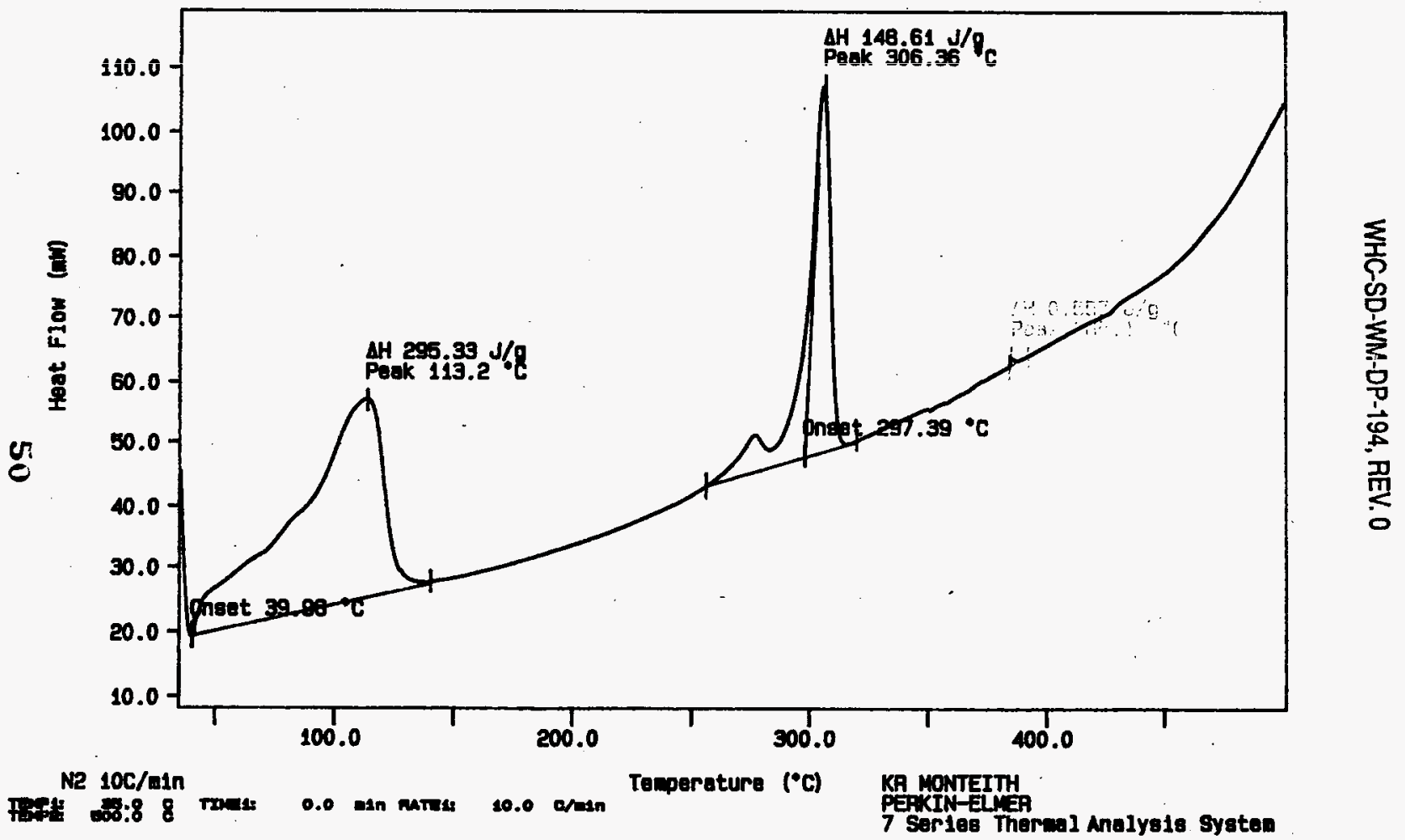


WHG-SD-WM-DP-194, REV. 0

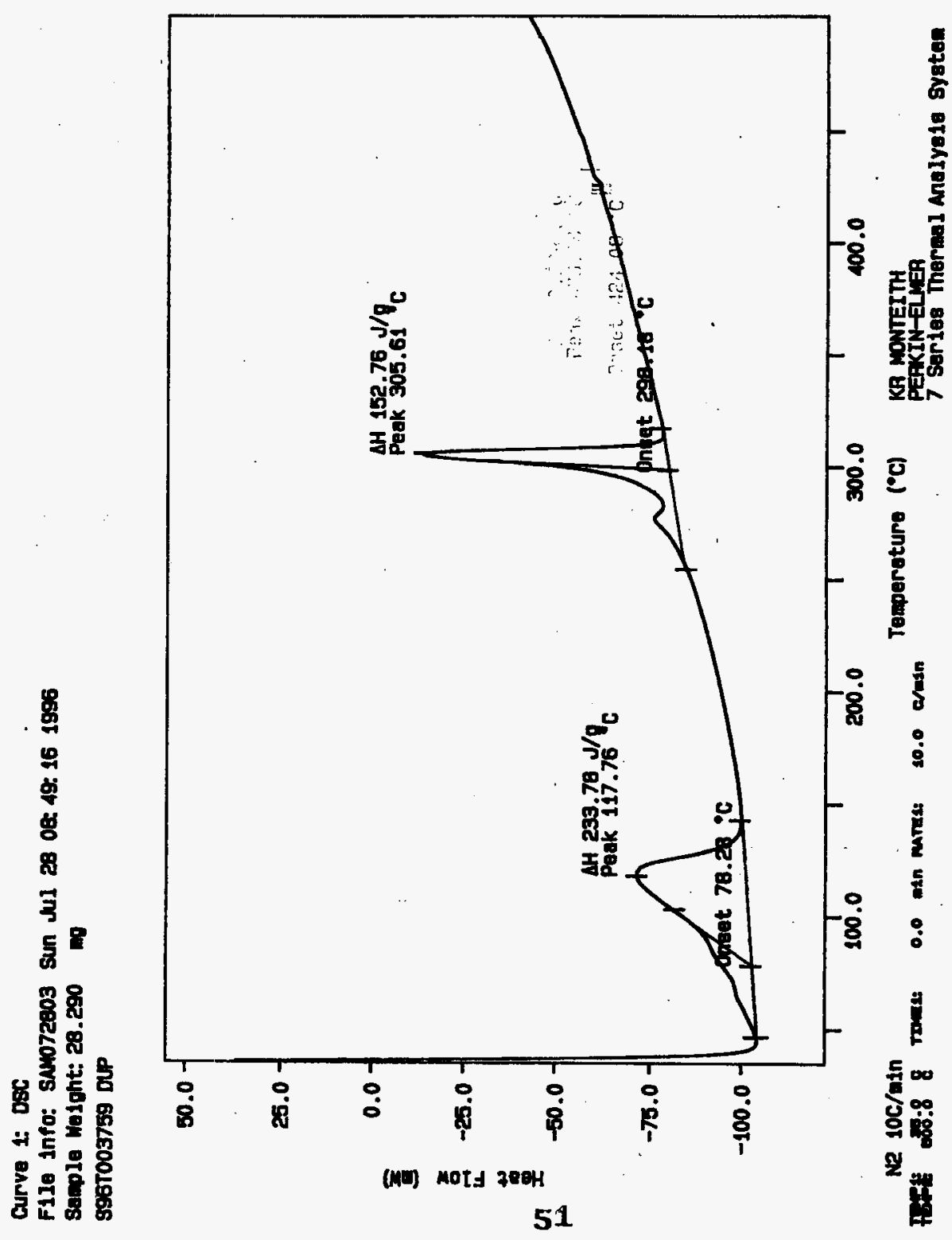




\section{LABCORE Data Entry Template for Worklist\#}

Analyst:

Instrument: DSCO I

Book $\# 2 N M A B$

Method: LA-514-113 Rev/Mod

Worklist Comment: S-109 DSC, RUN UNDER N2. RCJ

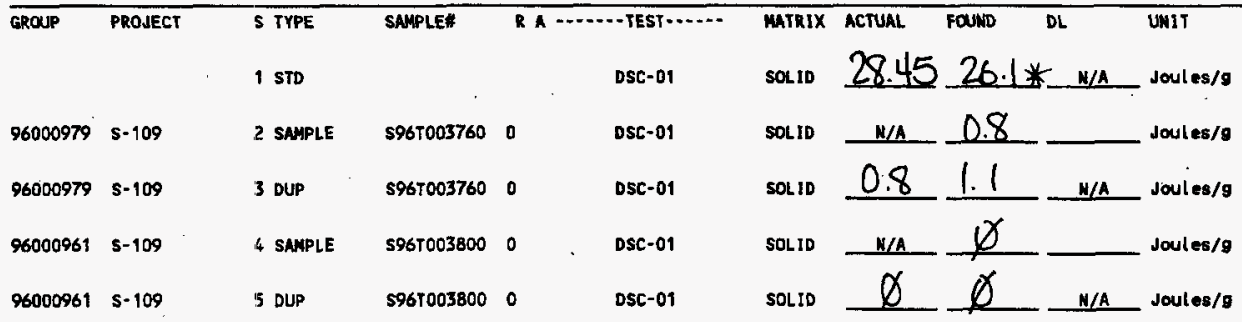

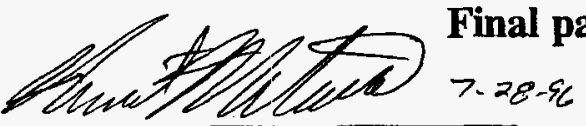

Analyst Signature Date Verified/Validated by Blandina Valenizuela
11174

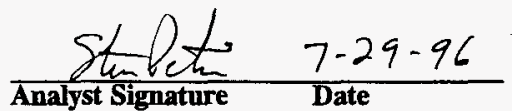

Data Entry Comments:

Units shown for $Q C$ (SPK \& STD) may not reflect the actual wnits. $D L=$ Detection Limit, $S=$ Worklist Slot Number, $R=$ Replicate Number, $A=$ Aliquot Code. 


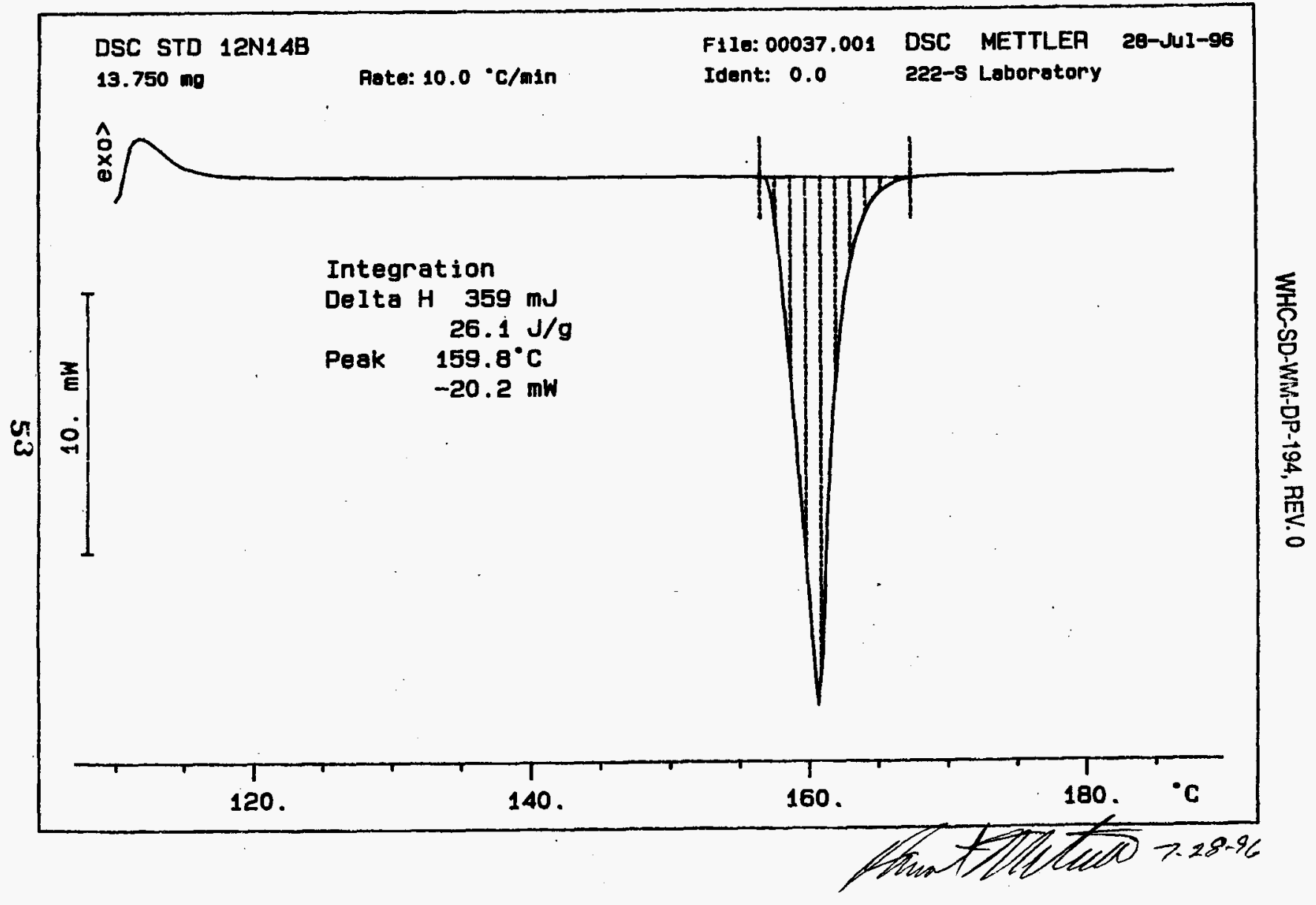




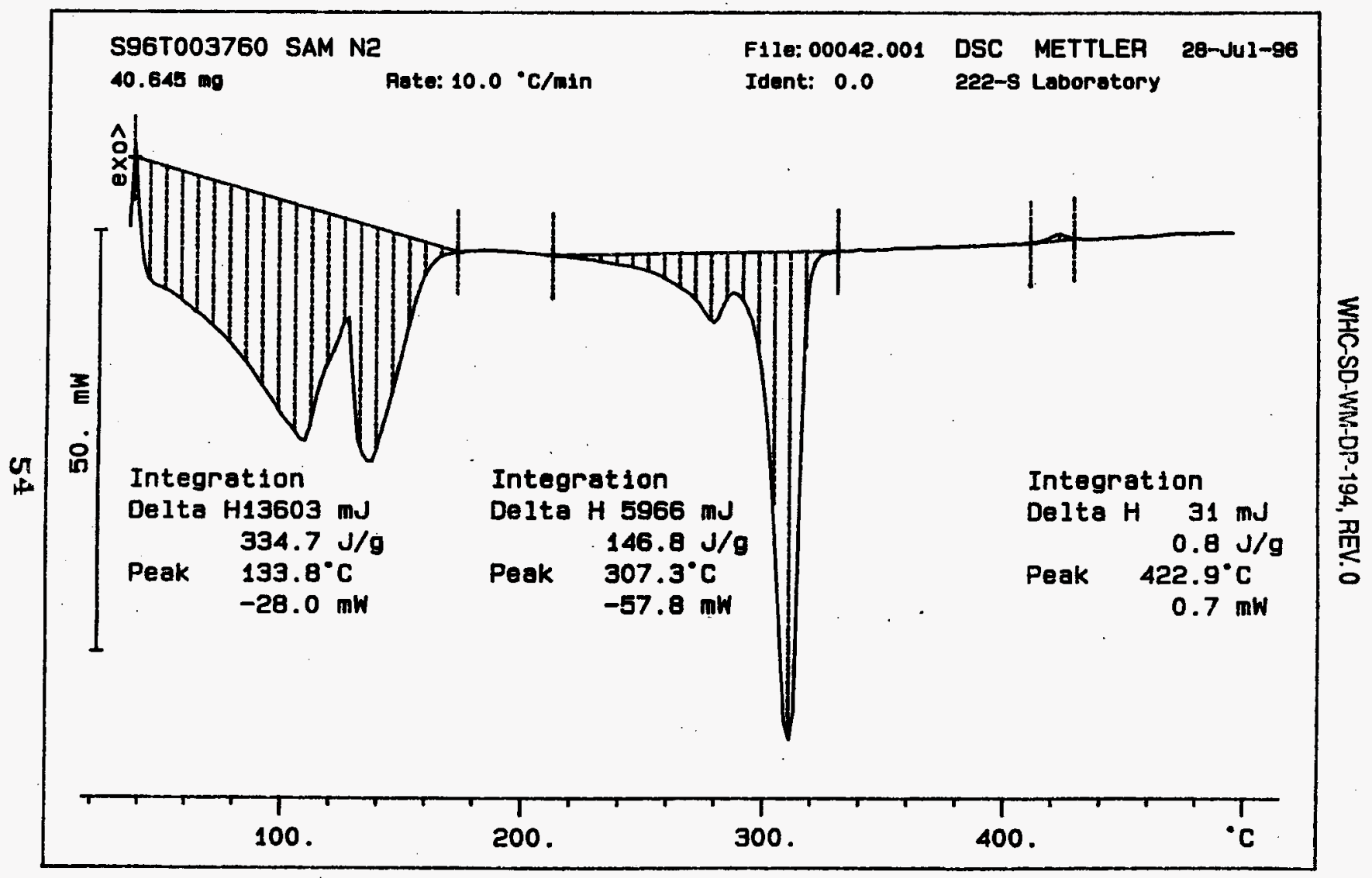




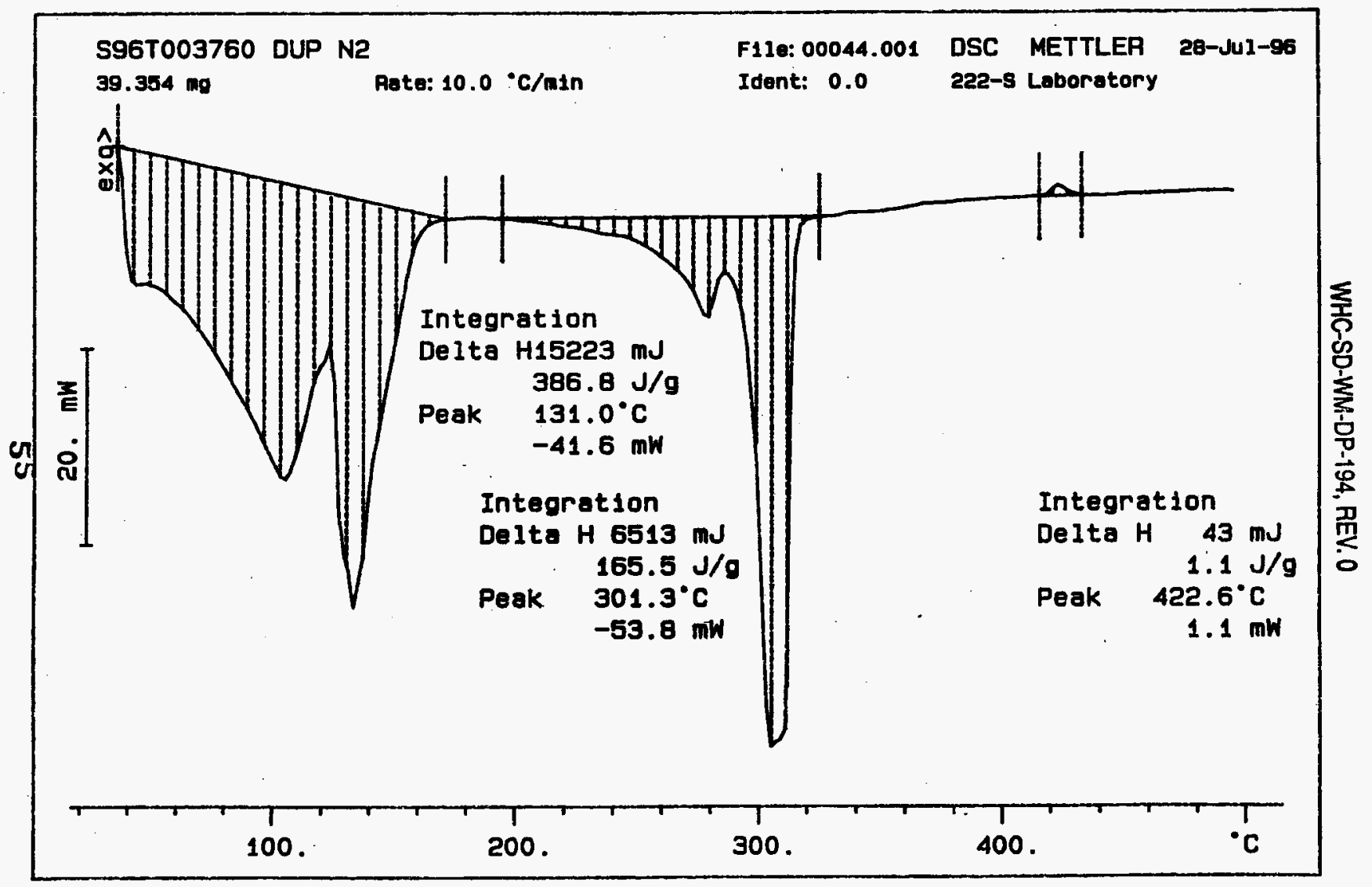




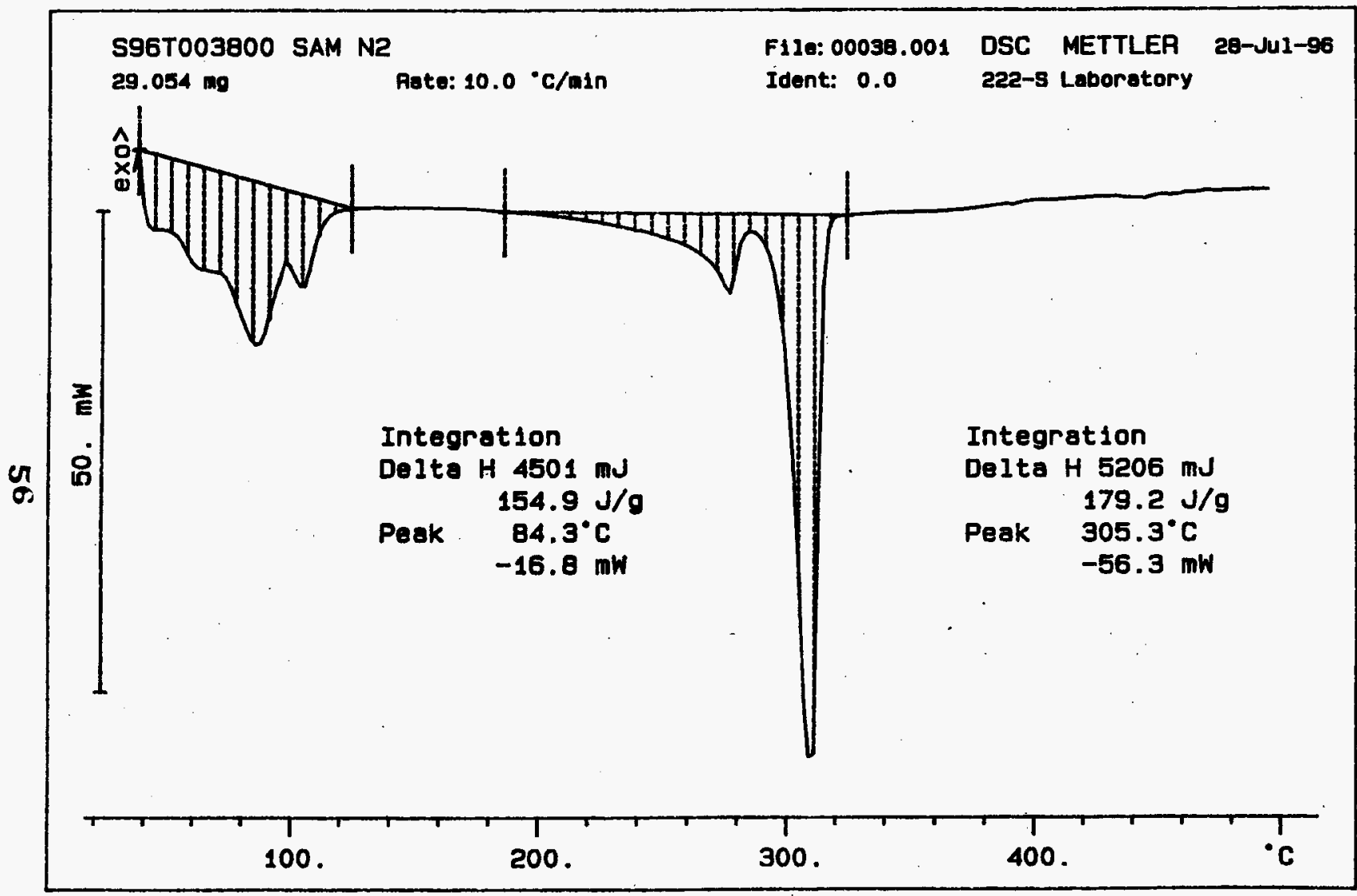

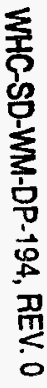




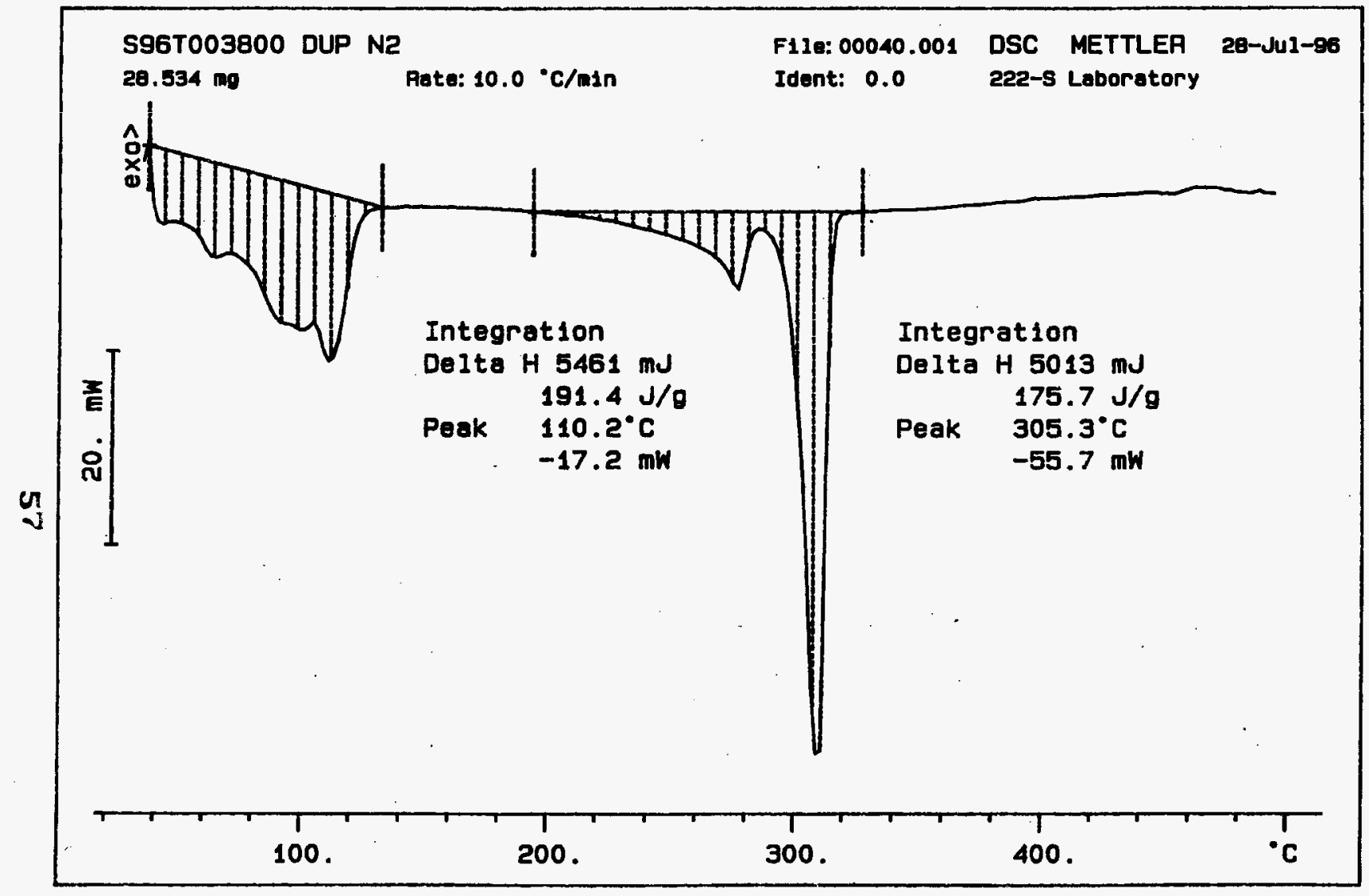




\section{LABCORE Data Entry Template for Worklist\#}

Analyst: $\quad$ KRM $\quad$ Instrument: DSCO 1 Book \#12N14. B

Method: LA-514-113 Rev/Mod C-1

Worklist Comment: S-109 DSC, RUN UNDER N2. RCJ

\begin{tabular}{|c|c|c|c|c|c|c|c|c|c|c|}
\hline \multirow[t]{2}{*}{$\overline{\text { GROUP }}$} & \multirow[t]{2}{*}{ PROJECT } & S TYPE & SAMPLE\# & \multicolumn{2}{|c|}{ R A $\cdots \cdots$....-TEST $\cdots$} & MATRIX & ACTUAL & FOUND & & UNIT \\
\hline & & 1 STD & & & DSC-01 & SOLID & 28.45 & $6.2 *$ & $N / A$ & Joul es/g \\
\hline 96000961 & s-109 & 2 SAMPLE & & 0 & DSC -01 & SOLID & H/A & $\varnothing$ & & Joules/g \\
\hline 96000964 & s-109 & 3 DUP & & & DSC-01 & SOLID & 0 & $\phi$ & H/A & Joules $/ 8$ \\
\hline 96000961 & s-109 & 4 SAMPLE & 5967003839 & 0 & DSE-01 & SOLID & N/A & $\varnothing$ & & Joules/g \\
\hline 96000961 & $s-109$ & 5 DUP & 5967003839 & 0 & DSC-01 & SOLID & $\varnothing$ & $\phi$ & $N / A$ & Joules/g \\
\hline
\end{tabular}

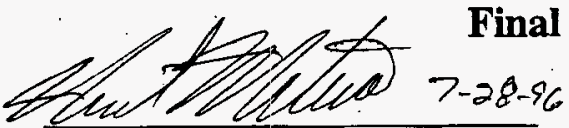

Analyst Sigmature

\section{Final page for worklist \#}

11175

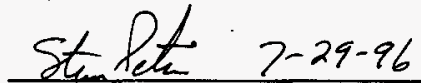
Date

$$
\begin{aligned}
& \text { Verified/Vacidatealy } \\
& \text { Blandina } \\
& \text { Valenzuela } \\
& \text { 7-31.96 }
\end{aligned}
$$




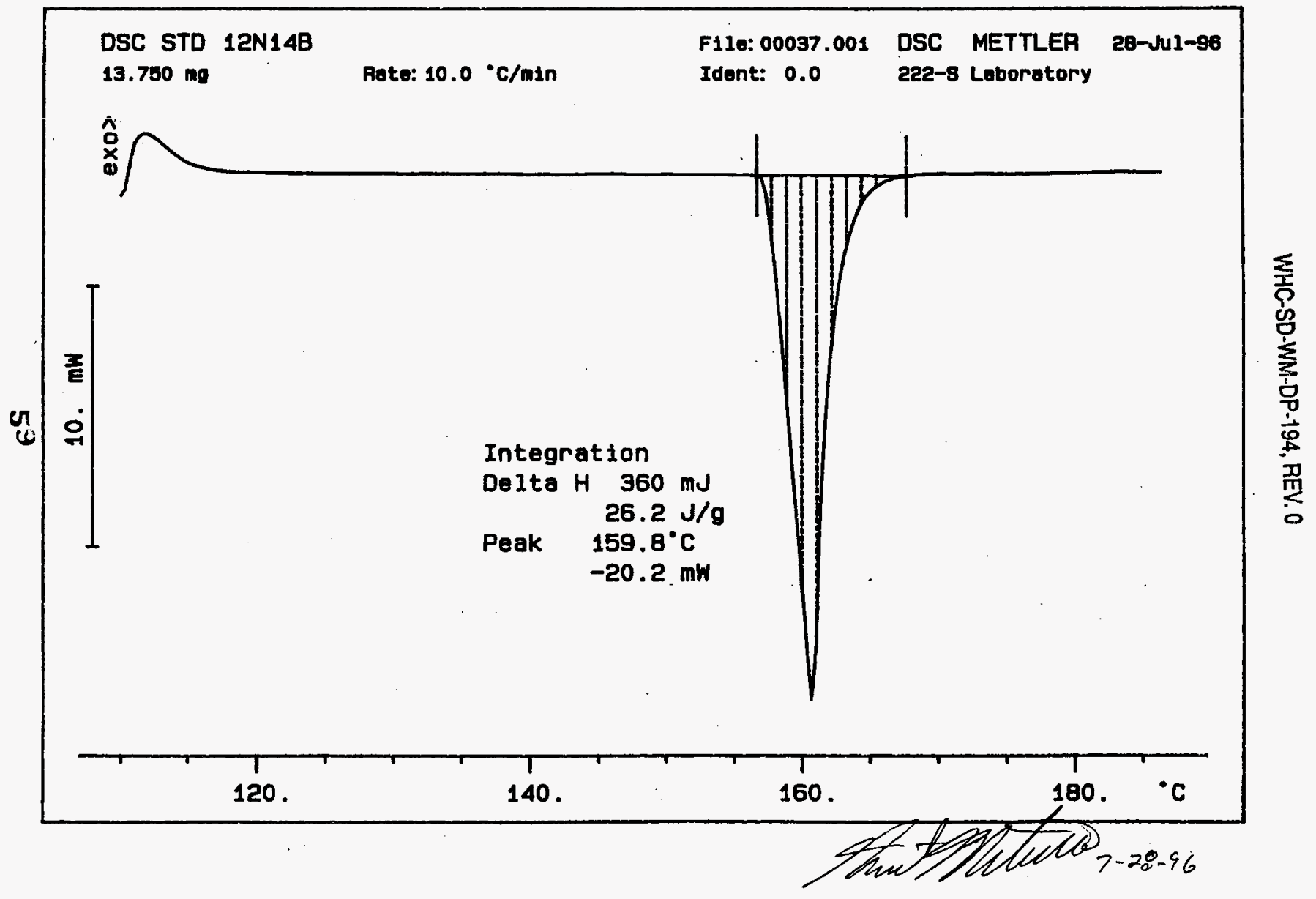




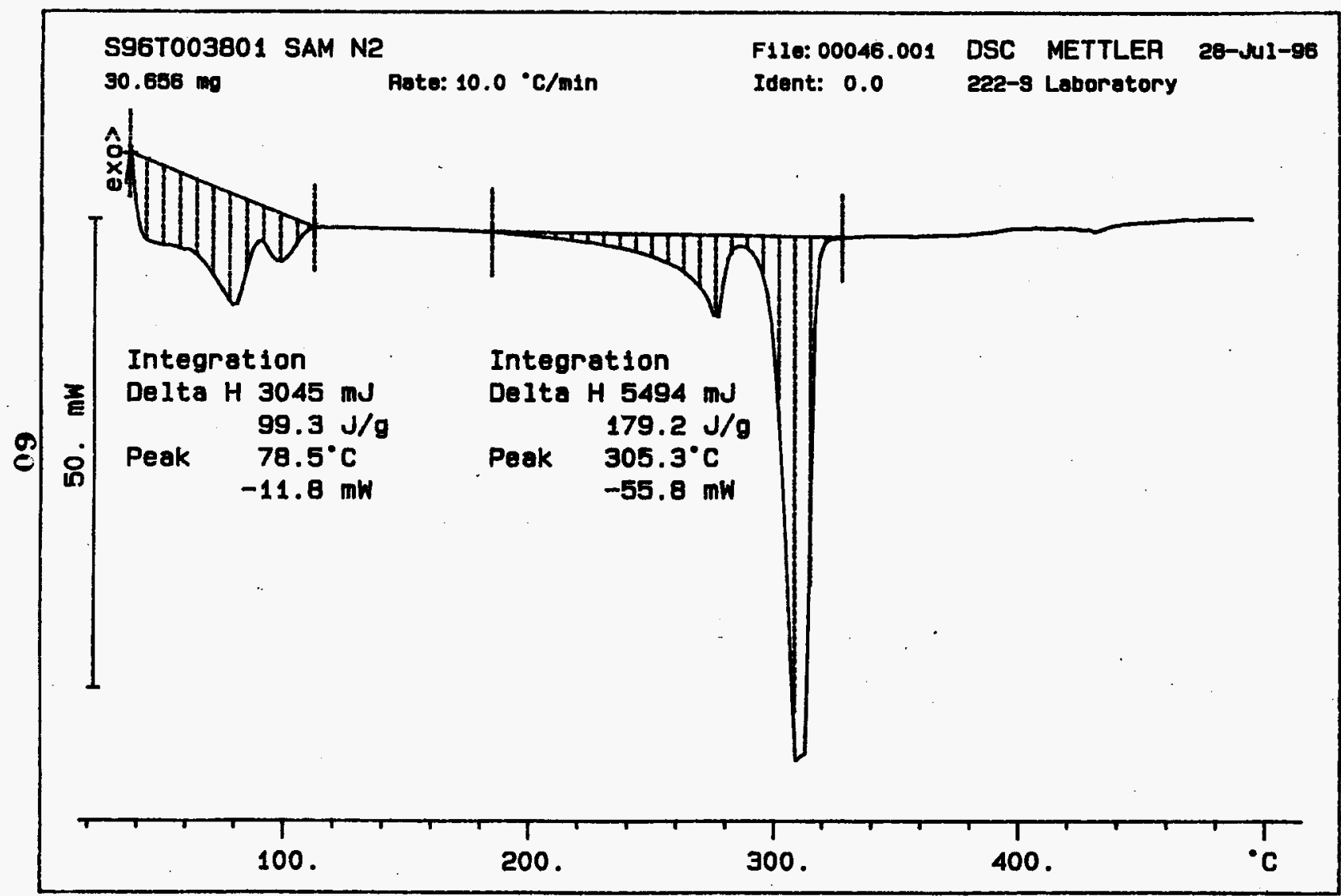




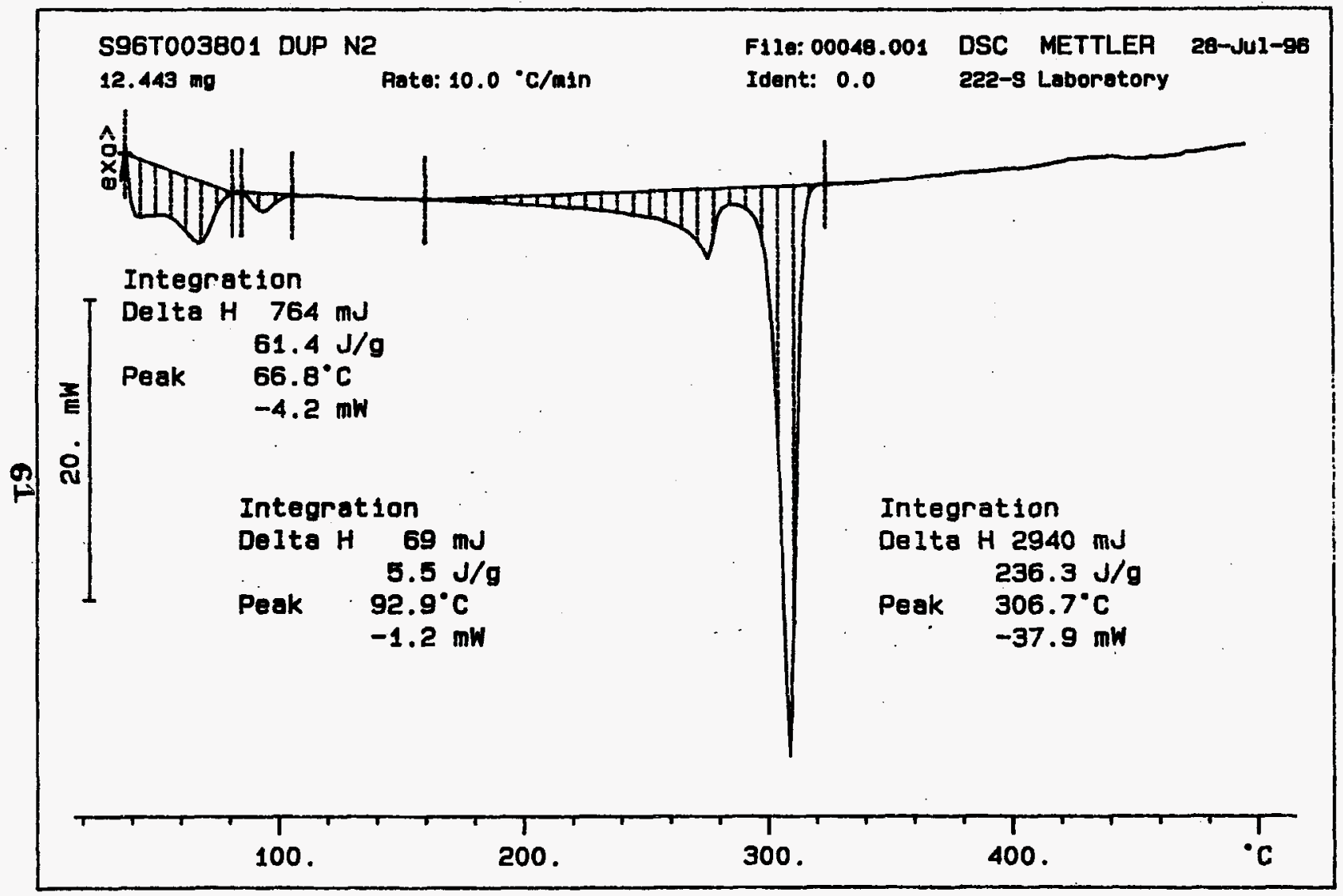




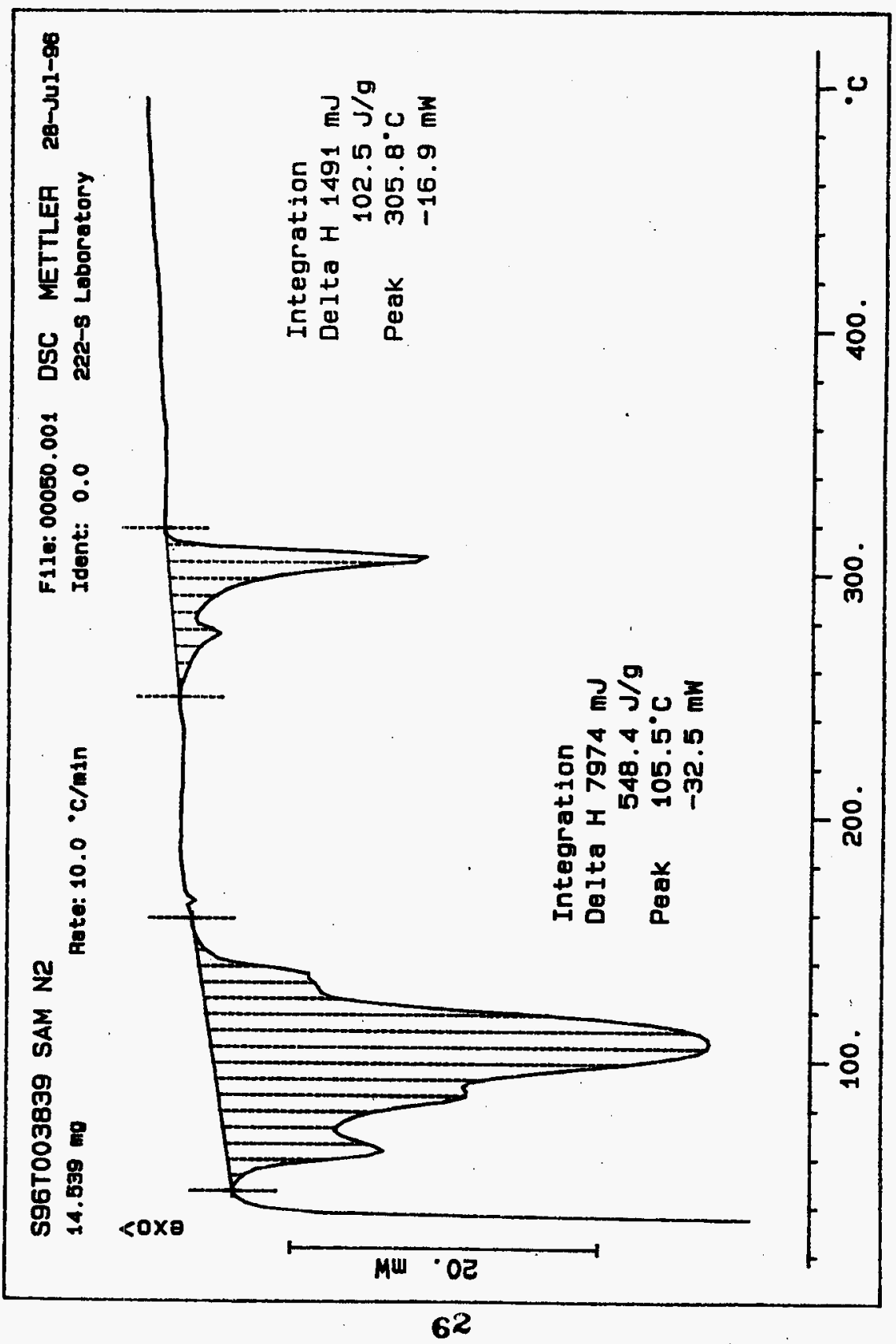




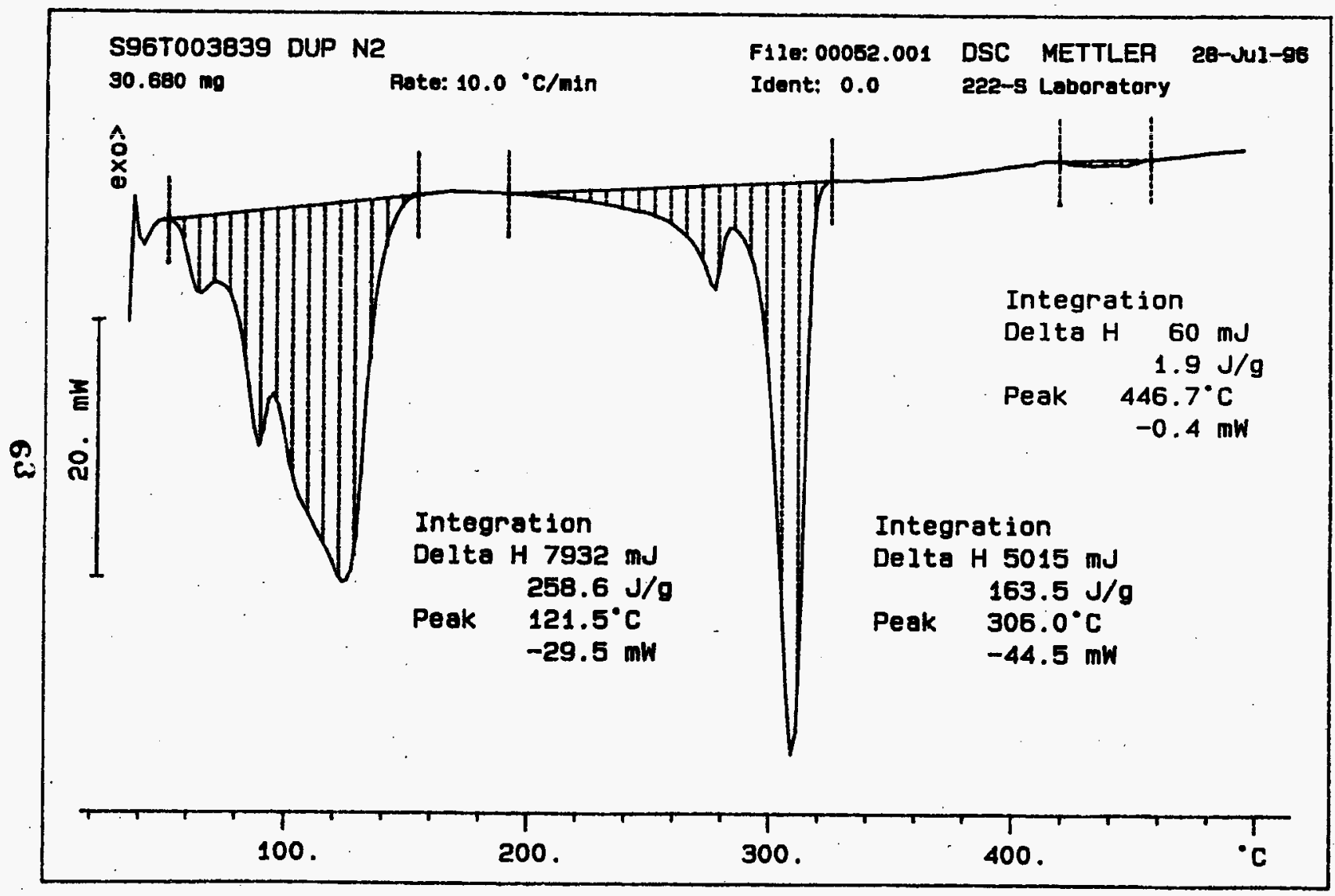




\section{LABCORE Data Entry Template for Worklist\#}

Analyst: $\quad$ KRM Instrument: DSCO $3 \quad$ Book \#12N14B

Method: LA-514-114 Rev/Mod C-I

Worklist Comment: S-109 DSC, RUN UNDER N2. RCJ

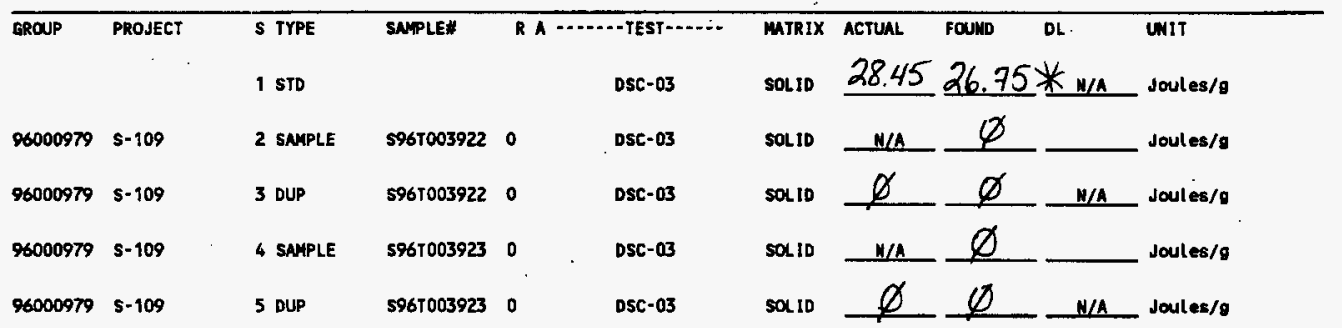

\section{Final page for worklist \# 11176}
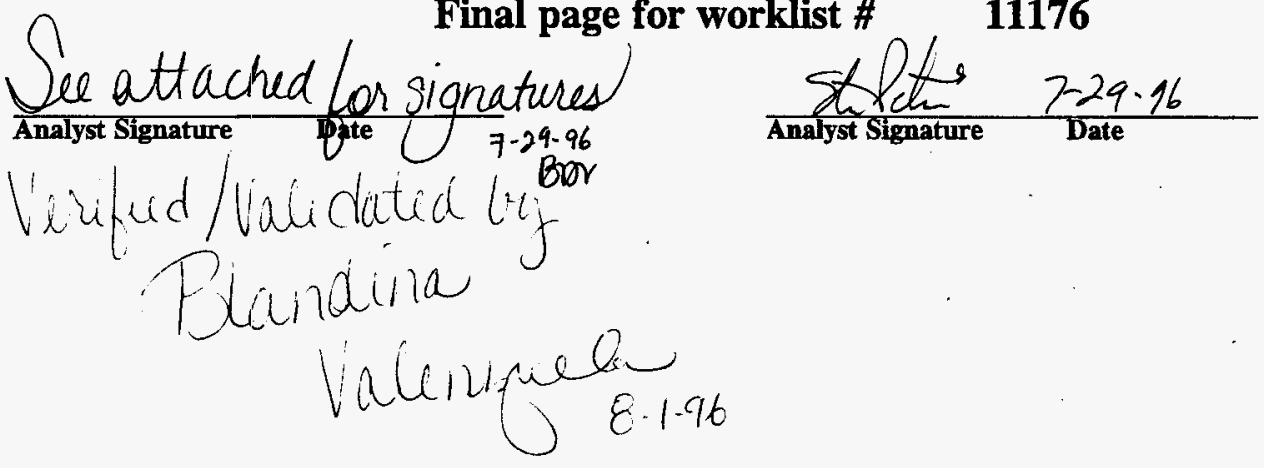

Data Entry Comments:

Units shoum for QC (SPK \& STD) may not reflect the actual units. $D L=$ Detection Limit, $S=$ Worklist Slot Number, $R=$ Replicate Nimber, $A=$ Aliquot Code. 


\section{LABCORE Data Entry Template for Worklist\#}

Analyst: KKin Instrument: DSC0 3 Book \# 12 N $14 \mathrm{~A}$

Method: LA-514-113 Rev/Mod $\mathrm{C}$

Worklist Comment: S-109 DSC, RUN UNDER N2. RCJ

\begin{tabular}{|c|c|c|c|c|c|c|c|c|c|c|c|}
\hline \multirow[t]{2}{*}{ GROUP } & PROJECT & \$ TYPE & SAMPLE: & R A - & -........ TEST $-\ldots$ & MATRIX & ACTUAL & Fouid & DL & UNIT & . \\
\hline & & 1 sto & & & DSC-01 & SOLID & & & $N / A$ & Joules/9 & \\
\hline 96000979 & $s-108$ & Z: SAMPLE & & & DSC-01 & SOLID & W/A & & & Joul es/g & . \\
\hline 96000979 & $s-109$ & ; DuP & & & DsC-01 & SOLID & & & $N / A$ & Joul es/g & \\
\hline 96000979 & $s-109$ & 4. SAMPLE & s96T003923 & 0 & DSC-01 & SOLID & $N / 4$ & & & Joules/g & \\
\hline 96000979 & $s-109$ & 5 DUP & \$96T003923 & 0 & DSC-01 & SOLID & & & N/A & Joules/g & \\
\hline
\end{tabular}

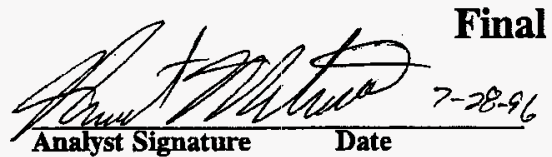

Analyst Signature Date

\section{Final page for worklist \# 11176}

\section{Analyst Signature Date}

DSC-03 Instrument was used.

Data Entry Comments:

Units shown for $Q C$ (SPK. \& STD) may not reflect the actual units. $D L=$ Detection Limit, $S=$ Worklist Slot Number, $R=$ Replicate Number, $A=$ Aliquot Code. 
Cupve 1: 0sc

File info: IND072e01 sun w1 20 04:50:23 1996

Somple Weight: 13.750 an

12N14-8 INDIUN AT 10C/MIN

OGNATURE BELOW FEPRESENTS CHEMICAL TECHNOLOGIST/CHEMIST THAT COMPLETED/VERIFIED THE CALIBRATION/ANALYSIS ON PAGES 66 TO 70.

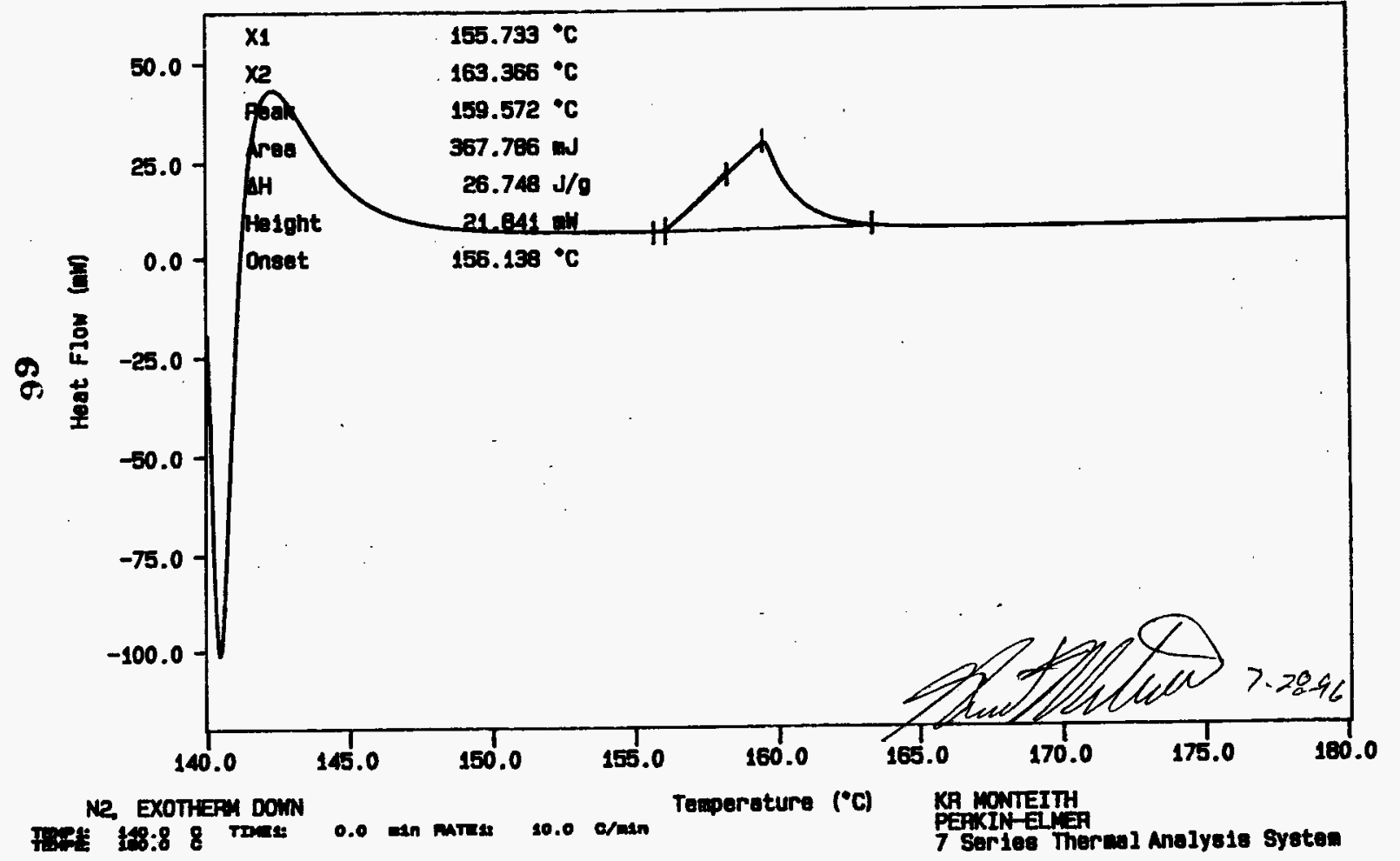


WHC-SD-WM-DP-194, REV. 0

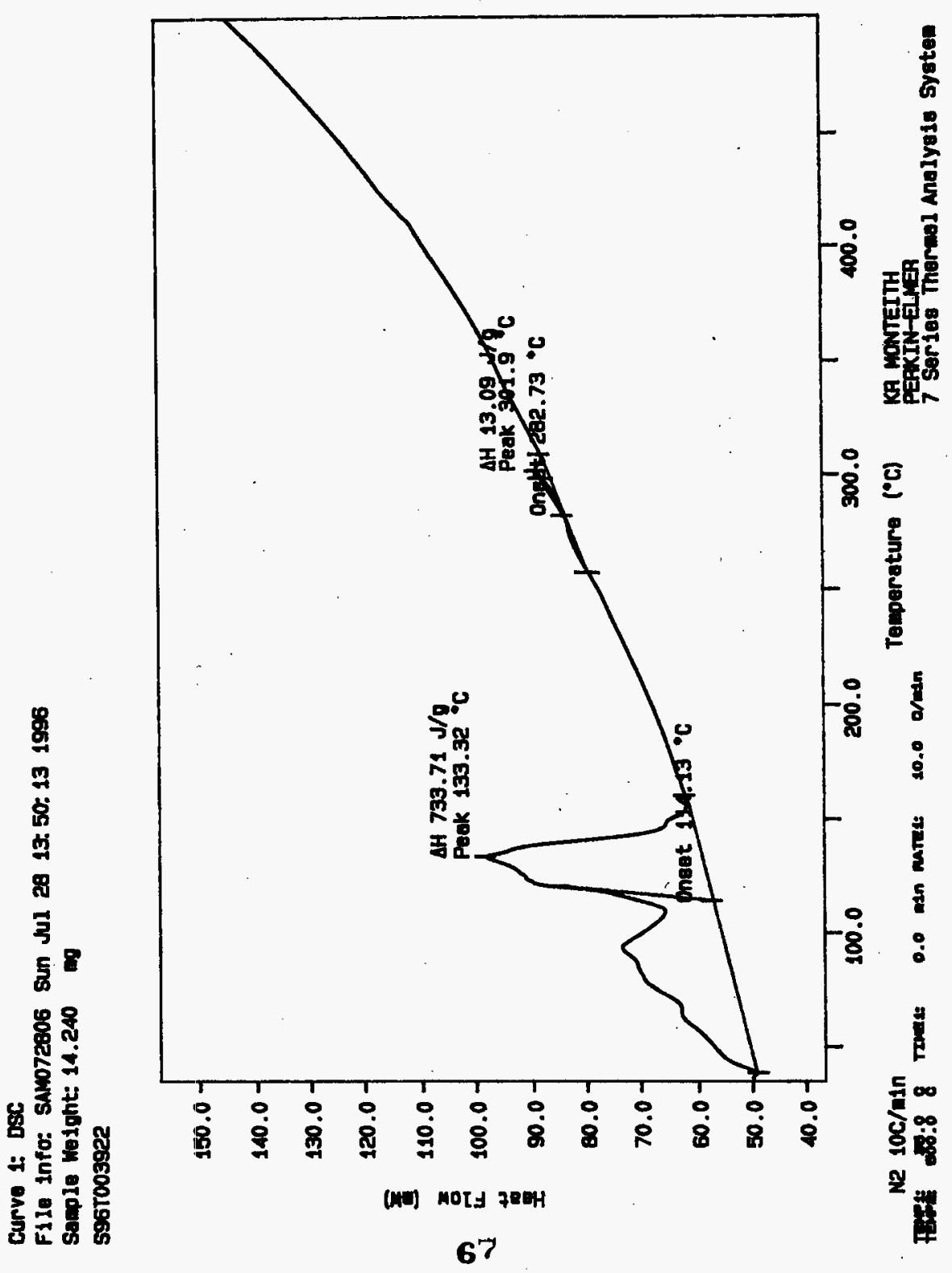


WWHC-SD-WM-DP-194, REV. O

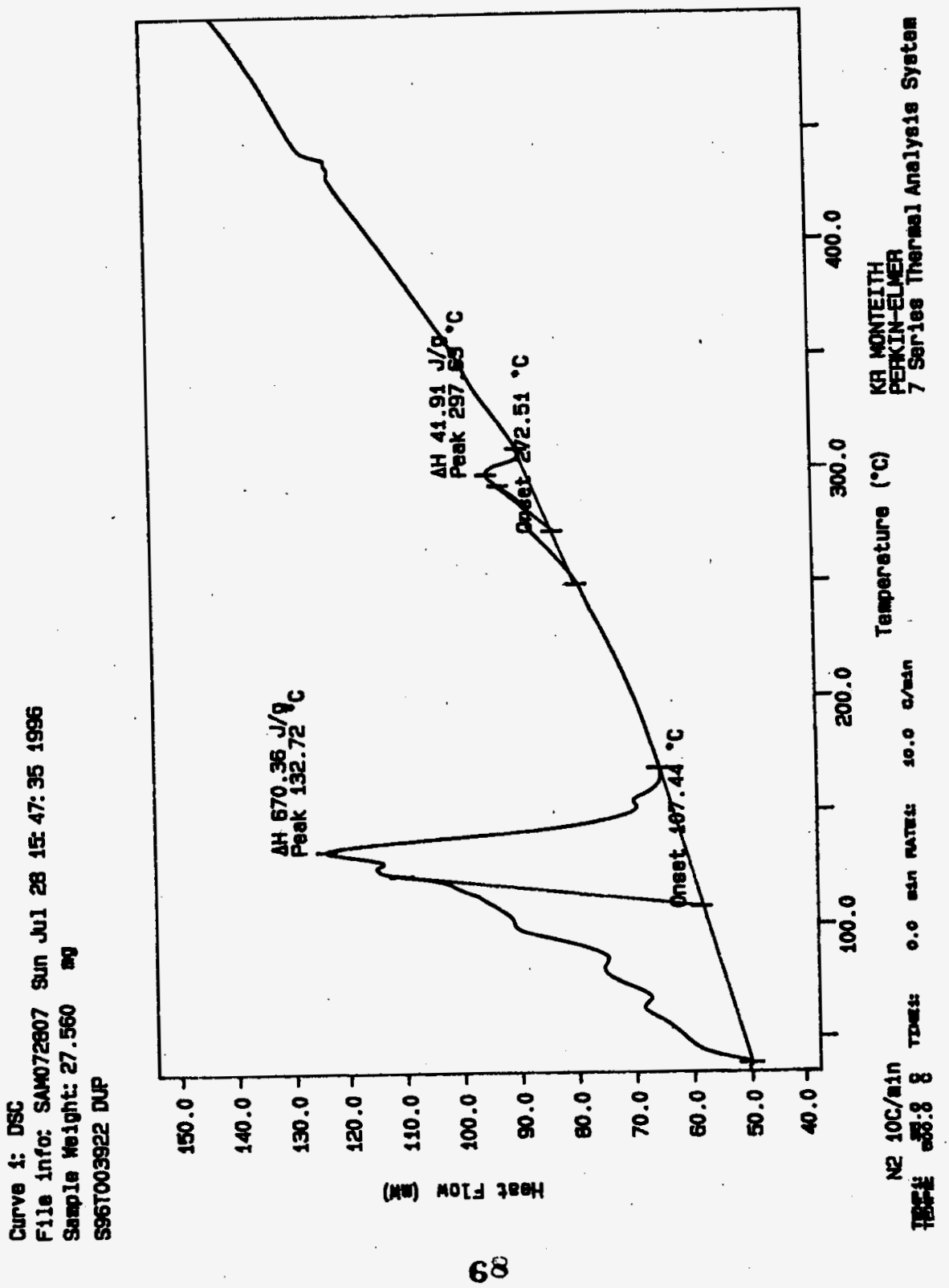


Curve 1: DSC

File Info: SANo72808 Sun. Jul 28 19: 14: 401996

Sanple We1ght: 34.360

S96T003923 SAM

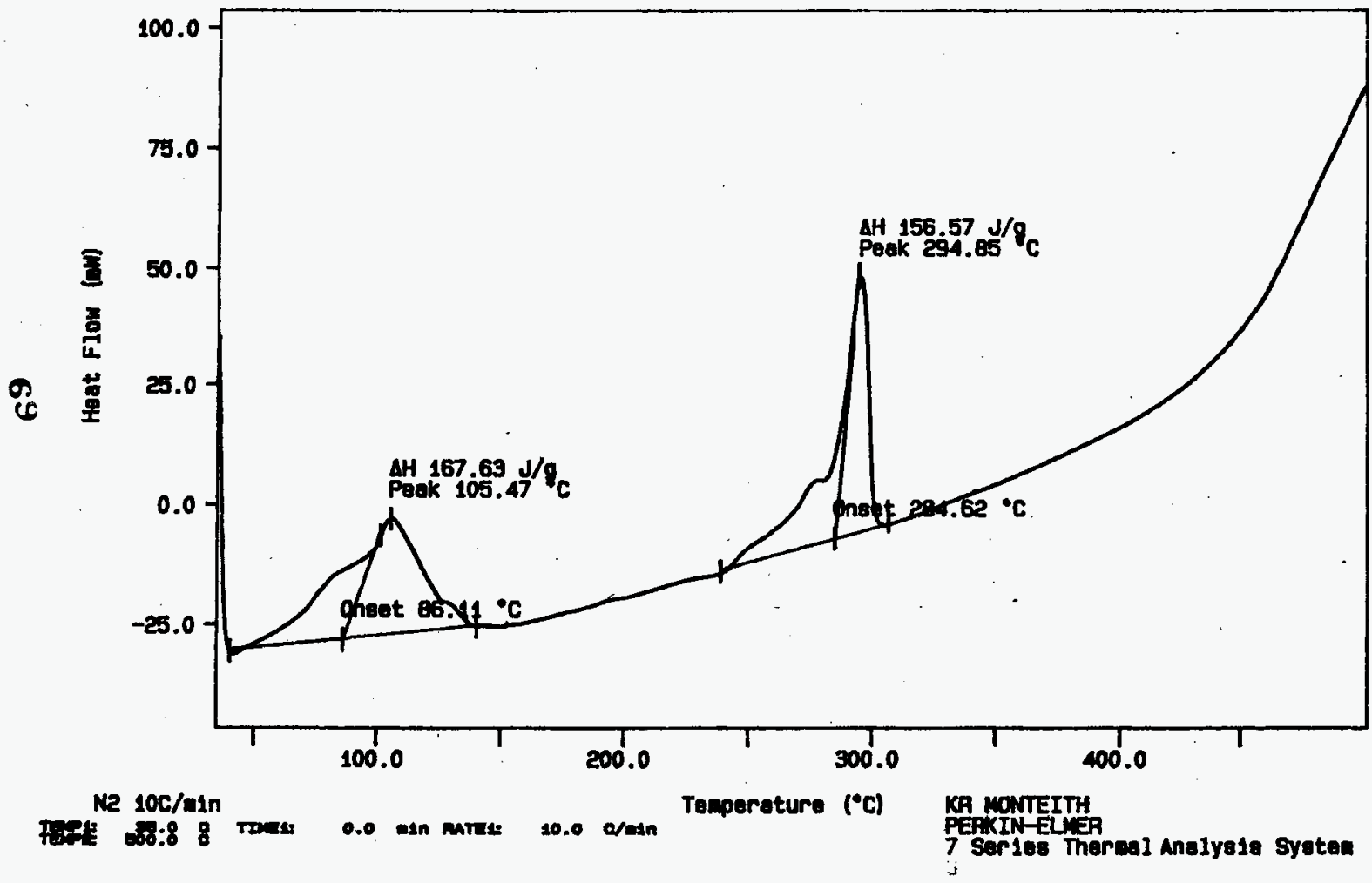


WHA-SD-WM-DP-194, REV. O

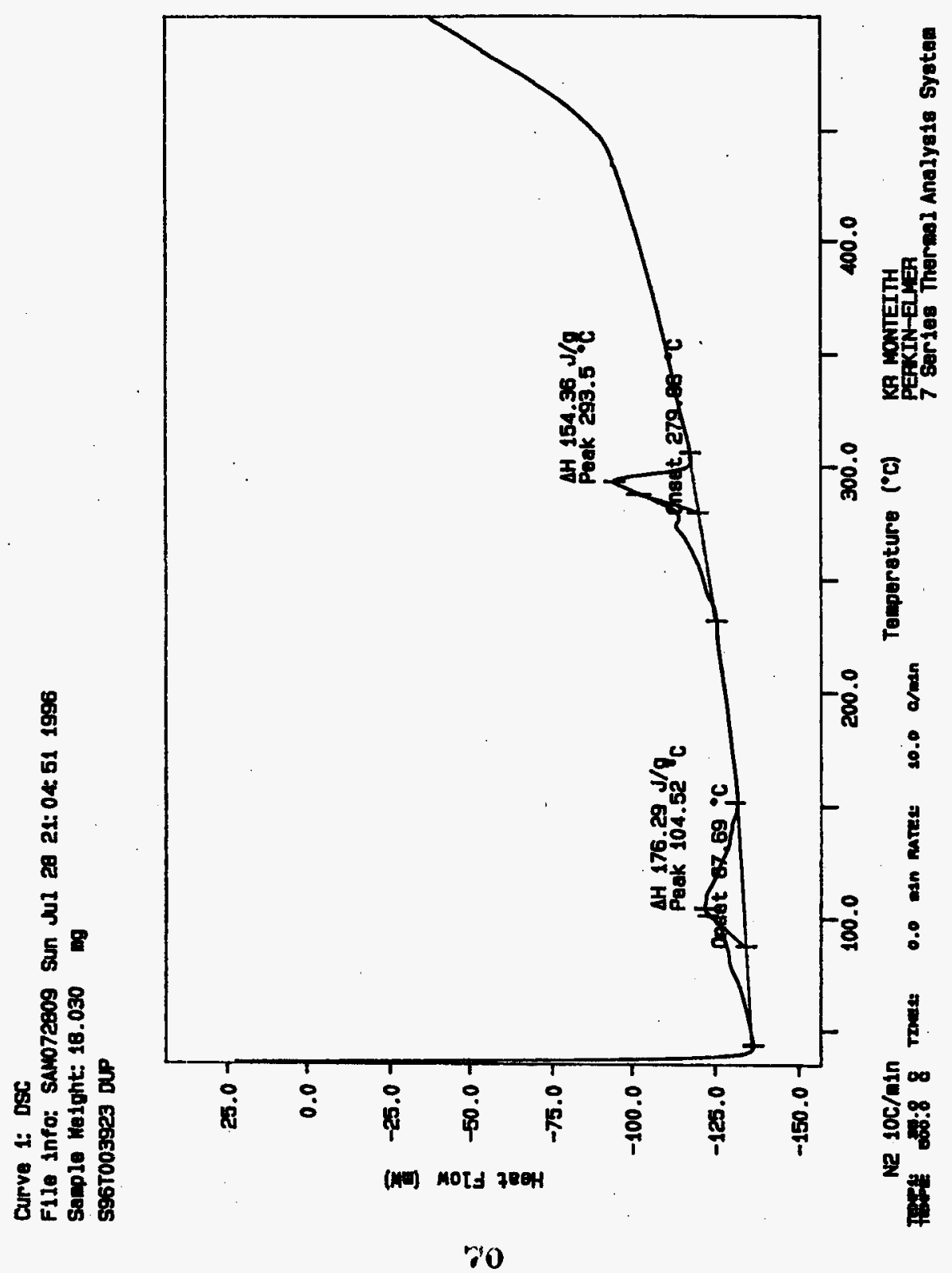




\section{LABCORE Data Entry Template for Worklist\#}

Analyst: $\quad A D P \quad$ Instrument: $D S C 03$ Book \#12N14B

Method: LA-514-114 Rev/Mod C-1

Worklist Comment: S-109 DSC, RUN UNDER N2. RCJ

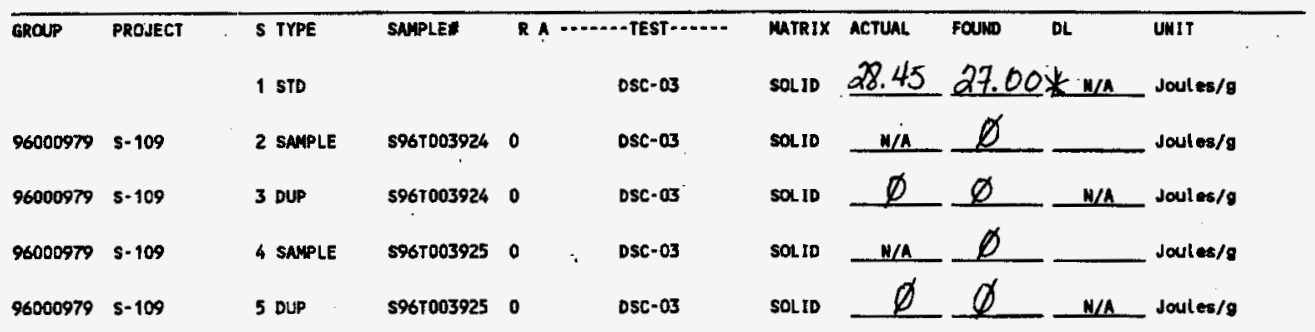

\section{Final page for worklist \#}

Ke attached for signatures)

Verified/Validatea by Bor

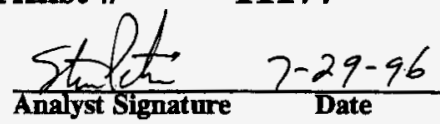

11177

Blandina

Valenzuela

Data Entry Comments:

Units shown for QC (SP.K \& STD) may not reflect the actual units. $D L=$ Detection Limit, $S=$ Worklist Slot Number,

$\boldsymbol{R}=$ Replicate Nimber, $A=$ Aliquot Code. 


Analyst: $A P$ Instrument: DSCO Book\#12N14B

Method: LA-514-113 Rev/Mod -1

Worklist Comment: S-109 DSC, RUN UNDER N2. RCJ

\begin{tabular}{|c|c|c|c|c|c|c|c|c|c|c|}
\hline \multirow[t]{2}{*}{$\overline{\text { ERCUP. }}$} & PROJECT & S TYPE & SAMPLE* & $\mathbf{R}$ & A $\cdots-\ldots$ - TEST $\cdots \ldots$ & MATRIX & ACTUAL & FOUND & $\mathrm{DL}$ & UNIT \\
\hline & & 1 STO & & & DSC-01 & SOLID & & & $N / A$ & Joules/o \\
\hline 96000979 & $s-109$ & 2 SAMPLE & S96т003924 & 0 & DSC -01 & SOLID & $\omega / A$ & & & Joules/g \\
\hline 96000979 & $s-109$ & 3 DUP & \$961003924 & 0 & DSC-01 & SOLID & & & N/A. & Jout es/g \\
\hline 96000979 & $5-109$ & 4 SAMPLE & 5961003925 & 0 & DSc-01 & SOLID & $\omega / \mathbf{A}$ & & & Joules/g \\
\hline 96000979 & $s-109$ & 5 DUP & 5961003925 & 0 & DSC-01 & SOLID & & & $\mathbf{N} / \mathbf{A}$ & Sout es/9 \\
\hline
\end{tabular}

tof turuntor 78 7-28-96

\section{Final page for worklist \# 11177}

Analyst Signature Date

DSC.03 instrument

was used.

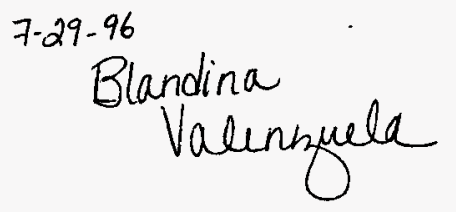

Data Entry Comments:

Units shown for QC (SPK \& STD) may not reflect the actual conits. $D L=$ Detection Limit, $S=$ Worklist Slot Number, $R=$ Replicate Number, $A=$ Aliguot Code. 
Curve 1: DSC

Flle info: IND072801 Sun Jul 28 04: 50: 231996

Sanple Weight: $13.750 \quad n g$

12N14-B INDIUM AT 10C/MIN

SIGNATUFE BEI OW AEPFESENTS CHEMICAL TECHNOLOGIST/CHEMIST THAT COMPLETEDIVERIFED THE CALIBRATIONIANALYSIS ON PAGES 73 TO 77

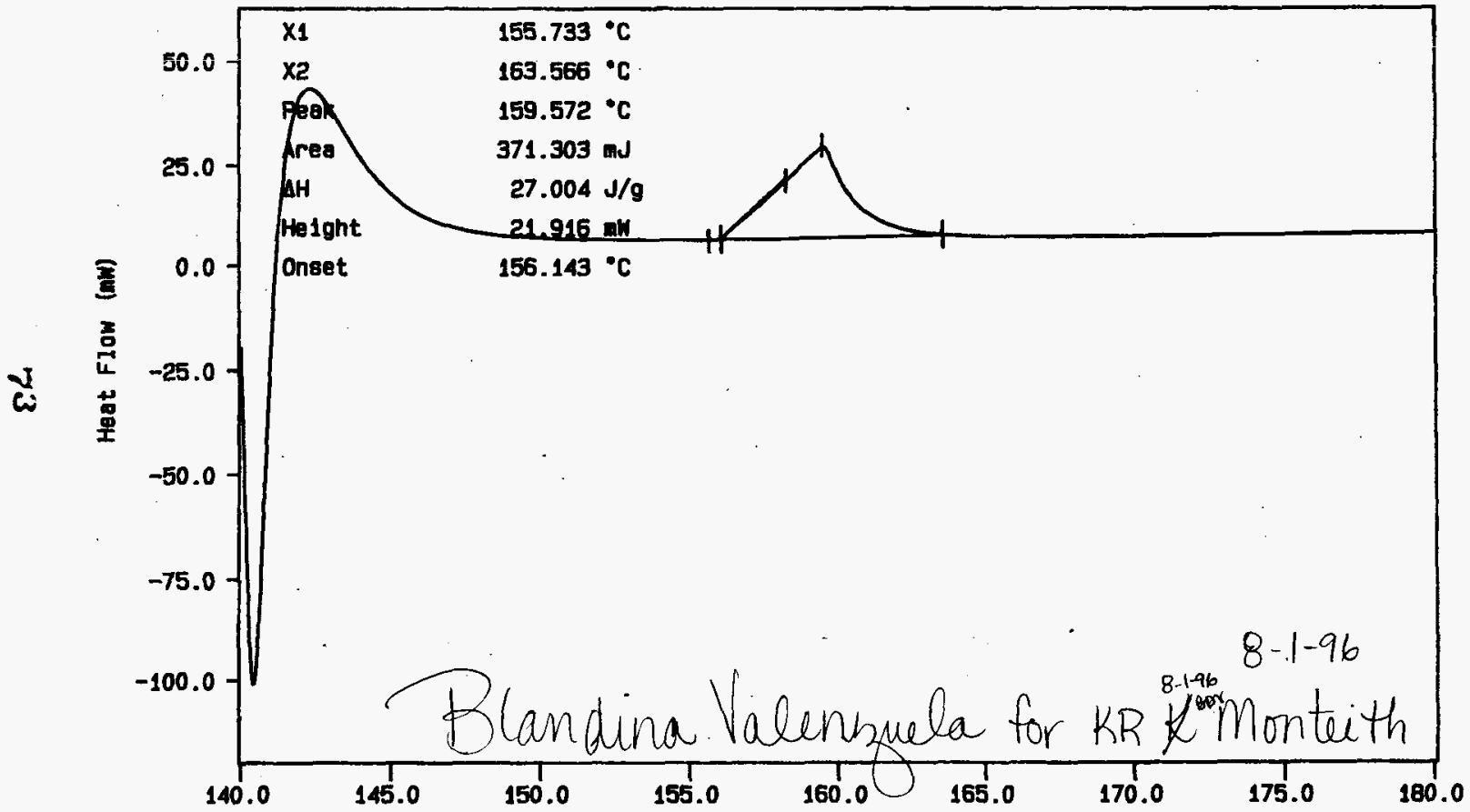

N2. EXOTHERM DOWN

Fapt: 140:8 \& Trues:

0.0 man antel: $10.0 \mathrm{c} / \mathrm{min}$

Temperature ('C) KR MONTEITH

7 Series Thermel Analyajs Systea

Mon Jul 29 05: 44: 421996 
WHC-SD-WM-DP-194, REV. 0

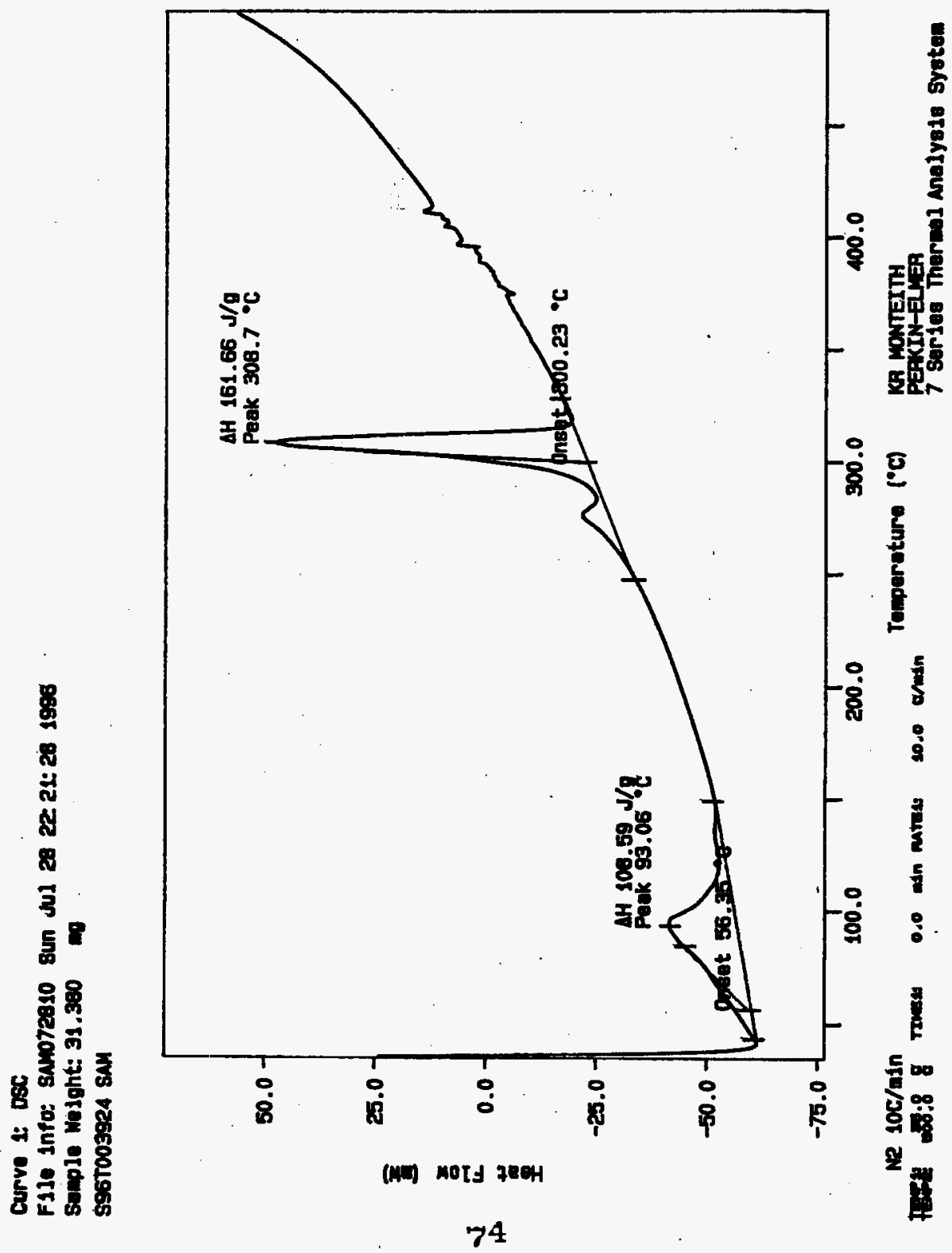


Curve 1: DaC

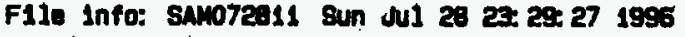

8anple Vadght: 36.030

sestoosge4 olp

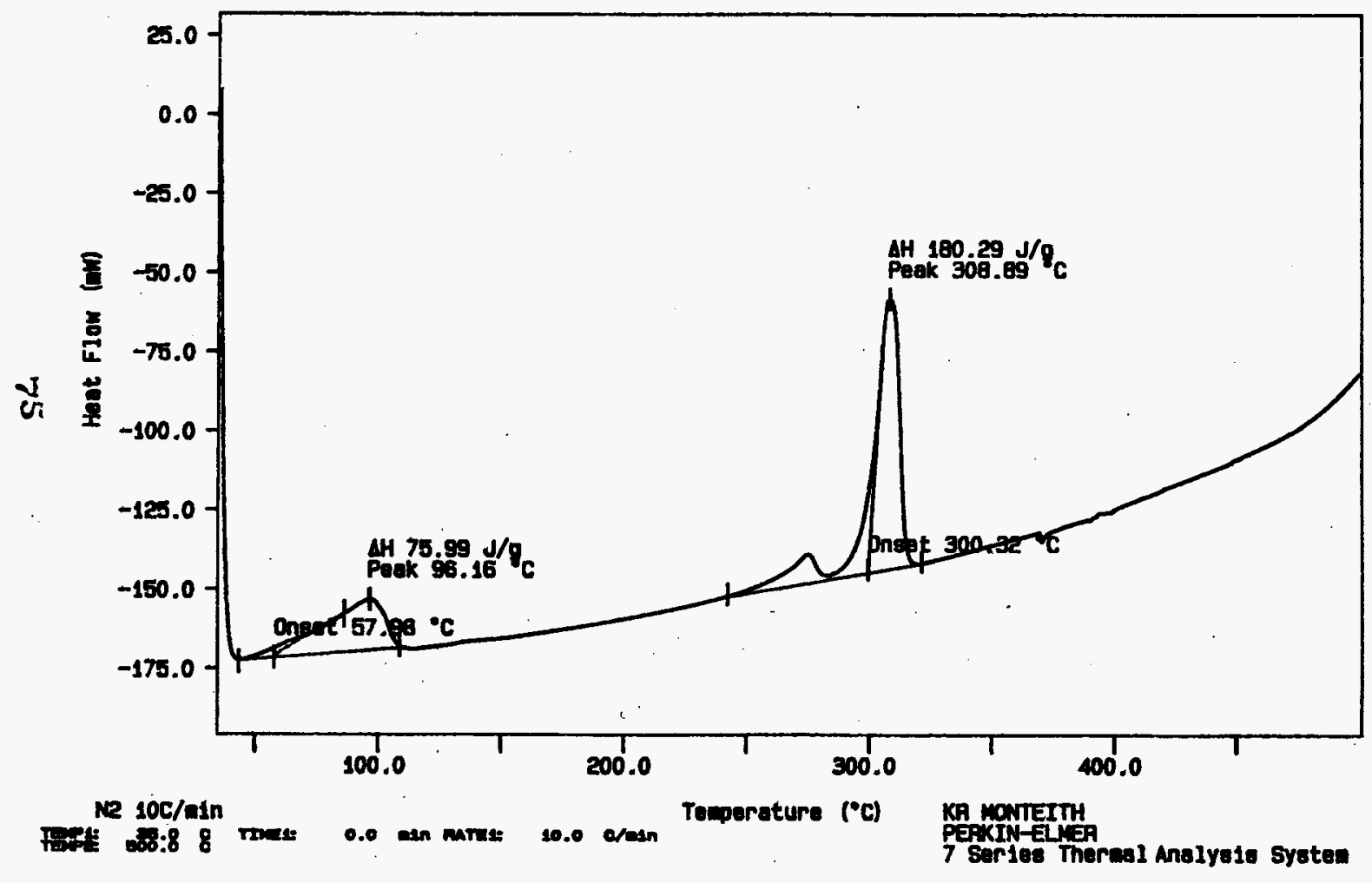


Curve 1: Dac

F110 info: SAmo72a12 kon wl 29 01: 39.541996

Sanple weight: 16.140

Sg6T003ges SAM

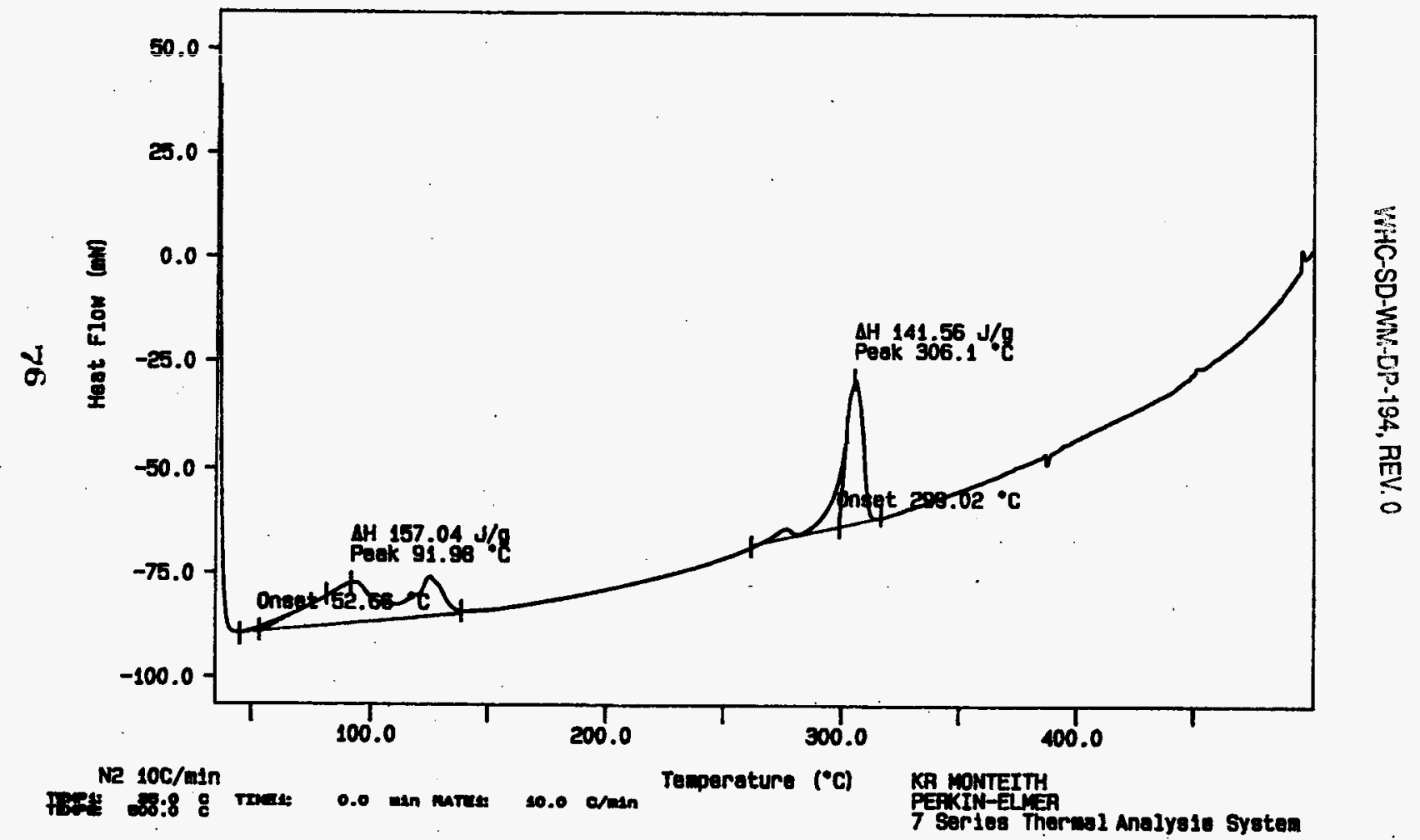




\section{Curve 1: DSC}

F1le info: SAM072813 Mon Ju1 29 02:57:08 1996

Sample Weight: $34.780 \mathrm{mg}$

5967003925 DUP

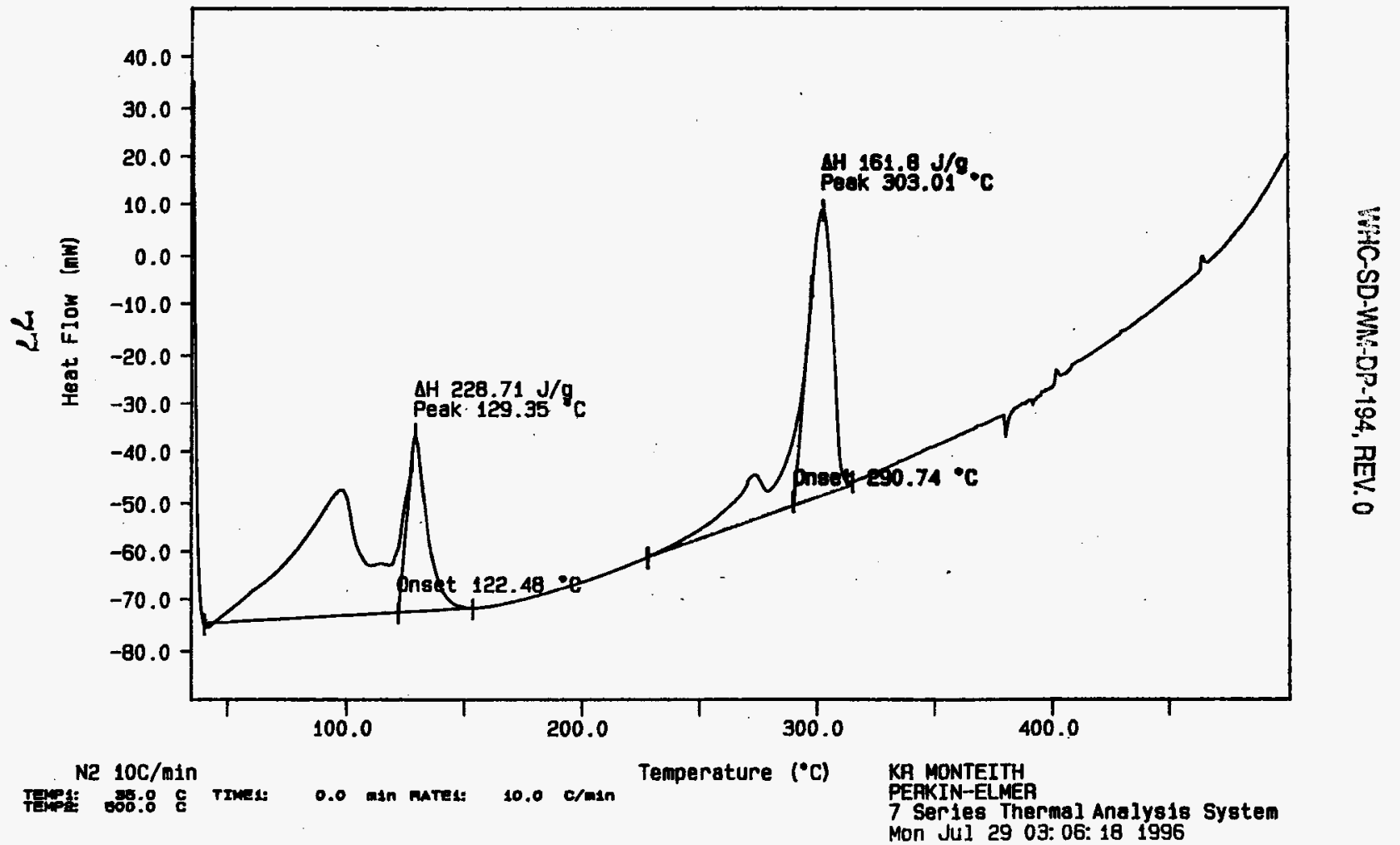


Analyst: HDD Instrument: DSCO $\perp$ Book \# $12 N / 4 B$

Method: LA-514-113 Rev/Mod $\mathrm{C}-{ }_{-}$

Worklist Comment: S-109 DSC, RUN UNDER N2. RCJ

\begin{tabular}{|c|c|c|c|c|c|c|c|c|c|c|}
\hline \multirow[t]{2}{*}{$\overline{\text { GROUP }}$} & \multirow[t]{2}{*}{ PROJECT } & \multirow{2}{*}{$\begin{array}{l}S \text { TYPE } \\
\text { STD }\end{array}$} & \multirow[t]{2}{*}{ SAMPLE\# } & \multicolumn{2}{|c|}{ R A $\cdots \cdots . . .-$ TEST - - . . } & \multirow{2}{*}{$\begin{array}{l}\text { MATRIX } \\
\text { SOLID }\end{array}$} & \multirow{2}{*}{$\begin{array}{l}\text { ACTUAL } \\
28.45 \\
\end{array}$} & \multirow{2}{*}{$\begin{array}{l}26.2 * \\
\end{array}$} & \multirow{2}{*}{ W/A } & \multirow{2}{*}{$\begin{array}{l}\text { UHIT } \\
\text { Joul es/g }\end{array}$} \\
\hline & & & & & DSC-01 & & & & & \\
\hline 96000979 & s-109 & 2 SAMPLE & \$965003926 & 0 & DSC-01 & SOLID & $N / A$ & 1.2 & & Joules/g \\
\hline 96000979 & s-109 & 3 oup & S96T003926 & 0 & Dse-01 & SOL10 & 1.2 & $\not \varnothing$ & N/A & Joules/g \\
\hline 96000979 & s-109 & 4 SAMPLE & s96r003927 & 0 & DSC.01 & SOLID & $\mathrm{N} / \mathrm{A}$ & $\varnothing$ & & Joul es/s \\
\hline 96000979 & s-109 & 5 DUP & 5967003927 & 0 & DSC-01 & SOL-10 & $\varnothing$ & 30.5 & N/A & Joules/g \\
\hline
\end{tabular}

Final page for worklist \# 11178
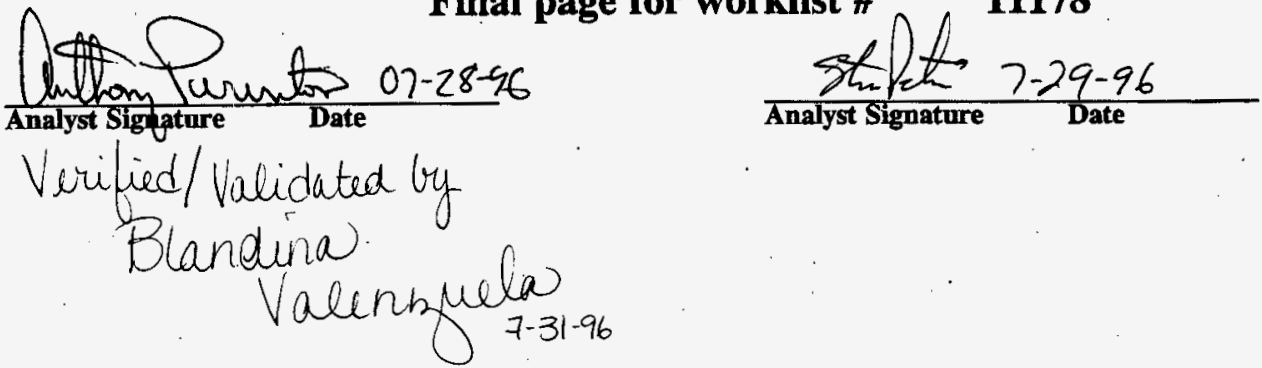

Data Entry Comments:

Units shown for QC (SPK \& STD) may not reflect the actual units. $D L=$ Detection Limit, $S=$ Wonklist Slot Number, $R=$ Replicate Number, $A=$ Aliguot Code. 


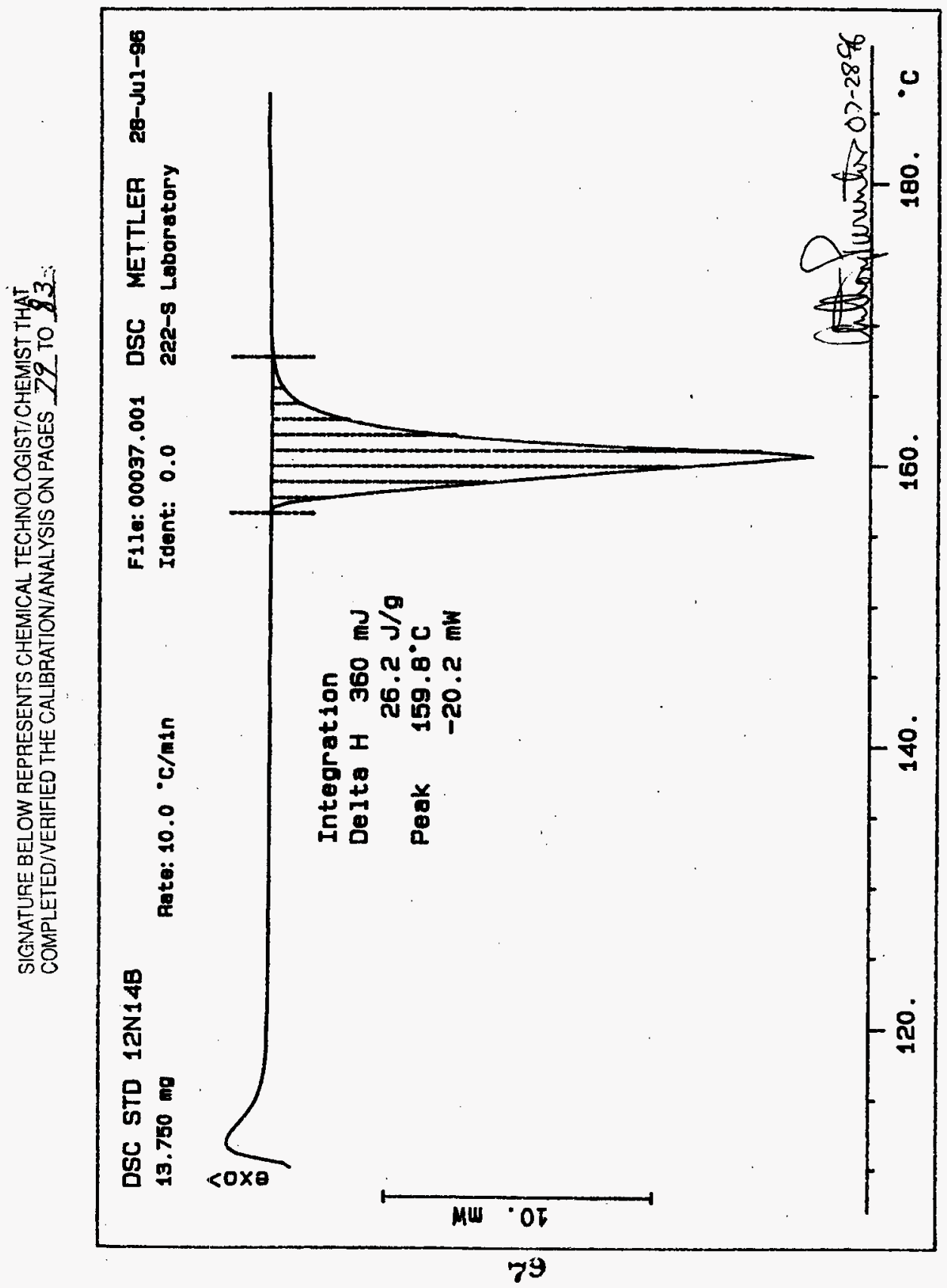




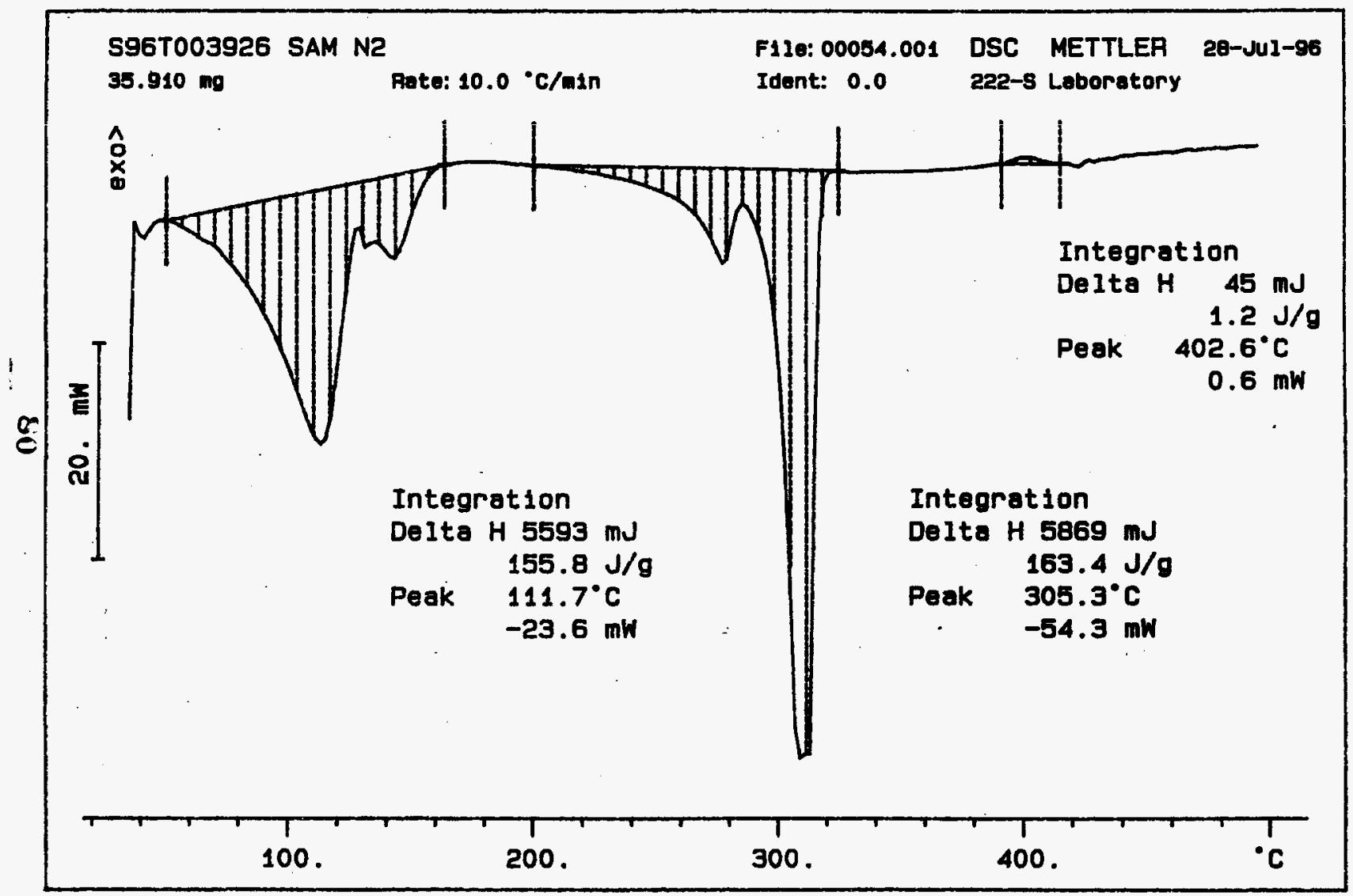




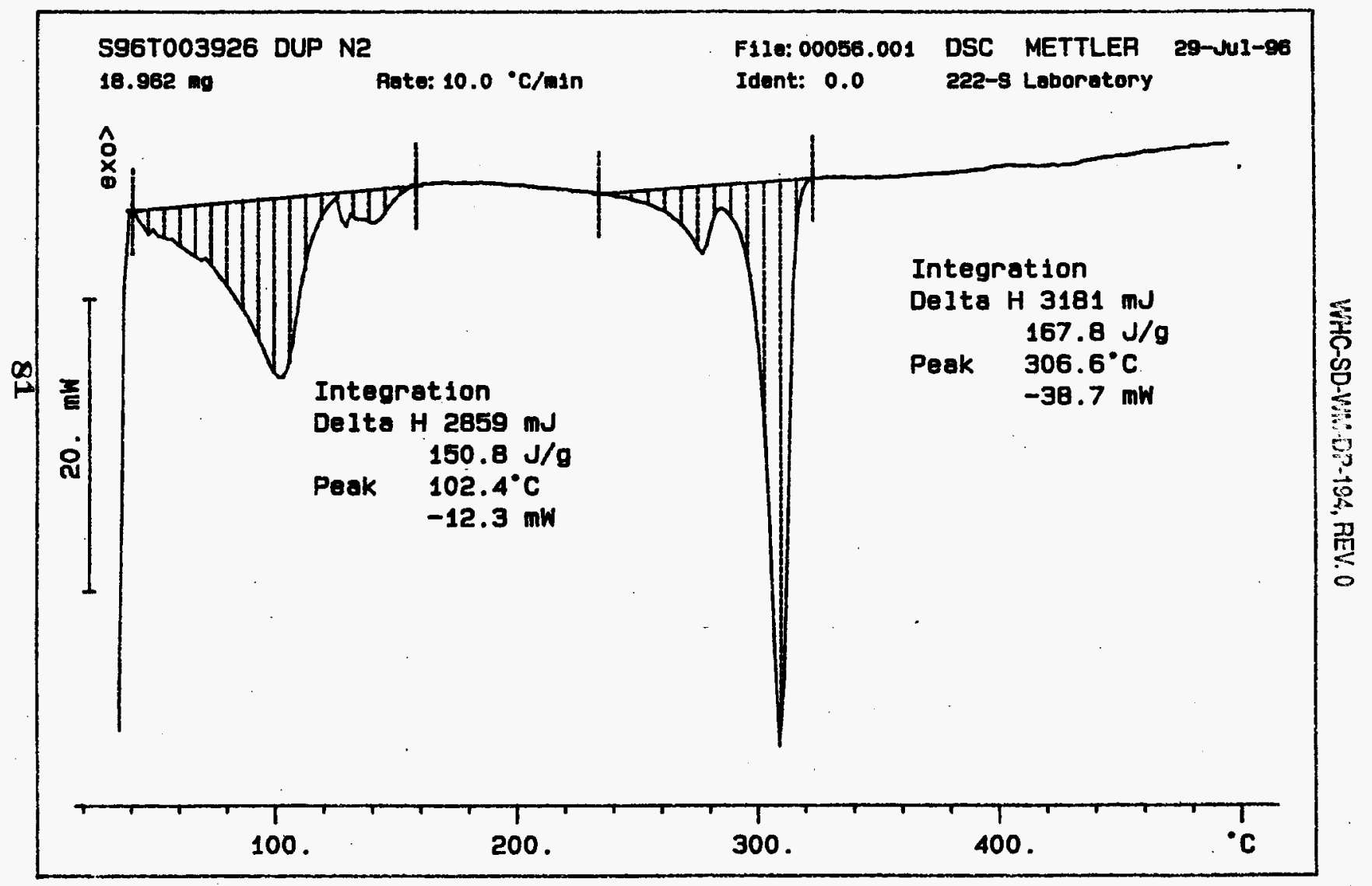




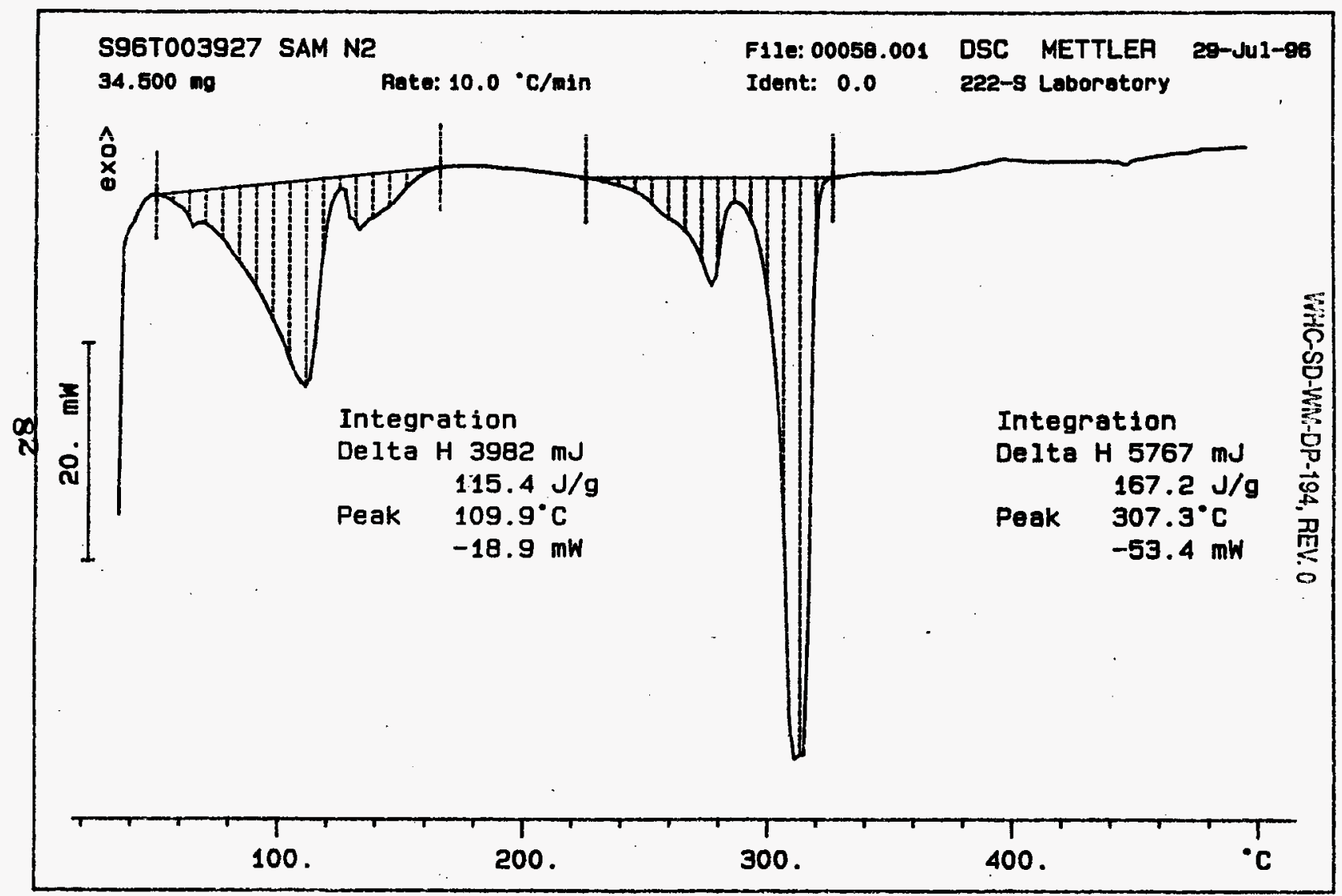




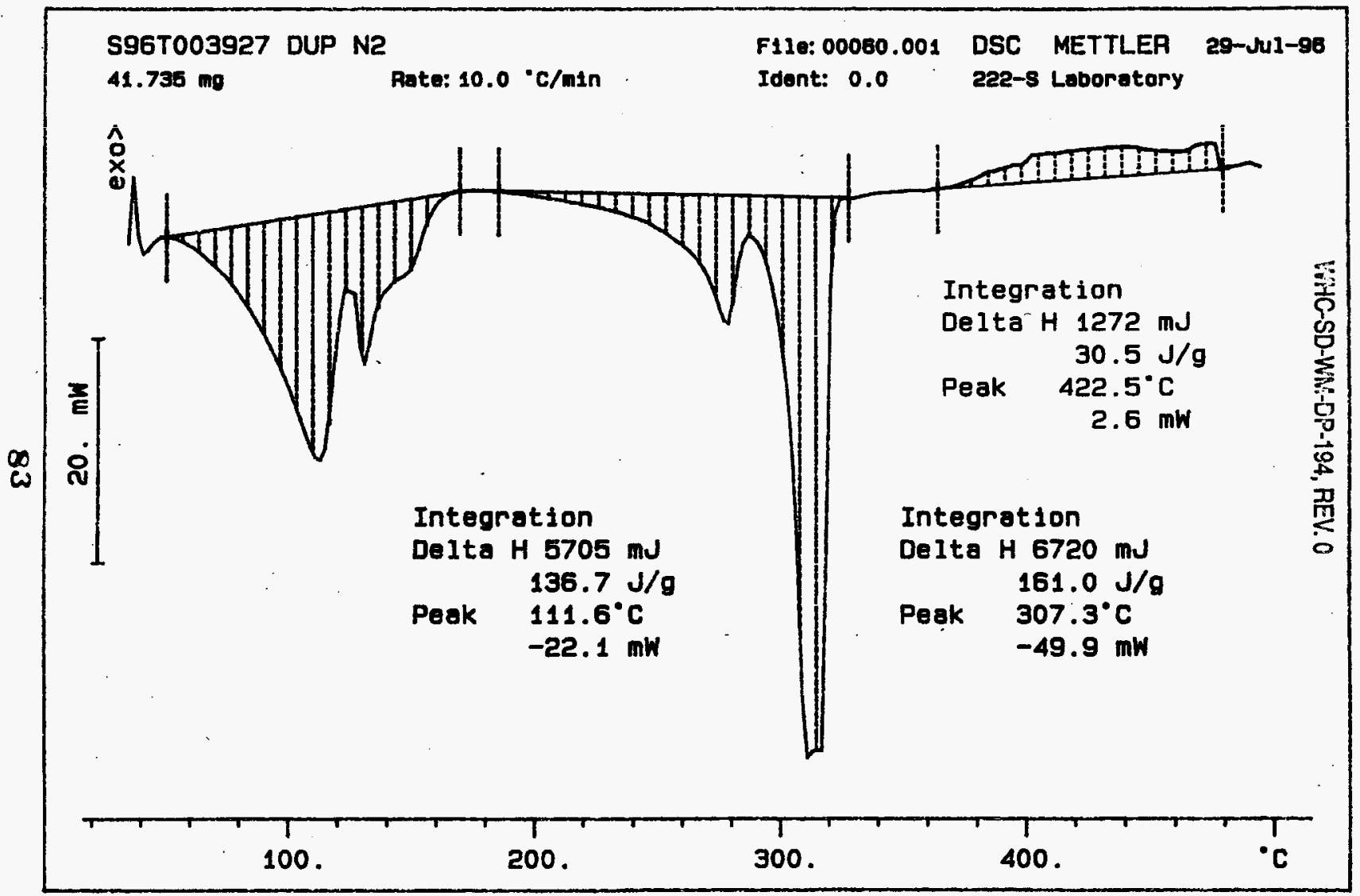




\section{LABCORE Data Entry Template for Worklist\#}

Analyst: KRM Instrument: DSCO I Book \#12N/4B

Method: LA-514-113 Rev/Mod C-1

Worklist Comment: S-109 DSC, RUN UNDER N2. RCJ

\begin{tabular}{|c|c|c|c|c|c|c|c|c|c|c|}
\hline \multirow[t]{2}{*}{ GROUP } & \multirow[t]{2}{*}{ PROJECT } & \multirow{2}{*}{$\begin{array}{l}S \text { TYPE } \\
1 \text { STD }\end{array}$} & \multirow[t]{2}{*}{ SAMPLE\# } & \multicolumn{2}{|c|}{ R A $\cdots . . . . .$. TEST $\cdots . . .}$. & \multirow{2}{*}{$\begin{array}{l}\text { MATRIX } \\
\text { SOLID }\end{array}$} & \multirow{2}{*}{$\begin{array}{l}\text { ACTUAL } \\
28.45 \\
\end{array}$} & \multirow{2}{*}{$\begin{array}{l}28.2 \\
28.2 \\
\end{array}$} & \multirow{2}{*}{$\begin{array}{l}\text { DL } \\
\quad N / A \\
\end{array}$} & \multirow{2}{*}{$\begin{array}{l}\text { UNIT } \\
\text { Joul es/g }\end{array}$} \\
\hline & & & & & osc-01 & & & & & \\
\hline 96000979 & s.109 & 2 SAMPLE & s96T004017 & 0 & DSC-01 & SOLID & $M / A$ & 31.7 & & Joules/ \\
\hline 96000979 & 5.109 & 3 IDUP & 5961004017 & 0 & DSC-01 & sotid & 31.7 & 47.8 & $N / A$ & Joules \\
\hline
\end{tabular}

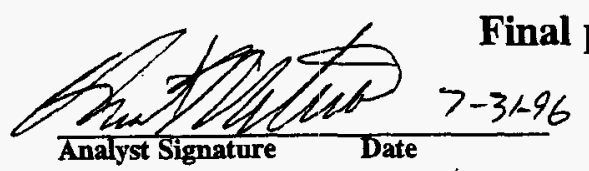

Analyst Signature

Venfied by

\section{Final page for worklist \#}

11309

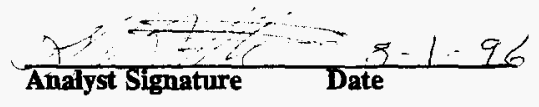

Data Entry Comments:

Units shown for QC (SPK \& STD) may not reflect the actual units. $D L=$ Detection Limit, $S=$ Worklist Slot Number, $R=$ Replicate Number, $A=$ Aliquot Code. 
SIGNATURE BELOW REPRESENTS CHEMICAL TECHNOLOGIST/CHEMIST THAT COMPLETED/VERIFIED THE CALIBRATION/ANALYSIS ON PAGES 85 TO 87.

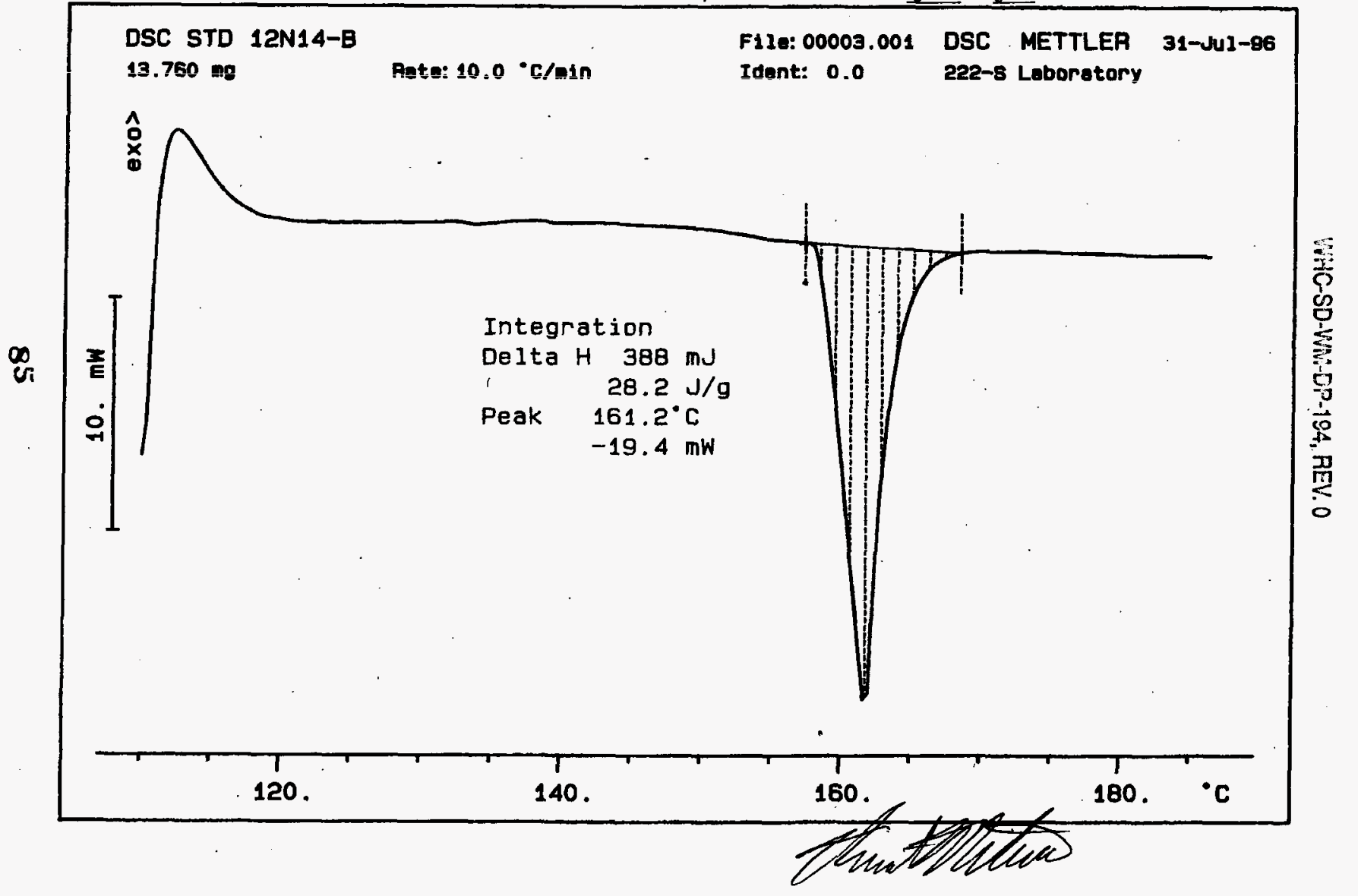




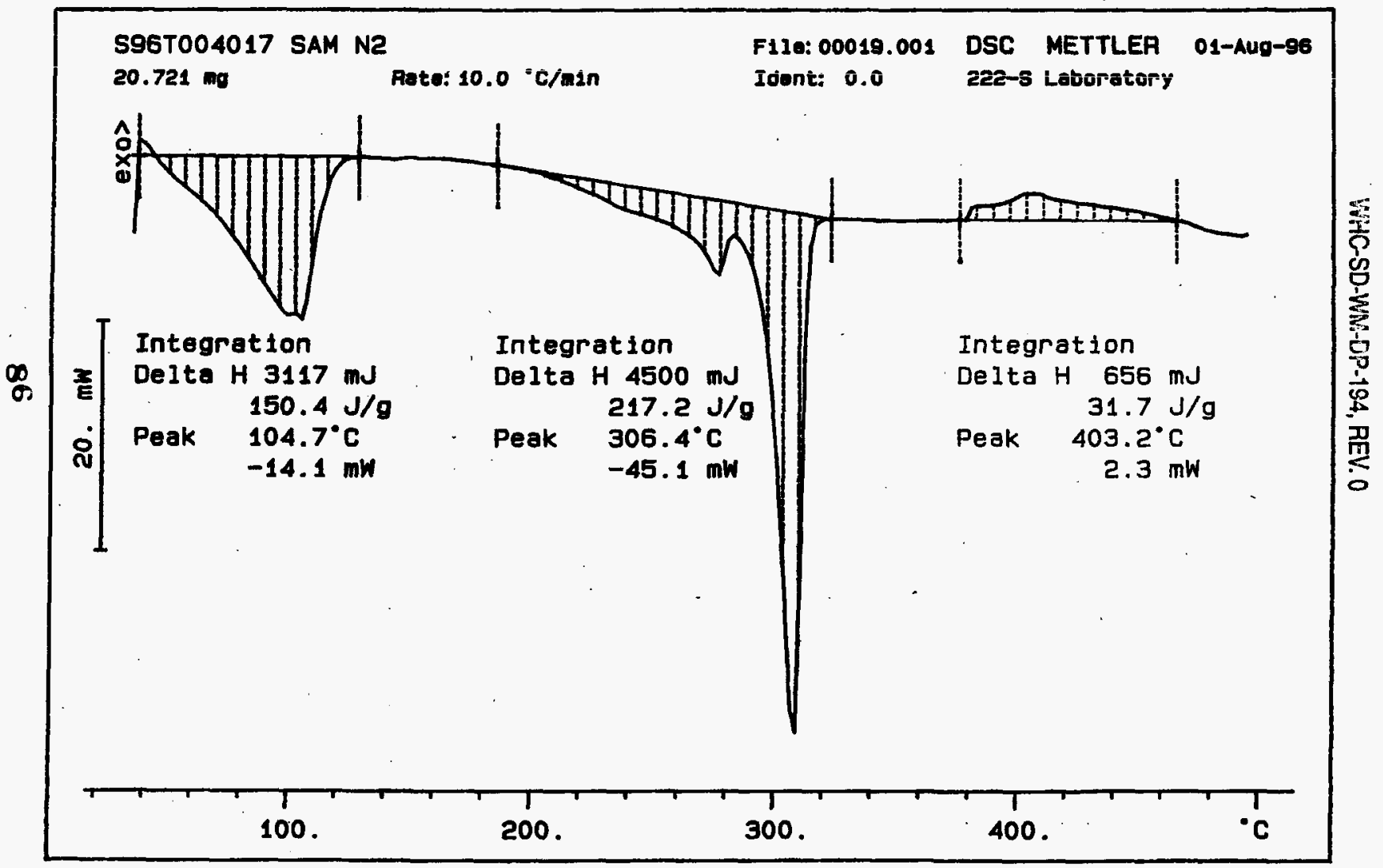




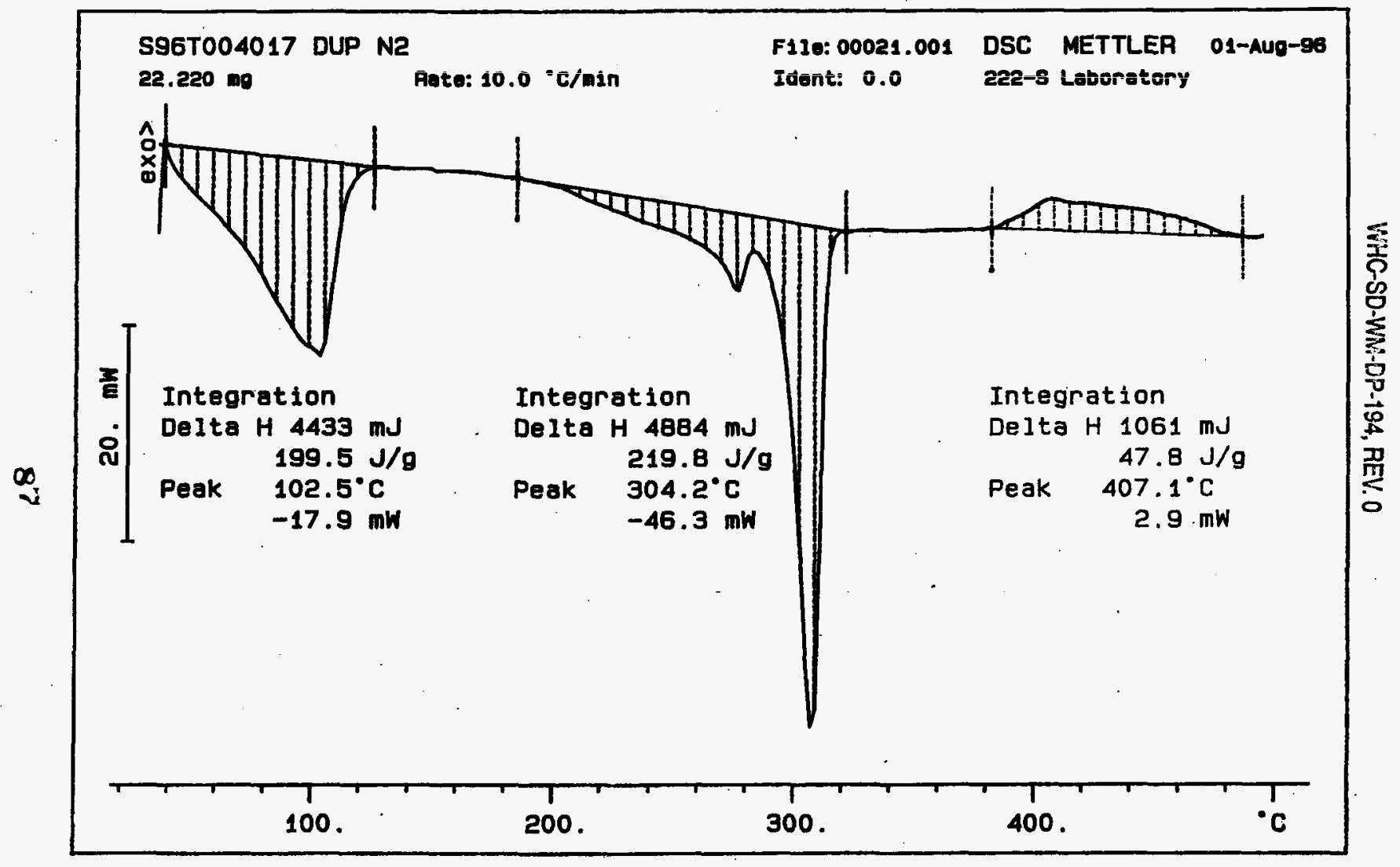


Analyst: $\quad$ BOY Instrument: $\mathrm{DSC} 01$

Book \#

Method: LA-514-113 Rev/Mod

Worklist Comment: Dry DSC for S-109. bdv

\begin{tabular}{|c|c|c|c|c|c|c|c|c|c|c|c|}
\hline GROUP & PROJECT & & TYPE & SAMPLE\# & RA & ….... TEST $\cdots$ & MATRIX & ACTUAL & FOUND & $D L$ & UNIT \\
\hline 96000979 & $s-109$ & 1 & SAMPLE & 5961003734 & 0 & DSC -02 & SOLID & $\mathrm{N} / \mathrm{A}$ & D & & Joules/g Dry \\
\hline 96000979 & s- 109 & 2 & DUP & 5961003734 & 0 & DSC -02 & SOL ID & Q & & $N / A$ & Joules/g Dry \\
\hline 96000961 & $s-109$ & 3 & SAMPLE & s96r 003800 & 0 & DSC-02 & SOL ID & $N / A$ & & & Joules/g Dry \\
\hline 96000961 & $s-109$ & 4 & DUP & $\$ 967003800$ & 0 & DSC-02 & SOLID & 4 & & $N / A$ & Joules/g Dry \\
\hline 96000961 & $s-109$ & 5 & SAMPLE & S96T003801 & 0 & $D S C-02$ & SOLID & $N / A$ & & & Joules/g Dry \\
\hline 96000961 & $s-109$ & 6 & DUP & \$96T003801 & 0 & DSC-02 & SOLID & & & $N / A$ & Joules/g Dry \\
\hline 96000961 & $s-109$ & 7 & SAMPLE & 5967003839 & 0 & DSC-02 & SOLID & $N / A$ & & & Joules/g Dry \\
\hline 96000961 & $s-109$ & 8 & DUP & 5967003839 & 0 & DSC-02 & SOLID & & & $N / A$ & Joules/9 Dry \\
\hline 96000979 & $s-109$ & 9 & SAMPLE & 5961003759 & 0 & DSC.02 & SOLID & $\mathrm{N} / \mathrm{A}$ & & & Joules/g Dry \\
\hline 96000979 & $s-109$ & 10 & DUP & S961003759 & 0 & DSC-02 & SOLID & & & $N / A$ & Joules/g Dry \\
\hline 96000979 & $s-109$ & 11 & SAMPLE & S961003760 & 0 & DSC-02 & SOLID & $N / A$ & & & Joules/g Dry \\
\hline 96000979 & $s-109$ & 12 & DUP & S96T003760 & 0 & DSC- 02 & SOLID & & 1.2 & $N / A$ & Joules/g Dry \\
\hline 96000979 & $s-109$ & 13 & SAMPLE & S96T003922 & 0 & DSC-02 & SOLID & $N / A$ & & & Joules/g Dry \\
\hline 96000979 & $s-109$ & 14 & DUP & S96T003922 & 0 & $05 C-02$ & SOLID & 0 & & N/A & Joules/g Dry \\
\hline
\end{tabular}

\section{Final page for worklist \# 11599}

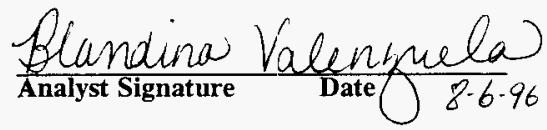

Analyst Signature Date

Data Entry Comments:

Units shown for $Q C$ (SPK \& STD) may not reflect the actual units. $D L=$ Detection Limit, $S=$ Worklist Slot Number, $R=$ Replicate Number, $A:=$ Aliquot Code. 
Analyst: $\mathrm{BOV}$ Instrument: $\mathrm{DSC} 01$

Book \#

Method: LA-514-113 Rev/Mod

Worklist Comment: Dry DSC for S-109. bdv

\begin{tabular}{|c|c|c|c|c|c|c|c|c|c|c|}
\hline GROUP & PROJECT & $S$ TYPE & SAMPLE\# & $R$ & --TEST--..... & MATRIX & ACTUAL & FOUND & $D L$ & UNIT \\
\hline 96000979 & s-109 & 1 SAMPLE & S96T003923 & 0 & DSC-02 & SOLID & $N / A$ & $\varnothing$ & & Joules/g Dry \\
\hline 96000979 & $s-109$ & 2 DUP & 596T003923 & 0 & DSC-02 & SOLID & $\varnothing$ & 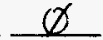 & N/A & Joules/g Dry \\
\hline 96000979 & s- 109 & 3 SAMPLE & S96T003924 & 0 & DSC-02 & SOLID & N/A & $\phi$ & & Joules/g Dry \\
\hline 96000979 & $s-109$ & 4 DUP & 596T003924 & 0 & DSC-02 & SOLID & $\varnothing$ & $\phi$ & $N / A$ & Joules/g Dry \\
\hline 96000979 & s- 109 & 5 SAMPLE & S96T003925 & 0 & DSC-02 & SOLID & N/A & & & Joules/g Dry \\
\hline 96000979 & s- 109 & 6 DUP & 596T003925 & 0 & DSC- 02 & SOLID & & & N/A & Joules/g Dry \\
\hline 96000979 & s-109 & 7 SAMPLE & S96T003926 & 0 & DSC-02 & SOLID & $N / A$ & 3 & & Joules/g Dry \\
\hline 96000979 & S-109 & 8 DUP & S96T003926 & 0 & DSC- 02 & SOLID & 1.3 & $\varnothing$ & $N / A$ & Joules/g Dry \\
\hline 96000979 & s- 109 & 9 SAMPLE & S96T003927 & 0 & DSC- 02 & SOLID & N/A & $\not$ & & Joules/g Dry \\
\hline 96000979 & s- 109 & 10 DUP & S96T003927 & 0 & DSC-02 & SOLID & 1 & 3 & N/A & Joules/g Dry \\
\hline 96000979 & s. 109 & 11 SAMPLE & \$96T004017 & 0 & DSC- 02 & SOLID & $N / A$ & 4.3 & & Joules/g Dry \\
\hline 96000979 & s. 109 & 12 DUP & S96T004017 & 0 & DSC-02 & SOLID & 34 & 51.7 & N/A & Joules/g Dry \\
\hline
\end{tabular}

Final page for worklist \# 11600

Blandina Vatenumela) Analyst Signature Date) $8.6-96$

\author{
Analyst Signature Date
}

Data Entry Comments:

Units shown for QC (SPK \& STD) may not reflect the actual units. $D L=$ Detection Limit, $S=$ Worklist Slot Number, $R=$ Replicate Number, $A=$ Aliquot Code . 


\section{LABCORE Data Entry Template for Worklist\#}

Analyst: $\quad$ EAL Instrument: DSCO I Book\# 12 N $14 B$

Method: LA-514-113 Rev/Mod C-I

Worklist Comment: S-109 FOR DSC PLEASE RUN UNDER N2

\begin{tabular}{|c|c|c|c|c|c|c|c|c|c|c|}
\hline \multirow[t]{2}{*}{ GROUP } & \multirow[t]{2}{*}{ PROJECT } & $S$ TYPE & SAMPLE* & \multicolumn{2}{|c|}{$R \wedge \cdots \ldots . . . .$. TEST $\cdots . .}$. & MATRIX & ACTUAL & FOUND & $\cdot D L$ & UNIT \\
\hline & & 1 STD & & & DSC-01 & LIQUID & 28 & 26. & N/A & Joules $/ 9$ \\
\hline 96000961 & s-109 & 2 SAMPLE & 5967004023 & 0 & DSC-01 & HOUID & $\mathbf{N} / \mathbf{A}$ & $\varnothing$ & & Joules/g \\
\hline 96000961 & s-109 & 3 DUP & 5967004023 & 0 & DSC-01 & LIOUID & & & N/A & Joules/g \\
\hline 96000961 & $s-109$ & 4 SAMPLE & \$96T004033 & 0 & DSC-01 & LIOUID & 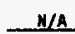 & & & Joules/g \\
\hline 96000961 & s. 109 & 5 DUP & S96T004033 & 0 & DSC-01 & LIQUID & & & $\mathrm{N} /$ & Joules/g \\
\hline
\end{tabular}

\section{Final page for worklist \#}

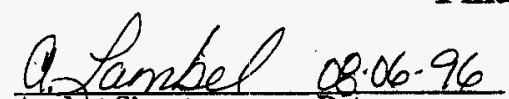

Analyst Signature Date

Verified/Validated by

Blandina

Valempela 8.8 .96

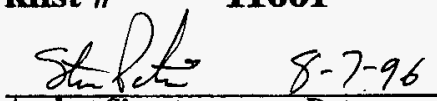

Analyst Signature Date

Data Entry Comments:

Units shown for QC (SPK \& STD) may not reflect the actual units. $D L=$ Detection Limit, $S=$ Worklist Slot Number, $R=$ Replicate Nimber, $A=$ Aliquot Code. 


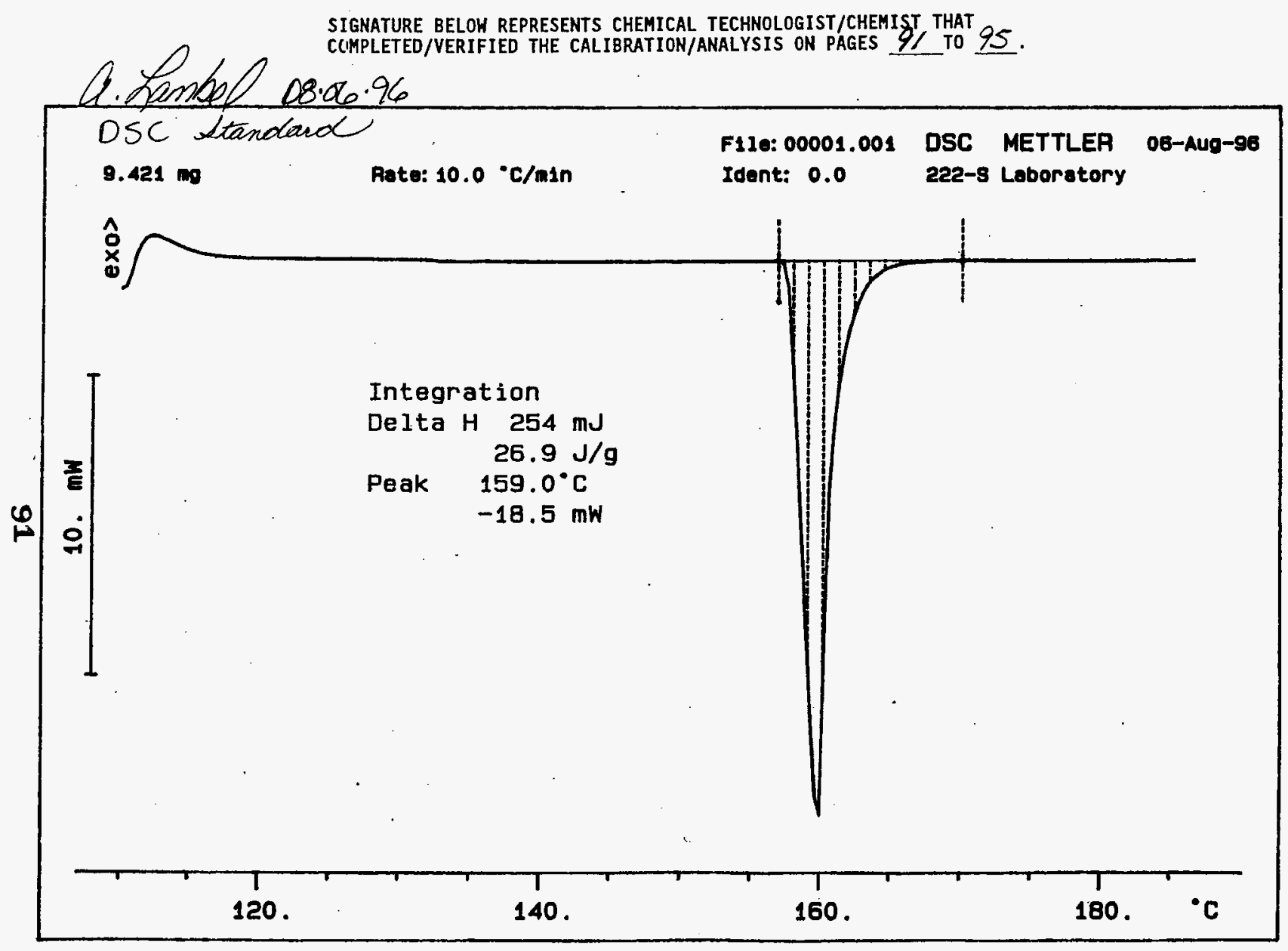


WHC-SD-WM-EP-194, REV. 0

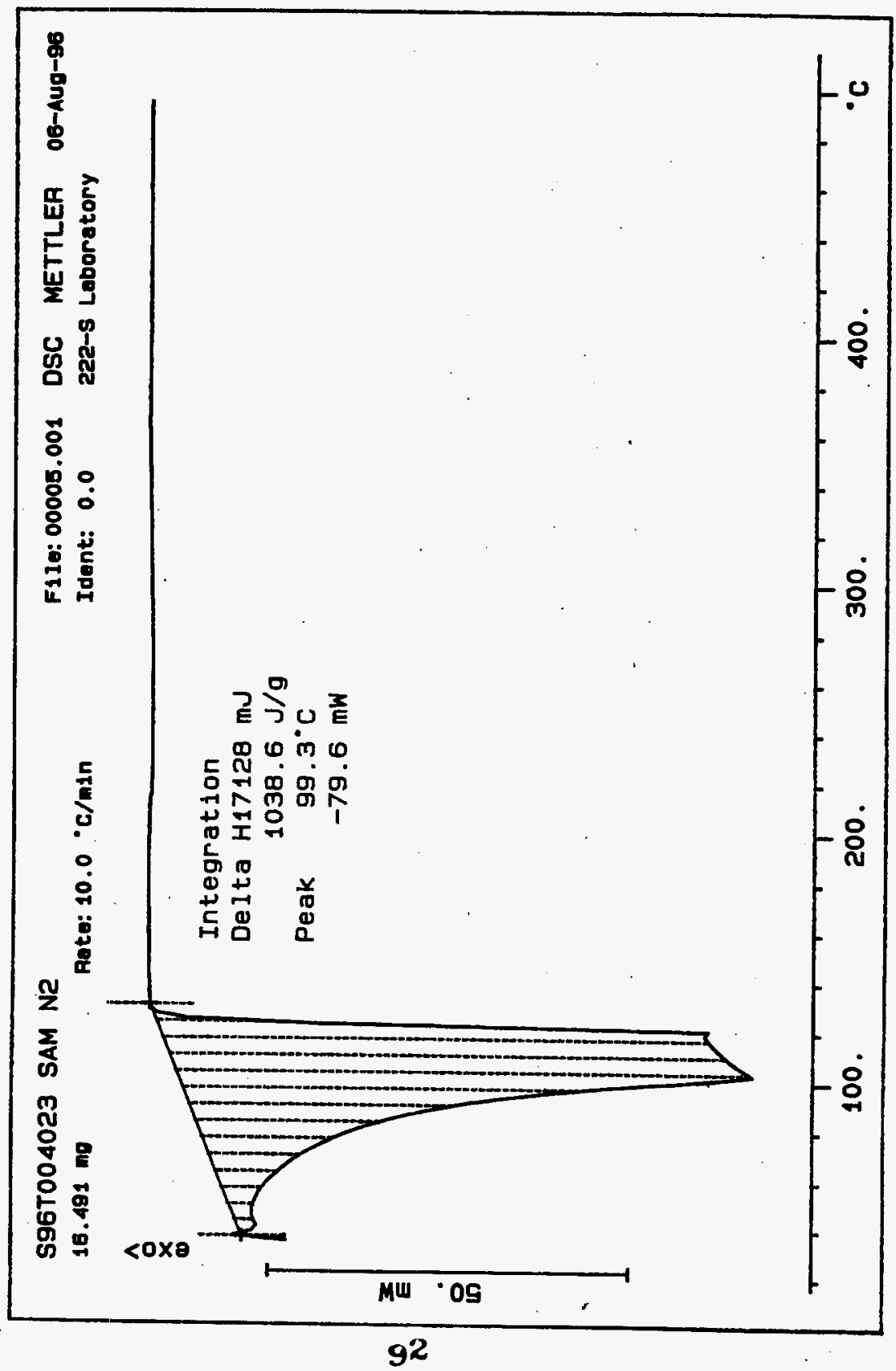




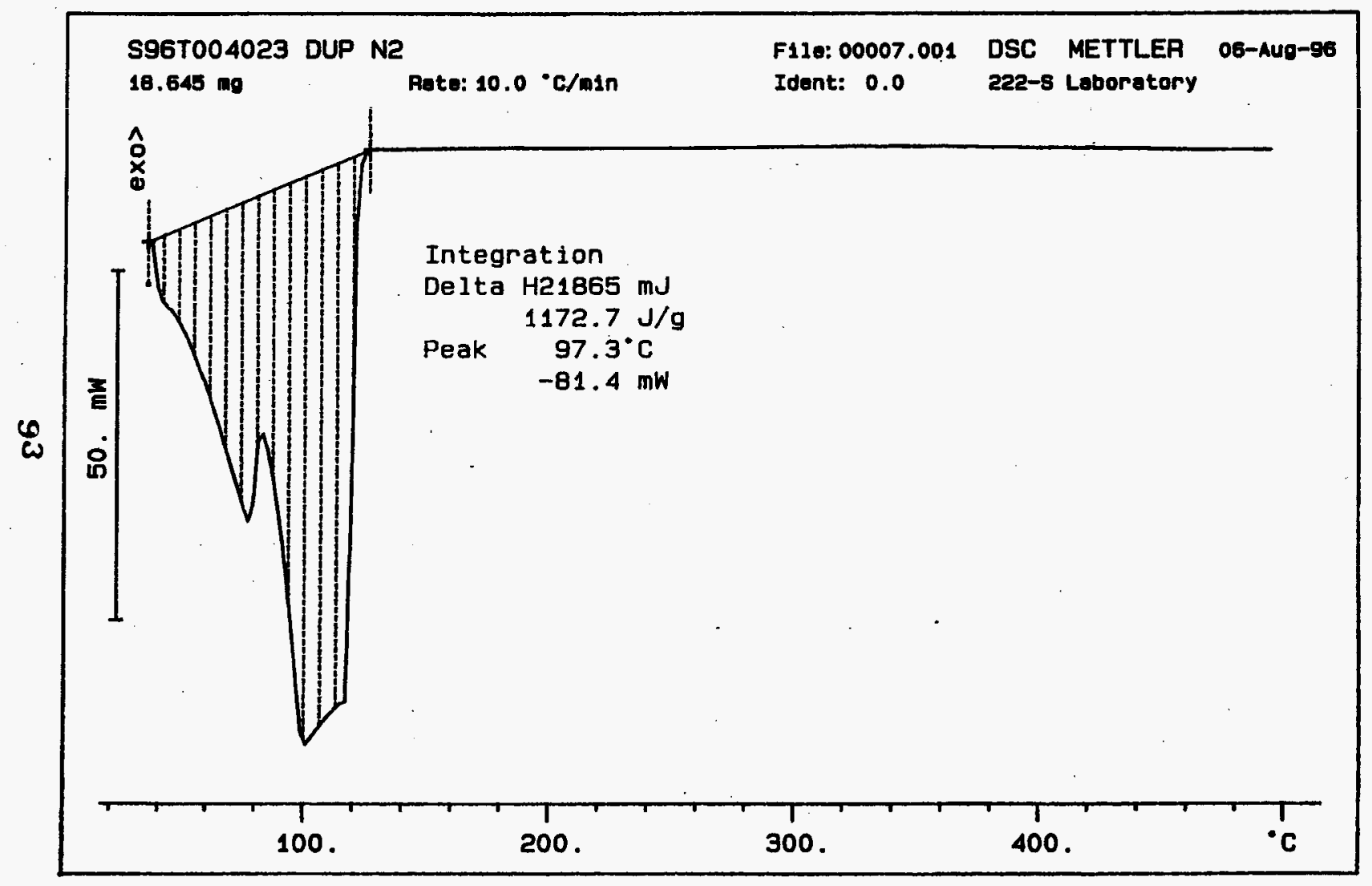




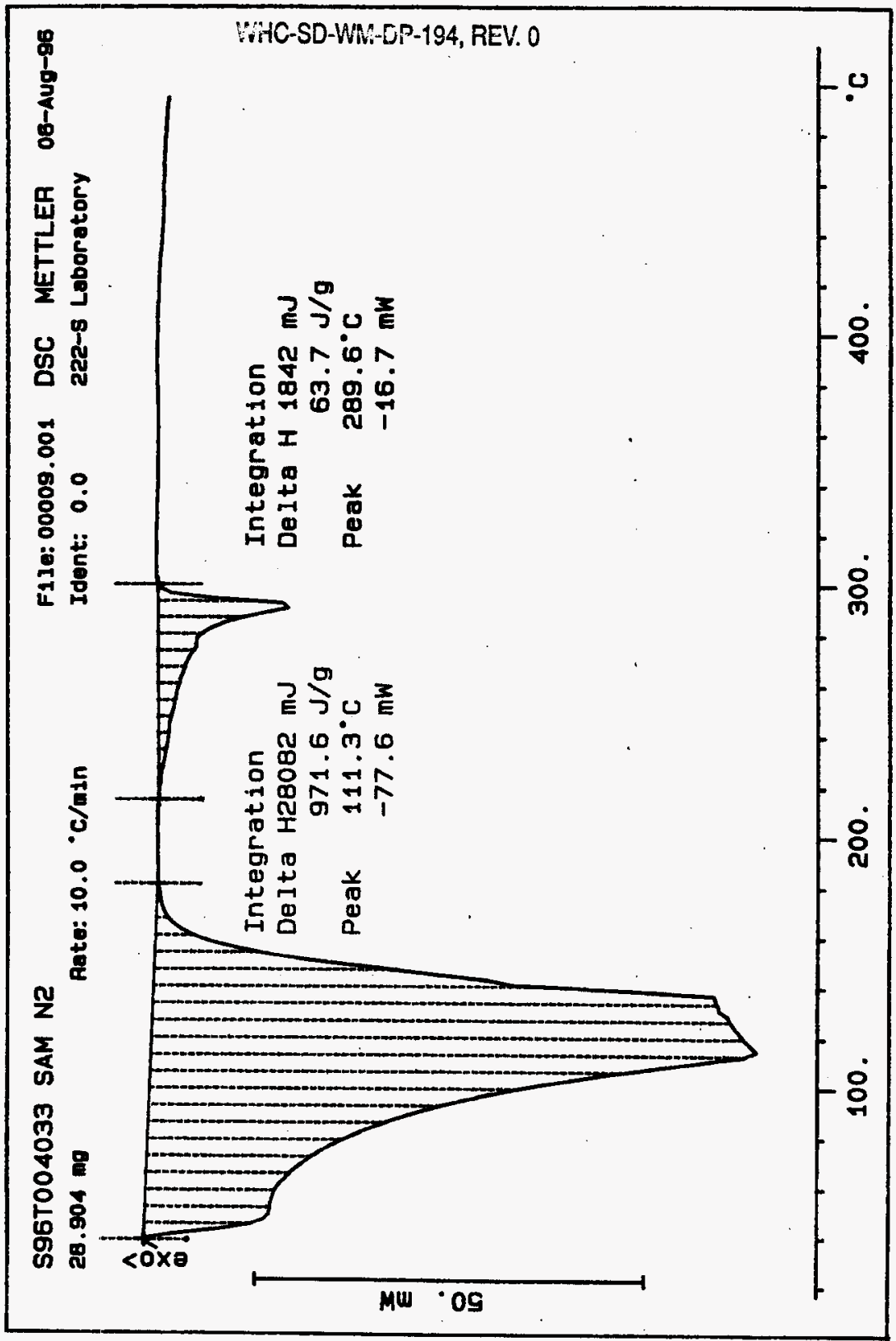

94 


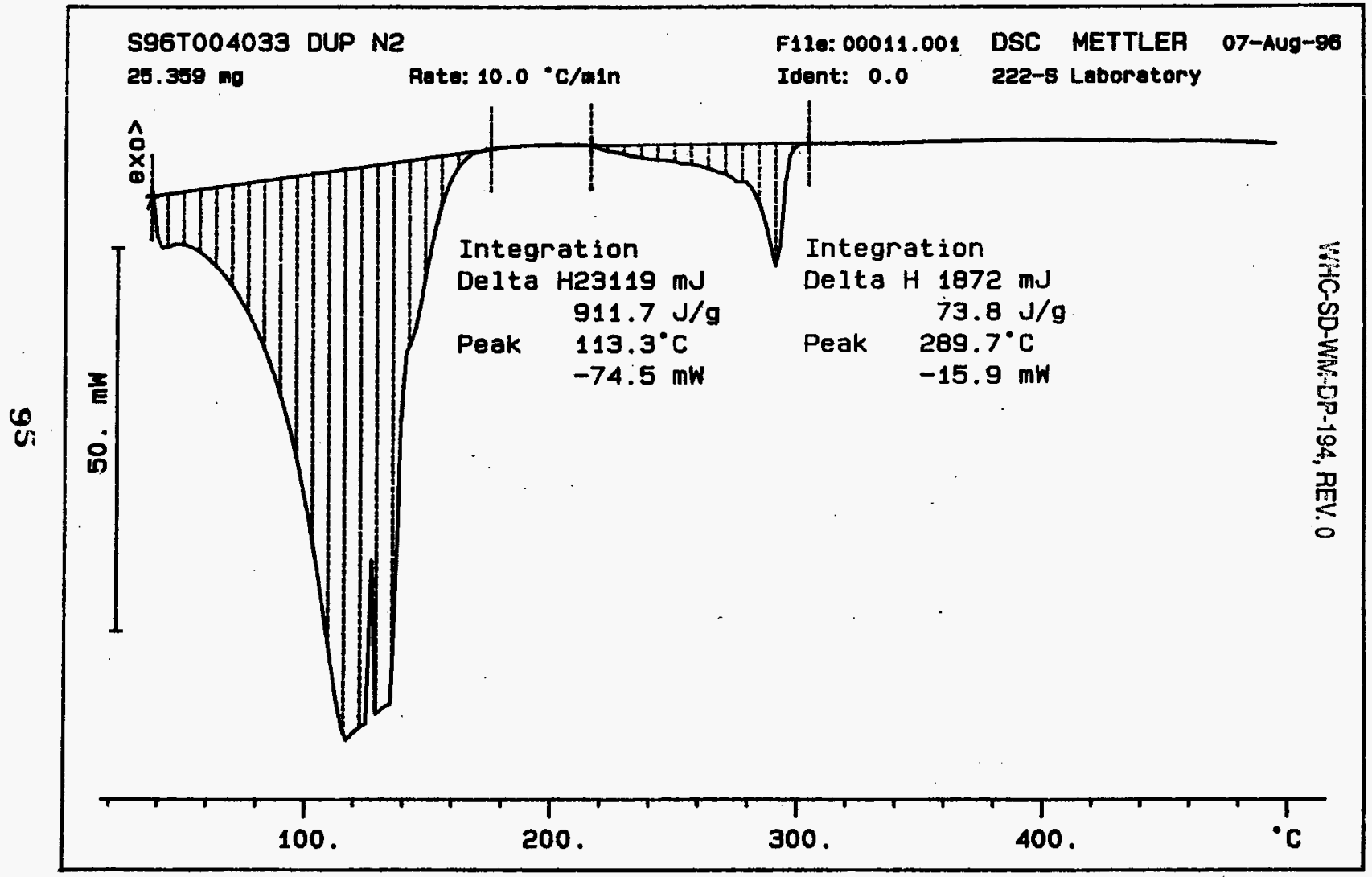




\begin{tabular}{|c|c|c|c|c|c|}
\hline Analyst: & $\frac{E A L}{14-113 \mathrm{Re}}$ & $\begin{array}{l}\text { Instrument: } \\
\mathrm{C}-1\end{array}$ & DSCO & 1 & Book $\# \frac{\frac{t 2 N 44 A}{8-8-96}}{\text { Bor }} 12 N 14 B$ \\
\hline
\end{tabular}

Worklist Comment: S-109 FOR DSC PLEASE RUN UNDER N2

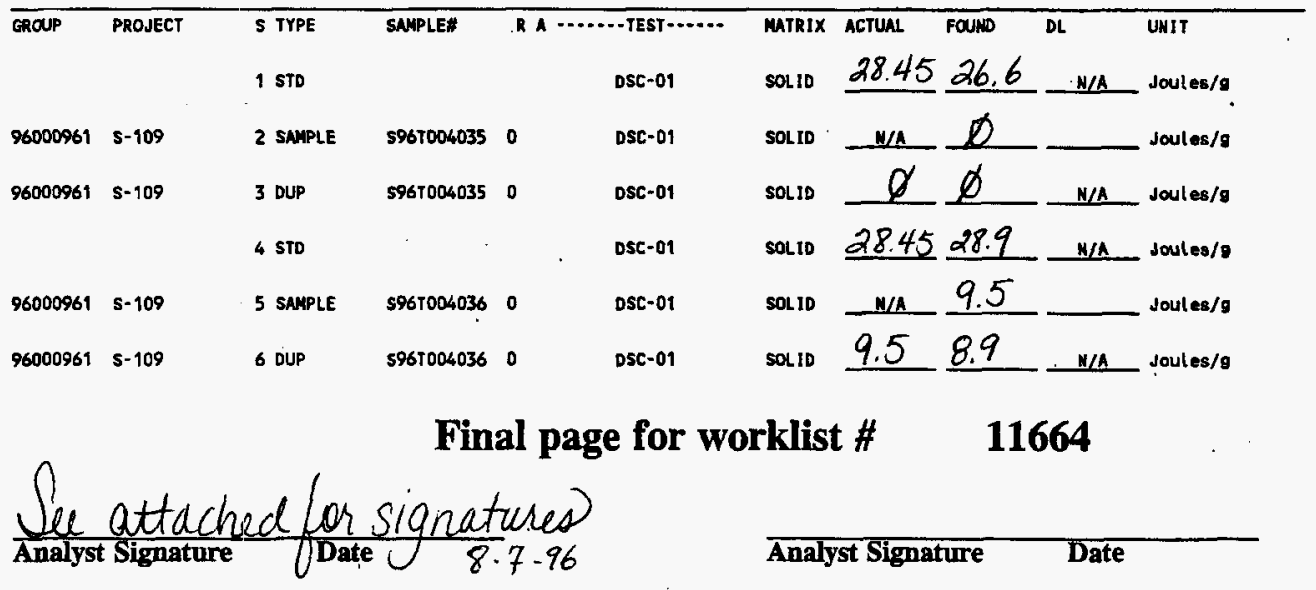

Data Entry Comments:

Units shown for QC (SPK \& STD) may not reflect the actual units. $D L=$ Detection Limit, $S=$ Worklist Slot Number, $R=$ Replicate Number, $A=$ Aliquot Code. 


\section{LABCORE Data Entry Template for Worklist\#}

Analyst:

Instrument: DSCO

Book \#

Method: LA-514-113 Rev/Mod

Worklist Comment: S-109 FOR DSC PLEASE RUN UNDER N2

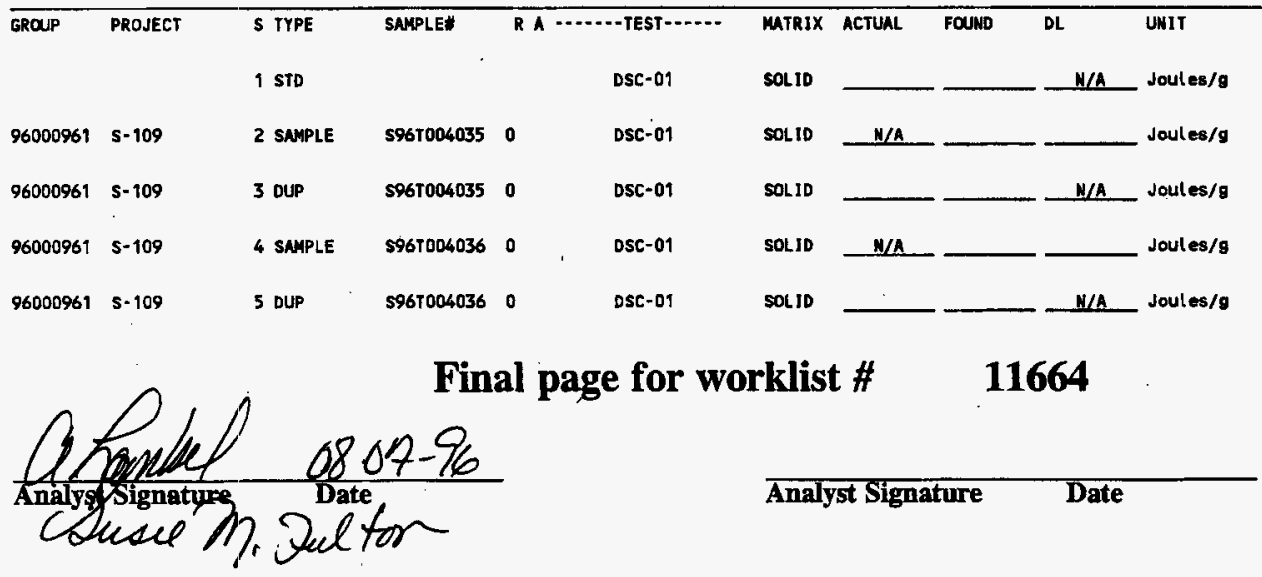

Data Entry Comments:

Units shown for $Q C$ (SPK \& STD) may not reflect the actual units. $D L=$ Detection Limit, $S=$ Worklist Slot Number, $R=$ Replicate Number $A=$ Aliquot Code. 


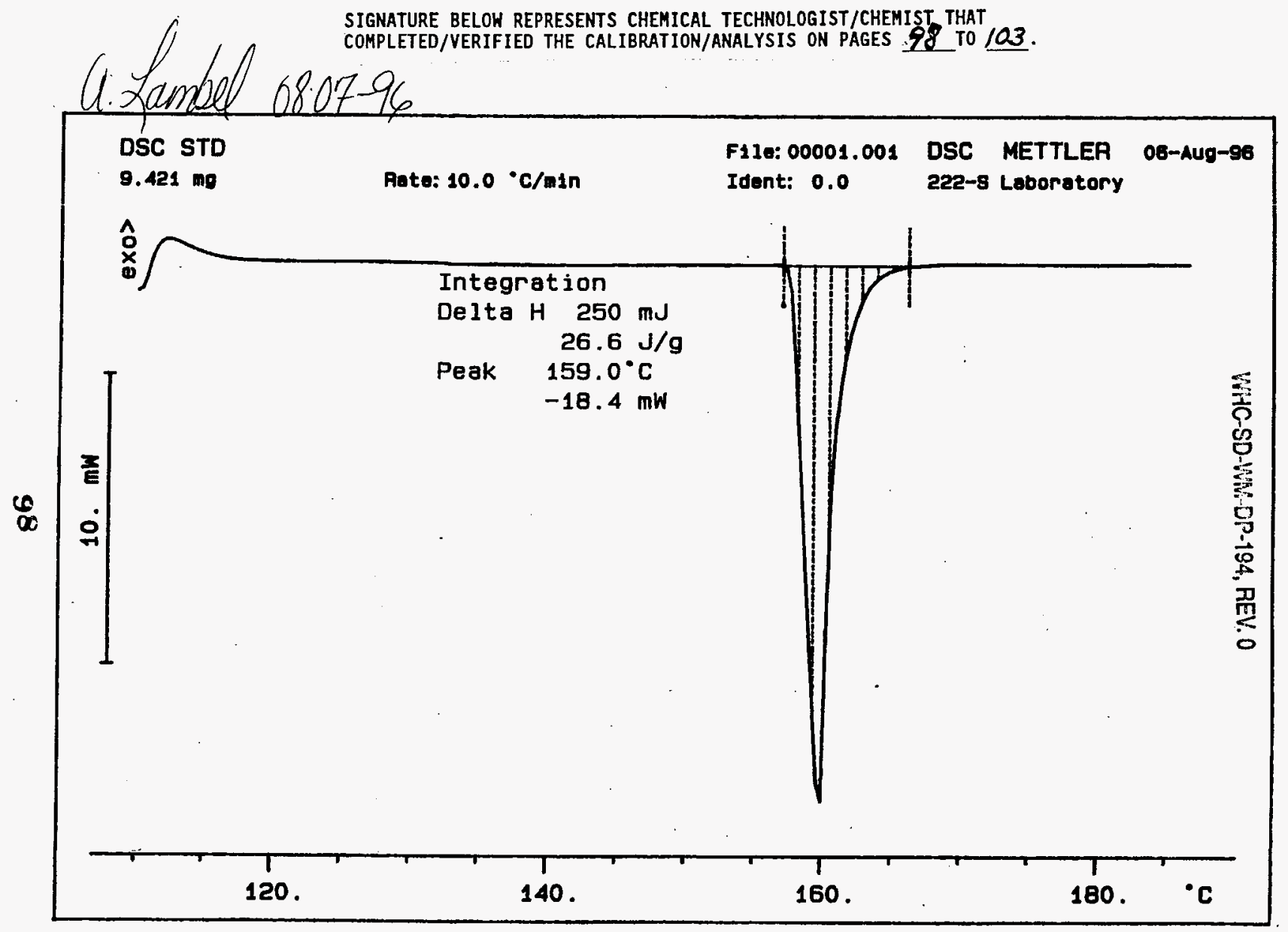




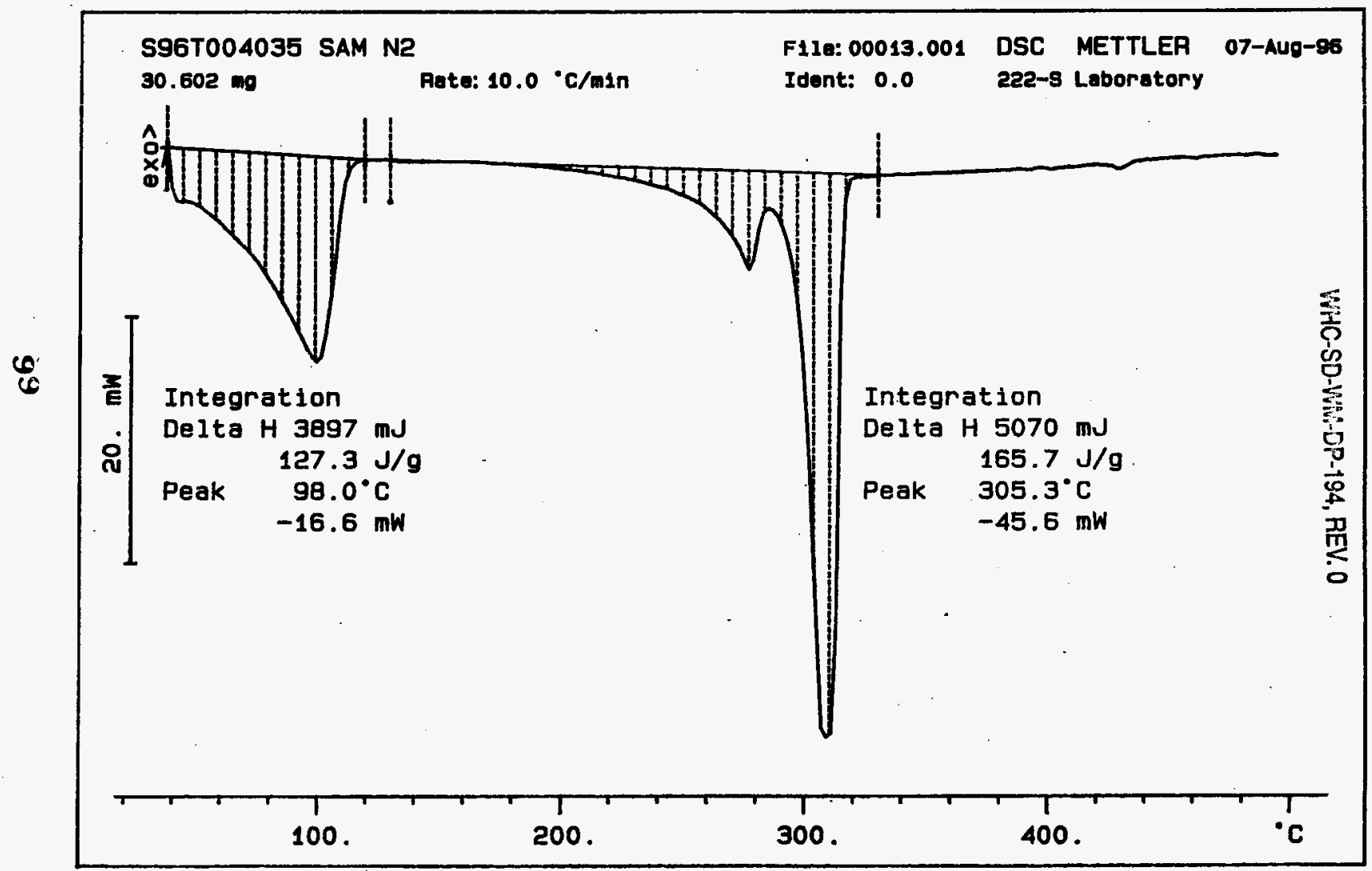




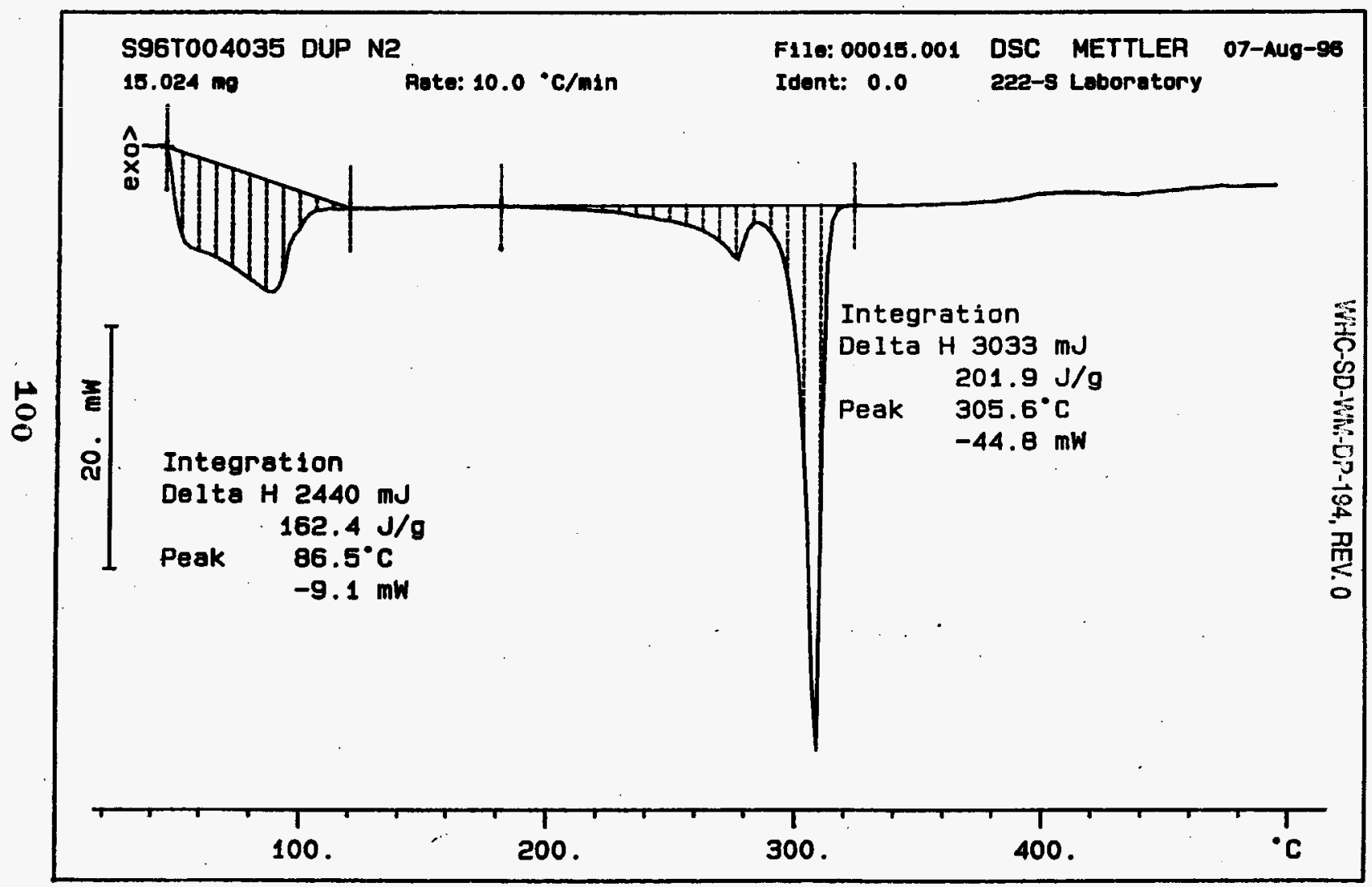




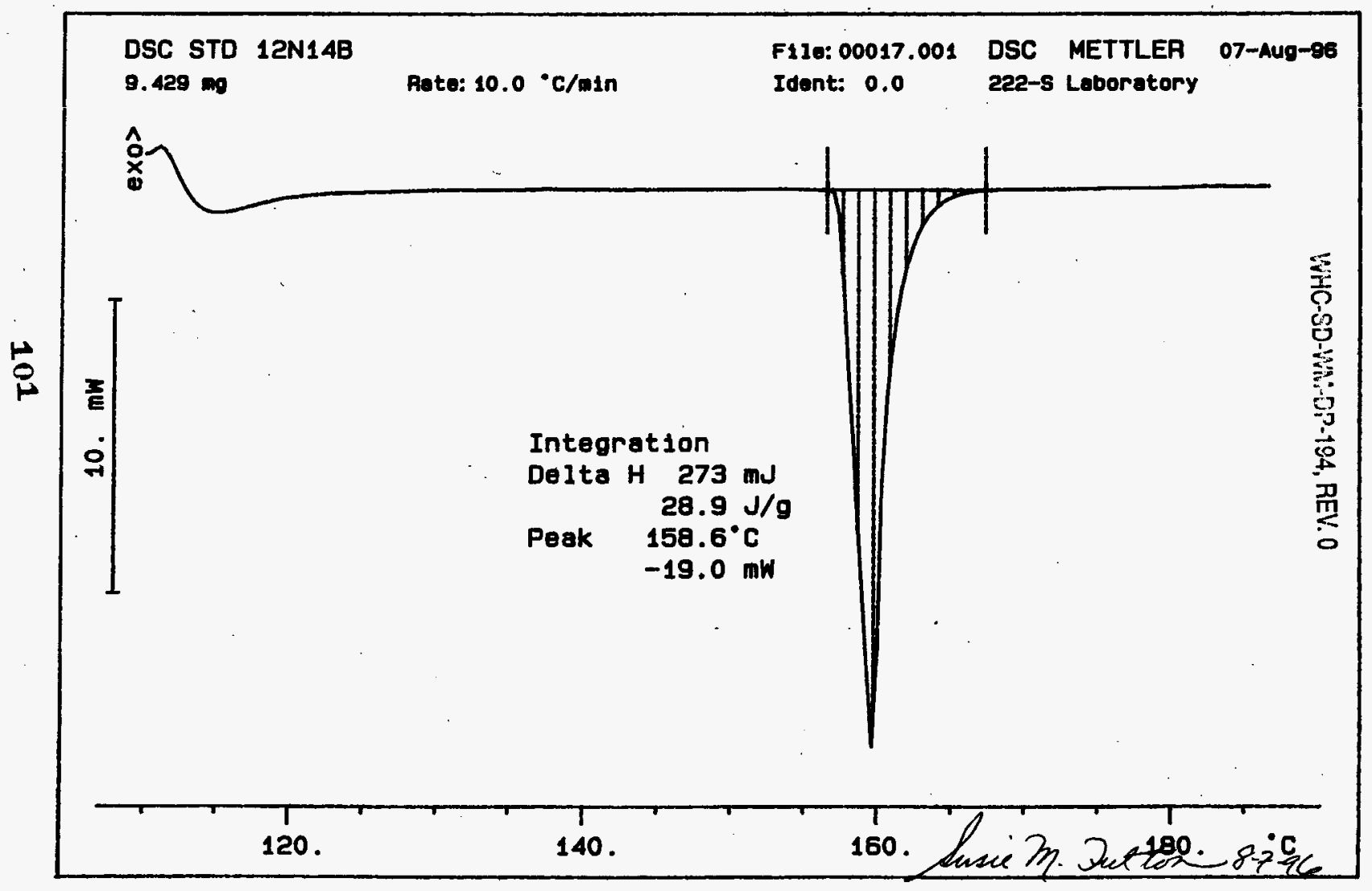




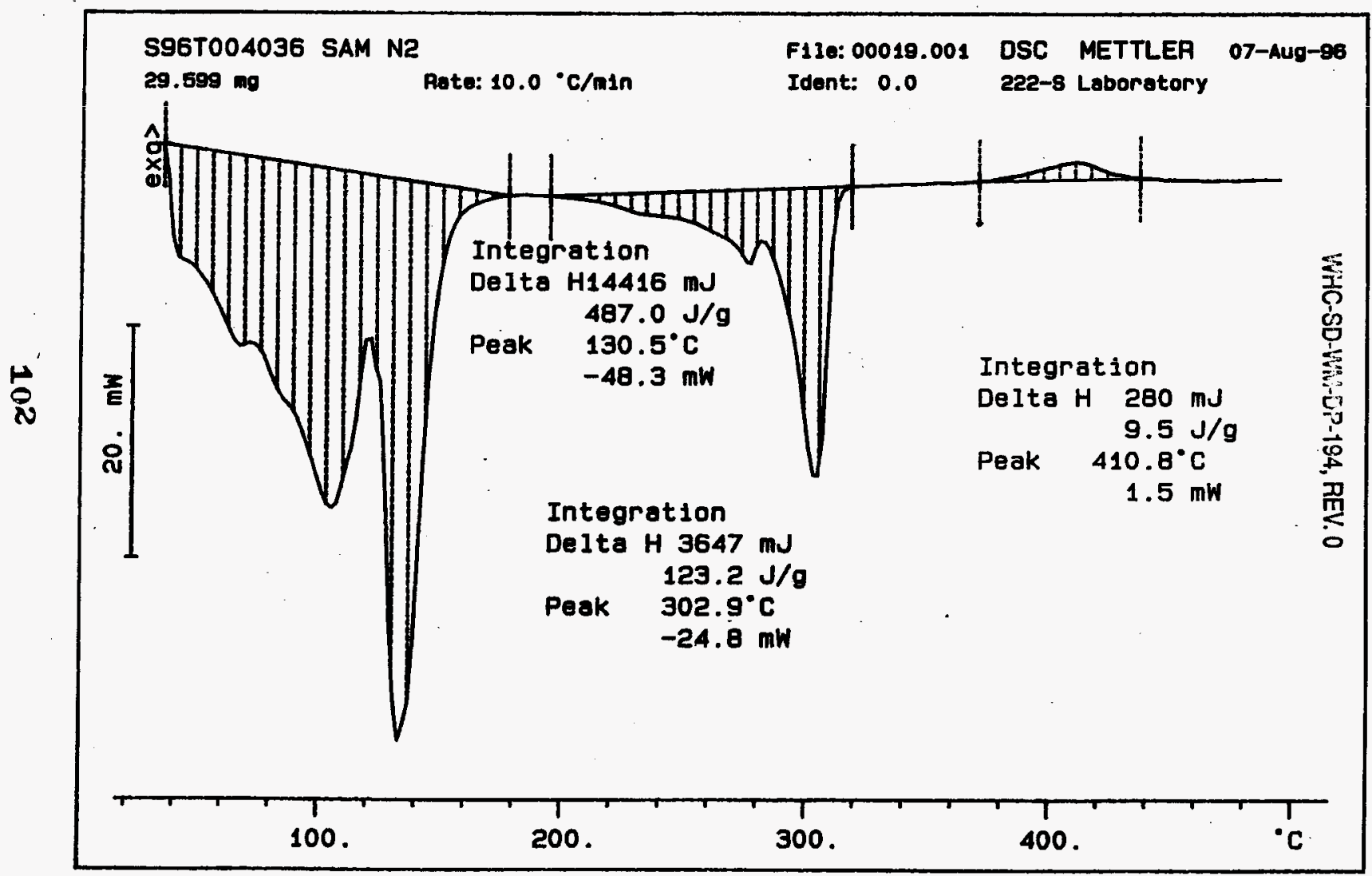




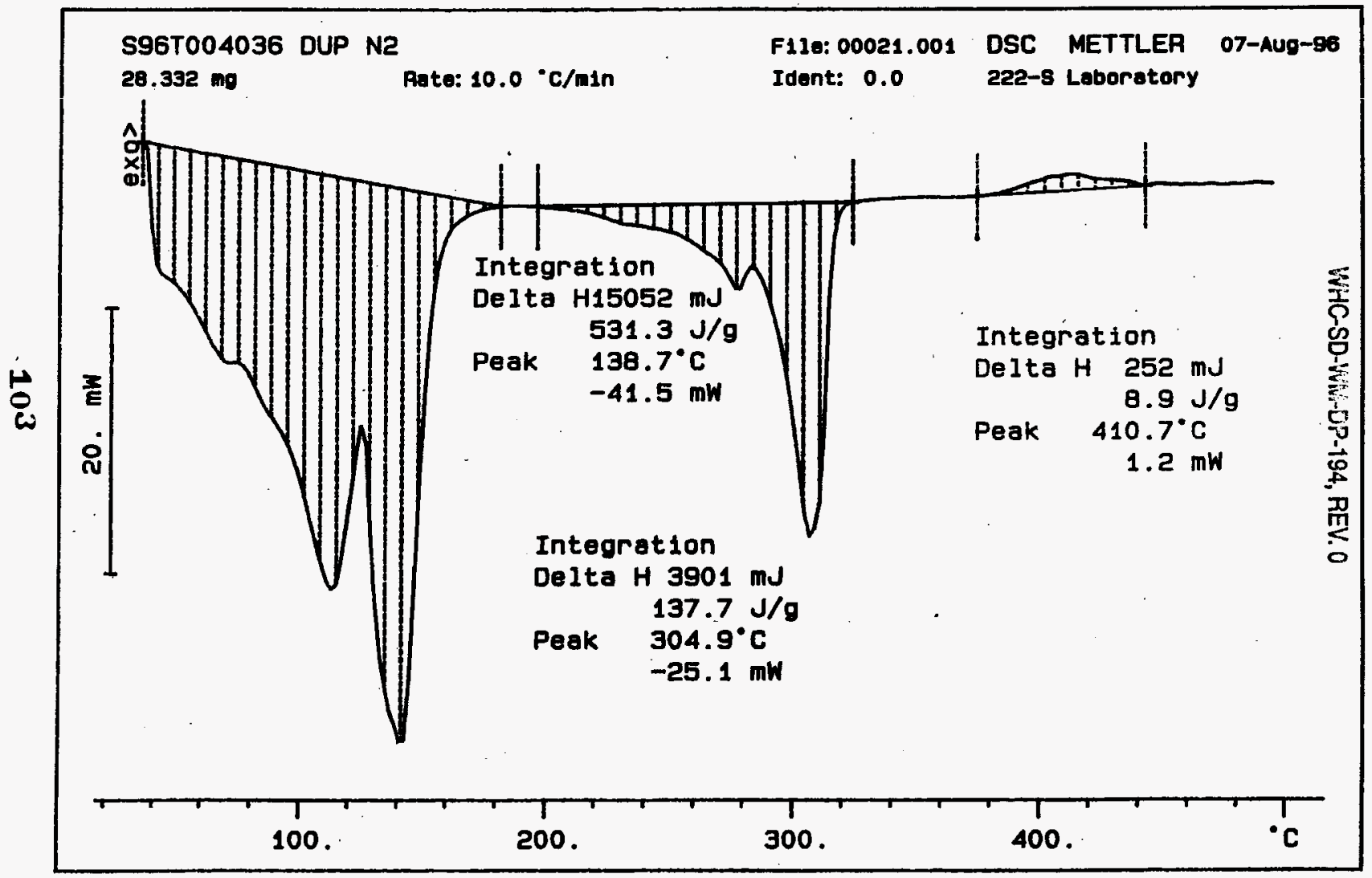


WHC-SD-WA-OP-104, REV. 0

worklistrpt Version $2.105 / 15 / 95$

08/07/96 08:46

\section{LABCORE Data Entry Template for Worklist\#}

Analyst: $\quad B D Y$

Instrument: DSC01

Book \#

Method: LA-514-113 Rev/Mod

Worklist Comment: Dry DSC for S-109. bdv

\begin{tabular}{|c|c|c|c|c|c|c|c|c|c|c|}
\hline GROUP & PROJECT & S TYPE & SAMPLE\# & R A & $\ldots \ldots$ TEST $\ldots \ldots$ & MATRIX & ACTUAL & FOUND & $\mathrm{DL}$ & UNIT \\
\hline 96000961 & s- 109 & 1 SAMPLE & 5967004035 & 0 & DSC-02 & SOLID & N/A & & & Joules/g Dry \\
\hline 96000961 & s- 109 & 2 DUP & 5967004035 & 0 & DSC-02 & SOL1D & $D$ & & $N / A$ & Joules/g Dry \\
\hline 96000961 & $s-109$ & 3 SAMPLE & $\$ 96 T 004036$ & 0 & DSC-02 & SOLID & N/A & & & Joules/g Dry \\
\hline 96000961 & s-109 & 4 DUP & S96T004036 & 0 & DSC-02 & SOLID & & & $N / A$ & Joules/g DrY \\
\hline 96000961 & $s-109$ & 5 SAMPLE & S96T004023 & 0 & DSC-02 & LIOUID & $N / A$ & & & Joules/g Dry \\
\hline 96000961 & $s-109$ & 6 DUP & S96T004023 & 0 & $\mathrm{DSC}-02$ & LIQUID & 60 & & N/A & Joules/g Dry \\
\hline 96000961 & $s-109$ & 7 SAMPLE & $\$ 961004033$ & 0 & DSC-02 & LIQUID & $N / A$ & & & Joules/g Dry \\
\hline 96000961 & $s-109$ & 8 DUP & $596 T 004033$ & 0 & DSC-02 & LIOUID & & & $\mathrm{N} / \mathrm{A}$ & Joules/g Dry \\
\hline
\end{tabular}

\section{Final page for worklist \# 11699}

\section{Blandina Valenzuela \\ Analyst Signature Qate 8.8 .96}

Analyst Signature Date

Data Entry Comments:

Units shown for $Q C$ (SPK \& STD) may not reflect the actual units, $D L=$ Detection Limit, $S=$ Worklist Slot Number,

$\boldsymbol{R}=$ Replicate Number, $A=$ Aliquot Code. 
Analyst: KRM Instrument: TGA03 Book \# $12 \mathrm{~N} 8 \mathrm{~A}$

Method: LA-514-114 Rev/Mod C-1

Worklist Comment: S-109 TGA, RUN UNDER N2. RCJ

\begin{tabular}{|c|c|c|c|c|c|c|c|c|c|c|}
\hline GROP & PROJECT & $\begin{array}{l}\text { S TYPE } \\
1 \text { STD }\end{array}$ & SAMPLE* & $R A$ & TGA-03 & $\begin{array}{l}\text { MATRIX } \\
\text { SOLID }\end{array}$ & $\begin{array}{l}\text { ACTUAL } \\
59.2 \\
\end{array}$ & $\begin{array}{l}\text { FOUND } \\
59.2 *\end{array}$ & $\begin{array}{l}\text { DL } \\
K_{\text {W/A }}\end{array}$ & $\begin{array}{l}\text { UnIT } \\
x\end{array}$ \\
\hline 96000979 & S- 109 & 2 SAMPLE & 5967003734 & 0 & TGA-03 & 50210 & $N / A$ & $1.0^{\circ}$ & & $x$ \\
\hline 96000979 & s-109 & 3 Dup & 5967003734 & 0 & TGA-03 & SOLID & 7.09 & 5.26 & M/A. & $x$ \\
\hline 96000979 & $s-109$ & 4 SAMPLE & \$967003759 & 0 & TGA-03 & SOL1D & $N / A$ & 11.03 & & $x$ \\
\hline 96000979 & $s-109$ & 5 DUP & s96r003759 & 0 & TEA-03 & SOLIO & 11.03 & 10.44 & H/A & $x$ \\
\hline
\end{tabular}

\section{Final page for worklist \# 11163}

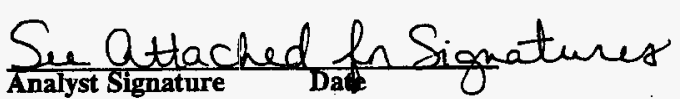

Verifual / Validated by

Blandina
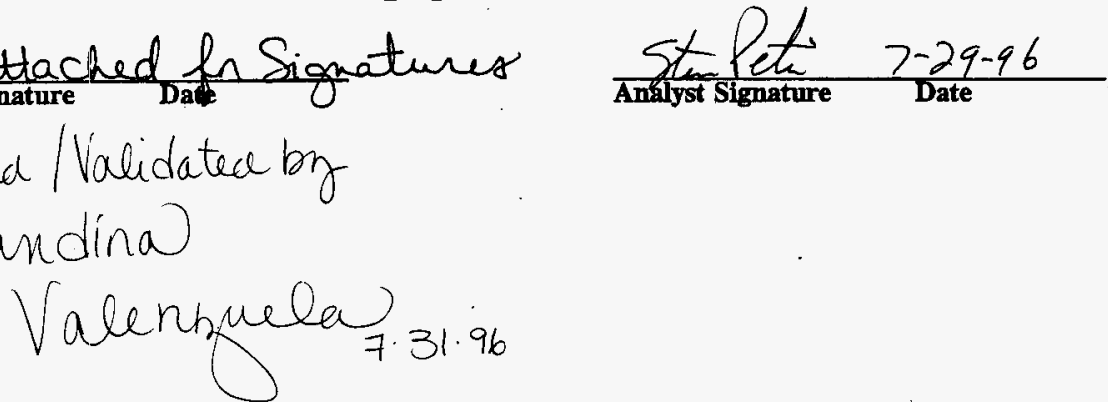

Date Entry Comments:

Uniss shown for QC (SPK \& STD) may not reflect the actual units. $D L=$ Detection Limit, $S=$ Worklist Slot Number, $R=$ Replicate Nimber, $\boldsymbol{A}=$ Aliquot Code. 


\section{LABCORE Data Entry Template for Worklist\#}

\section{Analyst: K⿸尸 Instrument: TGA0 3 Book \# $82 N \& A$}

Method: LA-560-112 Rev/Mod C-1

Worklist Comment: S-109 TGA, RUN UNDER N2. RCJ

\begin{tabular}{|c|c|c|c|c|c|c|c|c|c|c|}
\hline \multirow[t]{2}{*}{ GROUP } & PROJECT & S TYPE & SAMPLE\# & RA & - & MATRIX & ACTUAL & FOUND & $\mathrm{DL}$ & UNIT \\
\hline & & 1 STD & & & TGA-01 & SOLID & & & $N / A$ & $x$ \\
\hline 96000979 & $s-109$ & 2 SNMPLE & 5961003734 & 0 & TGA-01 & SOLID & $N / A$ & & & $x$ \\
\hline 96000979 & $s-109$ & 3 DuP & 5967003734 & 0 & TGA-01 & SOLID & & & N/A & $x$ \\
\hline 96000979 & s-109 & 4 SAMPLE & 596T003759 & 0 & TGA-01 & SOL.ID & N/A & & & $x$ \\
\hline 96000979 & $s-109$ & 5 DuP & \$96T003759 & 0 & TGA-01 & SOLID & & & $N / A$ & $x$ \\
\hline
\end{tabular}

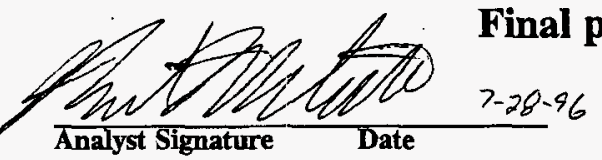

Analyst Signature Date

\section{Final page for worklist \# $\quad 11163$}

\section{Analyst Signature Date}

\section{TGA-03 hrstriment was lised.}

Data Entry Comments:

Units shown for QC (SPR \& STD) may not reflect the actual units. $D L=$ Detection Limit, $S=$ Worklist Slot Number, $R=$ Replicate Number, $A=$ Aliquot Code. 
Curve 1: TEA

F1le Info: TER072e01 sun Jul 28 05: 16:07 1996

Sample Ne1ght: 21.803

TGA STO BeNo-A

ing

\begin{tabular}{l} 
COMPLETEO/VERIFIED THE CALIBRATION/ANALYSIS ON PAGES 107 TO $/ 1 /$ \\
\hline
\end{tabular}

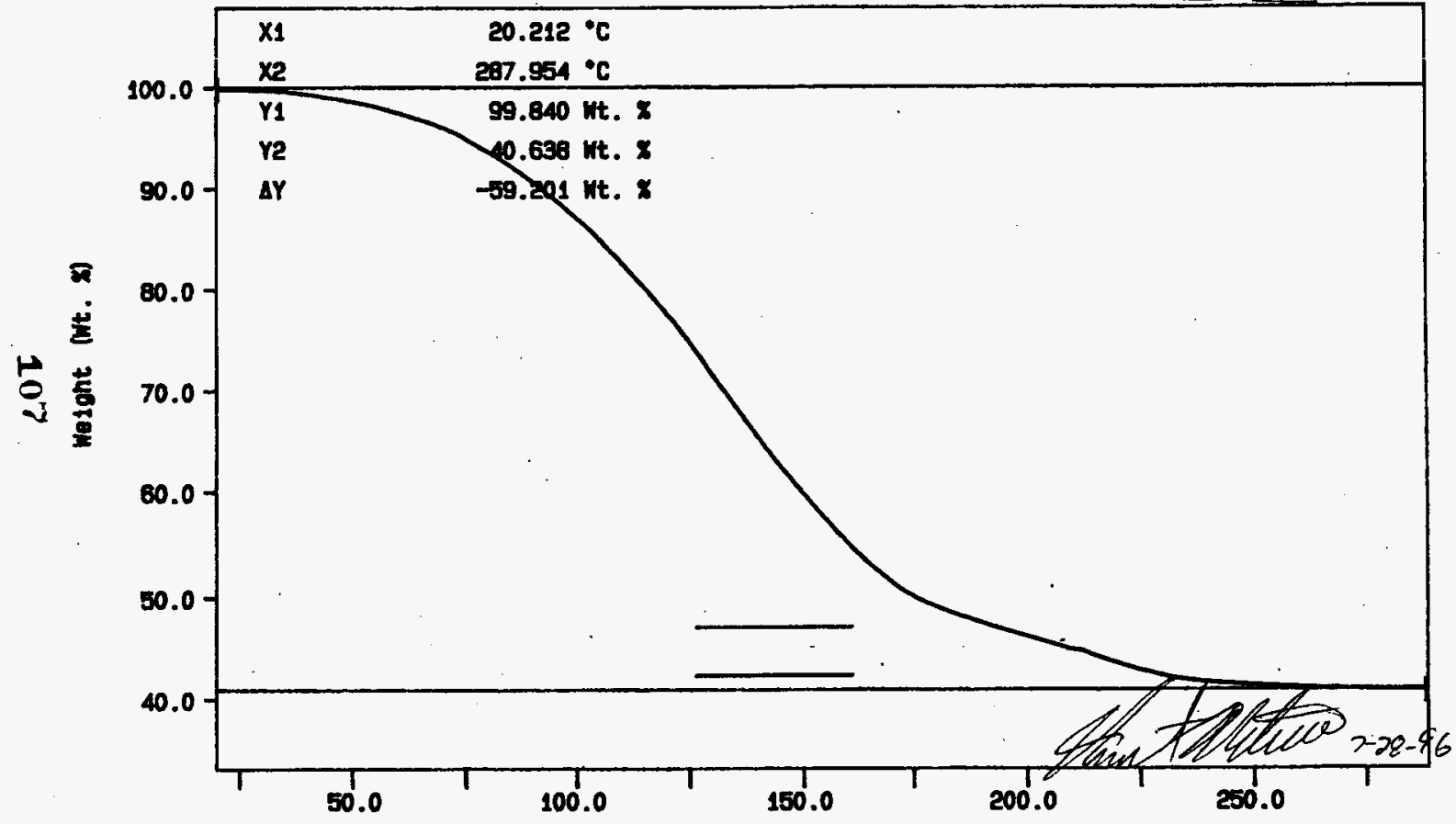

Ne 10C/MIN

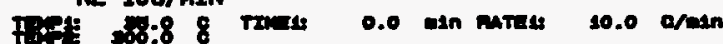

Temperature ("C) KR MONTEITH

PERTIN-EITA

7 Series Therwal Analyeis Syeten 
F110 Info: s96T072004 Sun tul 28 10: 11: 051996

Sanple Weight: 21.035

s96T003734

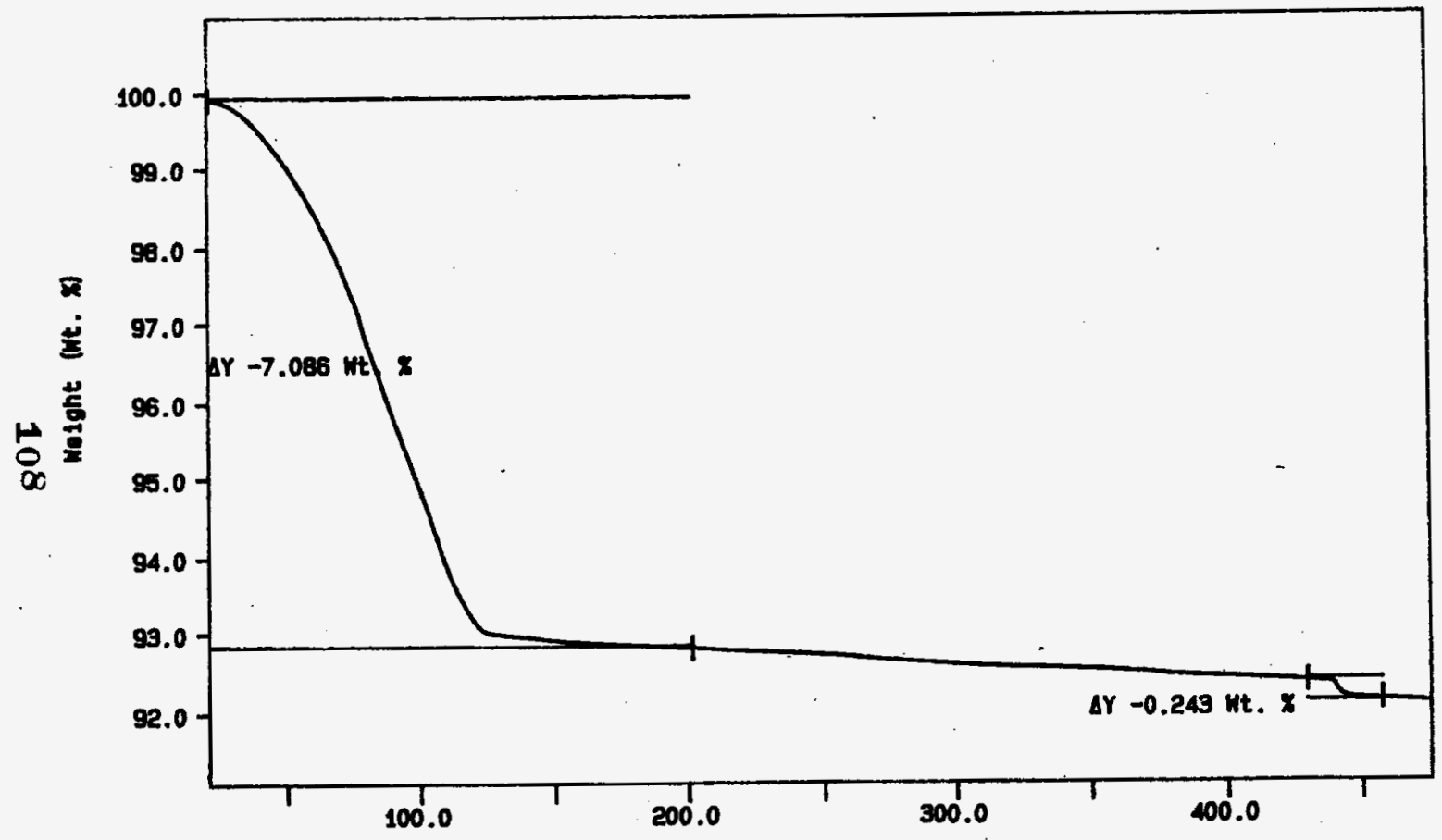

10C/MIN Ne Ft:

0.0 oan mares 10.0 o/man Temperature ('C) KA MONTEITH 
Curve 1: TEA

F11e info: S4mo72805 Sun wul 29 12:40:30 1996

Sanple Welght: 15.065

S95T003734 DUP

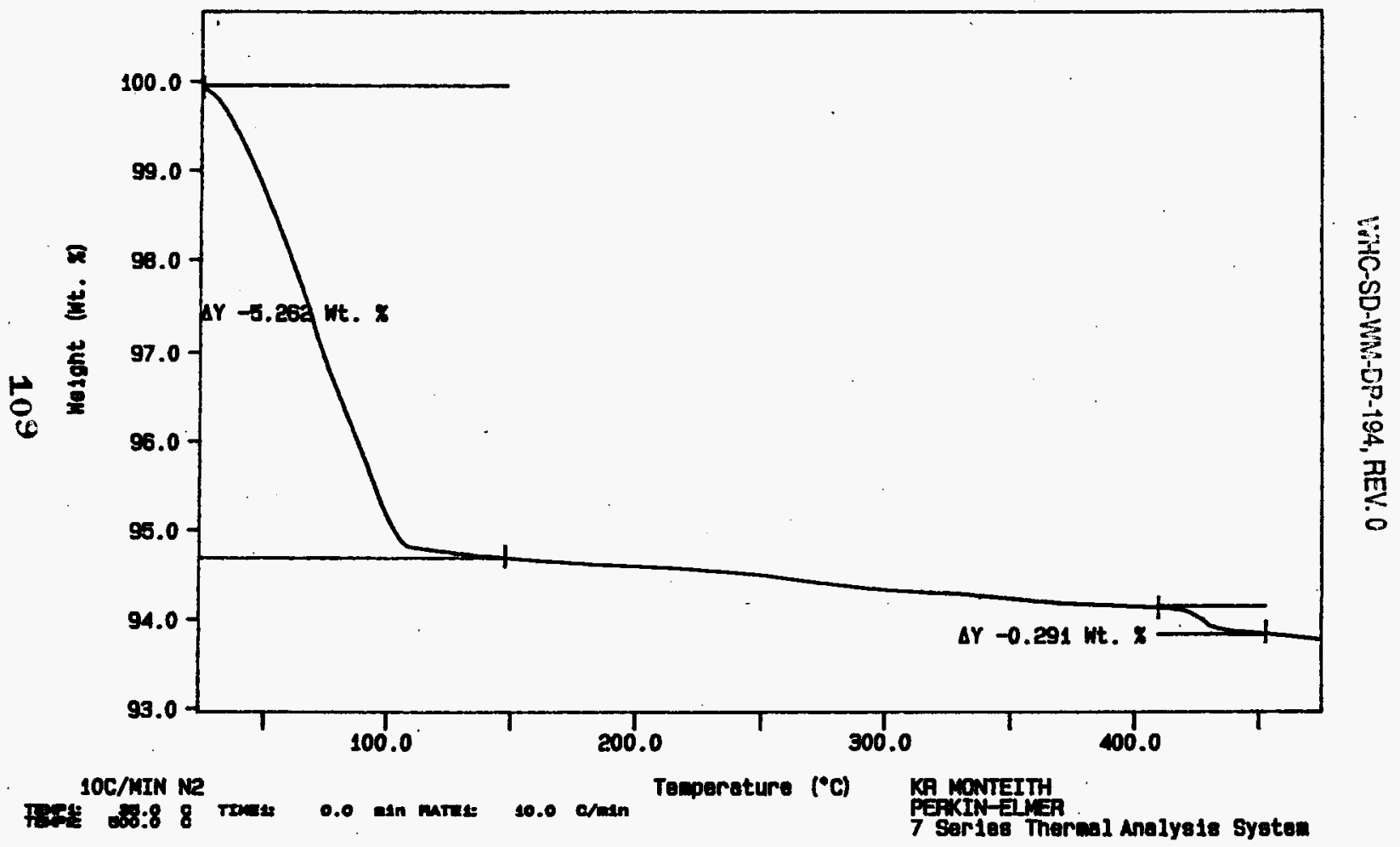


Curve 1: TeA

F11e info: SAw072802 SUn w1 28 06: 30:04 1996

Samle Weight: 26.473 a

S96T003759

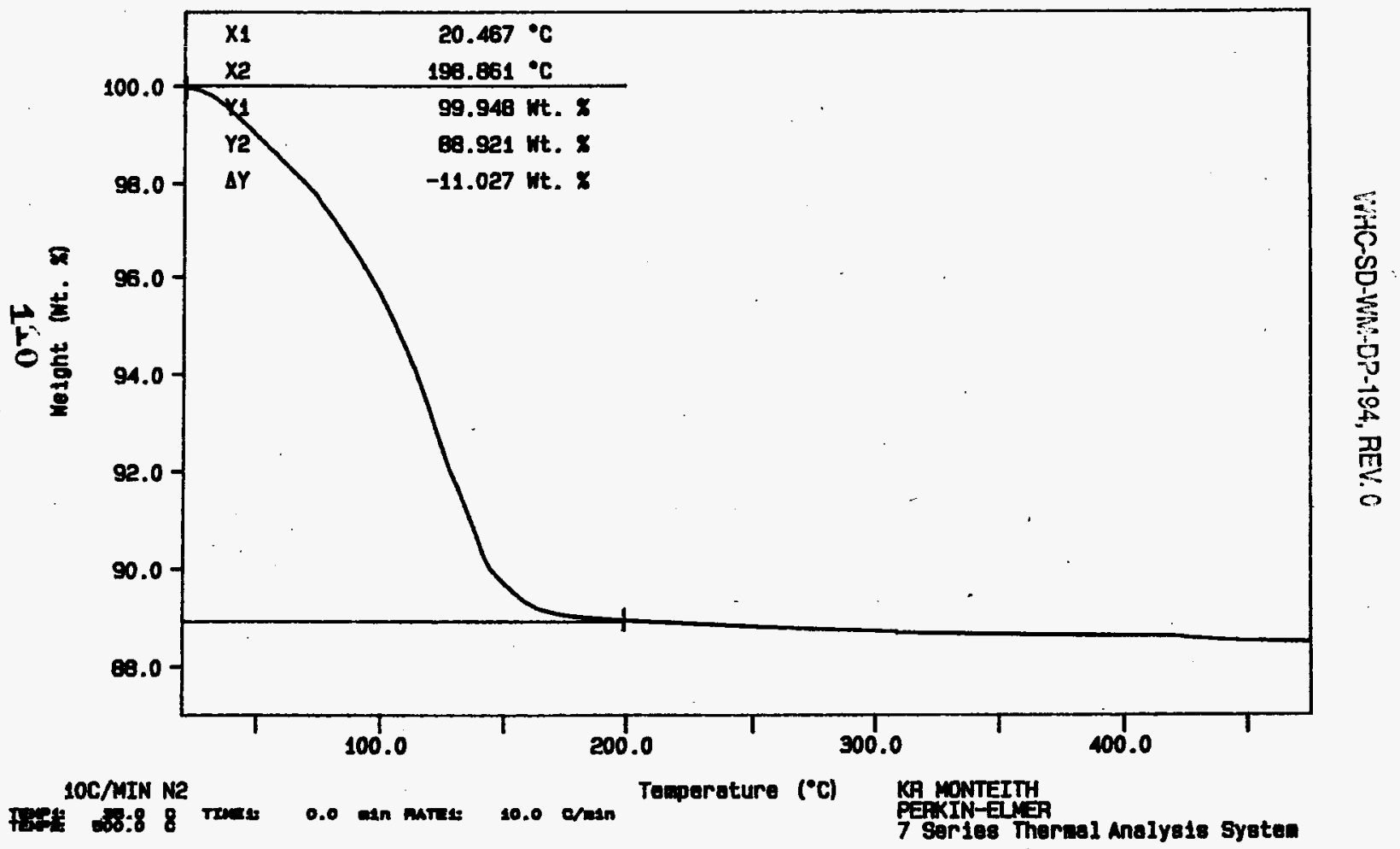




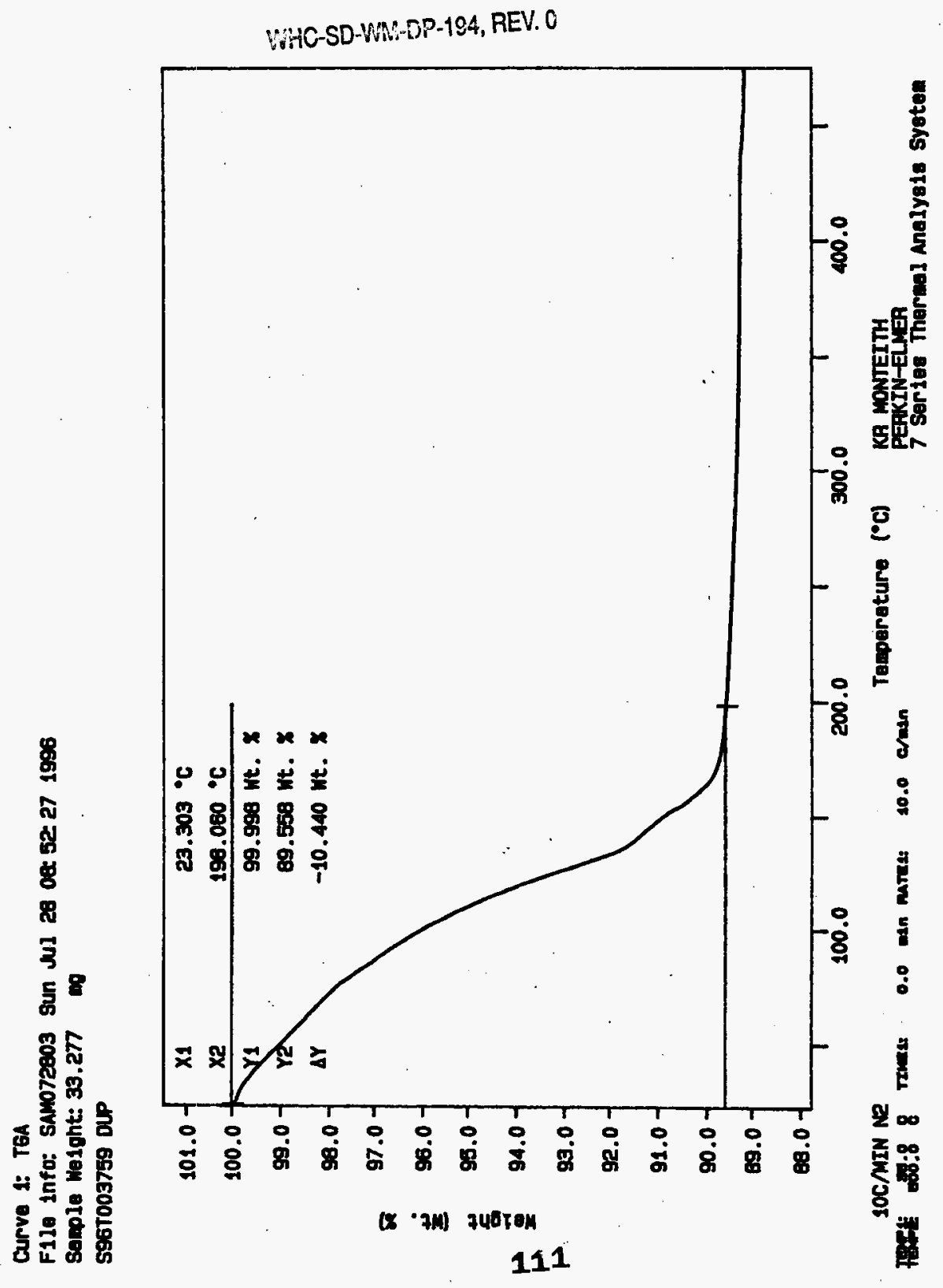


worktistrpt Version $2.105 / 15 / 95$

WHC-SD-VI: SP -194, REV. 0

Page: 1 07/25/96 13:19

LABCORE Data Entry Template for Worklist\#

11164

Analyst: KRM Instrument: TGAO I Book \# $82 N 8 A$

Method: LA-560-112 Rev/Mod

$B-1$

Worklist Comment: S-109 TGA, RUN UNDER N2. RCJ

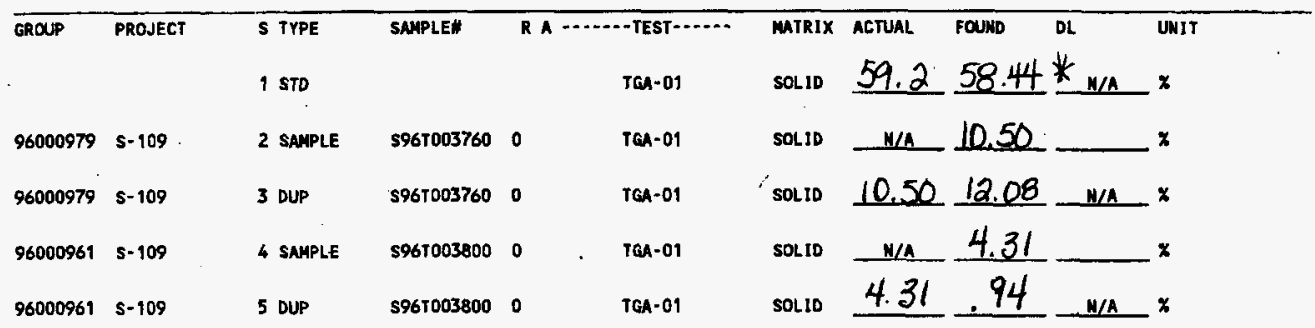

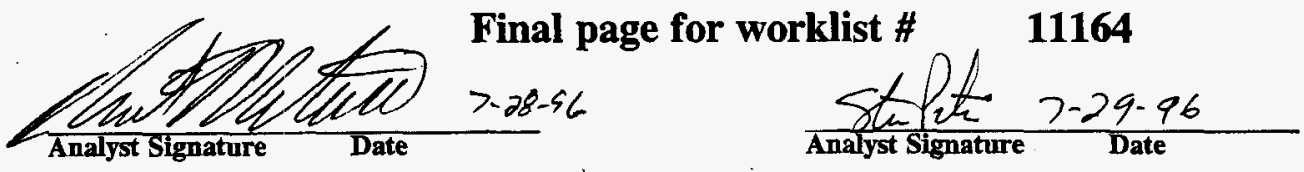

Veritied/Validatea by

Blandina Valenzuela 8-1-96

Data Entry Comments:

Units shown for $Q C$ (SPK \& STD) may not reflect the actual units. $D L=$ Detection Limit, $S=$ Worklist Slot Number, $R=$ Replicate Number, $A=$ Aliquot Code.

112 


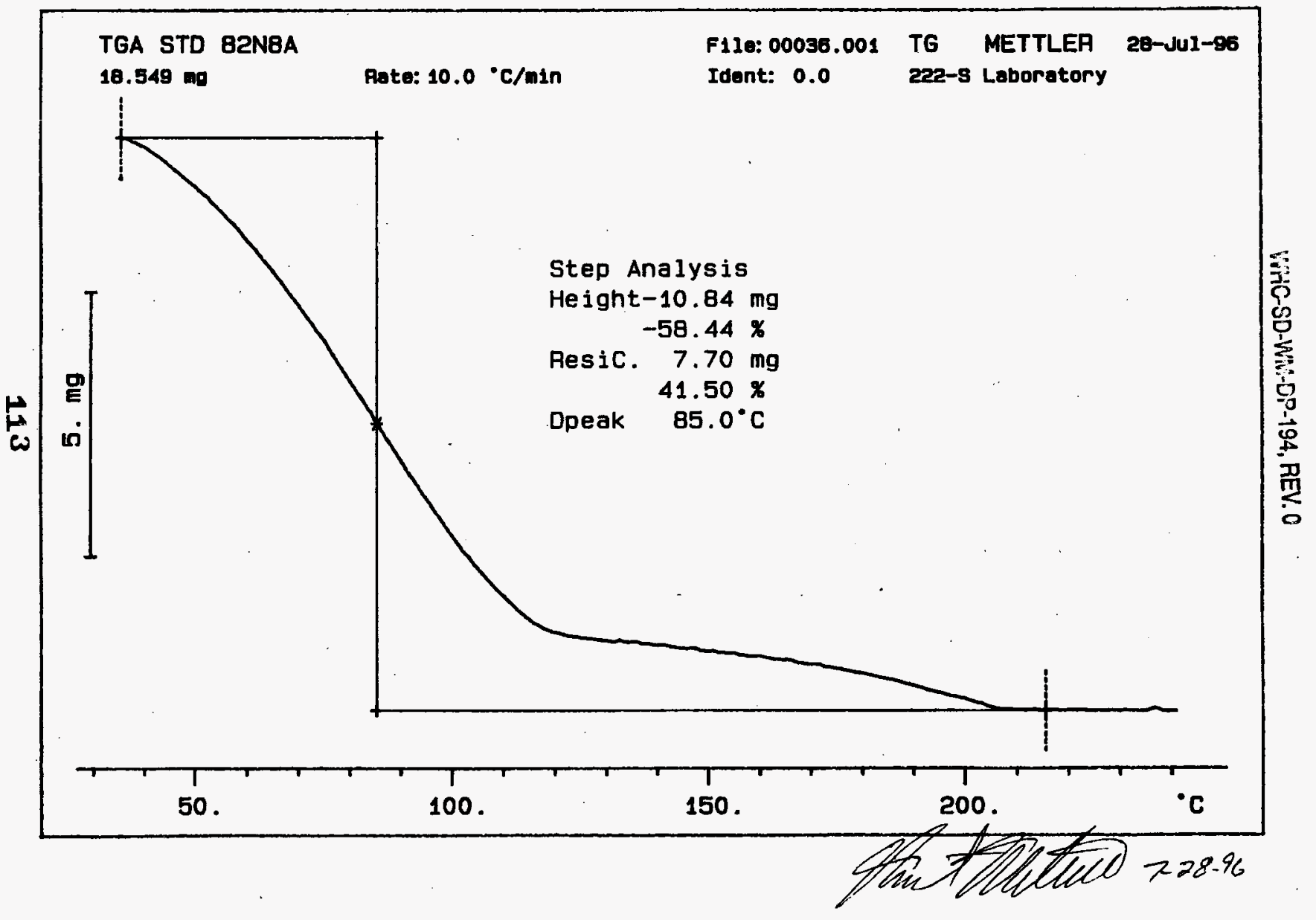




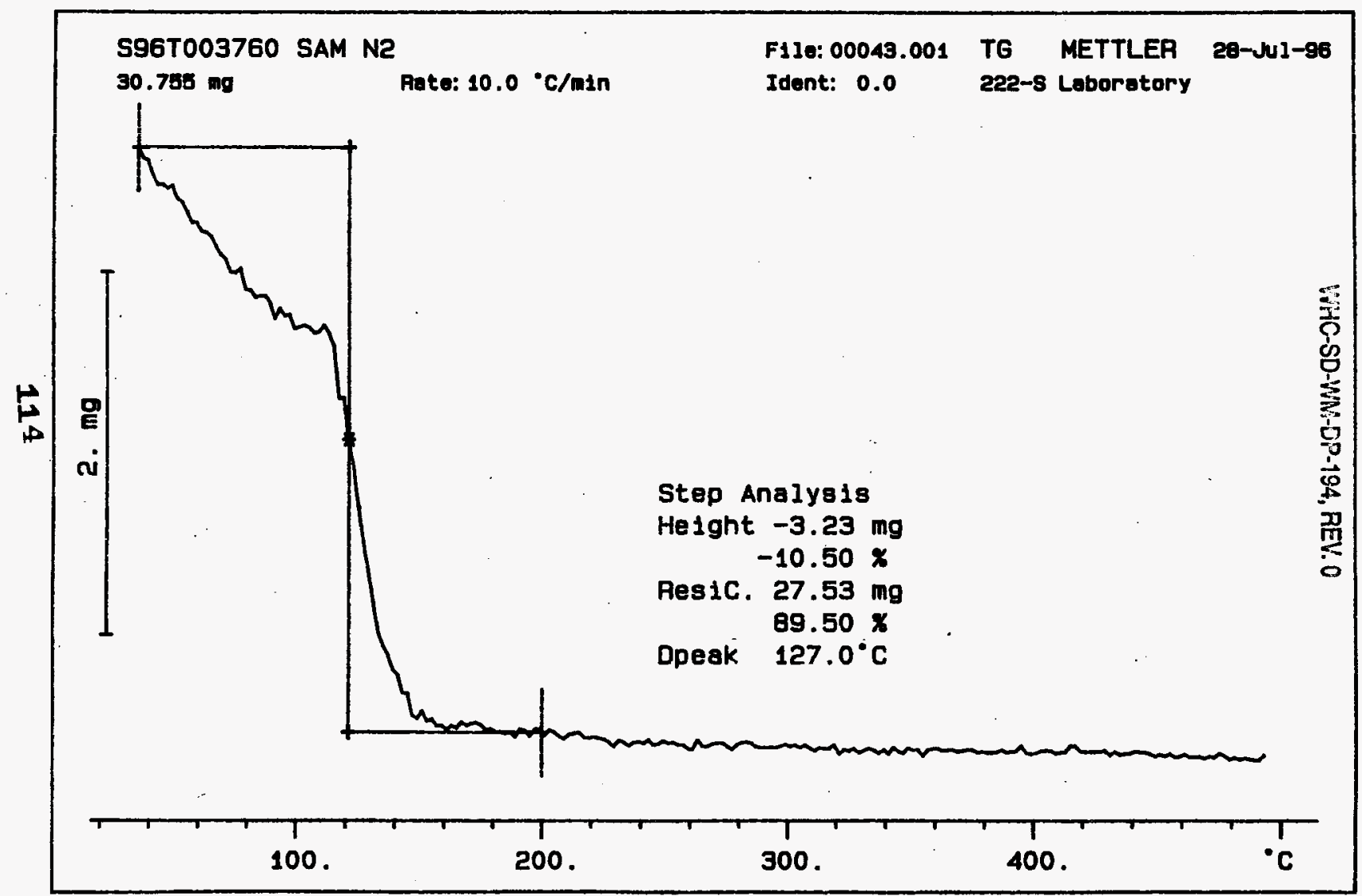




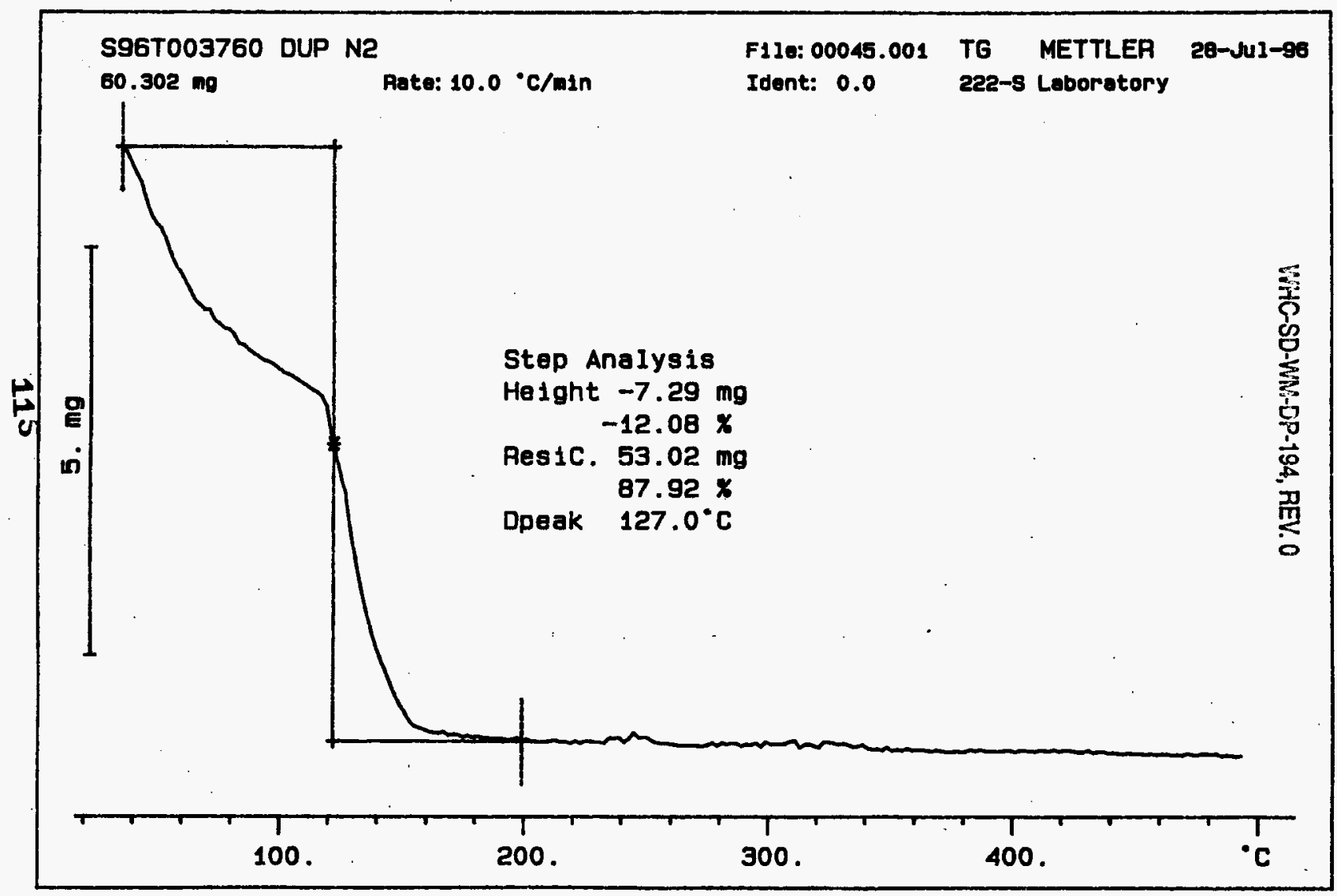




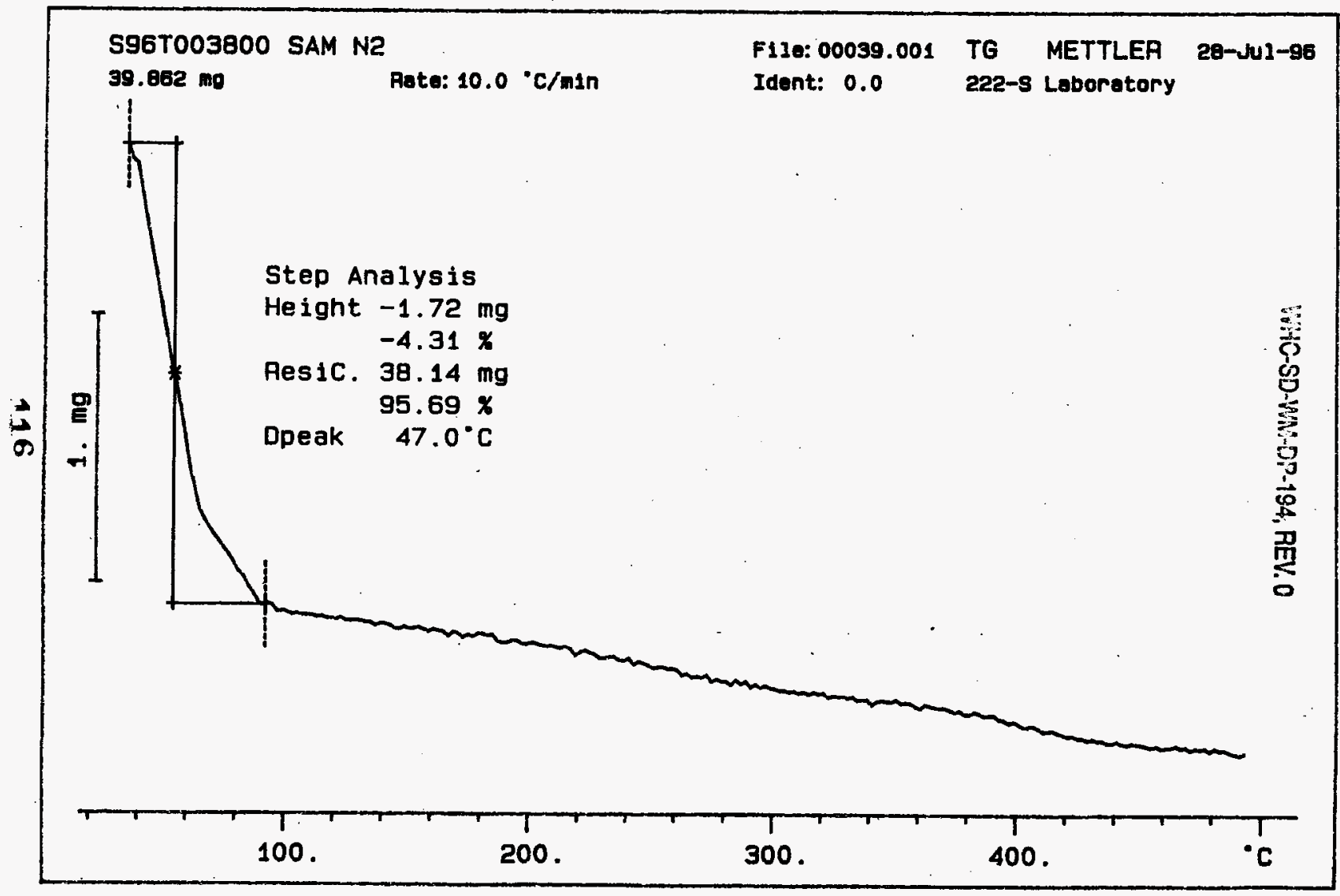




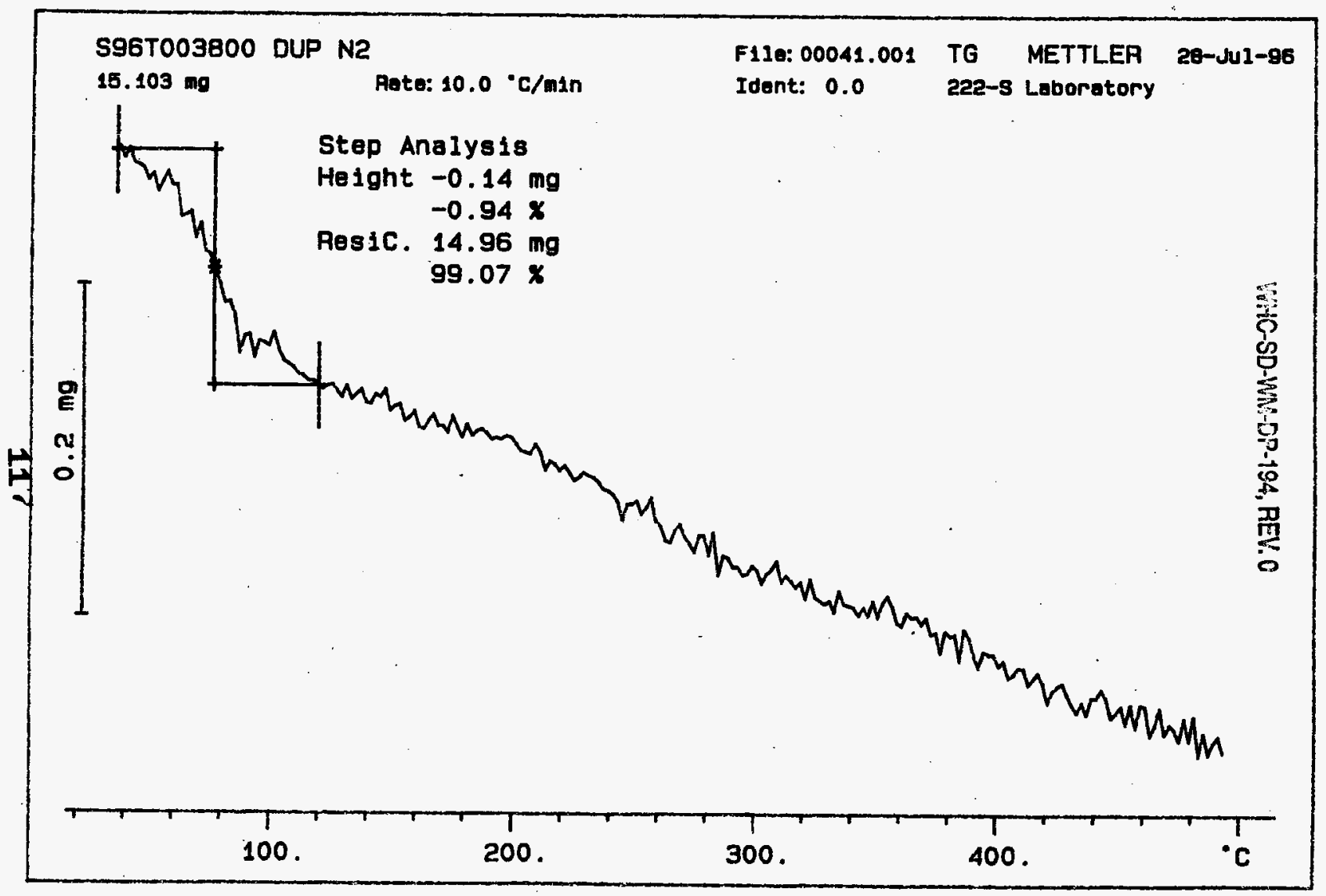




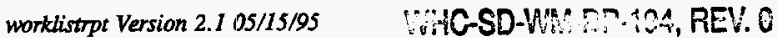

Analyst: KRM Instrument: TGAO L Book \# $82 N 8 \mathrm{~A}$

Method: LA-560-112 Rev/Mod B-1

Worklist Comment: S-109 TGA, RUN UNDER N2. RCJ

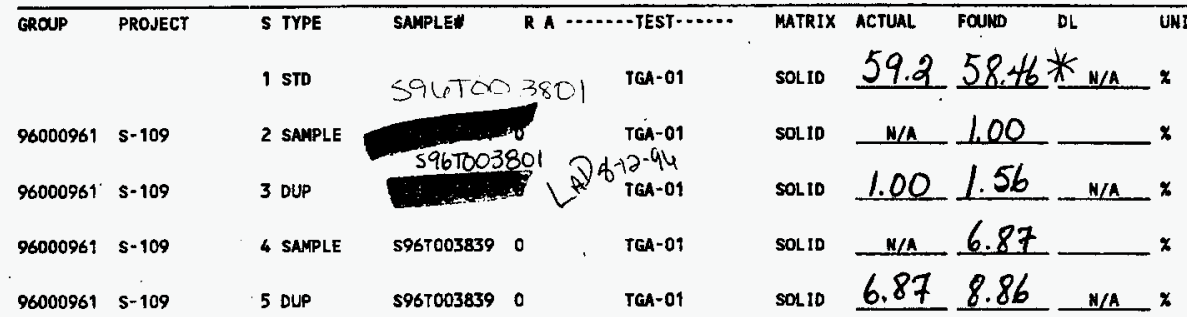

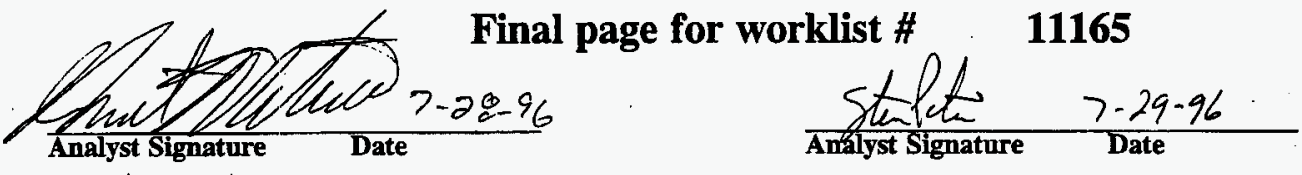

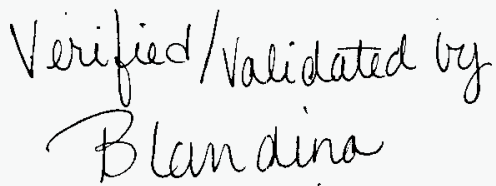

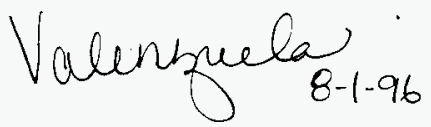

0

Dato Entry Comments:

Units shown for QC (SPK \& STD) may not reflect the actual units. $D L=$ Detection Limit, $S=$ Worklist Slot Number, $R=$ Replicate Number, $A=$ Aliquot Code. 


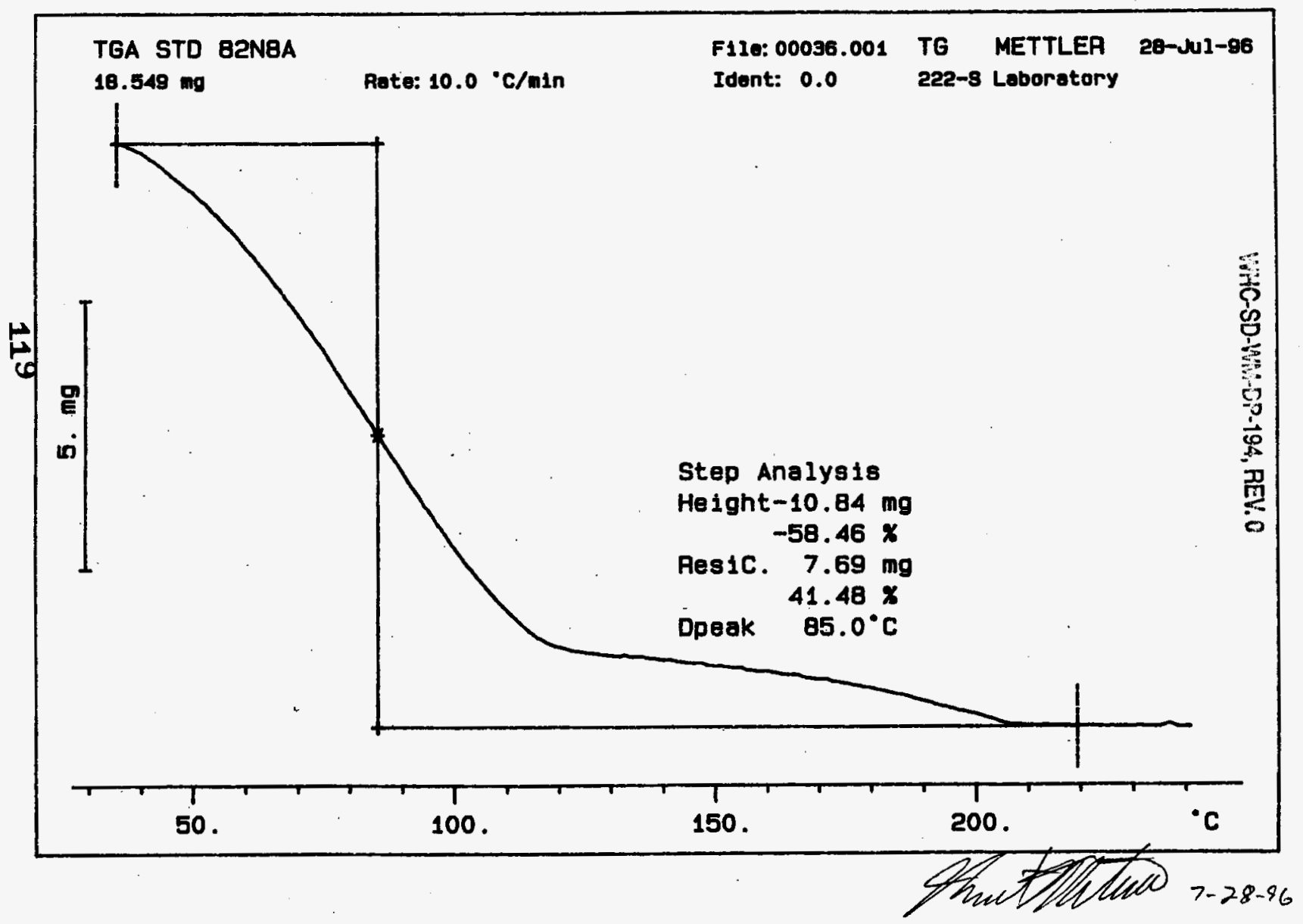




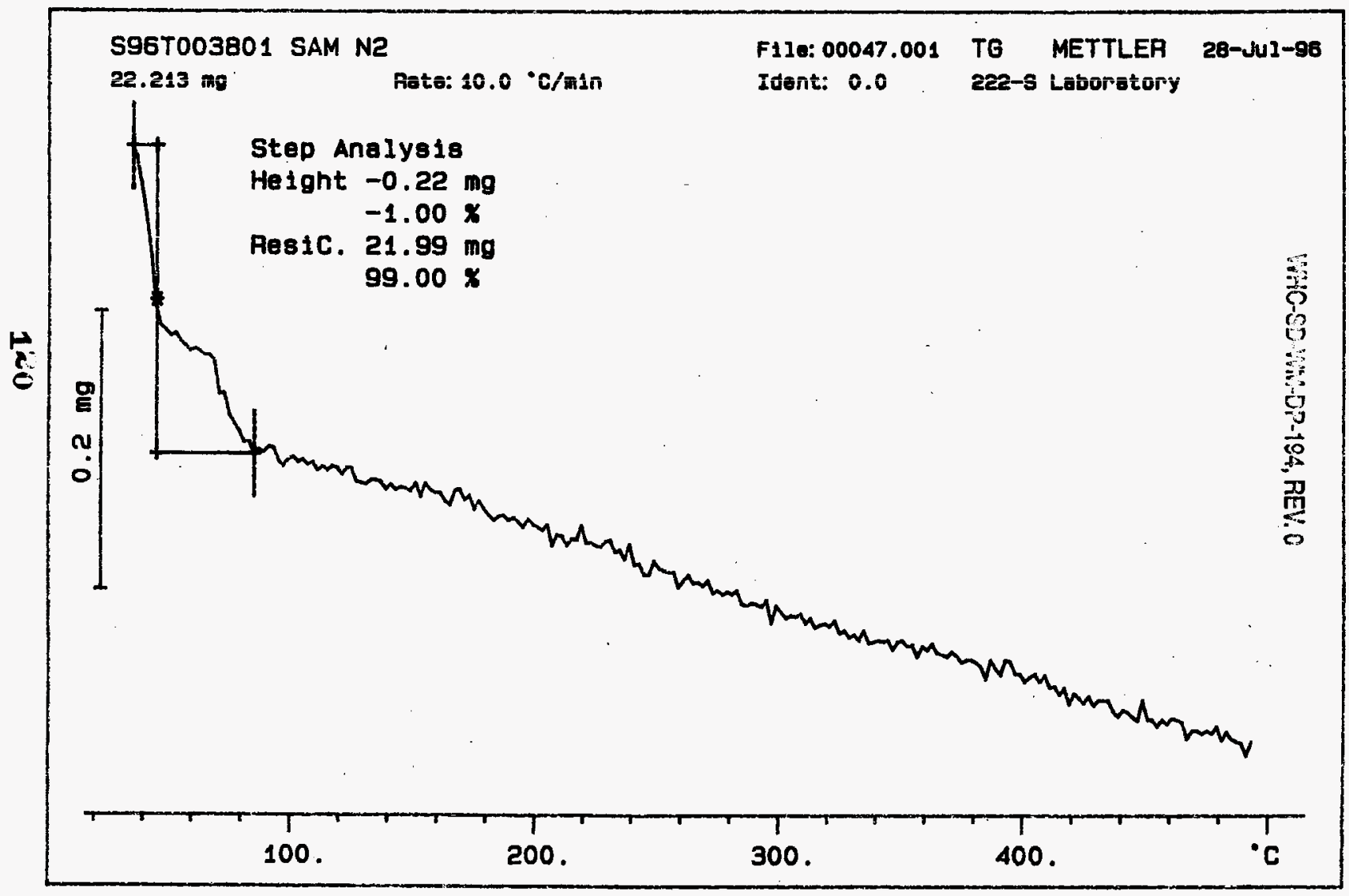




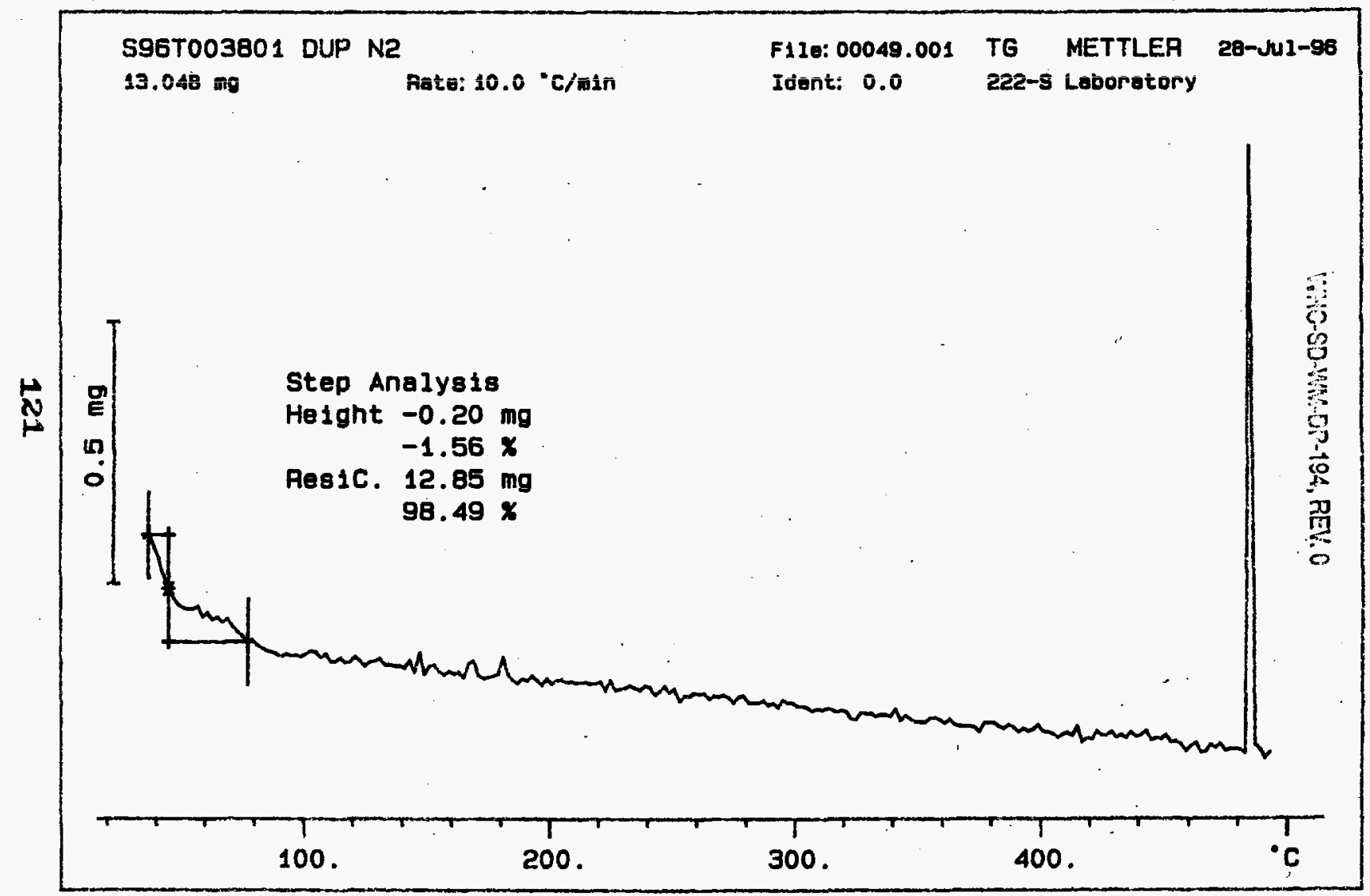




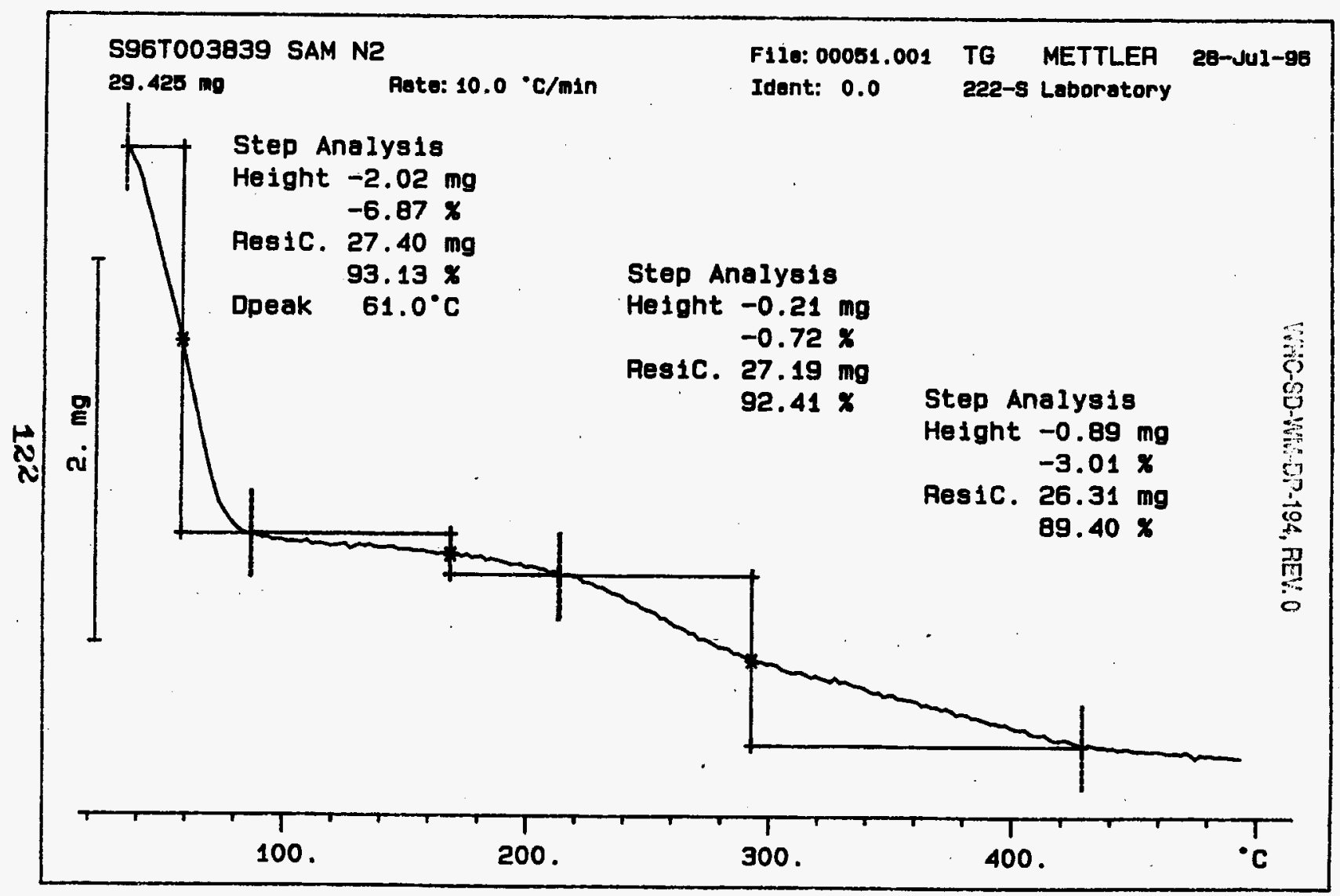




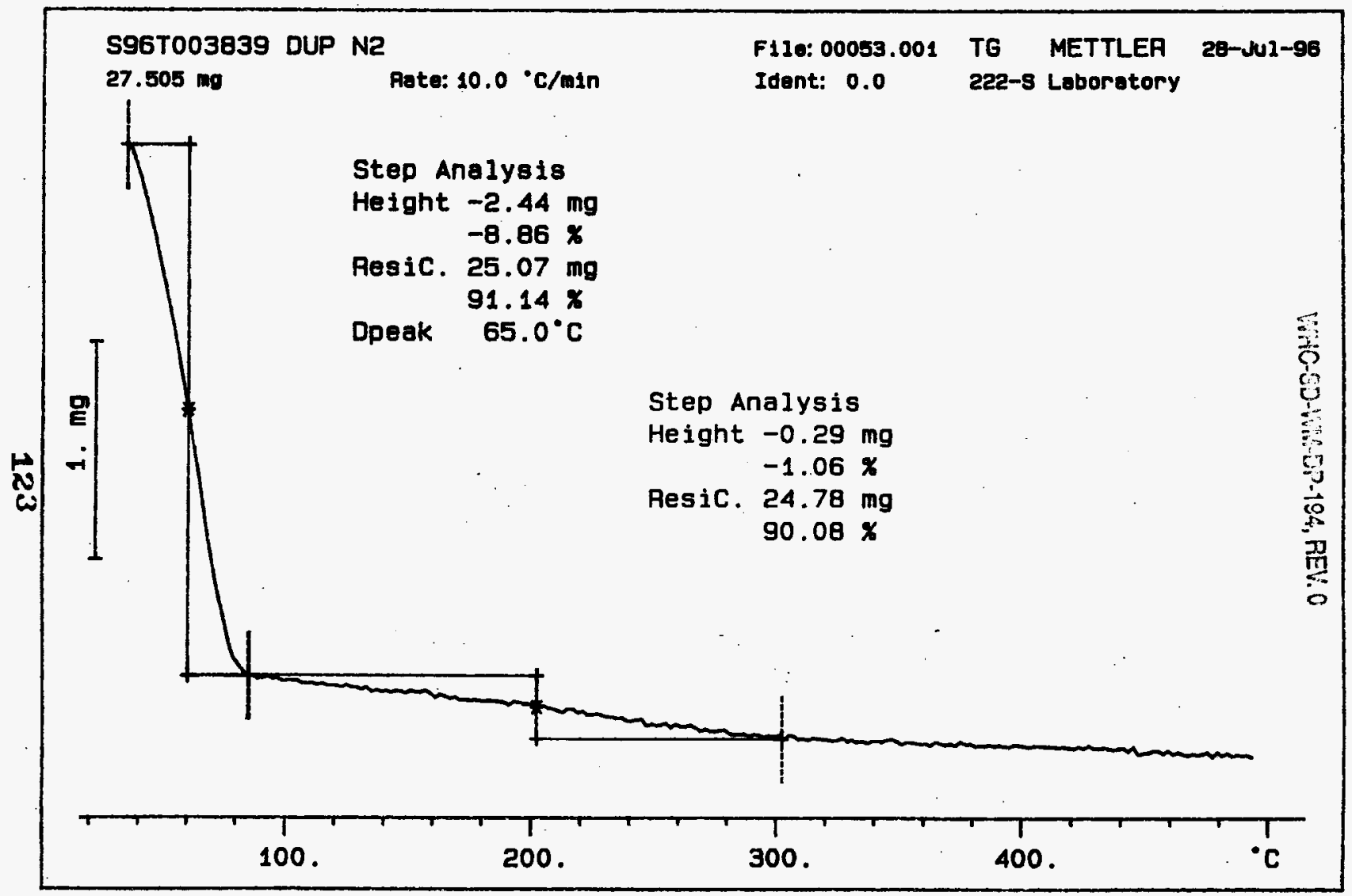


Analyst: KRM Instrument: TGA0 3 Book \# 82N8A

Method: LA-514-114 Rev/Mod C-L

Worklist Comment: S-109 TGA, RUN UNDER N2. RCJ

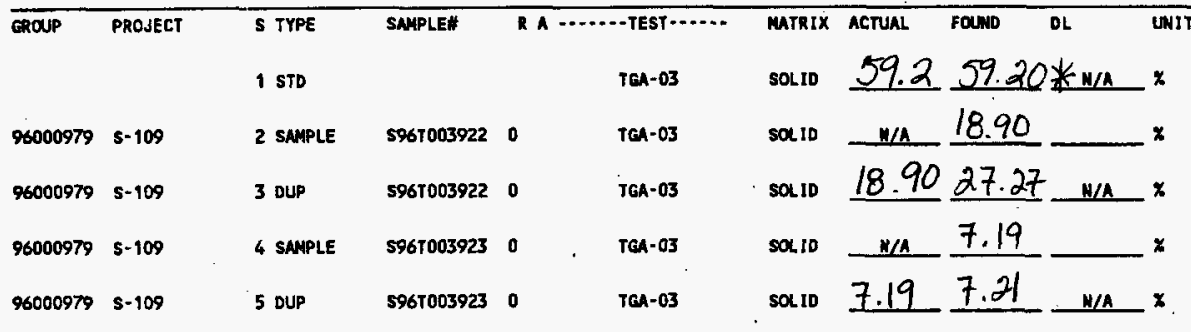

\section{Final page for worklist \# 11166}

Ie attached for siomatures Analyst Signature Date 7-29-96 Verified/Validated by or
Plandina
\[ \text { alenzue } \frac{0}{7.31 .96} \]

Data Entry Comments:

Units shown for $Q C$ (SPR \& STD) may not reflect the actual units. $D L=$ Detection Limit, $S=$ Worklist Slot Number, $R=$ Replicate Number, $A=$ Aliquot Code. 


\section{LABCORE Data Entry Template for Worklist\#}

Analyst: $-K \mathrm{KM}$ Instrument: TGA0 3 Book \# $8208 \mathrm{~A}$

Method: LA-560-112 Rev/Mod

Worklist Comment: S-109 TGA; RUN UNDER N2. RCJ

\begin{tabular}{|c|c|c|c|c|c|c|c|c|c|c|}
\hline \multirow[t]{2}{*}{ GROUP } & \multirow[t]{2}{*}{ PROJECT } & \multirow{2}{*}{$\begin{array}{l}\text { S TYPE } \\
1 \text { sTd }\end{array}$} & SAKPLE\# & \multicolumn{2}{|c|}{ R A $\cdots+-$ TEST $\ldots \ldots$} & \multirow{2}{*}{$\begin{array}{l}\text { MAtRIX } \\
\text { SOLID }\end{array}$} & \multirow[t]{2}{*}{ ACTUAL } & \multirow[t]{2}{*}{ FOUND } & \multirow[t]{2}{*}{$\overline{D L}$} & UNIT \\
\hline & & & -1 & 22 & TGA-01 & & & & & $x$ \\
\hline 96000979 & s- 109 & 2 SAMPLE & & 0 & TGA-01 & SOLID & $N / A$ & & & $x$ \\
\hline 96000979 & s-109 & 3 DUP & & 10 & TGA-01 & SOLID & & & $N / A$ & $x$ \\
\hline 96000979 & 5.109 & 4 SNMPLE & \$96T003923 & 0 & TCA-01 & SOLID & $N / A$ & & & $x$ \\
\hline 96000979 & $s \cdot 109$ & 5 DUP & \$96T003923 & 0 & TGA-01 & SOLID & & & $N / A$ & $x$ \\
\hline
\end{tabular}

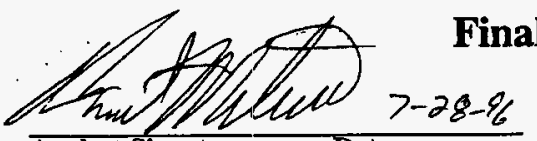

Analyst Signature
Date

\section{Final page for worklist \#}

\section{6}

Units shown for QC (SPK \& STD) may not reflect the actual units. $D L=$ Detection Limit, $S=$ Worklist Slot Number. $R=$ Replicate Number, $A=$ Aliquot Code. 
Curve 1: TEA

File info: TERO72801 Sun wul 28 0x: 16: 071996

Sample We1ght: 21.803

TEA STD 82NG-A

$\operatorname{mog}$

SIGNATURE BELOW REPRESENTS CHEMICA! TECHNOIOGIST/CHEMIST THAT COMPLETED/VERIFIED THE CALIBRAT ION/ANALISIO ON PAGES 126 TO 130.

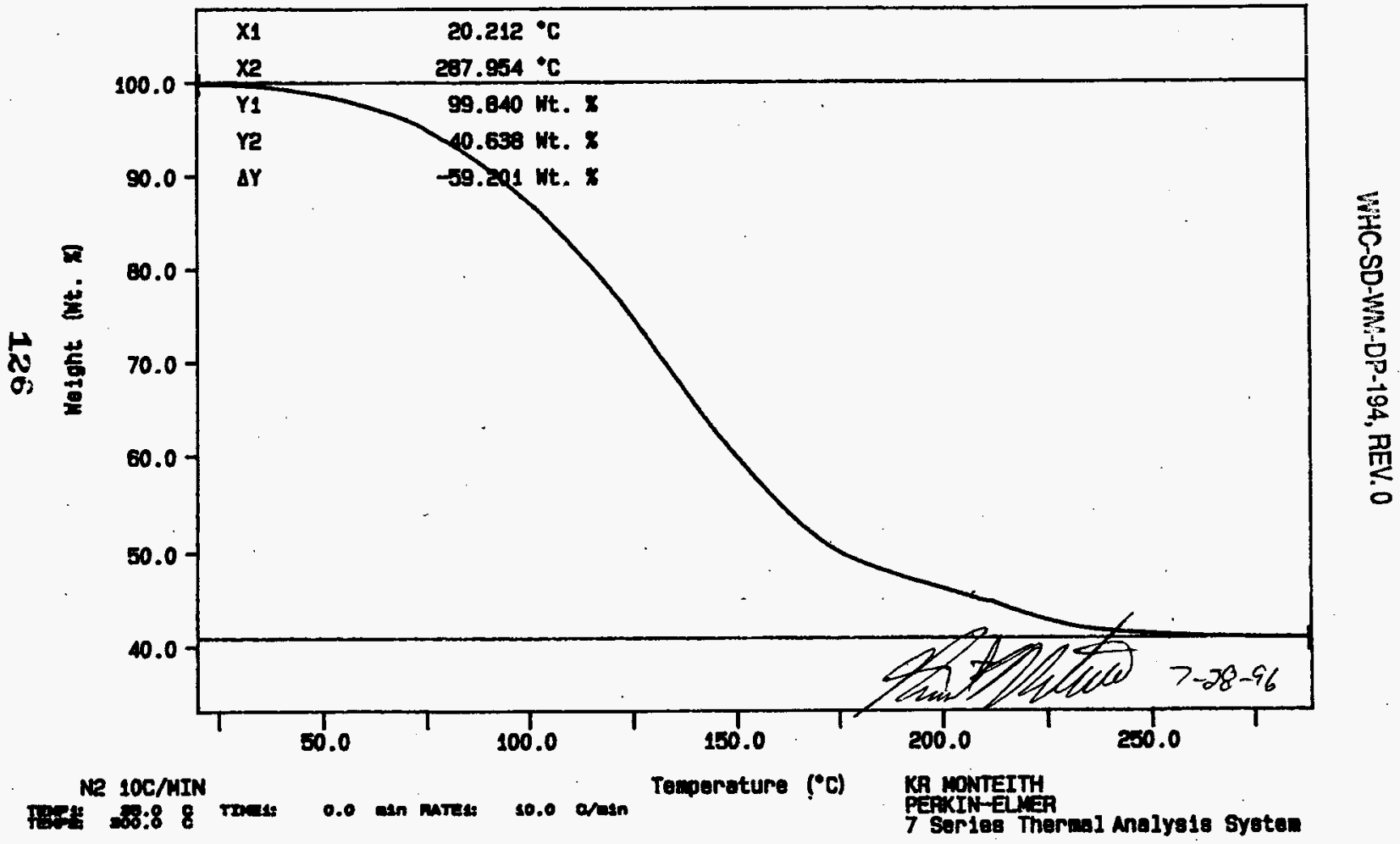


WHiC-SD-WM-DP-194, REV. 0

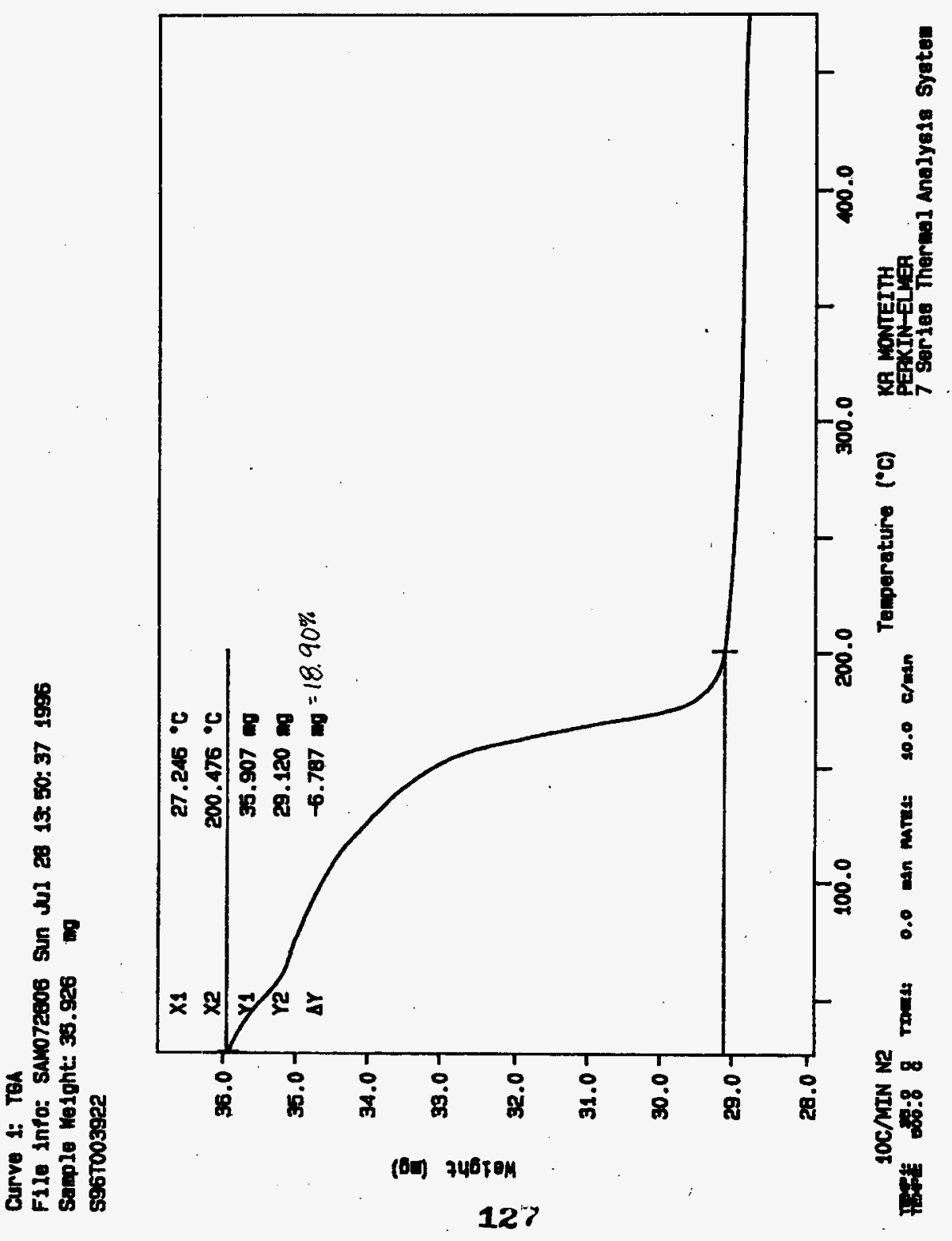


WHC-SD-WM-DP-194, REV. 0

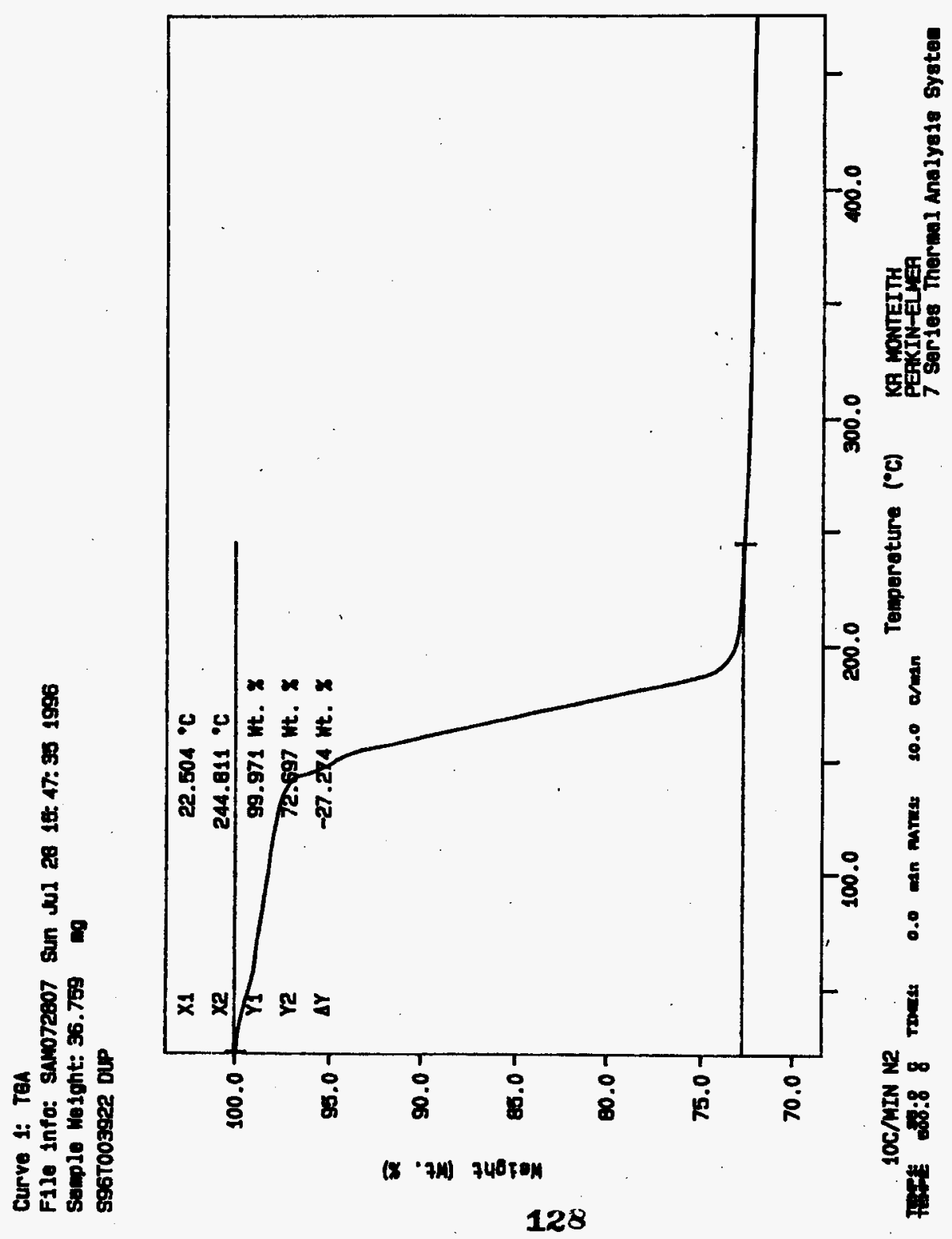


Curve 1: TEA

F110 Info: sumb72:00 sun w1 28 19:07:55 1996

Samle Nelght: $23.243 \mathrm{mg}$

S9oT003923 SAK

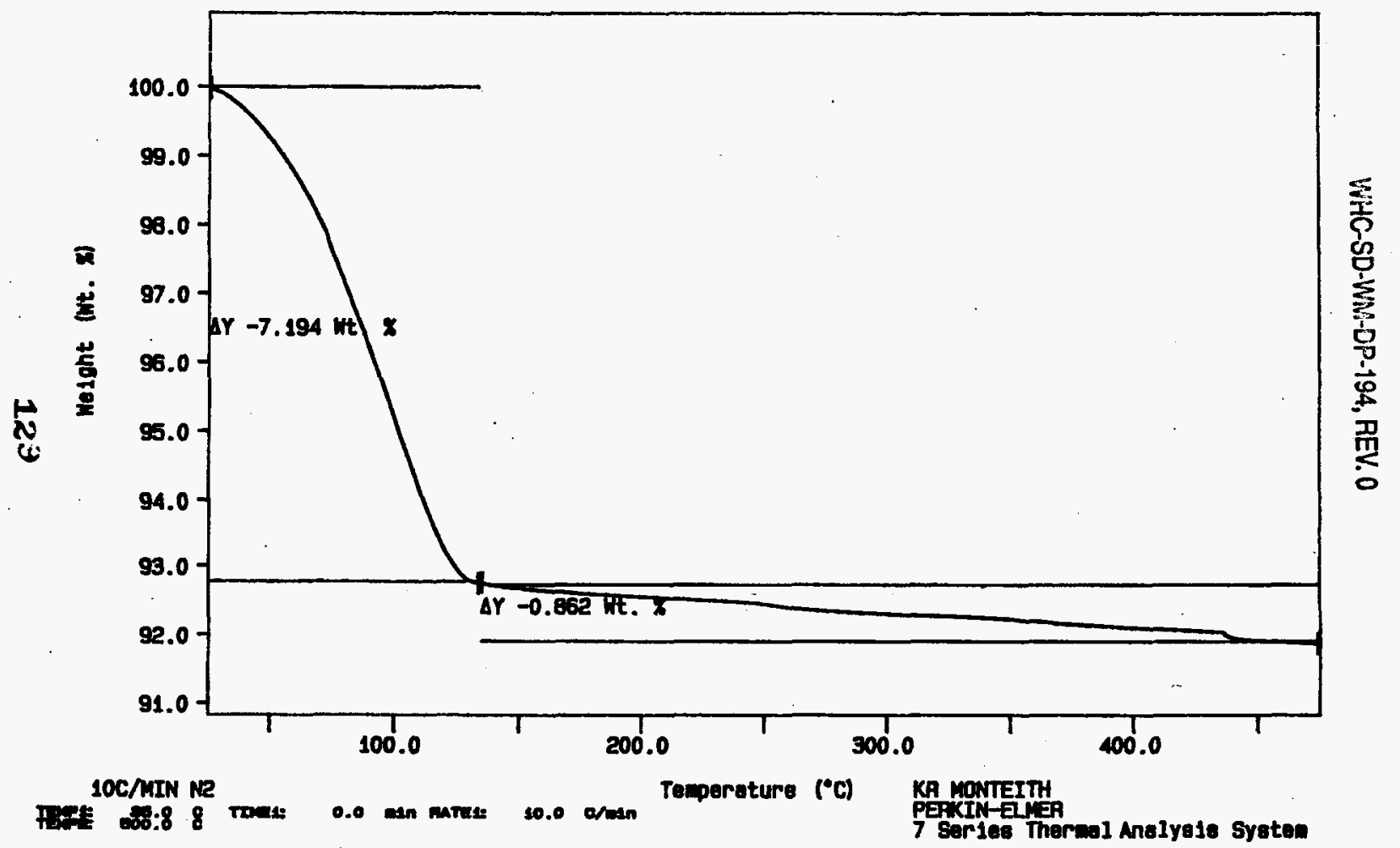


WHC-SD-WMA-DP-194, REV. 0

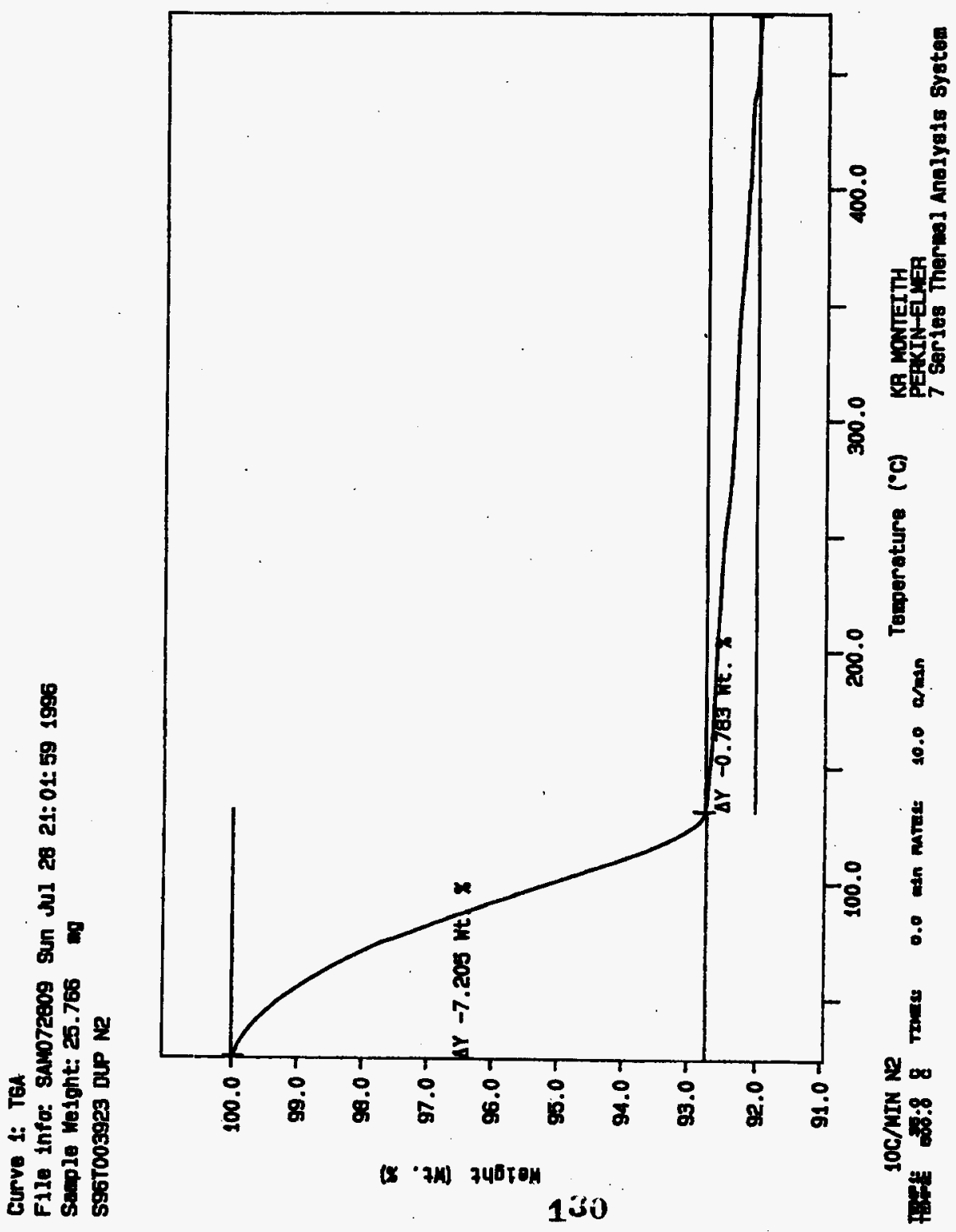


worktistrpt Version 2.1 05/15/95 WHG-SD-WM-D? -104, REV. 0

07/29/96 13:53

LABCORE Data Entry Template for Worklist\#

11167

Analyst: $\quad$ ADP $\quad$ Instrument: TGA0 3 Book * 82N8A

Method: LA-514-114 Rev/Mod C-1

Worklist Comment: S-109 TGA, RUN UNDER N2. RCJ

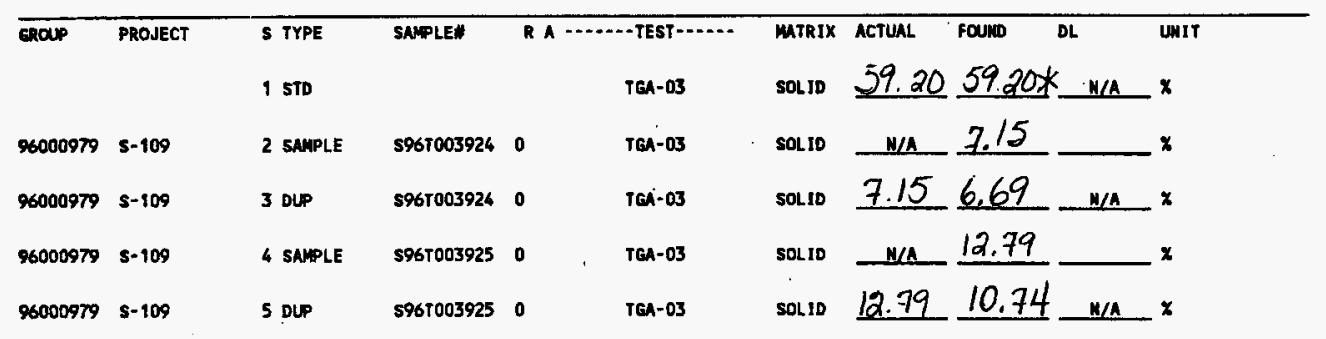

Final page for worklist \# $\quad 11167$

Sol attached $\frac{\text { or signatures }}{\text { Magnate }}$

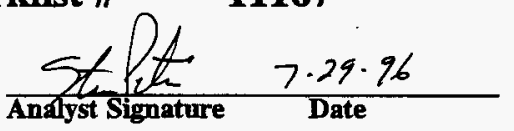

Verified/ Validated by

bor.

Blandina

Valenzuela

$8-1-96$ Data Entry Connors: S967003925 results were the sum of two wight

Units shown for QC (SFK \& STD) may not reflect the actual units. $D L=$ Detection Limit, $S=$ Worklist Slot Number, $R=$ Replicate Number, $A=$ Aliquot Code.

131 


\section{Analyst:}

Instrument: TGA0

Book \# $82 N 8 h$

Method: LA-560-112 Rev/Mod C 1

Worklist Comment: S-109 TGA, RUN UNDER N2. RCJ

\begin{tabular}{|c|c|c|c|c|c|c|c|c|c|c|}
\hline \multirow{2}{*}{ GROUP } & \multirow[t]{2}{*}{ PROJECT } & S IYPE & \multirow[t]{2}{*}{ SNAPLE\# } & \multicolumn{2}{|c|}{ R A $\ldots \ldots$ TEST $\ldots \ldots$} & \multirow{2}{*}{$\begin{array}{l}\text { MATRIX } \\
\text { SOLID }\end{array}$} & \multirow[t]{2}{*}{ ACTUAL } & \multirow[t]{2}{*}{ Fould } & \multirow[b]{2}{*}{$M / A$} & \multirow{2}{*}{$\begin{array}{l}\text { UNIT } \\
x\end{array}$} \\
\hline & & 1 sto & & & TGA-01 & & & & & \\
\hline 96000979 & $s-109$ & 2 SAMPLE & \$96T003924 & 0 & TCA-01 & SOLID & $\mathbf{N} / \mathbf{A}$ & & & $x$ \\
\hline 96000979 & $s-109$ & 3 DUP & S967003924 & 0 & TGA-01 & SOLID & & & W/A & $x$ \\
\hline 96000979 & $5-109$ & 4 SAMPLE & S96T003925 & 0 & TaA-01 & SOLIO & N/A & & & $x$ \\
\hline 96000979 & $s-109$ & 5 DUP & s96r003925 & 0 & TGA-09 & SOLID & & & N/A & $x$ \\
\hline
\end{tabular}

$\underset{\text { Analyst Signature }}{\text { Date }}$

\section{Analyst Signature Date}

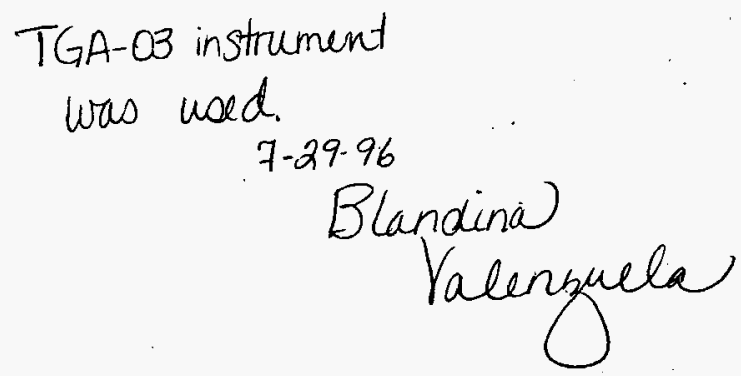

Data Entry Comments:

Units shown for $Q C$ (SPK \& STD) may not reflect the actual units. DL = Detection Limit, $S=$ Worklist Slot Number, $R=$ Replicate Number, $A=$ Aliquot Code. 
Curve 1: TGA

File Info: TER072801 Sun Jul 28 05: 16:07 1996

Sanple Weight: 21.803

TGA STD BENB-A

SIGNATUIRE GELOW REPRESENTS CHEM! A : TECWNOIOGISTICHEMIST THAT

COMPLETED/VERIFIED THE CALIBAAIION AI ALYSIS ON PRGES $/ 33$ TO 137

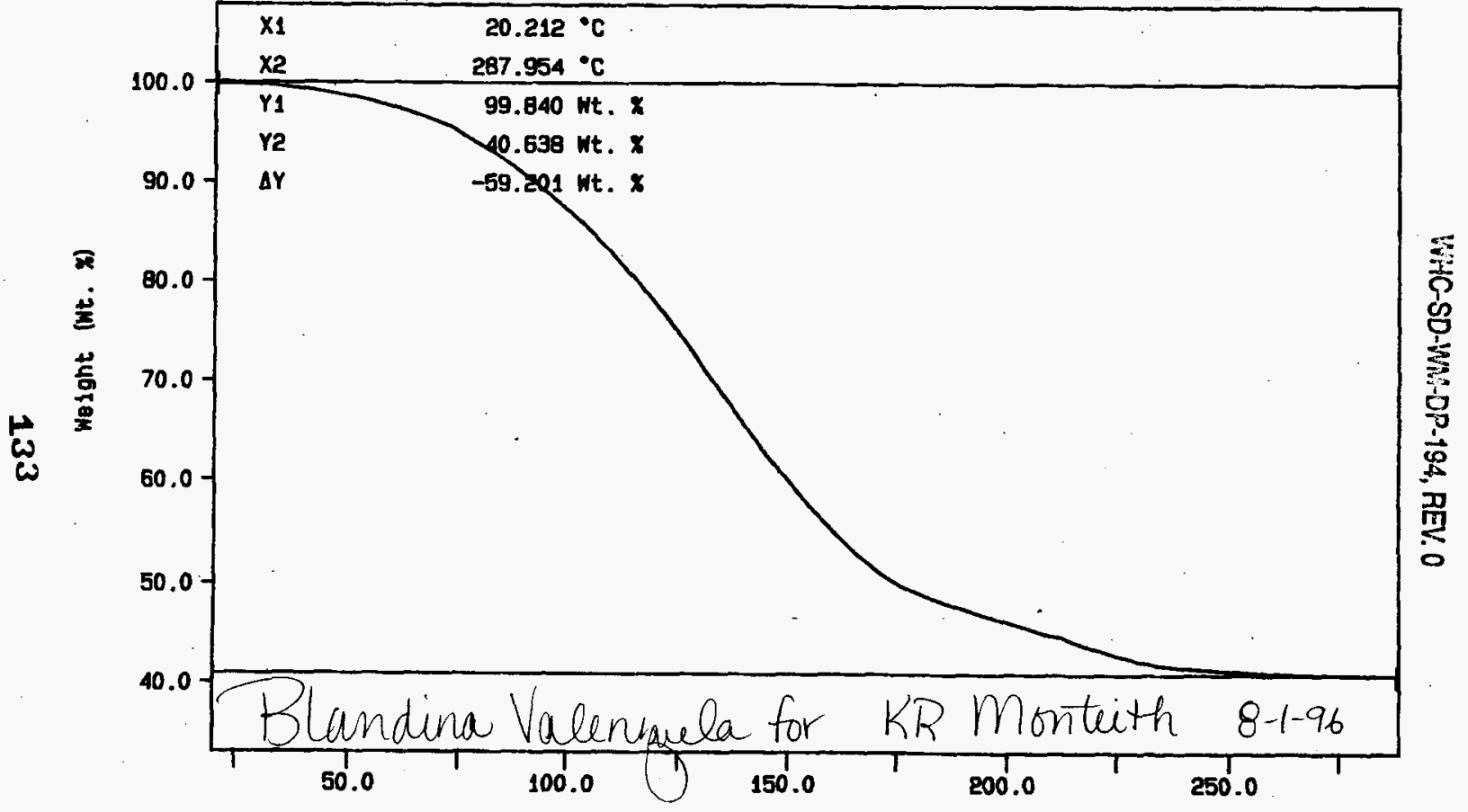

Ne 10C/MIN

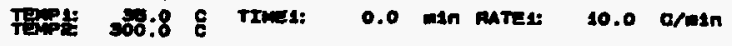

Temperature $\left({ }^{\circ} \mathrm{C}\right) \quad$ KR MONTEITH

PERKIN-ELMEA

7 Series Thermel Anajyois Systen

Mon JuI 29 05: 39:05 1996

$\frac{1}{5}$ 
WHC-SD-WM-OP-194, REV. 0

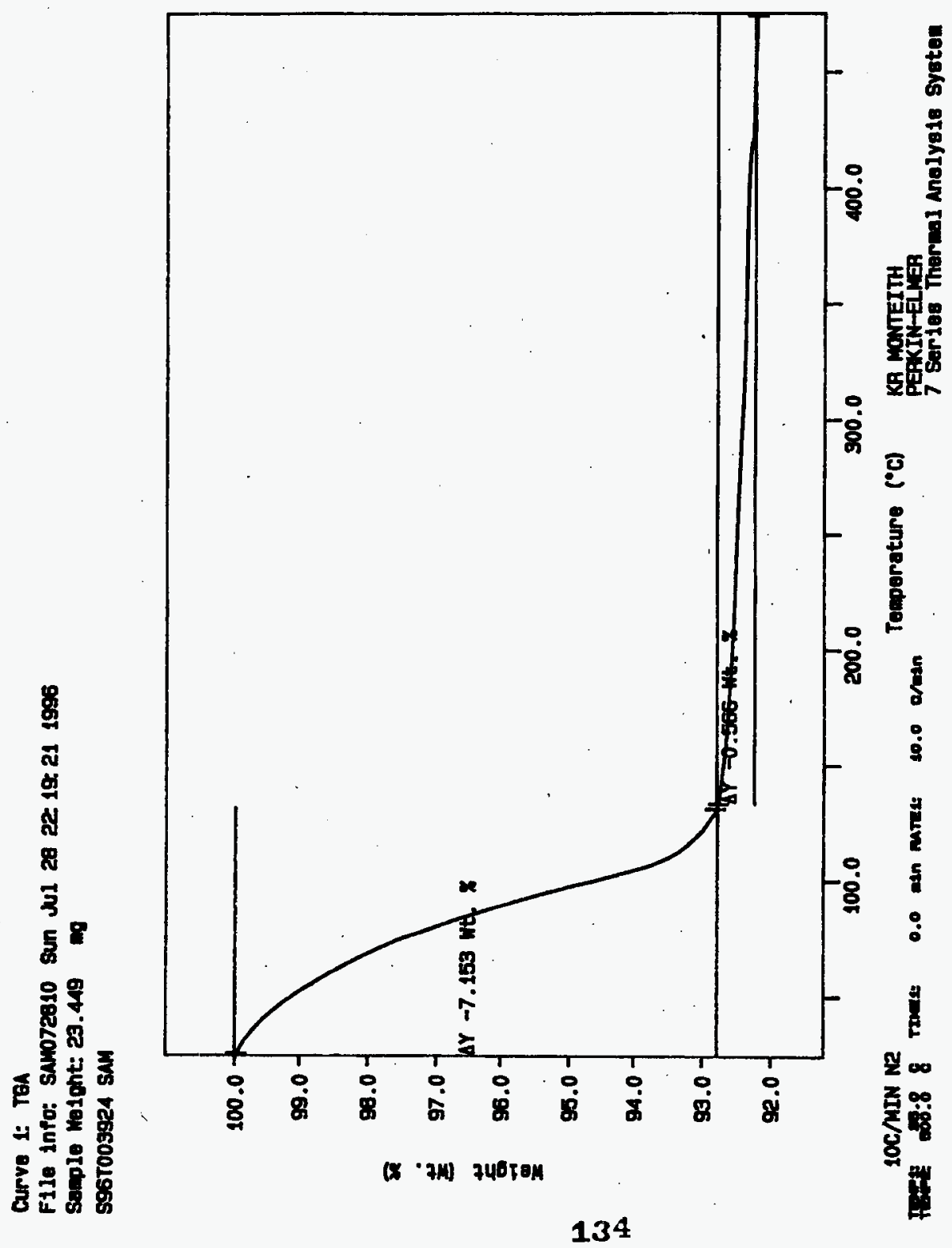




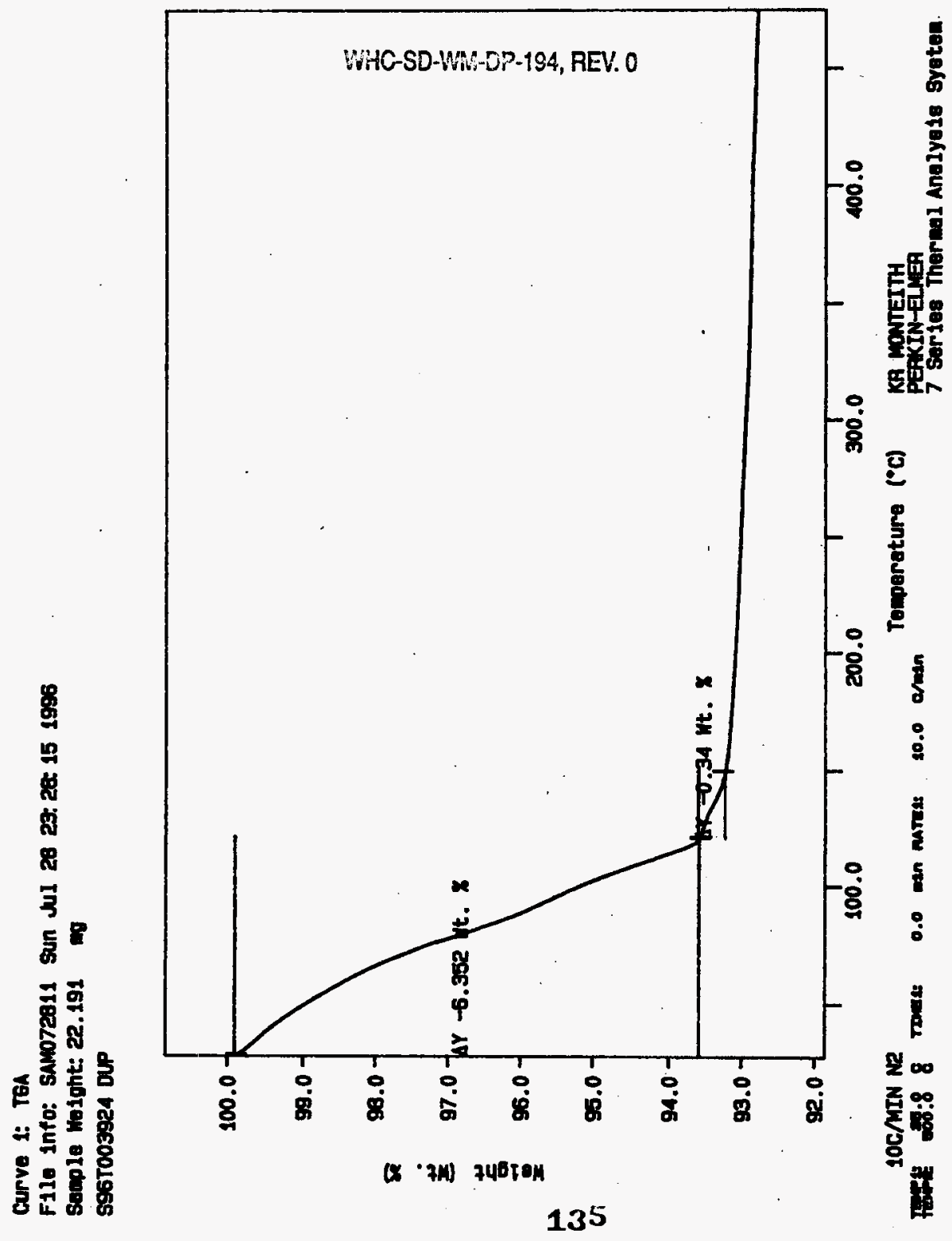


Curve 1; TGA

File Info: SMM072812 Mon Jul 29 01: 38: 421996

Semple Neight: 28.484

S96T003925 SAM

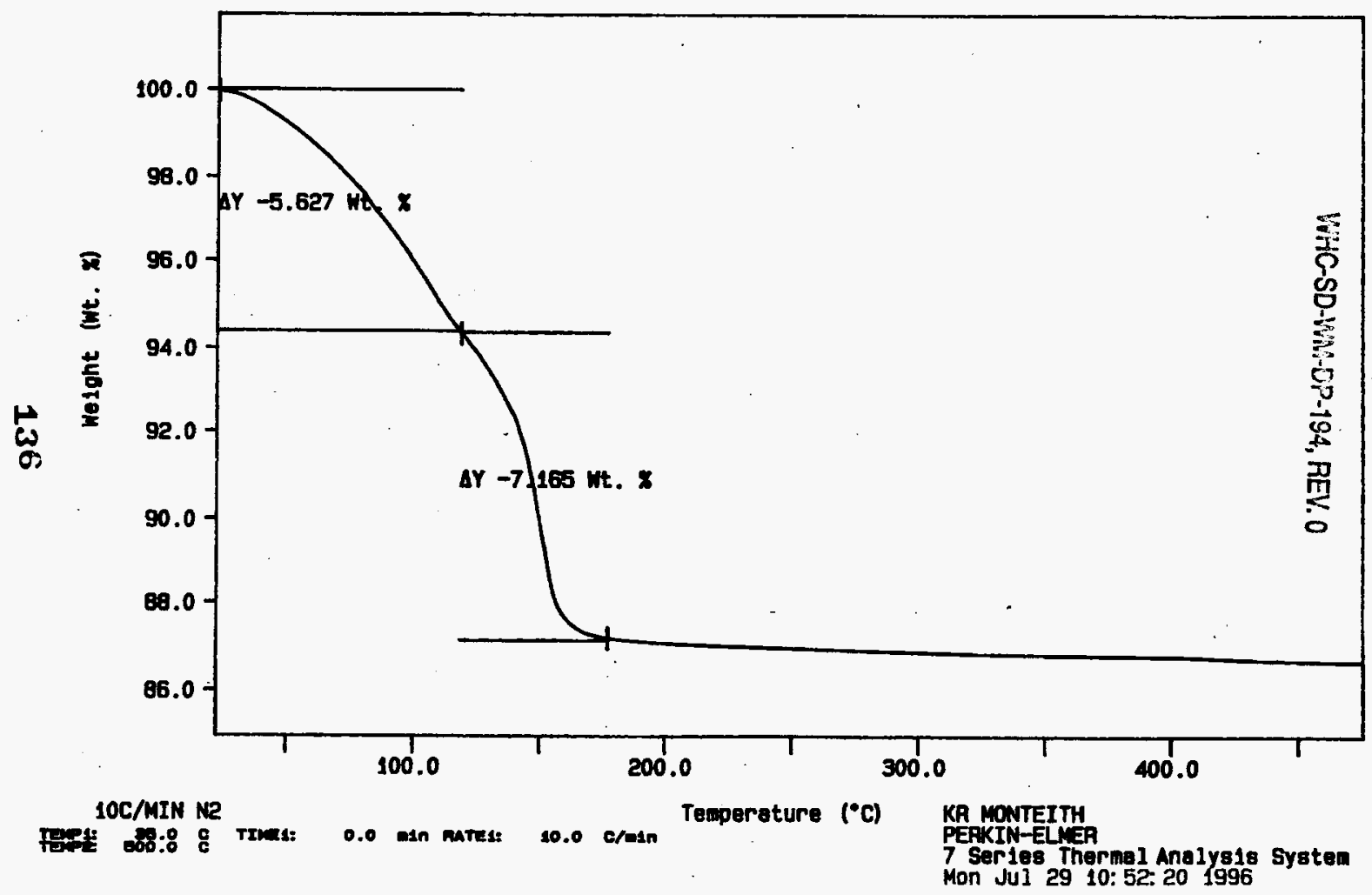




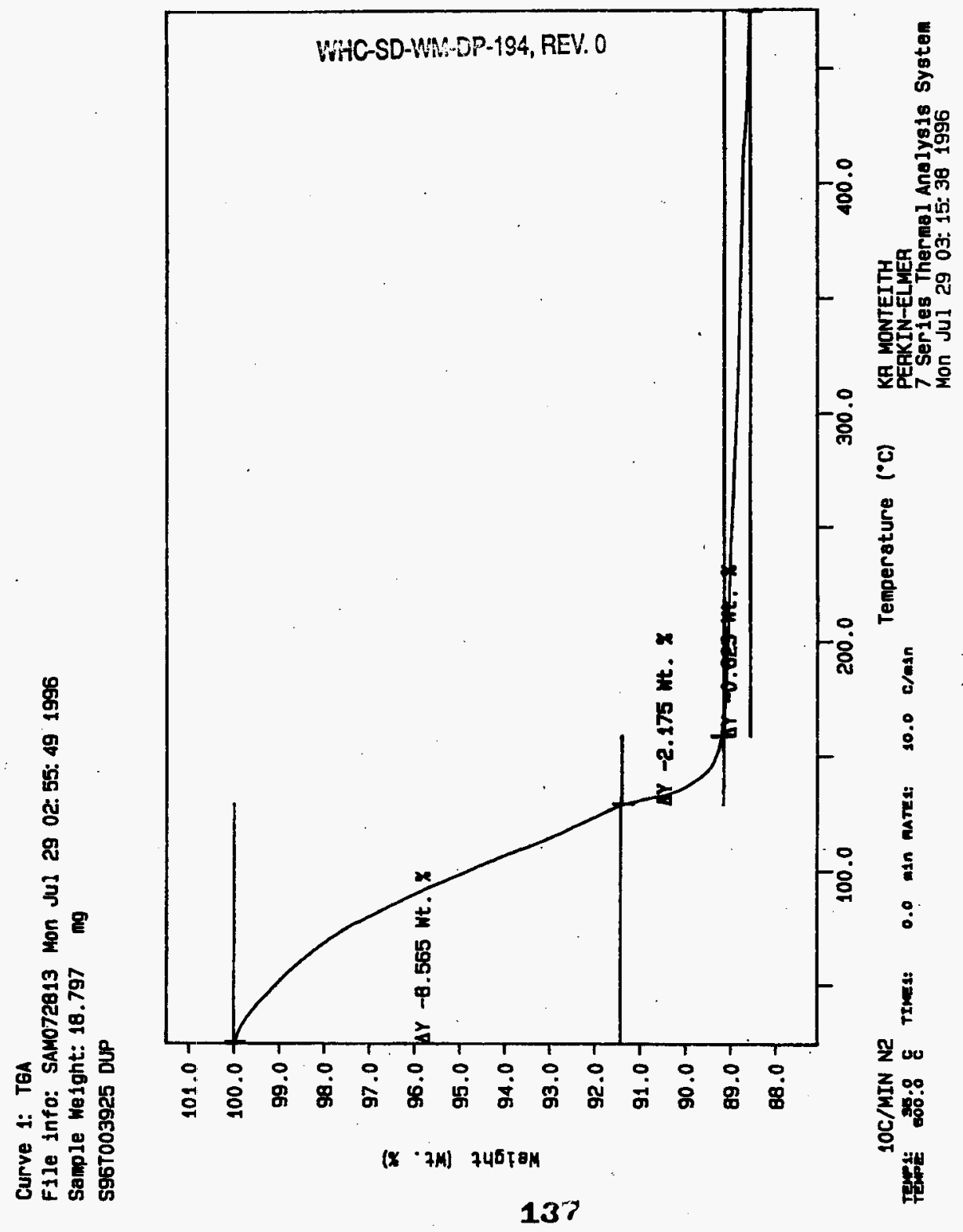




Analyst: $\frac{A D O}{\text { Instrument: TGA0 } 1}$ Book \# $82 \sqrt{8 A}$
Method: LA-560-112 Rev/Mod $B-1$

Worklist Comment: S-109 TGA, RUN UNDER N2. RCJ

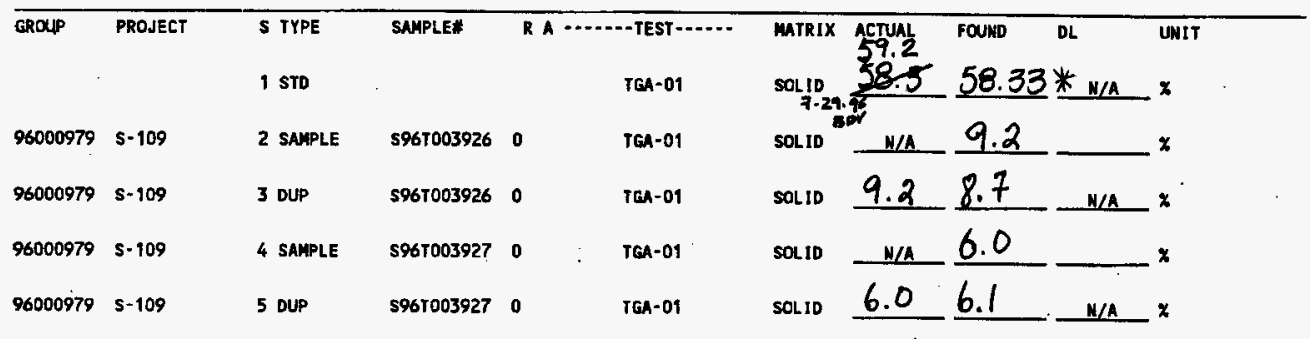

\section{Final page for worklist \# 11168}
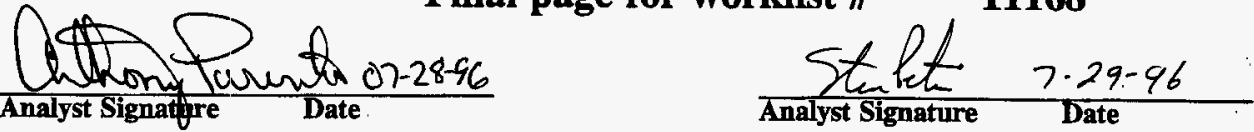

Verified/Validatid by

Bandina

$$
\text { Valenizuela } 8-1-96
$$

\$96T003926 reoults are the Bum of three weight loss steps.

Data Entry Comments:

5967003927 reoults are the sum of two wright loss steps.

Units shown for QC (SPK \& STD) may not reflect the actual units. $D L=$ Detection Limit, $S=$ Worklist Slot Number, $R=$ Replicate Number, $A=$ Aliquot Code. 


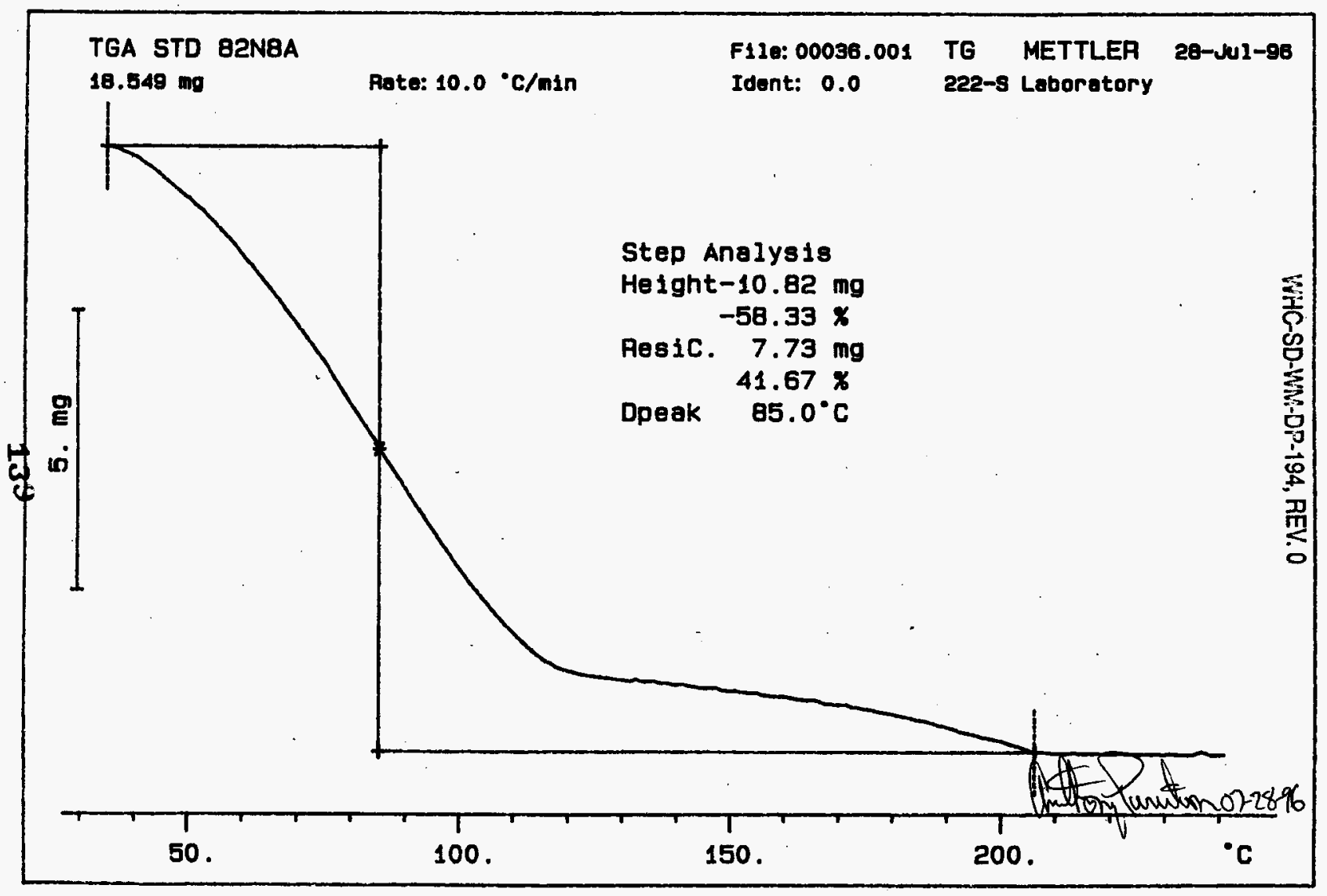




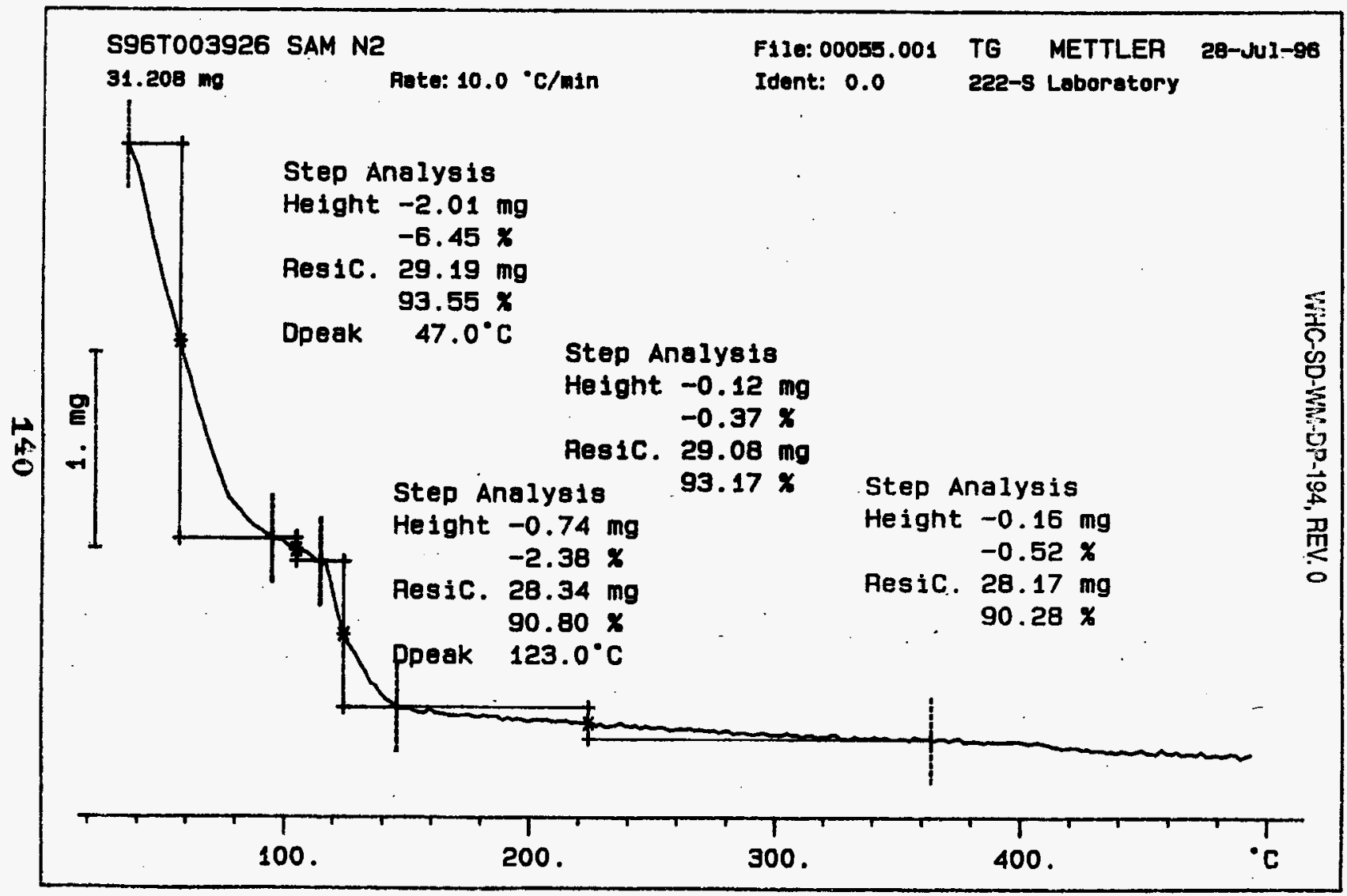




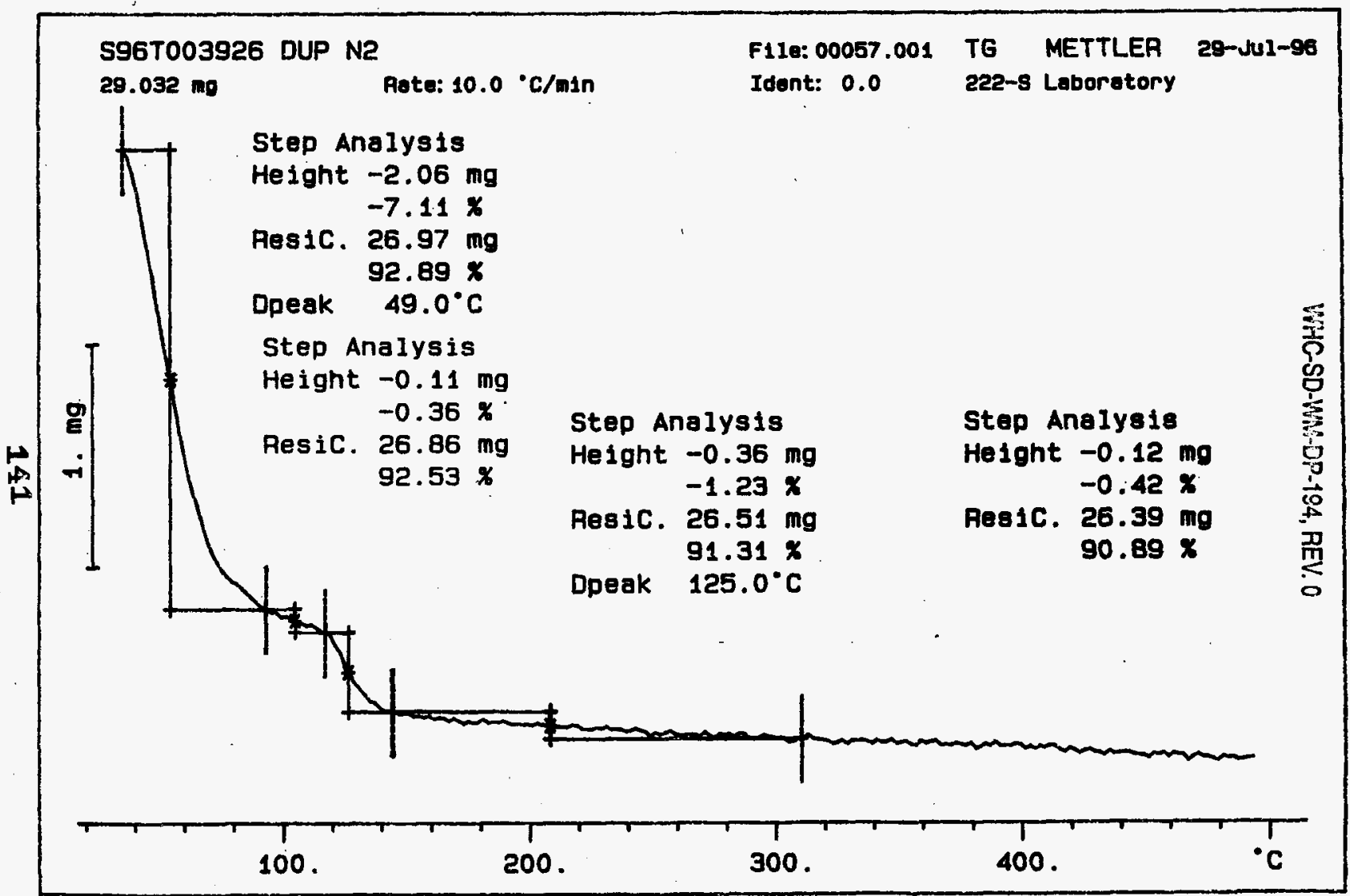




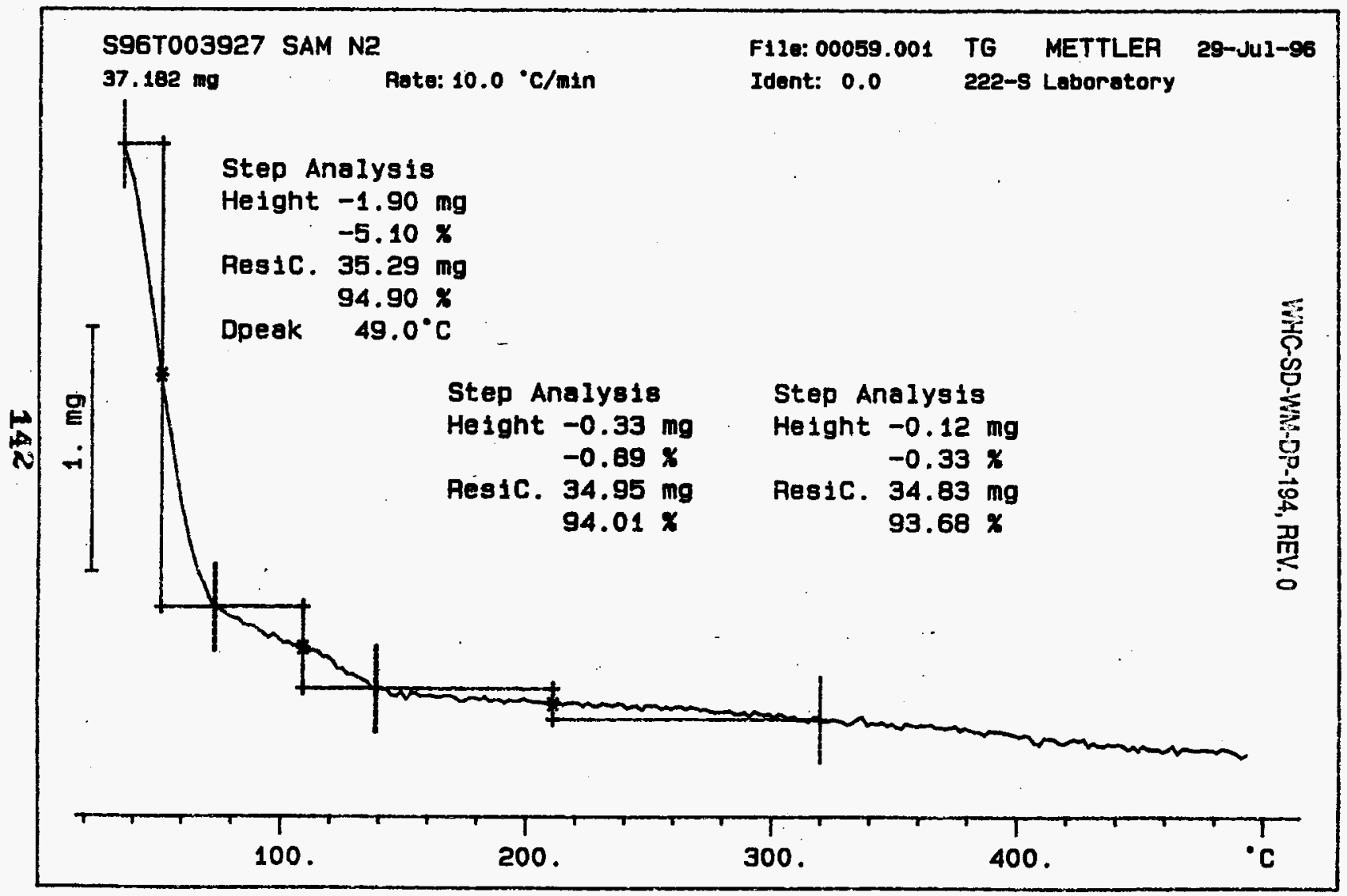




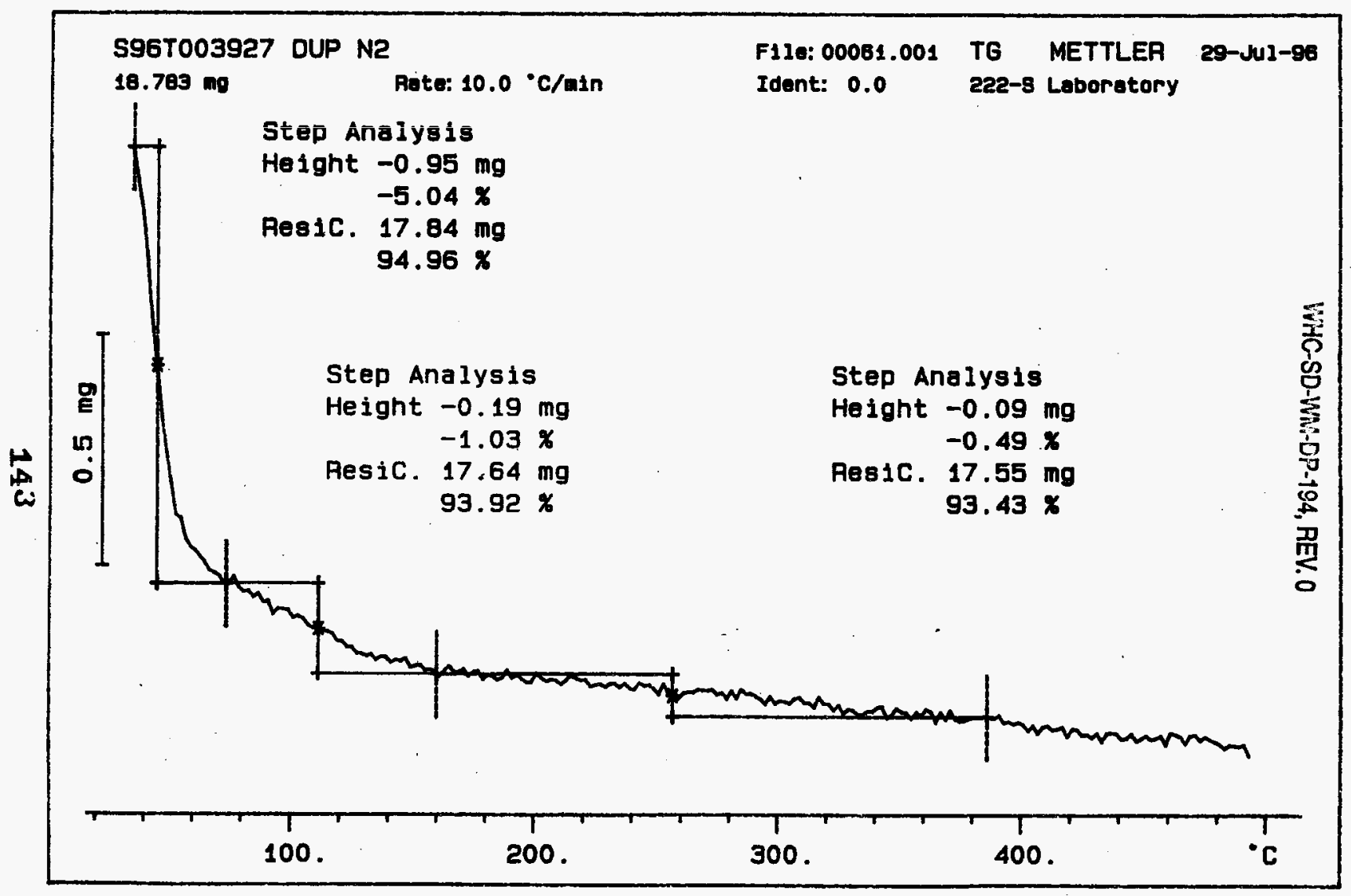


WHC-SD-WM-CP-194, REV. 0

Page: $\quad 1$

worklistrpt Version 2.I 05/15/95

LABCORE Data Entry Template for Worklist\#

11307

Analyst: $\quad$ K'RM Instrument: TGA0 I Book \# Q2N\&A

Method: LA-560-112 Rev/Mod B-1

Worklist Comment: S-109 TGA, RUN UNDER N2. RCJ

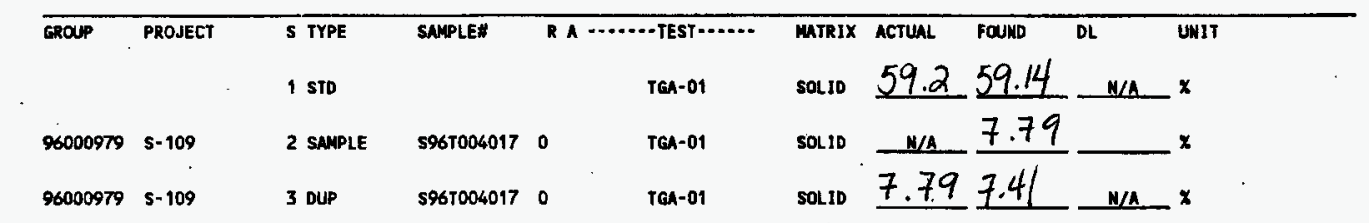
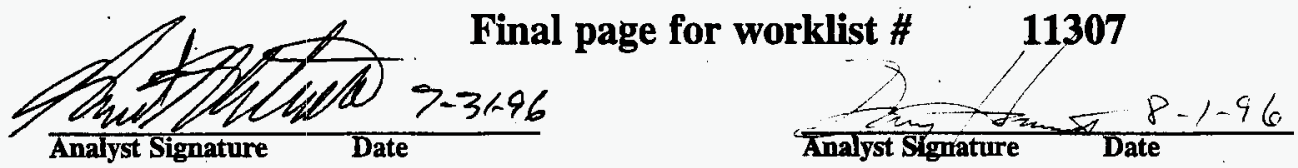

Verified by

Blandina

Valenzuela 8-6.96

Data Entry Cones: the sample contained very little water, therefore the scale is very small showing the vibrations seen by the balance.

Units shown for QC (SPK \& STD) may not reflect the actual units. DL = Detection Limit, $S=$ Worklist Slot Number, $R=$ Replicate Number, $A=$ Aliquot Code.

144 
SIGNATURE BELOW REPRESENTS CHEMICAL TECHNOLOGIST/CHEMIST THAT

COMPLETED/VERIFIED THE CALIBRATION/ANALYSIS ON PAGES 145 TO 147 .

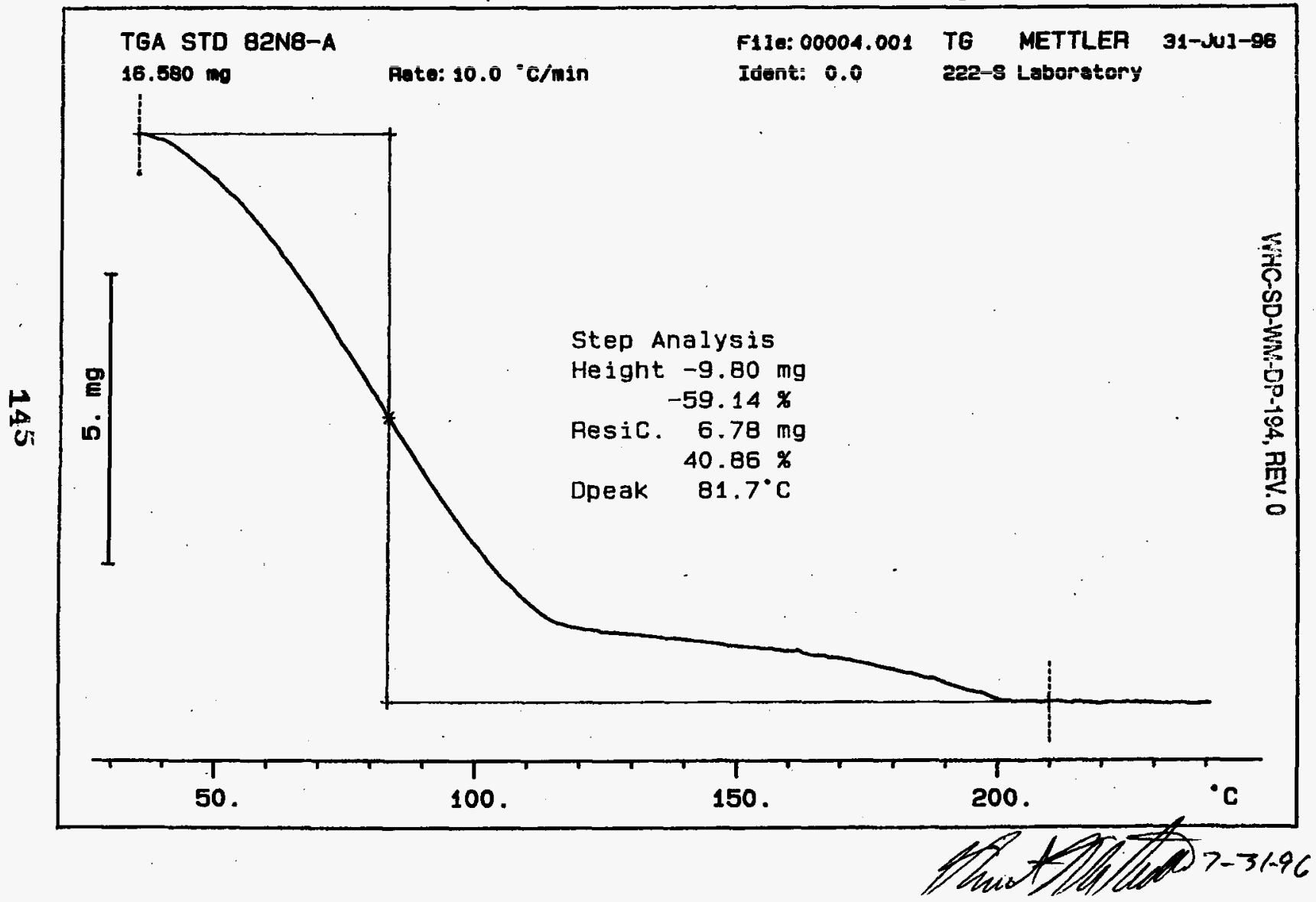




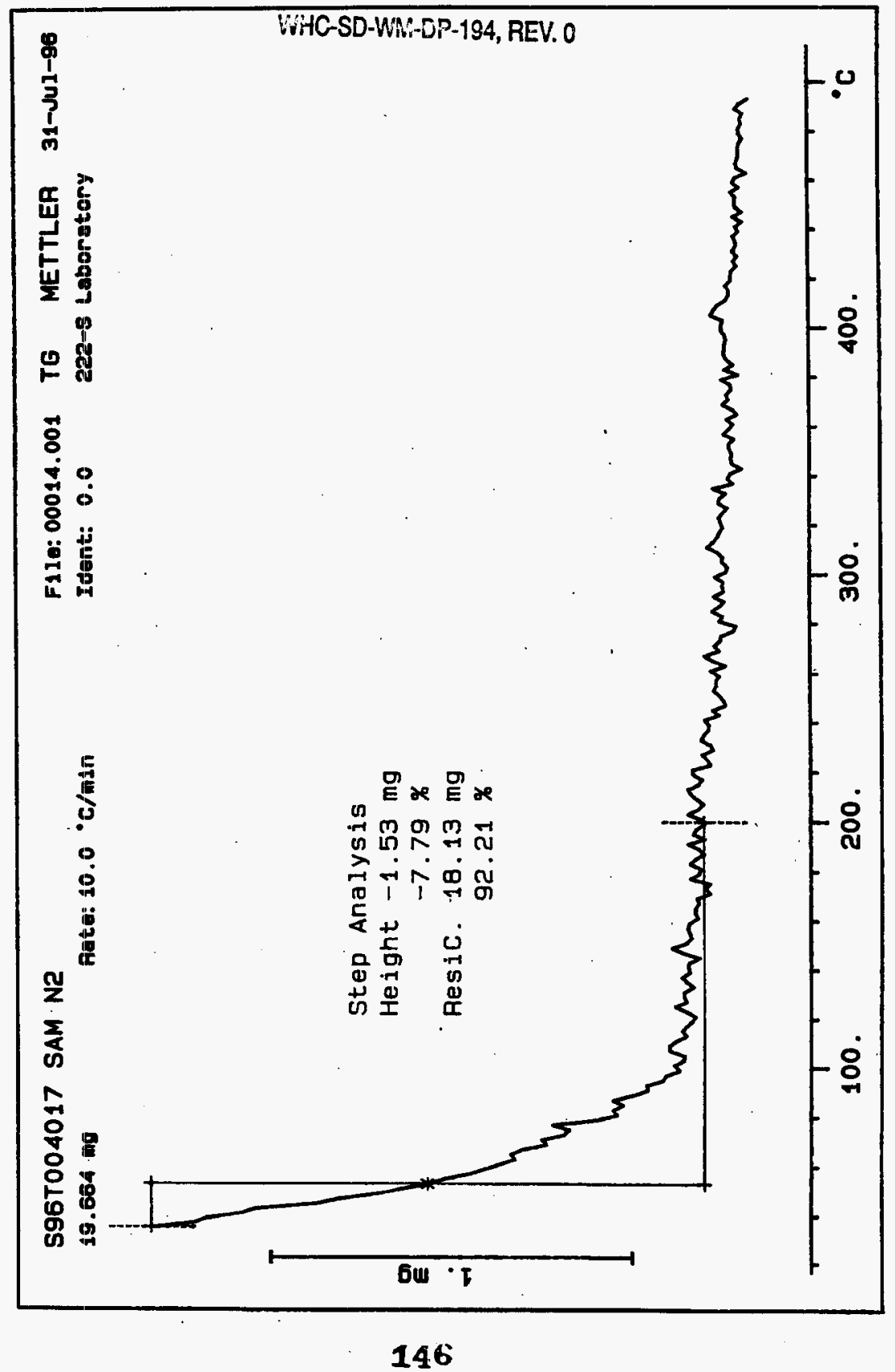




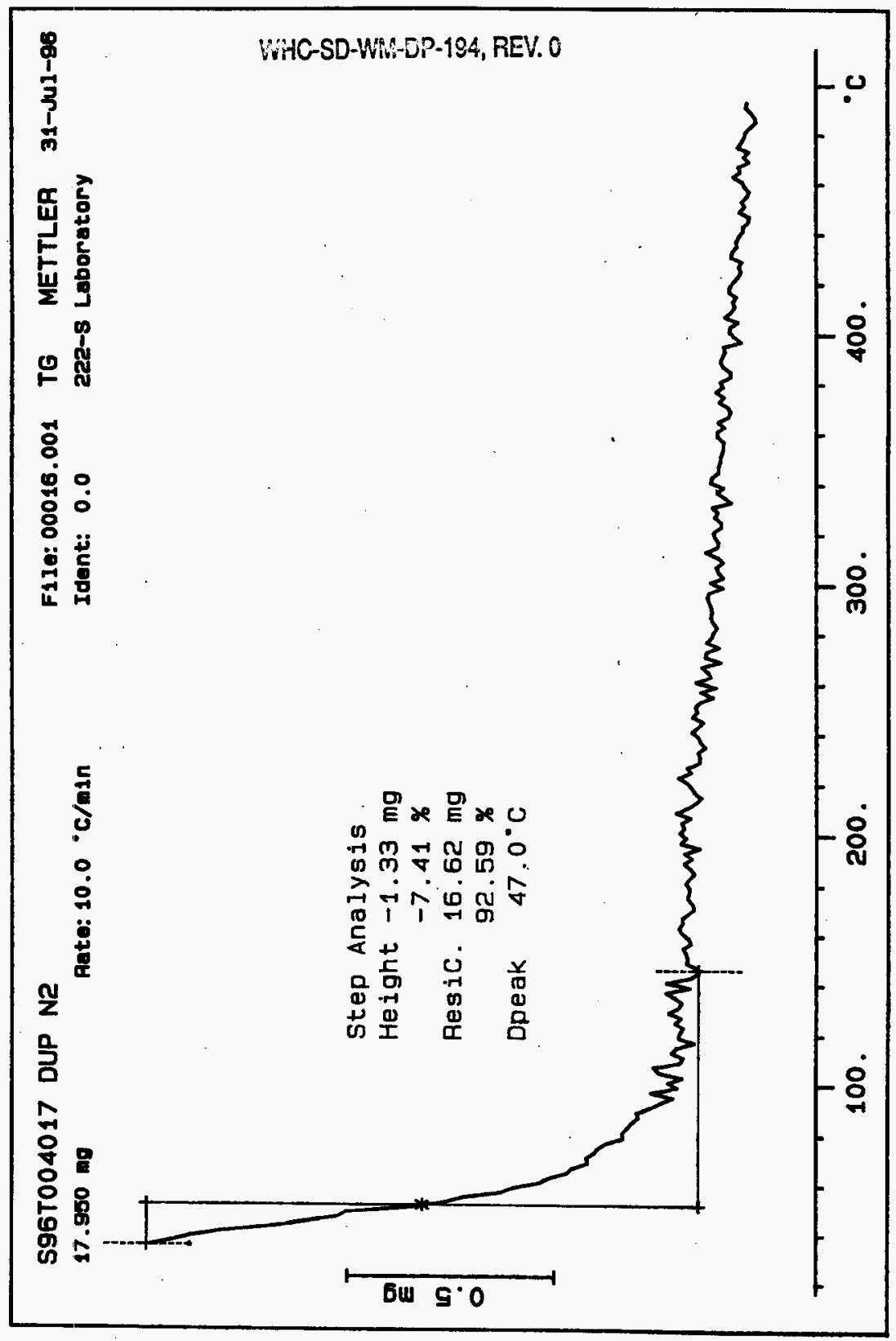

147 


\section{LABCORE Data Entry Template for Worklist\#}

Analyst: $\quad \& A C \quad$ Instrument: TGA0 I Book \# 82N8A

Method: LA-560-112 Rev/Mod B-L

Worklist Comment: S-109 FOR TGA PLEASE RUN UNDER N2

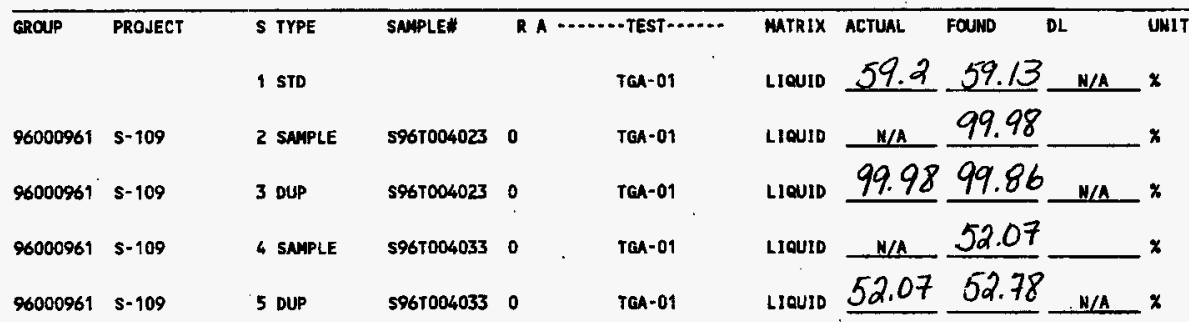

\section{Final page for worklist \#}

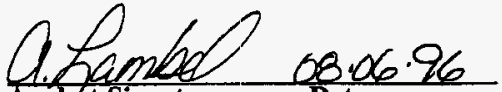

Analyt Signature Date

Verified/Validated by

Blandina

Valenpinelor 8.8 .96

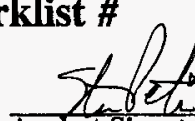

11654
$8-7-96$

Date

Data Entry Conments: S967004033 produced a thermogram that indicates several reactions occuring

Units shown for $Q C$ (SFK \& STD) may not reflect the actual units. $D L=$ Detection Limit, $S=$ Worklist Slot Number, $R=$ Replicate Number, $A=$ Aliquot Code. 


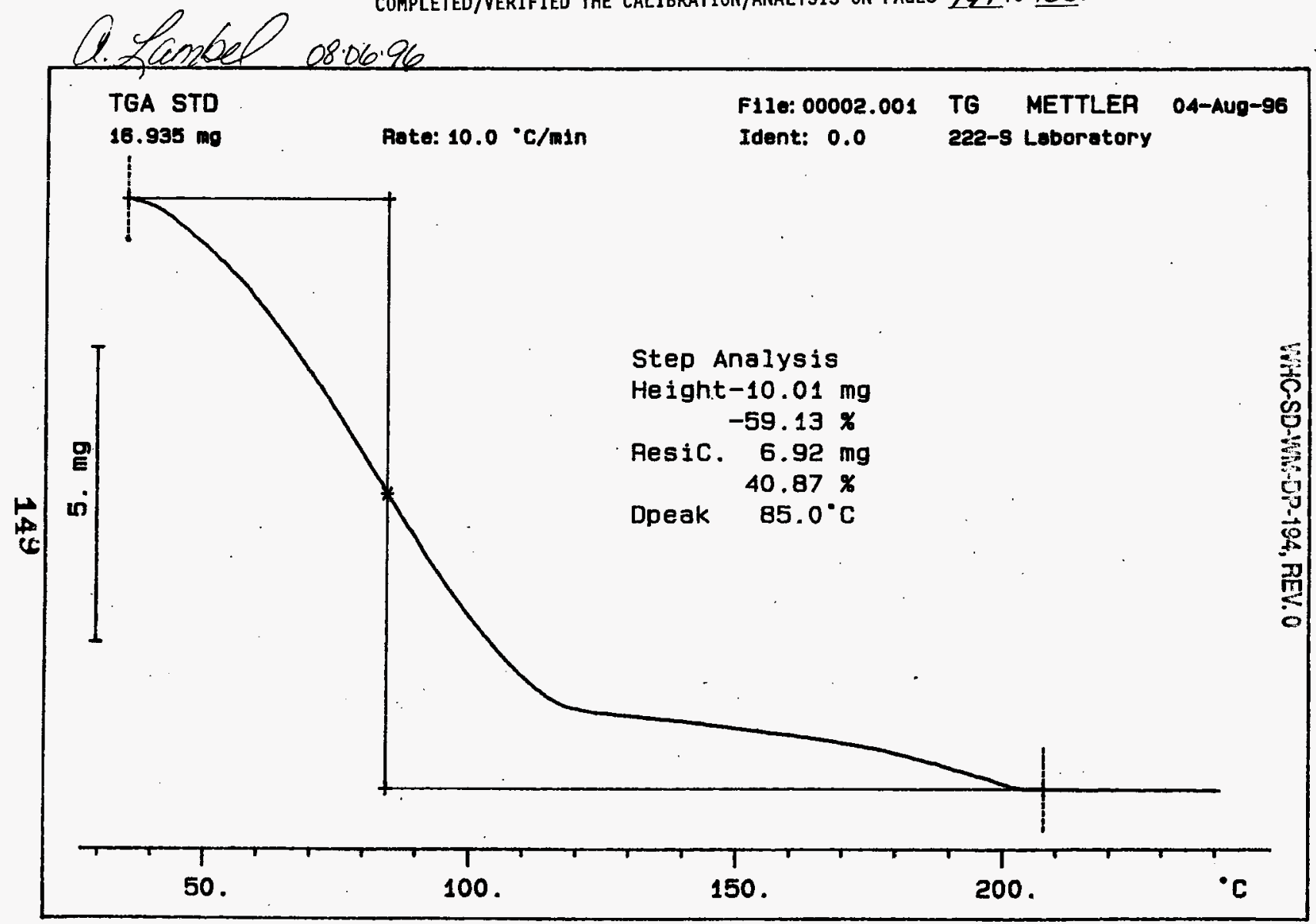




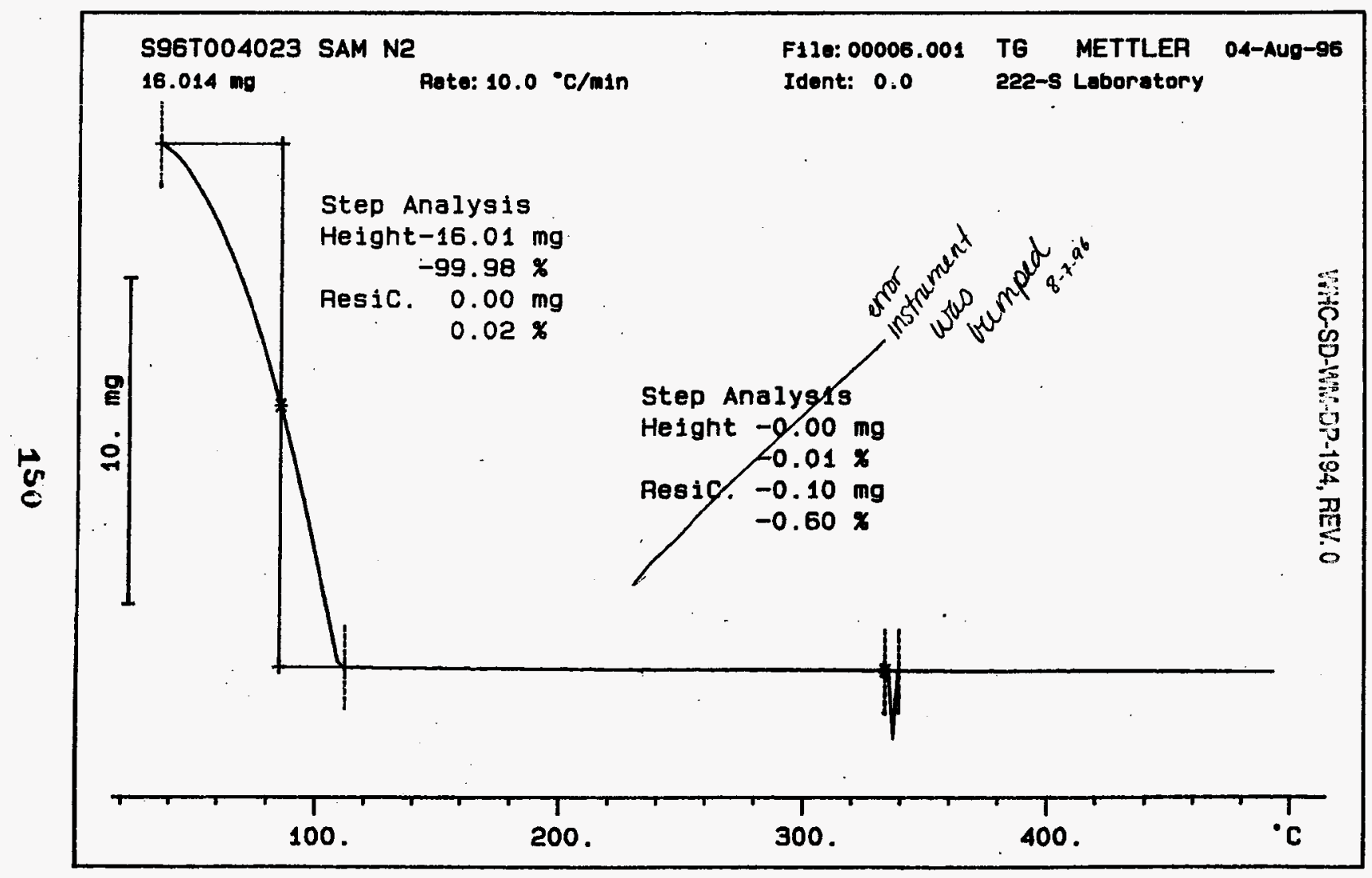




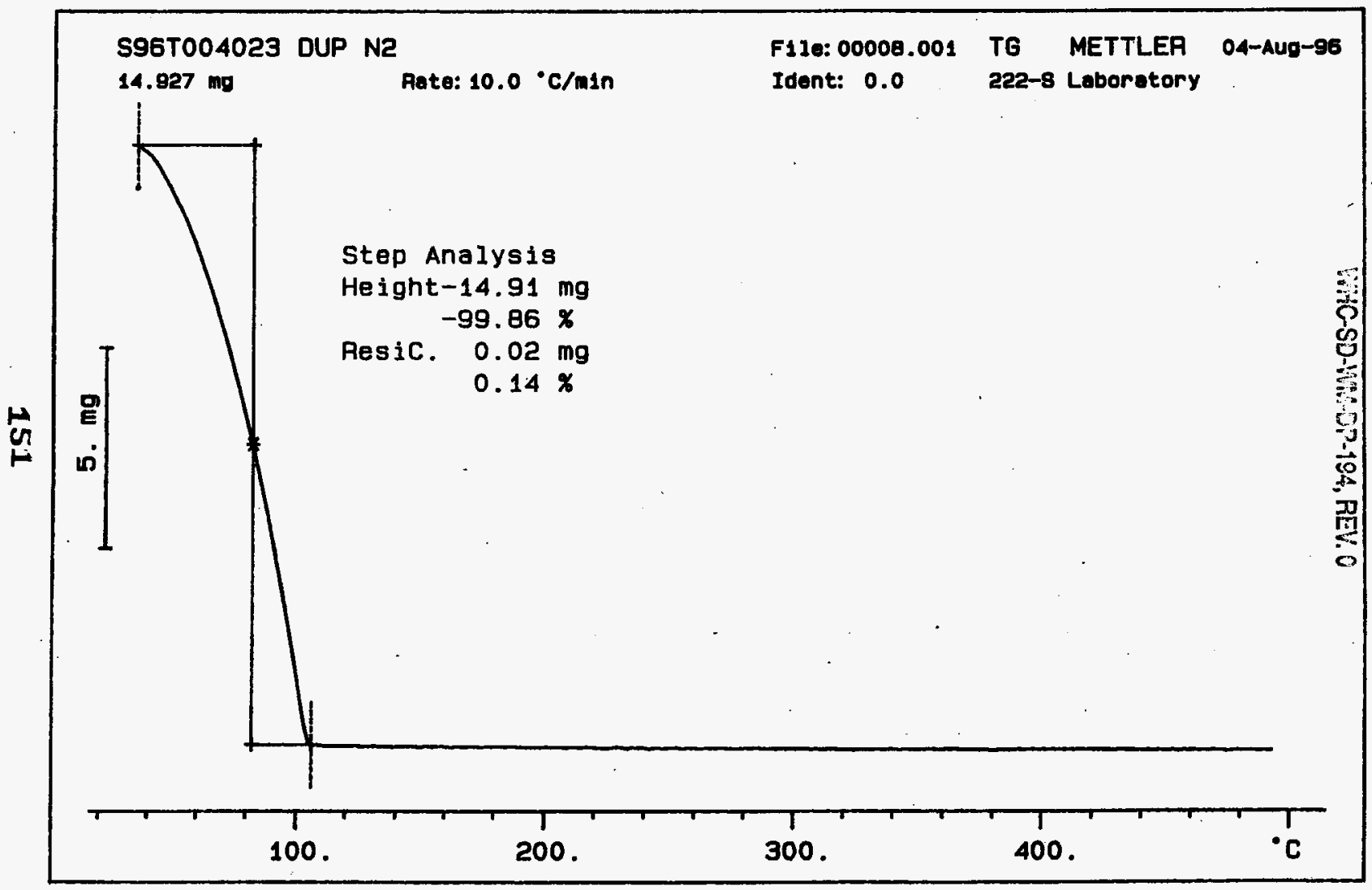




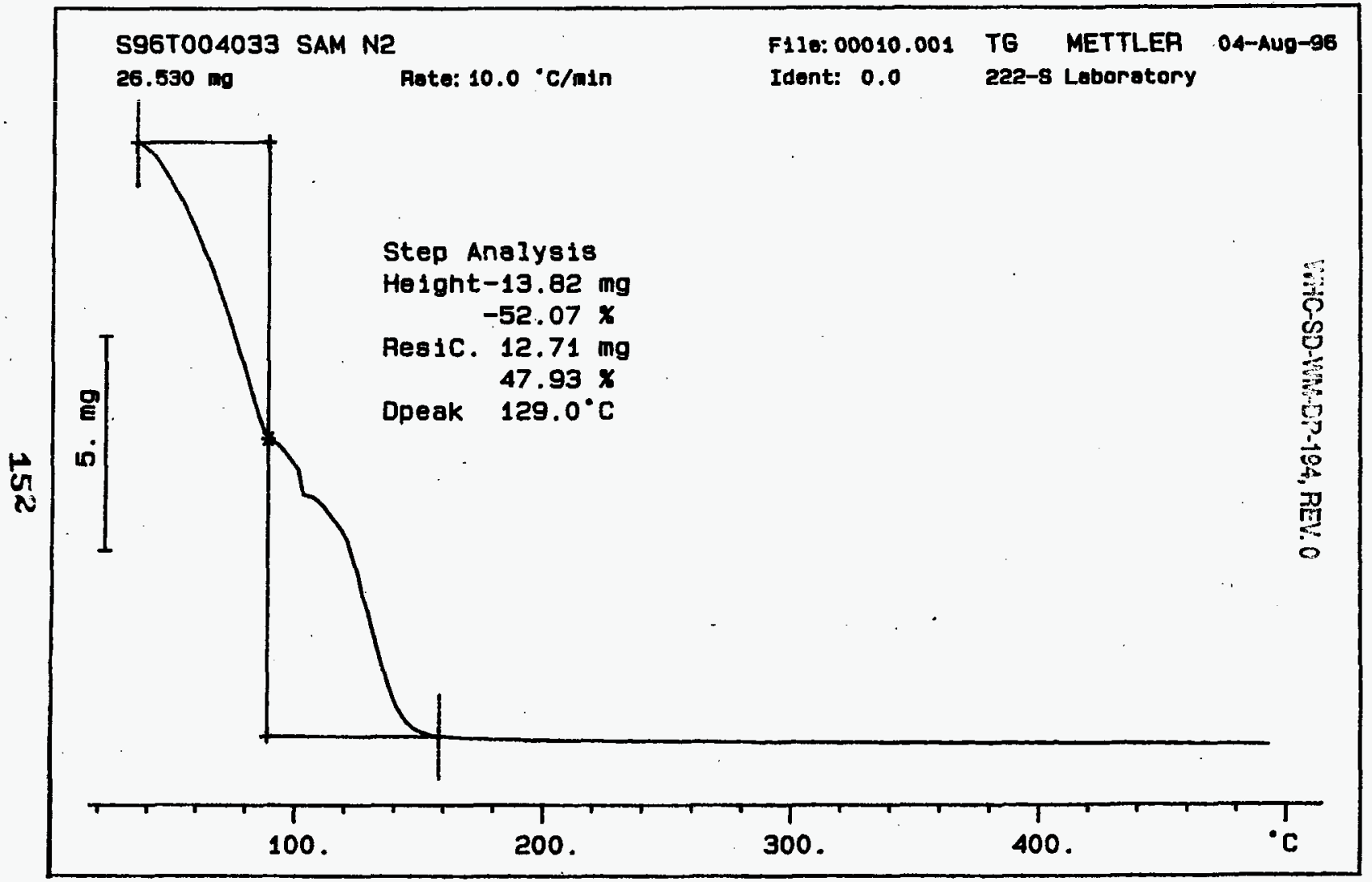




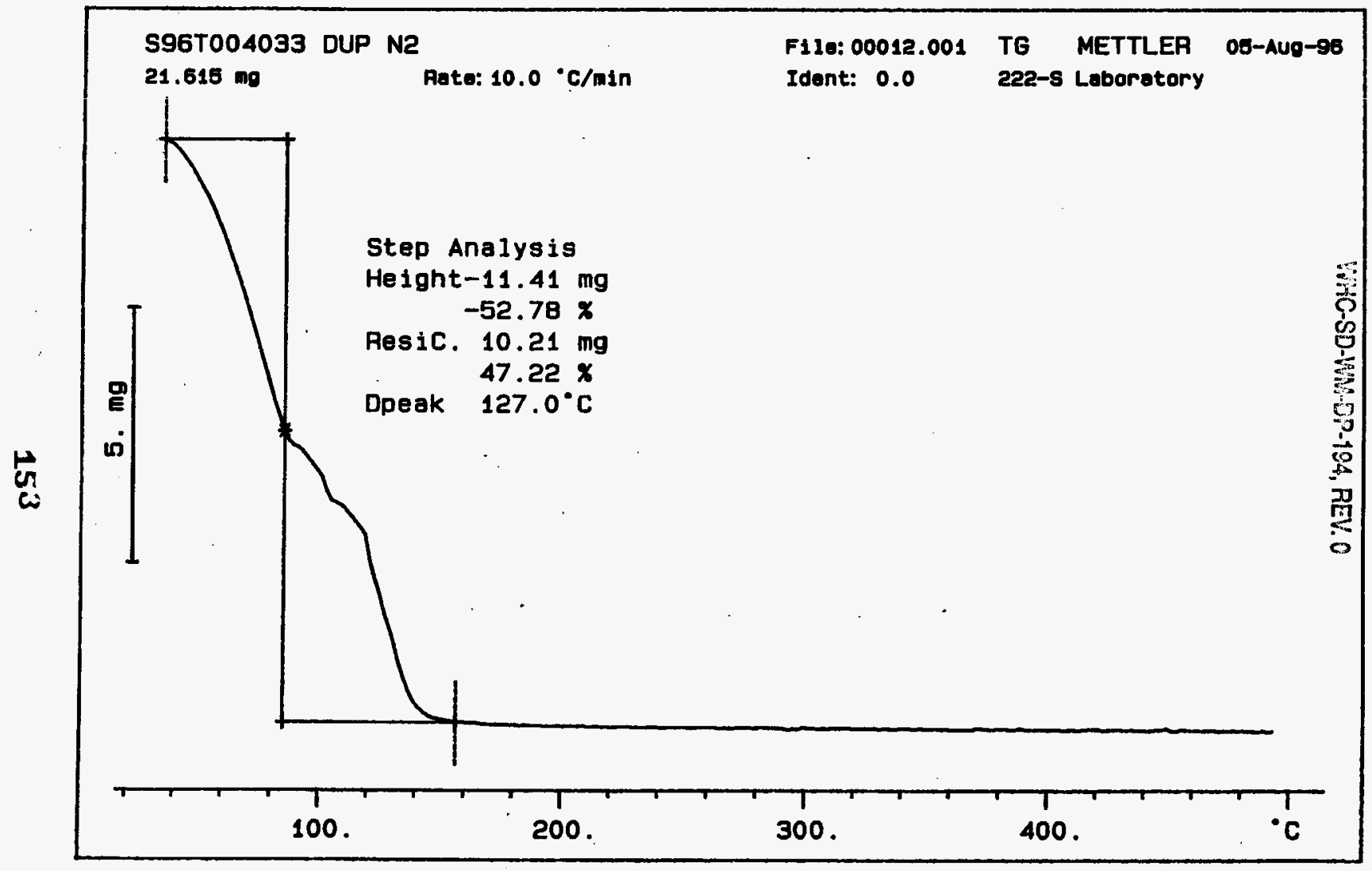


Analyst: $\quad E A L$ Instrument: TGA0 I Book \# 82 N8A

Method: LA-560-11.2 Rev/Mod B-I

Worklist Comment: S-109 FOR TGA PLEASE RUN UNDER N2

\begin{tabular}{|c|c|c|c|c|c|c|c|c|c|c|}
\hline \multirow[t]{2}{*}{ GROUP } & \multirow[t]{2}{*}{ PROJECT } & \multirow{2}{*}{$\begin{array}{l}\text { TYPE } \\
1 \text { STD }\end{array}$} & \multirow[t]{2}{*}{ SAMPLEF } & \multicolumn{2}{|c|}{ 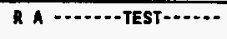 } & \multirow{2}{*}{$\begin{array}{l}\text { MATRIX } \\
\text { SOLID }\end{array}$} & \multirow{2}{*}{$\begin{array}{l}\text { ACTUAL } \\
59.2\end{array}$} & \multirow{2}{*}{$\begin{array}{l}\text { FOUND } \\
59.16 *\end{array}$} & & \multirow[t]{2}{*}{ UNIT } \\
\hline & & & & & TGA-01 & & & & N/A & \\
\hline 96000961 & s-109 & 2 SAMPLE & S967004035 & 0 & TGA-01 & SOLID & $N / A$ & 5.95 & & $x$ \\
\hline \multirow[t]{2}{*}{96000961} & s-109 & 3 Dup & s961004035 & 0 & $\operatorname{Tan}-01$ & SOLID & 5.95 & 6.1 & N/A & $x$ \\
\hline & & 4 STD & & & TGA-01 & SOLTD & 59.2 & 59.33 & $M / A$ & $-x$ \\
\hline 96000961 & $s=109$ & 5 SAMPLE & 5961004036 & 0 & TEA-01 & SOL 1D & N/A & 20.04 & & $x$ \\
\hline 96000961 & s-109 & 6 DUP & S96T004036 & 0 & TEA-01 & SOL1D & 20.04 & 18.57 & N/A & $x$ \\
\hline
\end{tabular}

\section{Final page for worklist \# 11657}

Sie attached lor signatures

Analyst Signature Date $8-7-96$

Verified/Validated by

Blandina

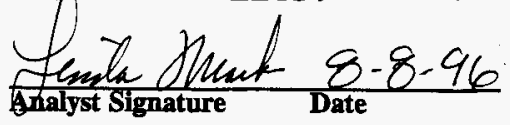

Valenguela 8.8 .96

Data Entry Comments:

Units shown for QC (SPK \& STD) may not reflect the actual units. $D L=$ Detection Limit, $S=$ Worklist Slot Number, $R=$ Replicate Number, $A=$ Aliquot Code. 


\section{LABCORE Data Entry Template for Worklist\#}

Analyst: $\quad$ EAL-1 gmF Instrument: TGAO

Book \#

Method: LA-560-112 Rev/Mod

Worklist Comment: S-109 FOR TGA PLEASE RUN UNDER N2

\begin{tabular}{|c|c|c|c|c|c|c|c|c|c|c|}
\hline \multirow[t]{2}{*}{$\overline{\text { GROUP }}$} & PROJECT & S TYPE & SAMPLE\# & RA & W....-TEST & MTRIX & ACTUAL & FOUND & DL & UNIT \\
\hline & & $1 \mathrm{STD}$ & & & TGA-01 & SOLID & & & $N / A$ & $x$ \\
\hline 96000961 & $5-109$ & 2 SAMPLE & S967004035 & 0 & TGA-01 & SOLID & $H / A$ & & . & $\mathbf{x}$ \\
\hline 96000961 & $s-409$ & 3 DUP & s96ro04035 & 0 & TGA-01 & sol 10 & & & H/A & $\mathbf{x}$ \\
\hline 96000961 & $s-109$ & 4 SAMPLE & 5967004036 & 0 & TGA-01 & SOLID & N/A & & & $x$ \\
\hline 96000961 & $5-109$ & 5 oup & 5967004036 & 0 & TGA-01 & SOLLIO & & & N/A & $\mathbf{x}$ \\
\hline
\end{tabular}

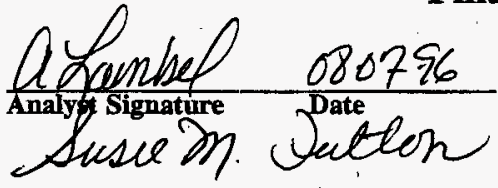

\section{Final page for worklist \# 11657}

\section{Analyst Signature Date}

Data Entry Comments:

Units shown for QC (SPK \& STD) may not reflect the actual wnits. $D L=$ Detection Limit, $S=$ Worklist Slot Number, 


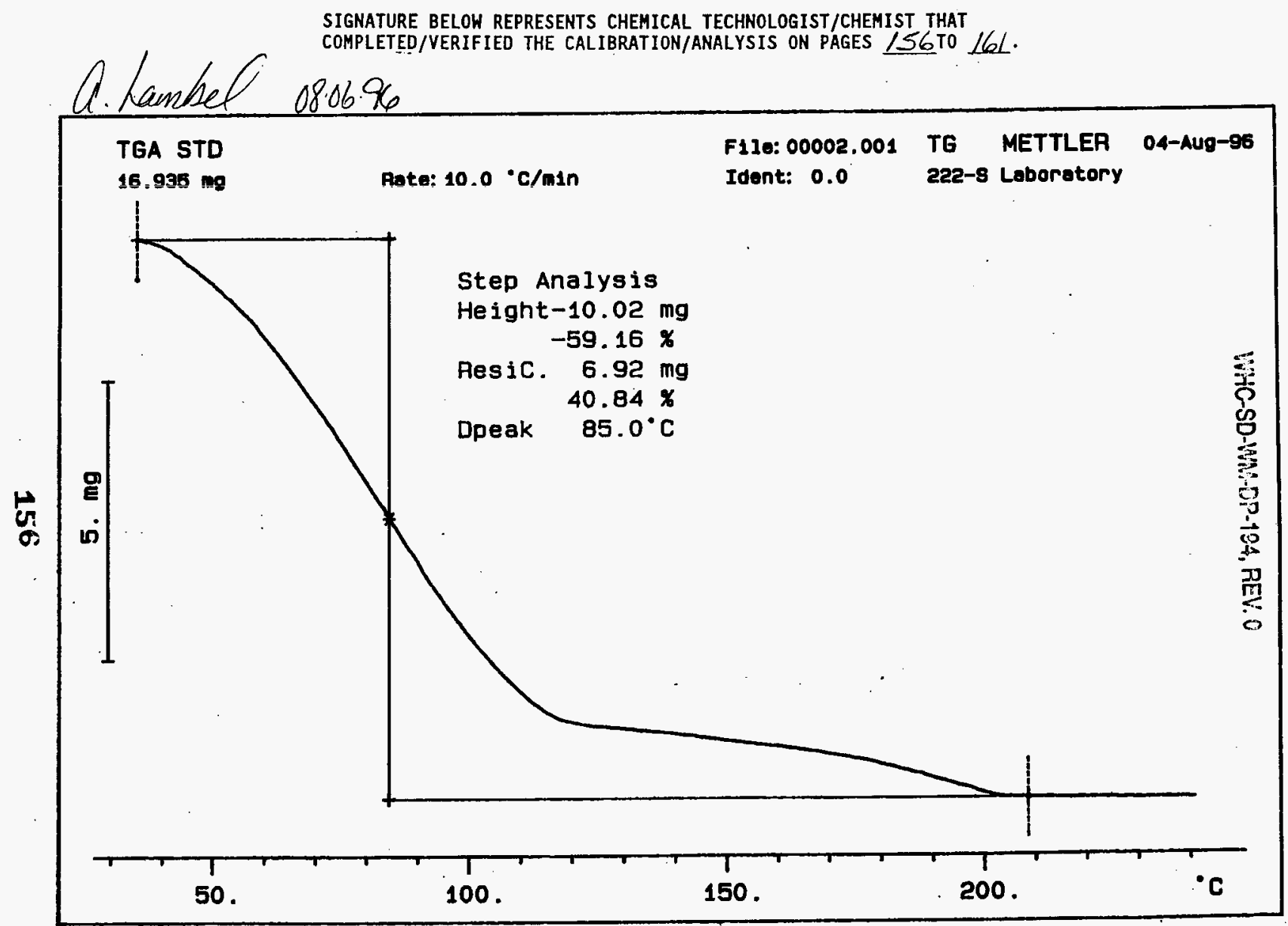




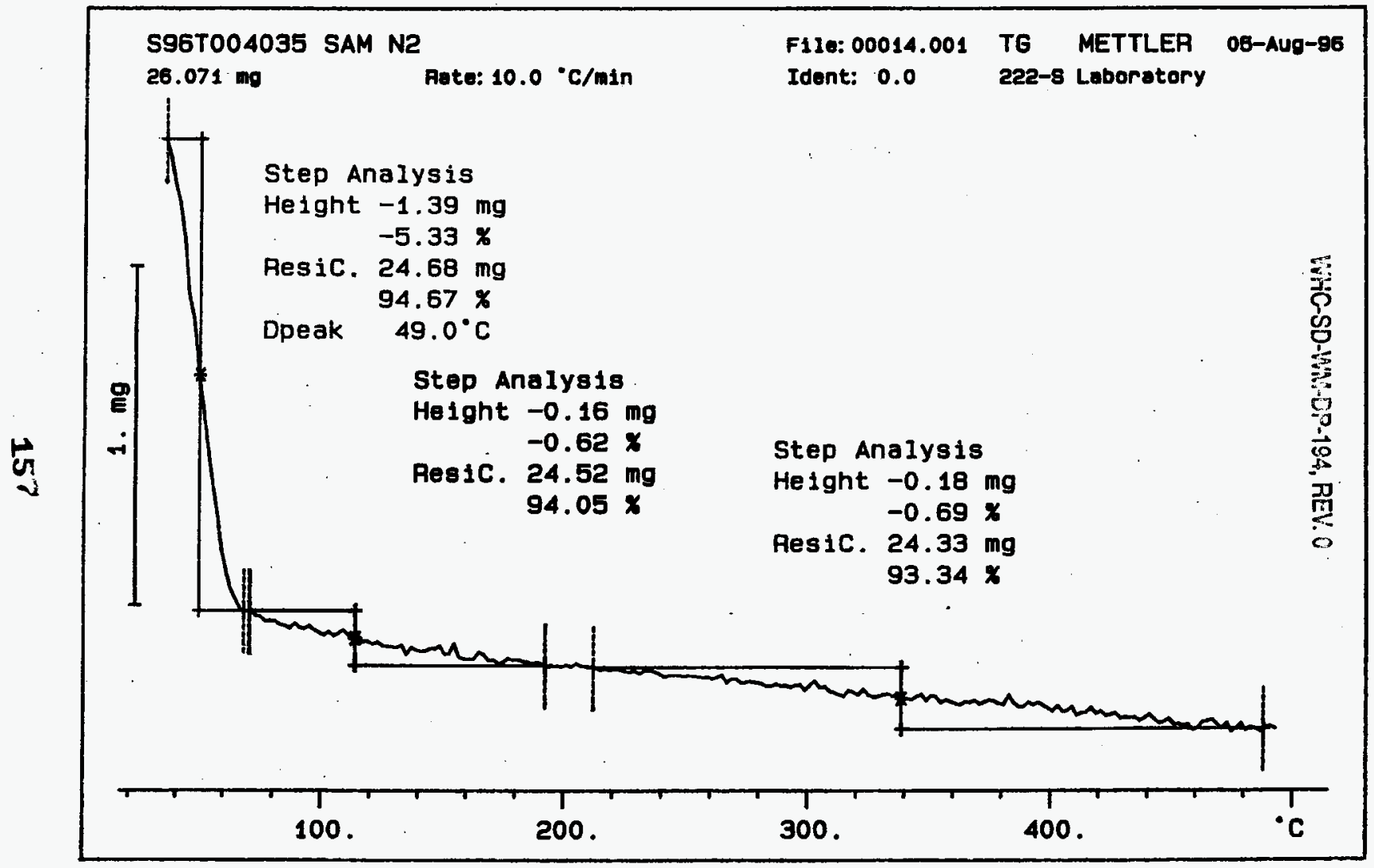




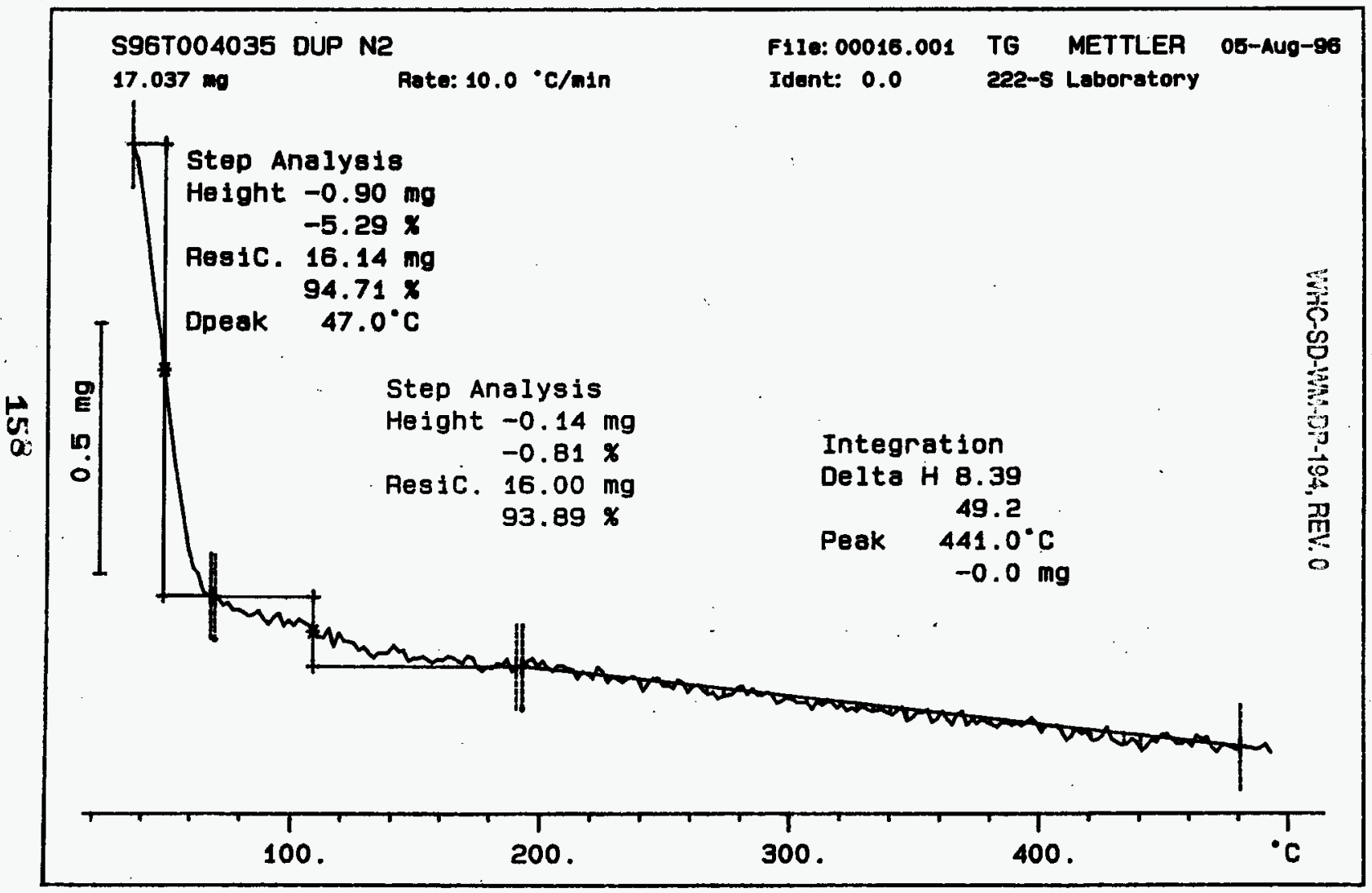




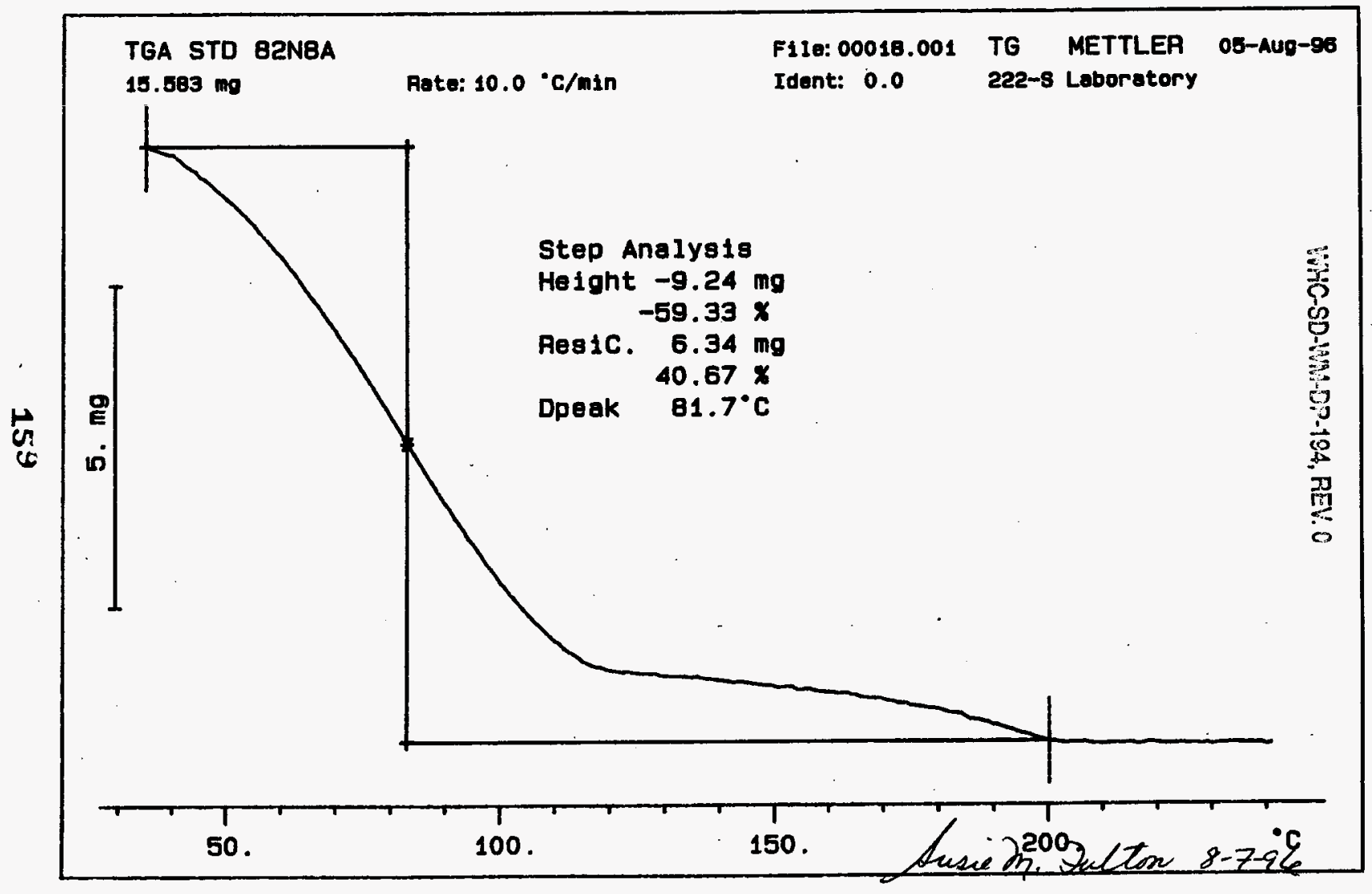




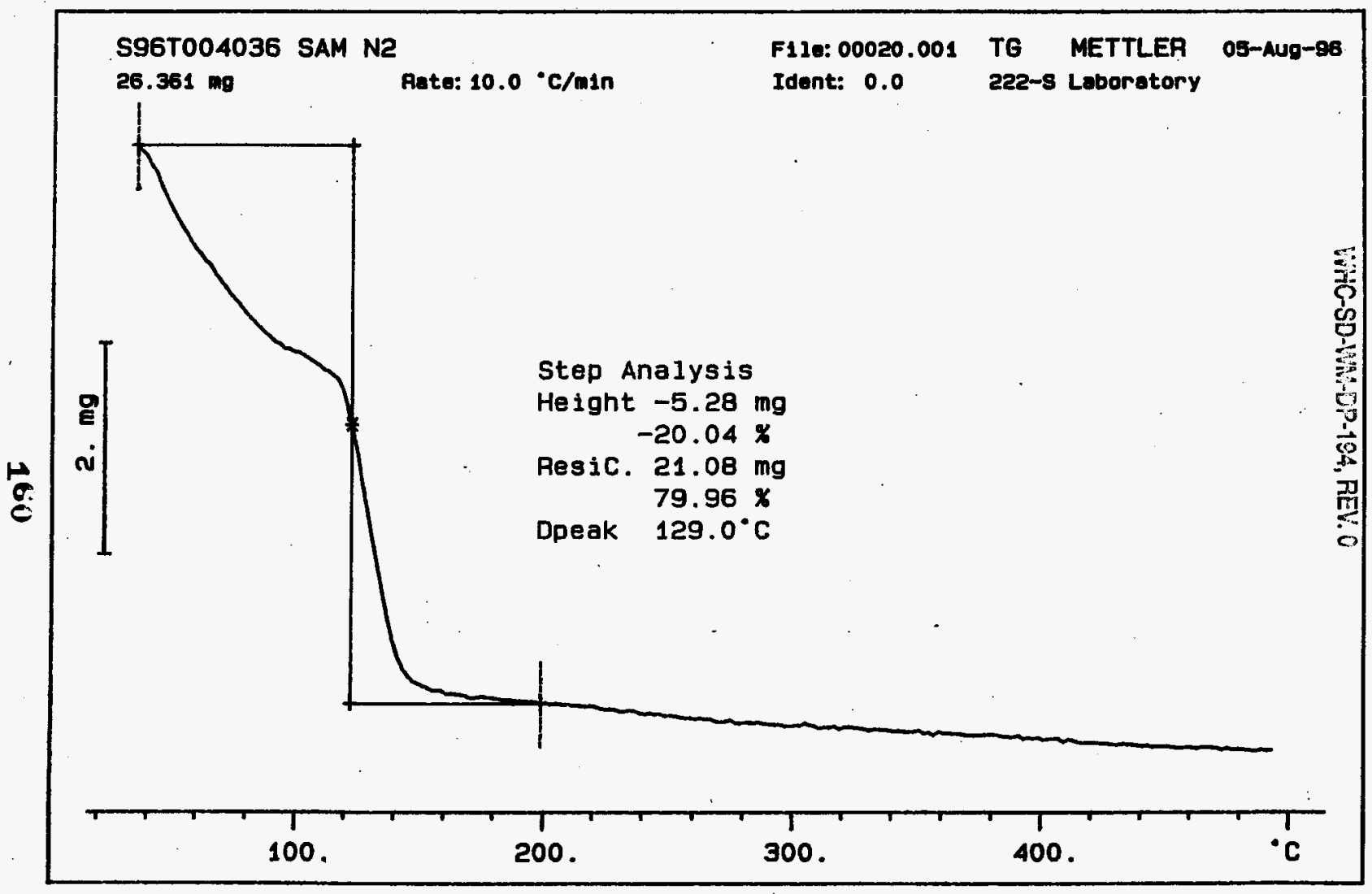




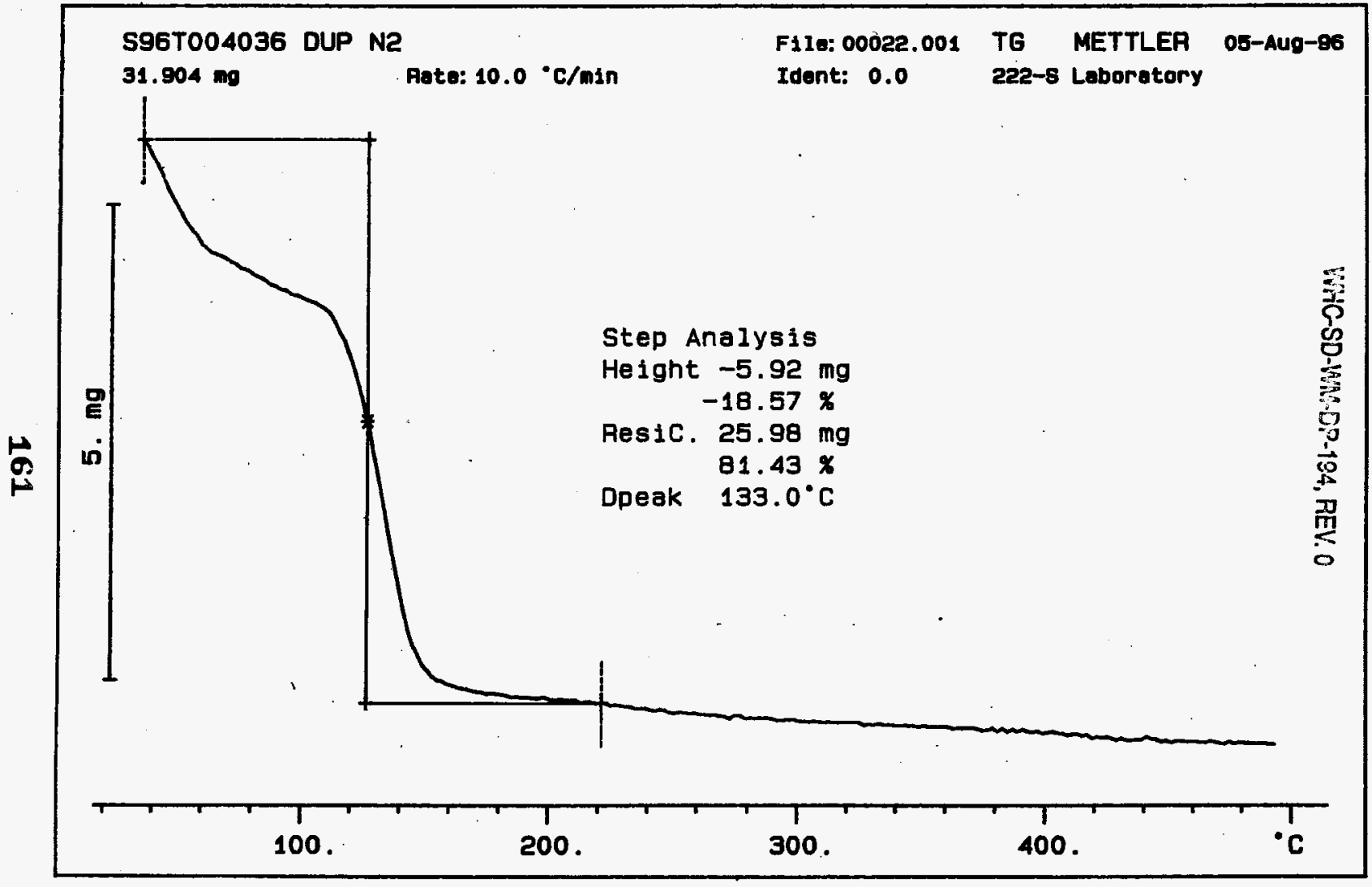




\section{DISTRIBUTION SHEET}

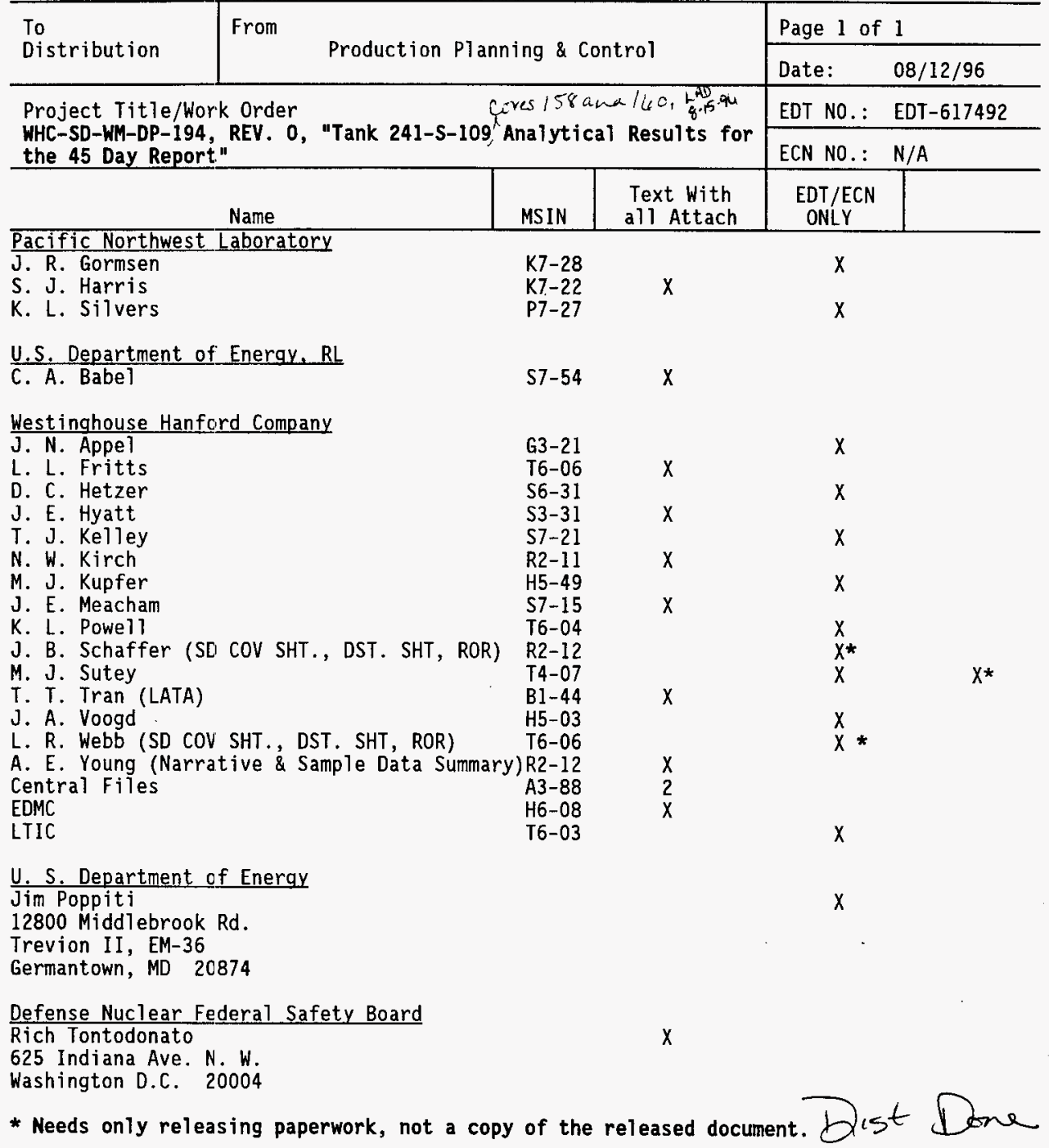

